\title{
Activity-Based Data Fusion for the Automated Progress Tracking of Construction Projects
}

by

\author{
Arash Shahi \\ A thesis \\ presented to the University of Waterloo \\ in fulfillment of the \\ thesis requirement for the degree of \\ Doctor of Philosophy \\ in
}

Civil Engineering

Waterloo, Ontario, Canada, 2012

(CArash Shahi 2012 


\section{AUTHOR'S DECLARATION}

I hereby declare that I am the sole author of this thesis. This is a true copy of the thesis, including any required final revisions, as accepted by my examiners.

I understand that my thesis may be made electronically available to the public. 


\begin{abstract}
In recent years, many researchers have investigated automated progress tracking for construction projects. These efforts range from 2D photo-feature extraction to $3 \mathrm{D}$ laser scanners and radio frequency identification (RFID) tags. A multi-sensor data fusion model that utilizes multiple sources of information would provide a better alternative than a singlesource model for tracking project progress. However, many existing fusion models are based on data fusion at the sensor and object levels and are therefore incapable of capturing critical information regarding a number of activities and processes on a construction site, particularly those related to non-structural trades such as welding, inspection, and installation activities.

In this research, a workflow based data fusion framework is developed for construction progress, quality and productivity assessment. The developed model is based on tracking construction activities as well as objects, in contrast to the existing sensor-based models that are focussed on tracking objects. Data sources include high frequency automated technologies including 3D imaging and ultra-wide band (UWB) positioning. Foreman reports, schedule information, and other data sources are included as well. Data fusion and management process workflow implementation via a distributed computing network and archiving using a cloud-based architecture are both illustrated. Validation was achieved using a detailed laboratory experimental program as well as an extensive field implementation project. The field implementation was conducted using five months of data acquired on the University of Waterloo Engineering VI construction project, yielding promising results. The data fusion processes of this research provide more accurate and more reliable progress and earned value estimates for construction project activities, while the developed data management processes enable the secure sharing and management of construction research data with the construction industry stakeholders as well as with researchers from other institutions.
\end{abstract}




\section{Acknowledgements}

I would like to express my most sincere gratitude and appreciation to my supervisors, Prof. Carl T. Haas and Prof. Jeffrey S. West, for their mentorship, guidance, encouragement, and exceptional support throughout my many years at the University of Waterloo. I was also privileged to have the support of Prof. Ralph Haas, Prof. Keith Hipel, and Prof. Tarek Hegazi, both as members of a great Ph.D. committee and wonderful mentors, from whom I have learned such valuable lessons. I would also like to thank Prof. Brenda McCabe for agreeing to be the external examiner for my PhD defence.

I would also like to acknowledge and appreciate the collaboration and helpful advice of Dr. Mahmoud Ahmed as well as the invaluable assistance of Jose Cardona and fellow graduate students Afrooz Aryan, Yelda Turkan, Adrien Guillemet, A. J. Antony Chettupuzha, Hasaan Nasir, and Samin Shokri. It was also a pleasure to work with our undergraduate assistants, Rida Abdullah and Kaitlin Carlson, on a number of aspects of this research.

The support from Gary Caldwall of Aecon Buildings in granting full access to his site at the University of Waterloo and exceptional help during the data collection and the analysis phase of my work is very much appreciated. I would also like to recognize the support and collaborative efforts of Ray Simonson, Sandra MacGillivray, Erik Vander Ahe and Jordan Chelsom of COREWORX Inc. in developing the research database presented in this thesis. The support from NSERC-CGS for funding this research is also acknowledged and appreciated.

I am particularly grateful to my parents Sussan and Davood and to my sister Sheida for their unwavering support and continual encouragement. Special thanks to all my friends, especially Saeed, Ehsan, and Saman, and to my cousin and mentor Mahdi Safa and his wife Maryam Vasefi, all for their unconditional support and love, and most importantly, for being the family I very much needed in Canada. 


\section{Dedication}

To my mother, Sussan 


\section{Table of Contents}

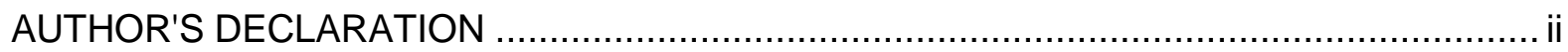

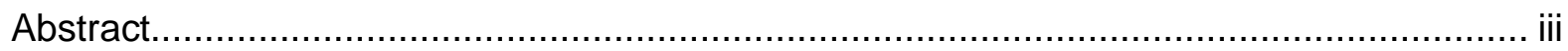

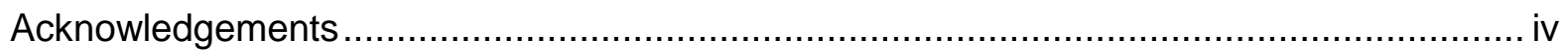

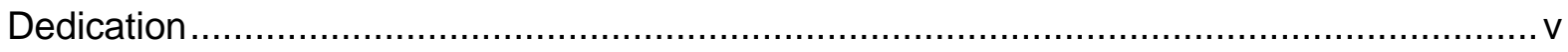

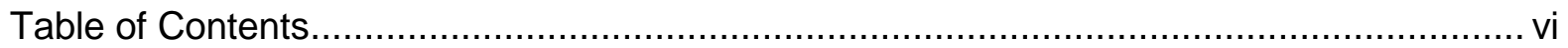

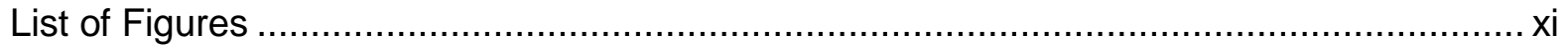

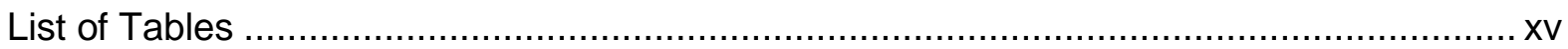

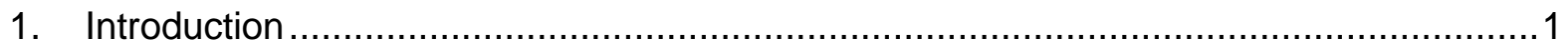

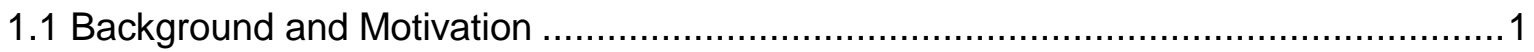

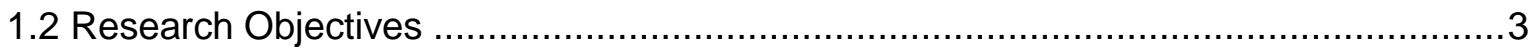

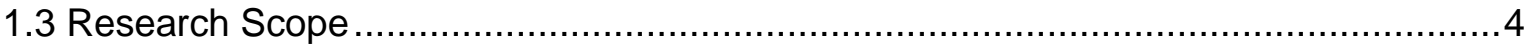

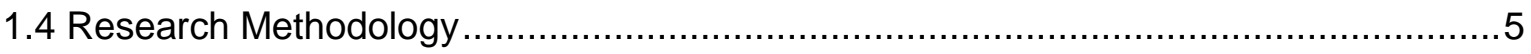

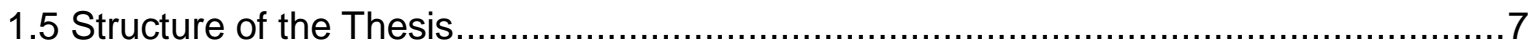

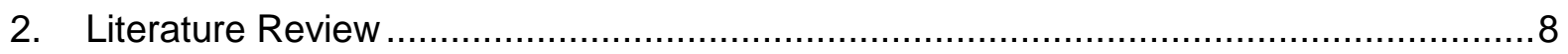

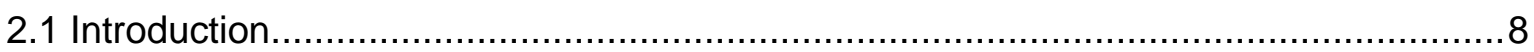

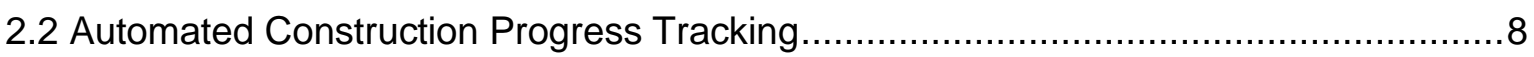

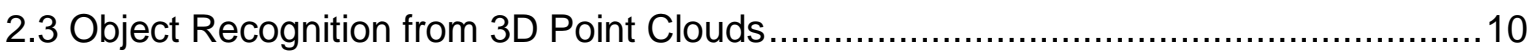

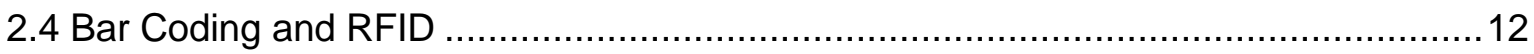

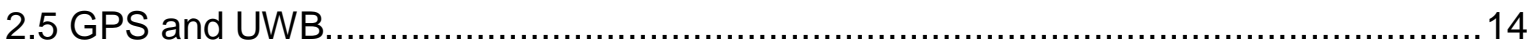

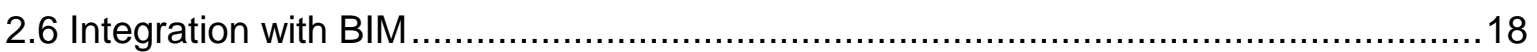

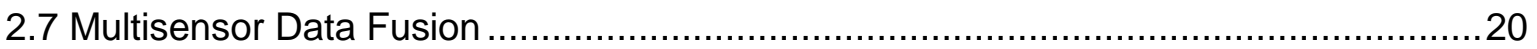




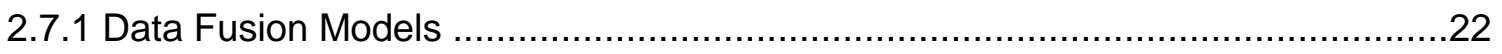

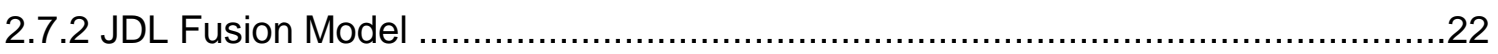

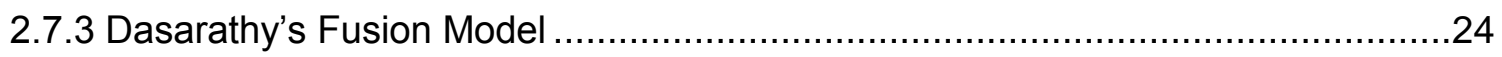

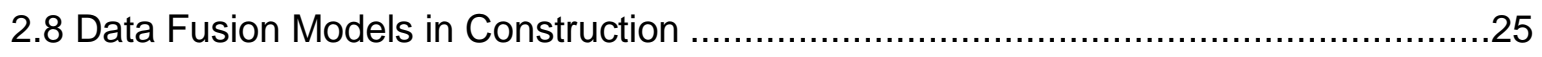

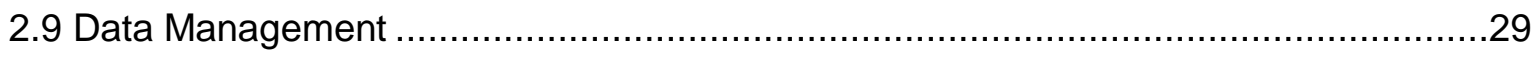

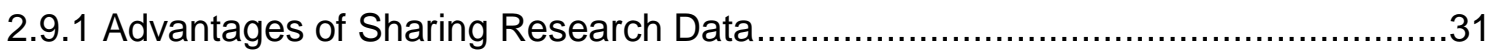

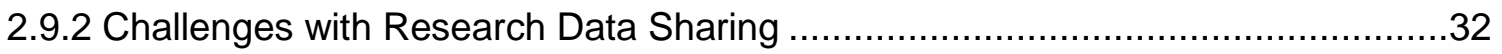

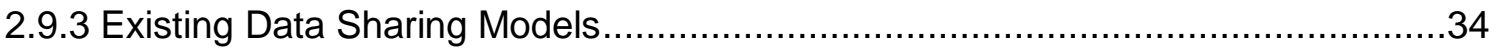

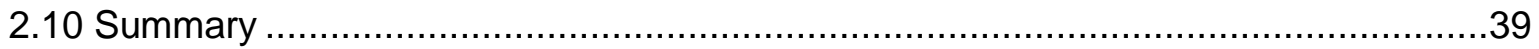

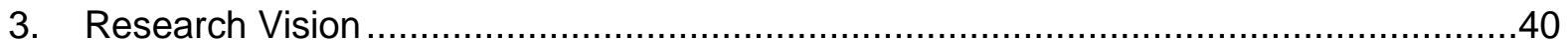

3.1 Knowledge Gaps from Literature Review ………….............................................40

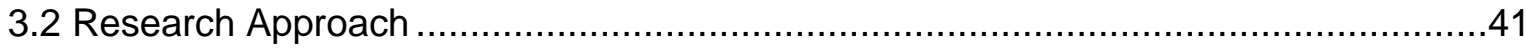

3.2.1 Workflow Driven Design ................................................................................ 41

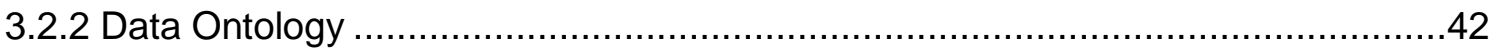

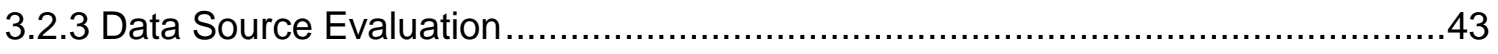

3.2.4 Data Fusion and Management Architecture ........................................................45

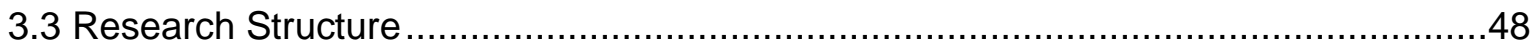

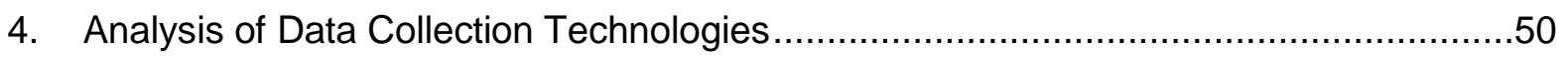

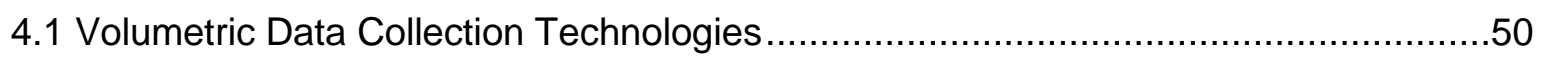

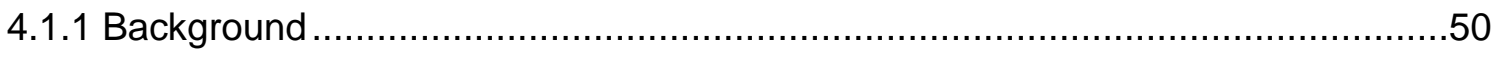

4.1.2 Experimental Program for Point Cloud Acquisition .........................................51

4.1.3 Labour hours for Data Collection and Data Processing …..................................53

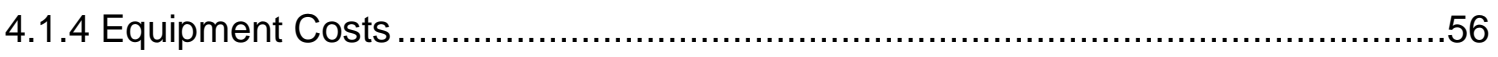




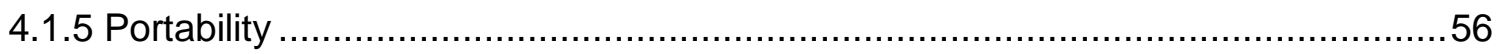

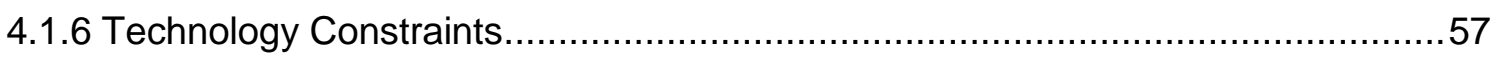

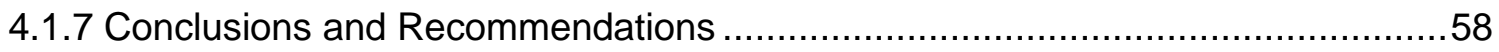

4.2 Positioning Technology: Ultra Wide Band (UWB) …………................................59

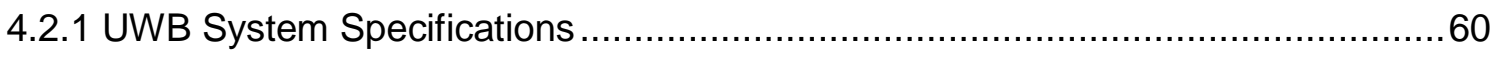

4.2.2 Phase I: Impact of Construction Materials on UWB System Performance ...........61

4.2.3 Phase II: UWB System Performance in an Active Indoor Construction

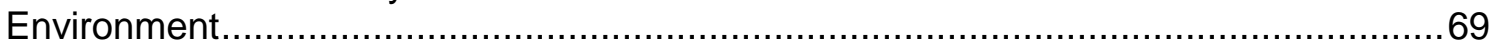

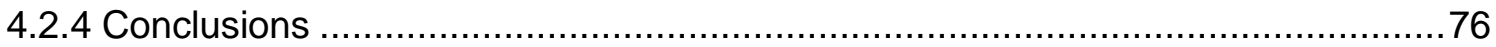

5. Data Fusion Model Development and Implementation ……....................................78

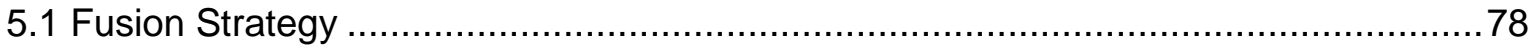

5.2 Field Setup and Data Collection ………............................................................79

5.3 Innovative Indicator-tag Approach for Activity Progress Tracking …............................83

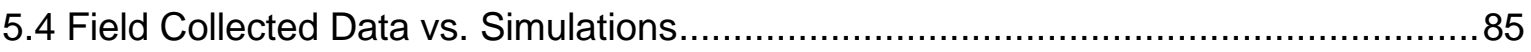

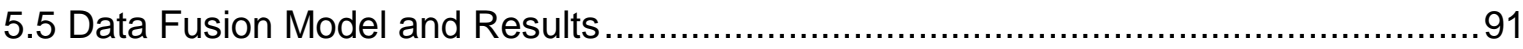

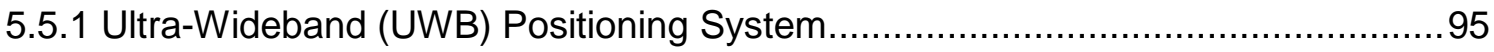

5.5.2 Material-Tracking Fusion ...............................................................................

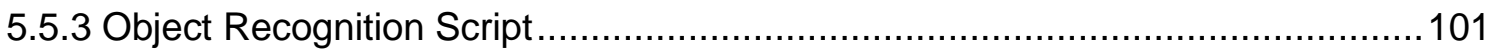

5.5.4 Path Generation for Indicator Tags ……………..................................... 102

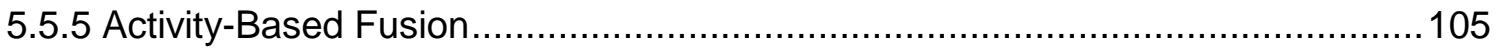

5.5.6 Project Control Applications ………........................................................119

5.6 Performance Evaluation of the Model ..............................................................124

5.6.1 Existing Progress Tracking Methods for Construction Sites ...........................125 


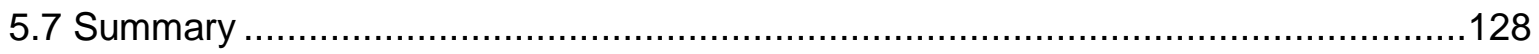

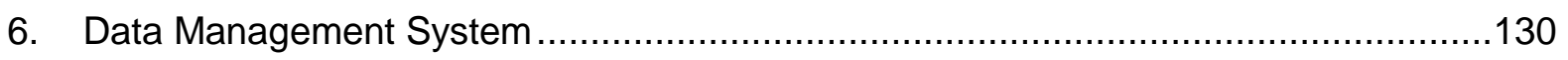

6.1 New Electronic Product and Process Management System (EPPMS) ..................131

6.1.1 Advantages of Electronic Product and Process Management Systems ............131

6.1.2 Coreworx Solution as the EPPMS Provider .............................................. 134

6.1.3 Objectives and Scope of the Developed EPPMS .......................................136

6.2 EPPMS Structure and Functionalities ........................................................137

6.2.1 Web-Based Project Portal .................................................................

6.2.2 Document Management System (DMS) ................................................138

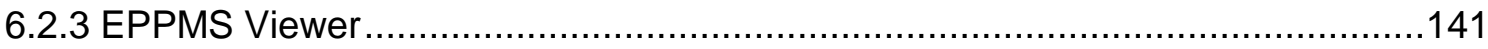

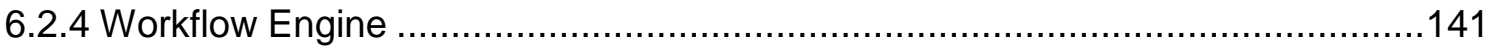

6.3 Workflows for Construction Research Data Management .................................141

6.3.1 Review and Approval of Inbound Transmittals ............................................142

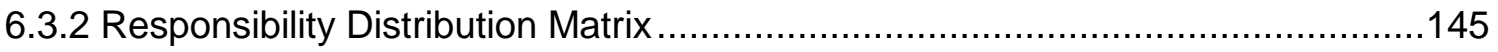

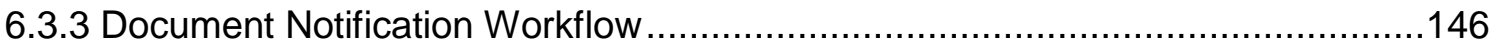

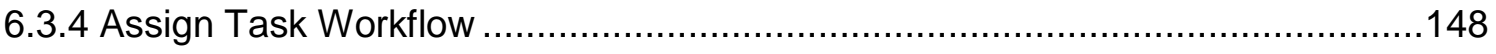

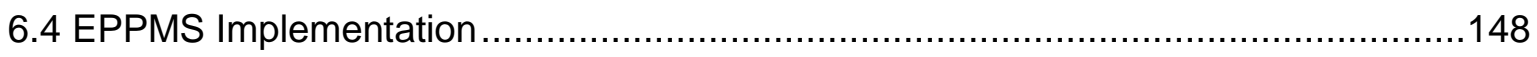

6.4.1 Step 1: Original Hierarchical Data Structure ............................................149

6.4.2 Step 2: Specification of Metadata Attributes and Generation of a Flat File.........150

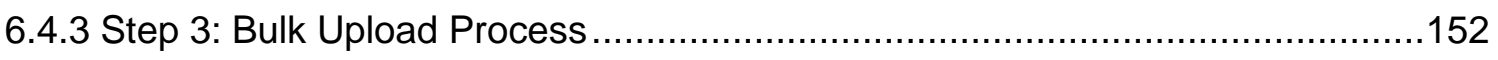

6.5 Integrated Data Fusion Workflow within EPPMS Framework ............................154

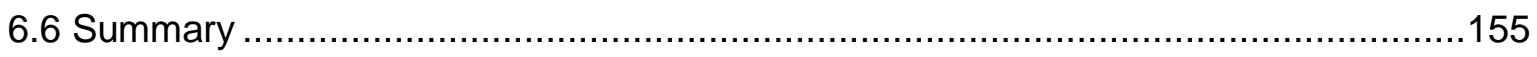




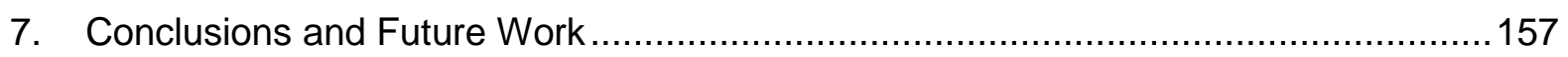

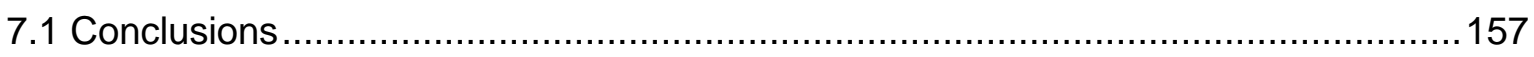

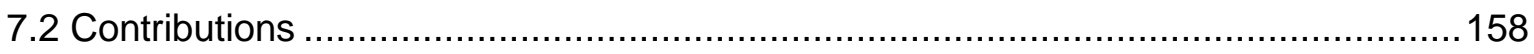

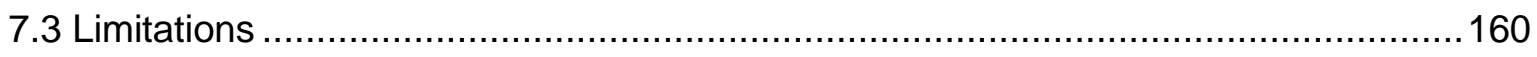

7.4 Outlook and Future Work ...................................................................... 161

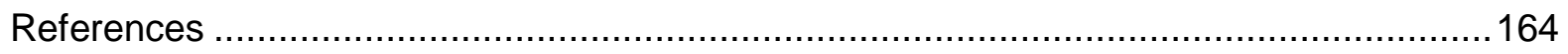

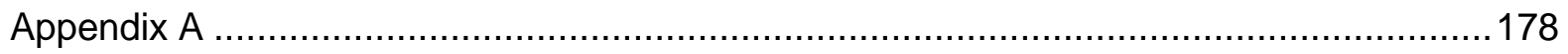

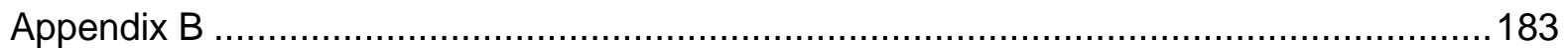

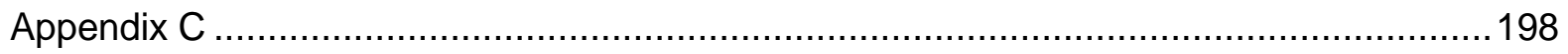

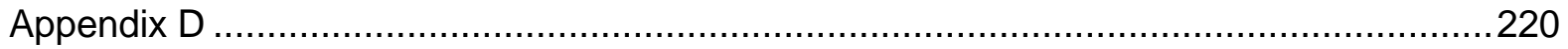




\section{List of Figures}

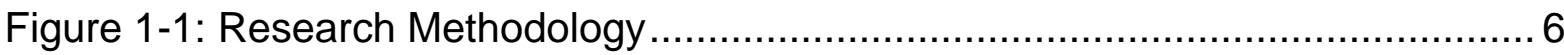

Figure 2-1: JDL Data Fusion Model Revised for Civil Engineering Applications ...... 23

Figure 3-1: Value Breakdown of E6 Piping Project ............................................. 43

Figure 3-2: Schematic Representation of the Developed Fusion Model ................. 45

Figure 3-3: Revised JDL Data Fusion for Construction Progress Tracking .............. 46

Figure 4-1: Sample Photogrammetry Results ................................................... 55

Figure 4-2: Sample Laser Scanning Results ................................................... 56

Figure 4-3: (a) Slim Tag, (b) Compact Tag, (c) Receiver ...................................... 62

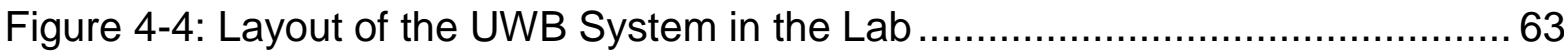

Figure 4-5: Plot of Cell Means for the Average Error ........................................... 67

Figure 4-6: Residuals Plotted Against Fitted Values ............................................68

Figure 4-7: UWB Scatter Plot for Wood and Metal Enclosures ..............................69

Figure 4-8: Site Development from the Installation (a) to the End of the Study (d) .. 70

Figure 4-9: Error Trend for Ground Points over Time .......................................... 71

Figure 4-10: Error Trend for Pipe Measurement Points over Time ......................... 72

Figure 4-11: Scatter Plots over the Project Duration for Selected Locations ........... 74

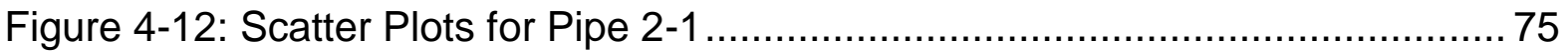

Figure 5-1: Data Fusion Processes within the Developed Model............................ 79

Figure 5-2: 3D CAD Model of Fifth-Floor Piping Layout (left) and Plan (right) .......... 80

Figure 5-3: Site Conditions at the Beginning of the Data Collection ....................... 81

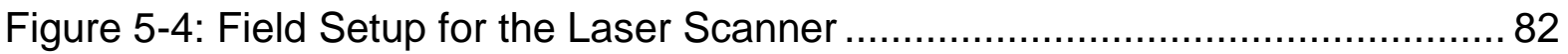


Figure 5-5: Schematics of the Activity-Tracking Data Capture .............................. 84

Figure 5-6: Hierarchical Structure of Activity-Based UWB Data .............................84

Figure 5-7: Elevation View of the Piping Network for Engineering VI Building .........85

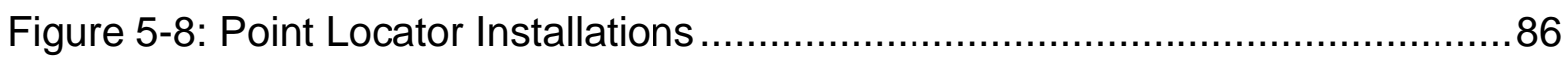

Figure 5-9: Connected Point Locator Readings …................................................ 87

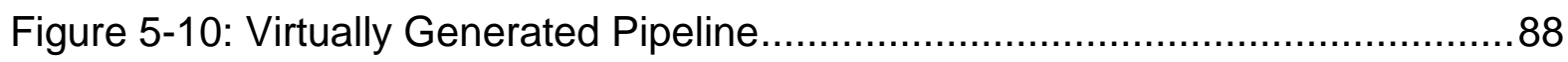

Figure 5-11: Simulated Pipeline as Used in the Model........................................8 88

Figure 5-12: Workflow Iconography Legend....................................................92

Figure 5-13: Activity-Based Fusion Architecture ...............................................93

Figure 5-14: Workflow for Site Implementation Research .......................................94

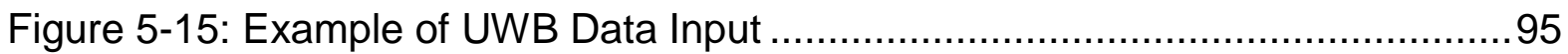

Figure 5-16: 3D Scatter of UWB Readings for One Point...................................... 96

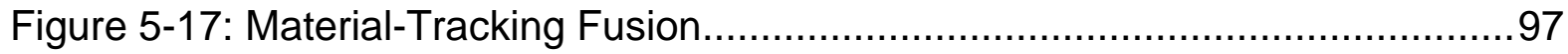

Figure 5-18: As-Planned Coordinates Extracted for the Pipeline ......................... 100

Figure 5-19: Function "Spoolerrorvalues" for Processing the Material-Tracking Files 101

Figure 5-20: Path Generation for Indicator Tags .............................................. 102

Figure 5-21: Snapshot of the File Format of the Activity-Tracking UWB Data........103

Figure 5-22: Activity-Based Fusion Engine ………......................................... 105

Figure 5-23: Percentage Design Change during the Life of Projects for Categories of

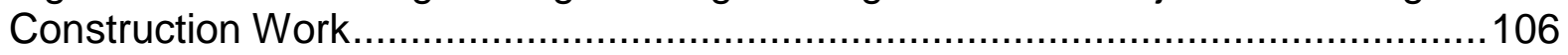

Figure 5-24: Error Trend for Pipe Measurement Points over Time.........................108

Figure 5-25: Sample Fuzzy Membership Functions ......................................... 109 
Figure 5-26: Schematic Representation of a Pipeline ...................................... 110

Figure 5-27: Schematic Drawing of Logical Operation Algorithms ....................... 114

Figure 5-28: Activity Fusion Based on Generated Path Data............................... 117

Figure 5-29: Graphical User Interface of the Fusion Model ................................ 120

Figure 5-30: Sample Contractor Payment Report ........................................... 121

Figure 5-31: Sample Original Schedule Export Used for Input ............................. 123

Figure 5-32: Sample Updated Schedule as Output.......................................... 124

Figure 6-1: Data Management within the Developed Fusion Model...................... 130

Figure 6-2: Structure of the Research Data EPPMS ........................................ 137

Figure 6-3: EPPMS Database Project Portal ................................................. 138

Figure 6-4: Review and Approval Workflow ................................................... 143

Figure 6-5: Sample Subset of a Responsibility Matrix...................................... 146

Figure 6-6: Document Notification Workflow ................................................... 147

Figure 6-7: EPPMS Implementation Process................................................... 148

Figure 6-8: Original Hierarchical Structure of the Engineering 6 Data .................. 149

Figure 6-9: Sample Flat File for Engineering 6 Building Data ............................. 150

Figure 6-10: Document Profile for Manual Input ................................................. 152

Figure 6-11: Sample Opened Document Profile ............................................... 153

Figure 6-12: Sample Search Results of the Model .......................................... 154

Figure A-1: The Graphical User Interface (GUI) ............................................ 178

Figure A-2: Data Priming and Pre-processing Logic ........................................... 179

Figure A-3: Sequence of Prompts for Materials Tracking Fusion......................... 180 
Figure A-4: Progress Date Prompt …...................................................... 181

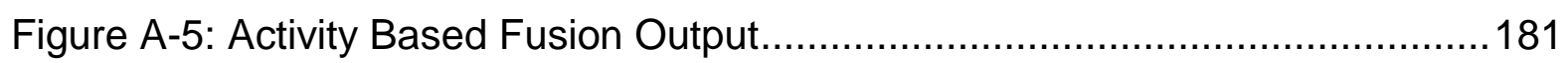

Figure A-6: A sample project schedule without engine modifications....................182

Figure A-7: Sample Duration Re-Calculations............................................... 182 


\section{List of Tables}

Table 2-1: Comparison of Radio Frequencies (Ergen et al., 2007) ........................ 14

Table 3-1: Potential Sources of Information ....................................................... 44

Table 4-1: Technical Specifications of the Laser Scanner ..................................... 52

Table 4-2: Technical Specifications of the Lens .................................................. 53

Table 4-3: Technical Specifications of the Camera.............................................. 53

Table 4-4: Specific Laser Scanner Resolution Characterizations ........................... 54

Table 4-5: Labour Hours for Data Collection and Processing ................................ 54

Table 4-6: Time Allocation for Laser Scanner Equipment Portability ......................57

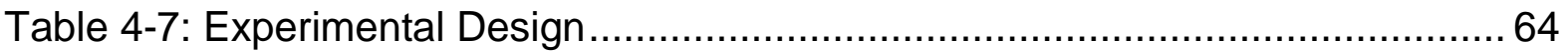

Table 4-8: Sample of Phase I Experimental Results for Average Error (m) ............. 65

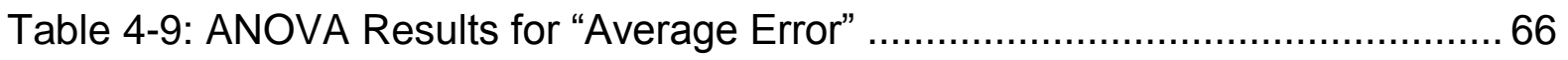

Table 5-1: Definition of Type I and Type II Errors ............................................... 98

Table 5-2: Generated Path Lengths and Errors .............................................. 104

Table 5-3: Sample Input Values for Line 1 ................................................... 111

Table 5-4: Sample Input Values for Line 2 …............................................... 111

Table 5-5: Sample Path Generation Results (from a given date) .......................... 115

Table 5-6: Sample Activity-Based Fusion Results for Line 1 (for a given date) ..... 118

Table 5-7: Sample Activity-Based Fusion Results for Line $2 \ldots \ldots \ldots \ldots \ldots \ldots \ldots \ldots \ldots \ldots \ldots . . .118$

Table 5-8: Sample Activity Progress Results ................................................. 119

Table 5-9: Sample Estimated Earned Value Results ......................................... 122 
Table 5-10: Schedule Adjustment Based on Estimated Progress

Table C-1: Error Summary for a Selection of UWB Readings during Field Experiments on UW Engineering IV Building

Table C-2: One of over 240 UWB Files Used from Field Data Collection on Engineering IV Building 200

Table C-3: True Coordinates of Fixed Points Captured Using Total Station Survey Equipment 214

Table C-4: Input Data for Four Lines for Installation Activity on October 30 2010214 Table C-5: UWB Generated Paths for Activity Progress Tracking for Four Lines on October $30^{\text {th }} 2010$ 216

Table C-6: Fusion Results for Installation Activity for Four Lines for Progress on October $30^{\text {th }} 2010$ 216

Table C-7: Intermediary Results for Progress of Four Lines on October 30 2010217 Table C-8: Final Activity Progress Estimates for October $30^{\text {th }} 2010$ 219 


\section{Introduction}

\subsection{Background and Motivation}

In recent years, many researchers have investigated the possibility of automating the tracking of the progress of a construction project. These efforts range from $2 \mathrm{D}$ photo feature extraction to the use of 3D laser scanners and radio frequency identification (RFID) tags (Bosché, 2010; Razavi, Young, Nasir, Haas, Caldas, Goodrum, \& Murray, 2008; Teizer, Caldas, \& Haas, 2007). However, most of the previous attempts at automating construction progress tracking have been developed based on a single-sensor model. With the continual development of data collection technologies, a significant amount of data can be collected at construction sites through a variety of sensing systems, but the information cannot currently be fully utilized without manually intensive post-processing procedures. A lack of the proper tools for storing, sharing, processing, and analyzing the data leads to most of the information collected being ignored by the site managers and decision makers involved in the project.

For informed decisions and objective assessments of the progress on a construction site, data from a number of sources must be combined because not all of the necessary information can be captured using a single data source. In recent years, a number of researchers have considered multisensor data fusion models in order to capture a more complete picture of the progress of a project by leveraging the information from Global Positioning Satellite (GPS) devices, RFID, and other sources of information for tracking and locating construction materials (Cheng \& Chen, 2002; Ergen, Akinci, East, \& Kirby, 2007; Moon \& Yang, 2010; Razavi, 2008; Razavi et al., 2008; Song, Haas, Caldas, Ergen, \& Akinci, 2006). Due to the volume and variety of the data sources and the lack of an effective data management system, most of the fusion structures reported in the literature are not scalable for large-scale construction projects with many sources of data.

Most existing data fusion models are based on data fusion at the sensor and object levels. In this research, this category of data fusion modeling is referred to as object-based data fusion (OBDF) models. In many applications, such as automated progress estimation, an OBDF process is insufficient because many project activities are not directly associated with a measurable physical entity at the site and therefore cannot be identified using traditional 
sensing techniques. These activities include concrete curing, installation, welding, inspection, and interior finishing. For example, with respect to piping activities, while OBDF models cannot differentiate an installed pipe from an installed, welded, and tested pipe, these designations correspond to completely different states of the project. A similar discrepancy applies to concrete pouring and curing related processes.

While a significant number of researchers have recently investigated the field of automated progress control for construction sites, in all cases the focus has been on large physical components, such as columns, beams, and floors (Bosche, Haas, \& Akinci, 2009). The construction industry could therefore benefit from an accurate, reliable, and efficient progress tracking method which is able to deal with both structural and non-structural activities on a construction site. As well, as demonstrated in this research, unlike existing object-based models, activity-based construction progress tracking would provide a much more compatible source of data for many construction management applications, such as earned value tracking and automatic schedule updates.

The fusion model developed in this research is based on a variety of sources of information collected by a number of stakeholders on a construction project. For the successful future implementation of this model, it was important that a system be defined to permit the sharing of data among the many stakeholders in a project. Also, recent advances in construction research are encouraging increased connectivity and communication in the construction industry. However, the developments behind these advances are typically not facilitated by a sophisticated communication and process management system for collaborative research. A lack of effective data management and data-sharing strategies remains one of the barriers to the development of collaborative and comprehensive data fusion models in the construction industry. A reliable and scalable data-sharing and data management system is therefore required in order to address these concerns, which can be viewed from two perspectives: the narrower one of data management of the enormous amount of data that was collected during this project, and a wider global viewpoint of the sharing of data with the entire construction research community. 


\subsection{Research Objectives}

To provide an efficient, accurate, reliable, and scalable data fusion model for the purposes of automated construction progress tracking, three main objectives were defined in this research:

1) To investigate and evaluate potential sources of, and technologies for, onsite data collection for particular application in the automated progress tracking of construction projects. This objective was included to quantitatively characterize the input data sources. This information was required for the development of the fusion algorithms.

2) To develop an activity-based data fusion model for the assessment of the progress of activities on a construction site, with an emphasis on the activities associated with nonstructural trades. Within this main objective, the following sub-objectives were identified:

- Provide a workflow-driven approach for the efficient, accurate, and reliable estimation of progress in construction projects. The approach should be as automated as possible, in order to reduce human error and eliminate bias from the estimates.

- Fuse data at the highest levels of data fusion models in order to assist with decision-making and project management.

- Assess both structural and non-structural activities on construction sites.

- Fuse data from sensory sources such as RFID and ultra-wideband (UWB) systems as well as information from non-sensory sources such as inspection, schedule, and progress reports.

3) To establish a reliable, efficient, and scalable workflow-driven data management system for sharing construction research data, from which a variety of data fusion models could extract the data required. This data management system should be able to perform the following functions:

- Manage all types of construction research data and ensure the quality, integrity, and safety of all files within the database.

- Allow the effective and secure sharing of research data with other research institutions around the world. 
- Provide a platform so that data fusion models, including the model developed in this research, can extract the data sources required.

- Allow all stakeholders and consultants to share their corporate as well as research data with the research community.

\subsection{Research Scope}

No existing fusion architecture for the activity-based progress tracking of construction projects was reported in the literature. The research presented in this thesis was therefore directed at establishing benchmarks and industry-accepted standards against which future work in this area could be evaluated. While the developed fusion architecture is as general as possible and flexible enough for a wide range of activities and projects, the model presented in this thesis was designed for the piping activities associated with an industrial-type building project. Piping systems were chosen because they include both activities directly associated with physical entities, such as delivery and installation, and other non-structural activities, such as welding and inspection. The expansion of the proposed model to cover different construction activities and projects is outside the scope of the present research and is provided only as a recommendation for future research.

For construction sites, the effort required in order to implement any sensing or monitoring system directly governs the practical applicability of such a system. Therefore, data fusion processes must be as formalized and as automated as possible in order to be considered a practical tool for construction project managers. The automation developed in this research is limited to the workflows and fusion processes, and some manual data collection techniques are still required. Further automation of the data collection processes is suggested as a possible area for future research.

A data fusion model that incorporates a wide range of input information also needs a reliable and scalable data management and sharing system. The scope of the developed data-sharing and management system is limited to the data collected for this particular project, but strategies are recommended for the further expansion of the model to include the entire construction research community. 


\subsection{Research Methodology}

The research presented in this thesis was motivated by the inability of existing data fusion models to effectively address the problem of automating the evaluation of the progress of construction activities. The methodology of this research is shown schematically in Figure 1-1. The first step in this research was a review of the literature related to data fusion as applied to the monitoring and estimating of construction progress. The sources of sensory information available on construction sites were also investigated: RFID systems, UWB positioning systems, 3D laser scanners, photogrammetry, barcode systems, building information models (BIMs), and GPS. The literature review was focused on the existing individual and integrated applications of these technologies on construction projects for the purposes of tracking construction progress. The research vision was then developed based on the knowledge gaps identified from the literature.

In parallel with the development of the fusion architecture, the existing state-of-the-art technologies to be used in the data fusion model were also evaluated. A comparative study was conducted in order to evaluate photogrammetry and 3D laser scanners for the purposes of point cloud acquisition, based on which automated object recognition algorithms could be employed. Also, the performance evaluations of UWB positioning systems reported in the literature were inconsistent and incomplete with respect to the application of this technology in construction environments. A detailed experimental program was therefore designed in two phases as a means of evaluating the performance characteristics of UWB positioning systems in indoor construction environments.

As the data collection strategies and technologies were evaluated, the fusion architecture was also being developed. The activity-based data fusion model that was created in this research was validated in part through simulations and in part through field experiments based on the data collected over several months from a building construction project on the University of Waterloo campus. The validation of the model had to be conducted in instalments because the model was being developed and calibrated simultaneously with the data collection stage. For this reason, part of the validation was also performed using functional proofs or functional validations, as explained in greater detail in Chapter 5 . The building in question housed chemical engineering laboratories and therefore contained electrical, piping, and HVAC services 
that are similar to those found in an industrial or hospital project. The scope of the validation was limited to the piping work of the building.

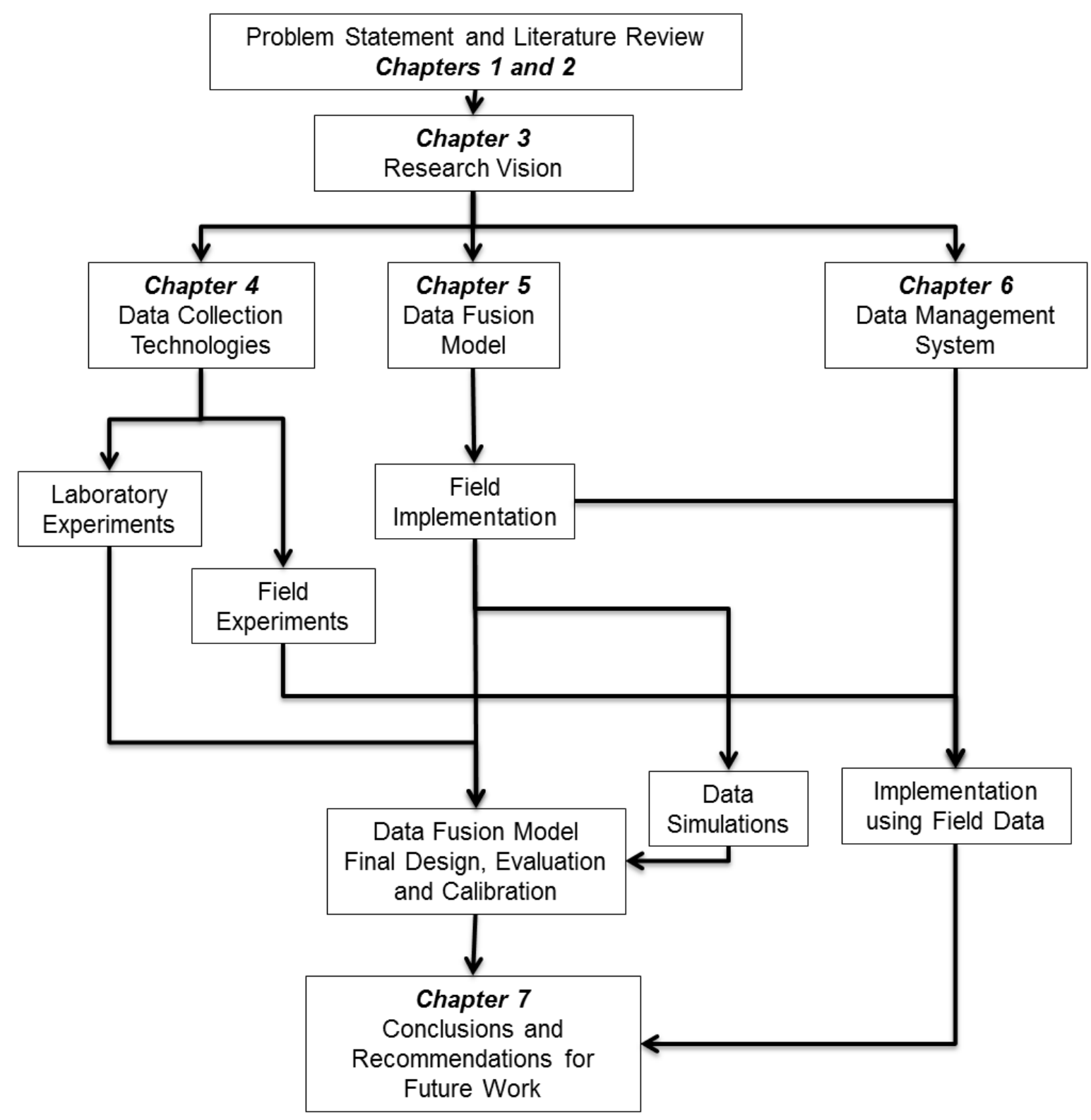

Figure 1-1: Research Methodology

A vast amount of data was collected for this research project, which can be used for many future studies in the construction progress estimation domain as well as many other related domains. It was recognized that the multi-dimensional nature, level of detail, continuity, and 
comprehensive aspects of the data collected for this research would be suitable for many aspects of construction research but only if an effective research data sharing and management system was available. Therefore, as the final stage of the current research, a reliable, efficient, and scalable data management system was developed in order to enable the sharing of the construction research data. The system was implemented and validated using the data collected during the various stages of the current research.

\subsection{Structure of the Thesis}

This thesis is organized in seven chapters. Chapter 1 presents an overview of the research: the background and motivation, research objectives, scope of the research, and research methodology. Chapter 2 summarizes the literature related to automated construction progress tracking, applicable sensory sources of information, and multi-sensor data fusion models. The studies discussed include previous work with respect to bar coding systems, RFID systems, GPS, UWB positioning systems, and BIMs in the context of the tracking of construction progress. Chapter 3 describes the knowledge gaps evident from the existing literature and then describes the research vision, approach and structure. Chapter 4 presents a summary of the evaluation of the volumetric data collection technologies and the results of a detailed investigation of an Ultra Wind Band (UWB) positioning system as an indoor positioning system. Chapter 5 presents the developed data fusion architecture, including workflows, algorithms, and processes. This chapter also summarizes the field experiments on a construction project in Waterloo that was used for developing various algorithms within the model. The data management and sharing system that was developed during this research is then explained in Chapter 6, along with the implementation of the system using the data collected during the current research. Finally, Chapter 7 includes the conclusions, contributions, and limitations of this research, as well as recommendations for potential future research opportunities.

There are four appendices included in this thesis. Appendix A presents a step-by-step guide for the software that was developed for data fusion process of this research, with the entire code presented in Appendix B. Appendix C presents a small selection of the input data that was used in this research and finally Appendix $D$ presents a summary of the abbreviations and terminology that is used in the thesis. 


\section{Literature Review}

\subsection{Introduction}

The automated tracking of construction progress has been investigated by many researchers who have employed a variety of technologies, methods, and concepts. Most of these studies have focused on the introduction and implementation of one specific sensing technology for construction projects. In recent years, a number of researchers have taken advantage of the complementary capabilities of these technologies. This chapter presents the latest research and describes the state-of-the-art with respect to automated construction progress tracking, followed by a brief overview of studies related to a number of specific technologies in the context of the automation of the tracking of construction materials and progress.

\subsection{Automated Construction Progress Tracking}

In the past, automated production tracking has been successfully implemented in industrial manufacturing and processing plant applications. However, due to the temporary nature of construction sites, standard industrial monitoring systems and strategies are not suitable for automated construction progress tracking (Sacks, Navon, Brodetskaia, \& Shapira, 2005). The monitoring strategies currently used in the construction industry are labour intensive and result in inaccurate, inefficient, and incomplete data (Bosche \& Haas, 2008; Sacks, Navon, \& Brodetskaia, 2006).

A number of research studies have involved the use of information from Global Positioning Satellite (GPS) devices, radio frequency identification tags (RFID), and other sources of information for tracking and locating construction materials (Cheng \& Chen, 2002; Ergen et al., 2007; Moon \& Yang, 2010; Razavi et al., 2008, Song et al., 2006). Ergen (2007) investigated the use of RFID tags for tracking materials as well as for facility management systems. Razavi (2008) developed a location estimation algorithm for RFID tags for locating pipe spools in a laydown yard. Cheng and Chen (2002) integrated a barcode system with wireless radio frequency transmission technology for a real-time construction monitoring application. Teizer (2007) fused data collected from 3D range cameras for detecting and tracking equipment and 
crews on construction sites, which could enable a range of other applications in construction sites, including effective management practices for improving safety on construction job sites.

With the development of all of these technologies, a large amount of data can be collected on construction sites on semi-automatic and semi-continuous bases. However, processing and reducing data to meaningful conclusions and fusing data from different sources remain as obstacles to the achievement of a practical and comprehensive automated progress tracking solution for construction sites. Although most of the research in the area of automated construction progress tracking has been technologically driven and limited to the tracking of materials and equipment, Navon and Sacks (2007) have proposed an alternative approach based on crew productivity. With their method, the needs of the management team and decision makers are evaluated for a specific construction project, and the data acquisition technologies that best suit those specific needs are then chosen, thus reducing the amount of data collected (Navon \& Sacks, 2007). This approach focuses on crew productivity and crew tracking, which remains a controversial topic in most countries.

The state of the art in technological advances with respect to data collection and the large amount of multisensory data collected on construction sites have motivated a number of researchers to investigate and develop data mining and knowledge discovery tools, processes, and techniques in order to discover new knowledge by searching and organizing large construction databases (Soibelman, Wu, Caldas, Brilakis, \& Lin, 2008). They noted the problem of the increasing volume of data collected on construction sites and focused on the management of unstructured information, such as text files and pictures from the sites.

The research presented in this thesis has also identified the problem of recording large amounts of information from different sources and the inability of existing models to take full advantage of the information available. The fusion model presented in this thesis provides a platform for fusing data from different sources for the purposes of decision making in the context of automated construction progress tracking. A suitable, efficient, and reliable data management system has also been developed as a means of addressing many of the concerns of research and industry partners in this field with respect to the management and sharing of research data. 
The next three sections of this chapter summarize the existing published literature related to a variety of data collection technologies for construction progress tracking: object recognition from 3D point clouds, barcoding and RFID, and GPS and UWB. A review of BIM requirements and integration possibilities follows, and then existing data fusion models and taxonomies are reviewed.

\subsection{Object Recognition from 3D Point Clouds}

Analysis of 3D point clouds is an effective method of object recognition that can be successfully applied to construction progress tracking. Point clouds can be obtained through either close range photogrammetry or laser scanning technology.

The art and science of photogrammetry, or metrophotography as it was originally termed by its inventor, Laussedat, in 1851, was developed as a means of determining from ordinary photographs the correct metrical representations of the object photographed (American Society of Photogrammetry, 1980). Aerial photogrammetry was used extensively in the 20th century because of the importance of measuring topographies for a variety of considerations, ranging from strategic military to real estate and environment conservation.

Close range photogrammetry usually refers to that branch of photogrammetry that covers distances of less than 300 metres between the object and the camera. Close range photogrammetry has been applied in a wide variety of disciplines including manufacturing, medicine, sports, biology, zoology, aerospace, forensic science, and the preservation of cultural heritage sites. In the civil engineering domain, it has been used for structural monitoring, and research initiatives are being conducted to determine its suitability for automated construction progress monitoring (El-Omary, 2008; Ahmed et al., 2011c).

With current advances in photogrammetric technology, it has become possible to assemble a low-cost system that works with any off-the-shelf high-resolution camera and any off-the-shelf software (SW) that handles camera calibration, interior and exterior orientation, bundle adjustment, image normalization, and epi-polar stereo matching. Photogrammetric SW is now also able to generate a high-density point cloud automatically, and using photogrammetric technology to acquire 3D point clouds has thus become feasible. Object recognition from digital 
photogrammetric data has been investigated for use in the detection of structural elements on a construction site, and the recognition results have subsequently been used for tracking construction progress (Kim, Son, \& Kym, 2011)

The second method of acquiring 3D point clouds is a terrestrial three-dimensional laser scanning technology, also called Laser Detection and Ranging (LADAR). Automatic object recognition techniques from $3 \mathrm{D}$ laser scans have been developed for project management and quality control applications. Recent developments in object recognition techniques have enabled the retrieval of 3D computer-aided design (CAD) objects from laser-scanned data (Bosche \& Haas, 2008; Bosche, Haas, \& Murray, 2008; Bosche et al., 2009).

Bosche's (2009) new approach to the recognition of 3D CAD objects from point clouds obtained from 3D laser scans of a site has enabled a wide range of applications in the construction industry. In this method, the 3D CAD model is registered (or referenced) in the scan's spherical coordinate frame. Then, for each as-built range point in the point cloud, a corresponding virtual range point or as-planned point is calculated by using the scan referenced project 3D model as the virtually scanned world. Using this strategy, each point in the as-planned range point cloud corresponds to exactly one point in the as-built range point cloud. However, in the virtual scan, it is known from which 3D model object each as-planned point is obtained. Finally, the recognition of each object is performed by considering the number of its recognized as-planned points.

Researchers have used the possibilities offered by the laser detection and ranging (LADAR) technology to monitor land sliding and soil deformation measurements. LADAR technology has also proven a valuable aid for construction managers with respect to a variety of tasks such as material tracking, progress monitoring, quality control, and facility/infrastructure management (Kiziltas, Akinci, Ergen, Tang, \& Gordon, 2008). In the same vein, Golparvar-Fard et al. (2009) note that such a tool allows managers to remotely explore a construction site and it can also be used for contractor coordination. Laser scanning technology has also been employed for the analysis of surface flatness, quality assurance, floor plan modeling, and recognition of building components (Huber et al., 2010). All of these examples demonstrate the extensive range of applications for laser scanning technology, and thus the associated need for a reliable and costeffective method of point cloud acquisition for construction sites. Chapter 3 presents a detailed 
analysis and comparison of these 3D laser scanning and photogrammetry technologies for the purposes of automated object recognition from 3D point clouds.

In addition to enabling remote and automated applications for tracking progress and productivity, object recognition results could also be used for remote design and diagnostics, automated identification of safety code violations, and the remote quality assessment and quality control of construction dimensions. Therefore, improving the results of existing object recognition methods by incorporating activity-based data fusion can be helpful in a wide range of applications in the architectural/engineering/construction and facility management (AEC\&FM) industry.

One of the objectives of this research was to evaluate the data collection methods that could be used on construction projects to capture the data required for a reliable, efficient, and accurate progress estimate. While object recognition methods from 3D point clouds have demonstrated very promising results in controlled environments, many other sources of information are available or could be easily obtained from construction sites and could be combined in order to improve the results and present a more robust model for recognizing objects from a 3D laser scan of a site. These sources of data include RFID and UWB tags which are discussed in the following sections. The goal of this research was to leverage the benefits of this variety of sources of information as well as the schedule information in the development of the data fusion architecture.

\subsection{Bar Coding and RFID}

The level of object recognition available from 3D point clouds can provide information about the shape, location, and orientation of objects on a site; however, significant human post-processing is required for information about the nature or history of an object to be extracted (Razavi et al., 2008). As well, with Bosche's approach (Bosche et al. 2009) and any other approach that uses a 3D model as a-priori information, an object is recognized only if it is exactly at the location predescribed by the 3D CAD as-designed model. Therefore, these methods are unable to identify objects that have been delivered to the site and not been installed, or objects that have been installed but are separated from their designed location by a distance greater than the tolerance specified in the object recognition software. Sources that use 3D methods are also currently 
incapable of providing any information regarding the non-structural activities on a site, such as the welding or testing of a piping system. Building Information Model (BIM) systems may facilitate this capability in the future.

Tracking technologies form the backbone of any automated construction progress estimation system and are generally based on bar coding or RFID Tags. RFID has been investigated since the mid-1990s as a promising technology for tracking construction materials. Bar-coding techniques for tracking materials and equipment were the first attempt at the use of identification tags on construction sites. Bar-coding techniques have been investigated as a means of providing an effective approach for improving procedures for timekeeping and tracking material quantities at a construction site (Rodriguez \& Jaselskis, 1994).

There are two main differences between RFID and bar-coding technologies in terms of tracking applications: bar-coding requires a direct line of sight between the tag (or label) and the reader, and RFIDs are capable of providing 2D or 3D position information semi-automatically and semicontinuously. Since RFID tags do not require a line of sight, they can be more easily adapted to construction site applications where they can be embedded in material packaging or weatherproof cases for greater durability. Another advantage of RFID technology is that each tag has a distinct ID, which means that each piece of equipment or material can be tracked independently. While barcodes use a global standard, they can be used only to identify the manufacturer and product and cannot differentiate between different replicates of a product. A standard bar code system allows different parties to use the same system, an advantage that current state-of-the-art RFID technology lacks because it is a proprietary technology and can be used by different parties only if they all use a product from the same vendor. Table 2-1 summarizes the capabilities associated with the frequencies of commercially available RFID systems. As shown in this table, some RFID tags have Read Only (RO) functionality, while others could have both Read Only (RO) and Read and Write (RW) capabilities. Because construction projects often involve a need for long-range active tags, systems based on UHF frequencies are often employed (Ergen et al., 2007). 
Table 2-1: Comparison of Radio Frequencies (Ergen et al., 2007)

\begin{tabular}{|c|c|c|c|c|}
\hline Frequency & Typical Tag Type & Range & Reading Speed & Memory \\
\hline Low: $125 \mathrm{KHz}$ & Passive, Mostly RO & Short-Medium & Low & Small \\
\hline High: $13.56 \mathrm{MHz}$ & Passive, Mostly RO & Short-Medium & Medium & Small \\
\hline UHF: $868 / 928 \mathrm{MHz}$ & $\begin{array}{l}\text { Active/Passive, } \\
\text { RO/RW }\end{array}$ & Long & High & $\begin{array}{l}\text { Small- } \\
\text { Large }\end{array}$ \\
\hline $\begin{array}{l}\text { Microwave: } 2.45-5.8 \\
\text { GHz }\end{array}$ & $\begin{array}{l}\text { Active/Passive, } \\
\text { RO/RW }\end{array}$ & $\begin{array}{l}\text { Long- } \\
\text { directional }\end{array}$ & High & $\begin{array}{l}\text { Small- } \\
\text { Large }\end{array}$ \\
\hline
\end{tabular}

The many advantages of RFID technology have attracted a number of researchers who have investigated the application of this technology in construction projects. These studies include a feasibility analysis of using RFID technology for automatically identifying and tracking individual pipe spools in laydown yards and under shipping portals (Song et al., 2006); an examination of data acquisition for tracking work progress on construction sites (El-Omari \& Moselhi, 2009a); an investigation of automated material tracking in construction (Razavi et al. , 2008) and an analysis of the monitoring and controlling of construction operations, such as a concrete pouring operations (Moon \& Yang, 2010).

Both RFID and UWB tags, discussed in the next section, can be used to track the locations and movement of materials, equipment, and components on construction sites, and the information they provide can complement object recognition results from 3D point clouds through the use of location estimation algorithms. However, unlike 3D point clouds, RFID and UWB tags can be used in non-conventional ways in order to track non-structural activities, as demonstrated in the research presented in this thesis.

\subsection{GPS and UWB}

GPS was the first, and is currently the only, fully functional Global Navigation Satellite System (GNSS). The GPS system has been used by the United States Department of Defence (DOD) as a military application since the 1980 s and has been available at the consumer product level since the 1990s. In recent years, the cost of GPS technology has been sufficiently reduced to the point where its use for construction projects can be justified, and a number of researchers 
have investigated its advantages for material tracking, construction progress estimation, mining, earthmoving, and many other applications.

Because GPS technology provides accurate global coordinates that can be easily integrated with other sources of information, it is now being used on drills, graders, loaders, dredgers, shovels, and excavators as a means of increasing accuracy, reducing contamination and disturbances, and improving site safety (Seymour, 2007). The use of conventional and GPSbased systems for earth moving operations has also been compared and an increase of $22 \%$ in productivity and cost savings of $13 \%$ were found with the GPS-based system (Han, Lee, Hong, \& Chang, 2006). GPS systems have also been investigated with respect to their use in construction in conjunction with other technologies, such as 3D laser scanning and RFID tags (Song, Haas, \& Caldas, 2007).

Although GPS technology has proven to be a powerful tool and a valuable addition to other sensing and tracking technologies currently employed in construction projects, its application is limited to outdoor projects because its signal degrades substantially in most indoor environments. There is therefore a need for a location estimation system similar to GPS technology but which would be able to accommodate indoor construction environments. Ahmed and Hegazy (2008) conducted a thorough analysis and comparison of the available positioning technologies that could be used for indoor and outdoor environments. The positioning systems that were examined included RFID, network based, infrared, ultrasound, inertial navigation, satellite, pseudo-satellite, and hybrid. It was concluded that in applications where the environment is not confined, the cost of the installation of permanent RFID or UWB readers was not justified, and other solutions, such as enhanced GPS systems, were more appropriate (Ahmed \& Hegazy, 2008). However, in construction sites, where the limits of the site do not change, and the environment can therefore be confined by pre-set limits, the use of networkbased systems, such as wireless LAN, Bluetooth, and ultra-wideband (UWB), could be investigated. Bluetooth technology for positioning systems is currently limited to low-range applications and is thus not suitable for construction projects. Wireless LAN or UWB was therefore deemed more appropriate for the research presented in this thesis.

UWB technology has emerged as an effective real-time location-sensing and resource-tracking technology. A UWB system is a network of receivers and tags that communicate with one 
another over a large bandwidth (>500 MHz). Each tag transmits UWB radio pulses that enable the system to find its 3D position coordinates. UWB technology dates back to the 1960s; however, the last decade has seen an acceleration in its development and the investigation of its application in the construction industry. Prior to 1994, UWB research was restricted to U.S. government programs and secure military radio and intrusion detection systems, since the short pulses of the generated signal result in very accurate timing information (Gu \& Taylor, 2003). The Federal Communications Commission (FCC) increased non-government-related research after 1994, but it was not until 2002 that the FCC approved the unrestricted use of low-powered UWB systems and tags ( $5 \mathrm{~mW})$. Therefore, although UWB technology has been available for a considerable time, its successful application in research projects has been limited to the last decade. Another factor contributing to the recent popularity of UWB technology is the declining cost of microchips due to advances in application-specific integrated circuits and complementary metal oxide semiconductors (Gu \& Taylor, 2003; Khoury \& Kamat, 2009; Teizer et al., 2007).

The advantages UWB technology over other tracking and positioning technologies include the following:

- Very low power, making it ideal for specific environments that are sensitive to radio frequencies such as hospitals and health care facilities

- Longer read ranges than GPS, laser scanning, or vision-based detection and tracking systems (up to $1000 \mathrm{~m}$ )

- No requirement for line-of-sight to the receivers

- No requirement for satellite or base station connections

- The ability to operate both indoors and outdoors

Material tracking is one of the more obvious applications of UWB technology for construction sites and has attracted significant industry attention from both a management and a progress control perspective as well as with respect to site searches in laydown yards. RFID systems have been extensively researched as a material tracking improvement tool for the construction industry, and a wide range of applications have been developed (El-Omari \& Moselhi, 2009a; Motamedi \& Hammad, 2009; Razavi, Haas, Vanheeghe, \& Duflos, 2009; Sacks et al., 2006). However, RFID systems must be integrated with GPS or other technologies in order to provide 
an accurate 3D location estimate. UWB can replace an integrated system of RFID and GPS, which has been developed and successfully implemented by Razavi et al. (2009). UWB is preferable to an integrated RFID-GPS system for some applications, despite its relatively high setup costs, because it can be used both indoors and outdoors.

The distinct advantages of UWB technology make it ideal for a variety of applications on construction sites, either independently or as part of an integrated system with one or more of the other available tracking and monitoring technologies. Potential construction-site applications of UWB technology, in addition to material tracking discussed earlier, include the following:

- Proactive work-zone safety can be achieved by installing tags on crew members and equipment on the site and by monitoring the distances between them. Such a system could provide notification of a situation that could be hazardous to either a crew member or an equipment operator. The UWB systems currently commercially available allow twoway communication between the tags and the data acquisition system and even have a built-in buzzer functionality, which enables the tag to be paged through the data acquisition system. Rescue operations can also be included in work-zone safety (Teizer, Venugopal, \& Walia, 2008). Knowledge of the location of crew personnel at any given point increases the potential for a rescue operation to be automatically analyzed in real time.

- Workforce training can include the use of UWB and has already done so in military applications. The movements of soldiers are tracked through UWB tags and then are analyzed and used for training soldiers with respect to better positioning and manoeuvring in a given situation. The same concepts can be applied to the construction workforce through the use of tags on trucks, excavators, and other onsite equipment to provide feedback regarding site-planning and process optimization.

- Decision-making processes can be significantly more effective if accurate and archived UWB information is available to indicate the location and movement of materials, equipment, and crews at any point in time. The post-processing of the data can then identify bottlenecks and laydown yard problems. 
In any real-time positioning system application, the accuracy of the readings is very important, and therefore understanding the performance behaviour of the positioning system in different environments is critical. The published research on UWB positioning systems reports accuracies ranging from $0.1 \mathrm{~m}$ to $2.0 \mathrm{~m}$, typically based on controlled laboratory experiments. Most of the studies reported in the literature investigated situations that involved direct line of sight and sometimes partial occlusions (Cheng, Venugopal, Teizer, \& Vela, 2011; Cho, Youn, \& Martinez, 2010; Gu \& Taylor, 2003; Teizer et al., 2008). Cho et al. (2010) investigated the overall performance of a UWB system in steel versus wood-framed construction environments, although complete occlusion of the tags (i.e., lack of line-of-sight) was not considered. Cheng et al. (2011) investigated the performance of a UWB system in real construction projects for safety and productivity purposes; however, the study area did not include significant occlusions or obstructions, and remained static during the study period.

There are no established or accepted standards for the evaluation of UWB positioning systems that are currently commercially available. Because published studies related to the accuracy and sensitivity of UWB systems in construction environments are limited, and since one of the objectives of the current research was to evaluate potential sources of data from construction sites, an entire experimental program was built into this research in order to investigate the performance of a UWB positioning system in construction environments, which is presented in Chapter 4 of this thesis. As reported in Chapter 4, the study was based on a systematic and statistical approach to the evaluation of a UWB positioning system, which was then used extensively in the data fusion model developed in this research.

\subsection{Integration with BIM}

The National BIM Standard defines a Building Information Model (BIM) as a digital representation of the physical and functional characteristics of a facility (Eastman, Teicholz, Sacks, \& Liston, 2008). A BIM is a shared knowledge resource for information about a facility that forms a reliable basis for decisions during its life cycle, which is defined as existing from the earliest conception to demolition. A basic premise of a BIM is collaboration among different stakeholders at different phases in the life cycle of a facility, which involves the insertion, extraction, updating or modification of information in the BIM to support and reflect the roles of 
that stakeholder. The AEC\&FM industry is highly fragmented, and a platform for the exchange of knowledge and for collaboration purposes is therefore highly desirable.

A BIM is an accurate three-dimensional digital model of a building. One of the advantages of a BIM over a 3D AutoCAD format is that the objects in the BIM are parametric, are linked to each other, and contain a variety of attributes. Parametric objects consist of geometric definitions as well as associated data and rules. For example, when a light switch is inserted into the plan for a particular room, it is automatically placed at the correct side of the door, or when the height of a ceiling is increased, the walls automatically resize themselves in order to maintain the prescribed definition of the room. When the size of an entire building is modified, the parametric properties of the elements within the model can also support recalculations of the HVAC system requirements in seconds.

Ideally, a BIM would be created at the design stage; would then be modified and completed at the construction stage; and for management and maintenance purposes, would stay with the building for the entire life of the building. This long-term advantage of the BIM may justify the permanent attachment of sensors, such as RFID tags, to a number of key components (Motamedi \& Hammad, 2009). The permanent RFID tags can be used for material tracking at the manufacturing and delivery stages (Razavi et al. 2008), for progress tracking during the construction stage, and for maintenance during the entire life cycle of the component. Motamedi and Hammad (2009) developed a conceptual BIM-RFID system that would take advantage of permanent RFID tags on a variety of components. In this conceptual system, the RFID number is assigned to each component at the design stage; the RFID tag is placed at the manufacturing stage; and the tag is then scanned at various times throughout the shipping, receiving, storage, assembly, installation, inspection, and operation stages. This conceptual and idealistic model entails a number of practical challenges related to the current practice standards in the AEC\&FM industry. From a contractual perspective, the ownership of the BIM and the associated liabilities and responsibilities need to be explicitly defined because the intelligent use of a BIM will cause significant changes in the relationships among the parties involved in a construction project.

Despite the implementation and legal challenges associated with BIM, many owners are now requesting that builders and contractors provide them with a comprehensive BIM as part of the 
construction contract. The General Services Administration (GSA) of the US federal government demands a BIM model that can automatically check to determine whether the design meets program requirements (Eastman et al., 2008). Based on the growing popularity of BIMs among owners and the many advantages that they provide for all parties involved in a project, it is clear that BIMs will be playing a significant role in the future of the AEC\&FM industry.

The level of integration of the research for this thesis with BIM models is limited to data extraction with respect to 3D model elements, which was essentially established using a 3D AutoCAD model. The variety of applications and output generated by the current research, such as as-built information, updated schedules, and progress estimates, can be communicated back to the BIM model. Further integration with BIM models was outside of the scope of this research but is recommended as a logical next step for future research in this area.

\subsection{Multisensor Data Fusion}

Multisensor data fusion refers to the combining of information from one or more sources in order to improve on the quality of the information obtained separately from each source. In the original Data Fusion Lexicon, the Joint Directors of Laboratories define data fusion as "a process dealing with the association, correlation, and combination of data and information from single and multiple sources to achieve refined position and identity estimates, and complete and timely assessments of situations and threats, and their significance. The process is characterized by continuous refinements of its estimates and assessments, and the evaluation of the need for additional sources, or modification of the process itself, to achieve improved results" (White, 1987). Steinberg et al. (1999) provide a more concise definition of data fusion as "the process of combining data to refine state estimates and predictions." A number of other definitions of data fusion have been proposed; however, for the purposes of this research, the definition provided by Steinberg et al. serves as a useful concise definition of multisensor data fusion. In this research, multisensor data fusion is utilized to make inference decisions about the state of a construction project based on data from different sources.

The broad concept of data fusion has been studied extensively in many research areas for a number of decades, which has resulted in mixed and confusing terminologies. The confusion regarding the term "data fusion" occurs because of the two main data fusion cultures within the 
data fusion community (Dasarathy, 1997). One culture is based on sensor fusion, which deals with data fusion at the lowest levels, as explained in this chapter. The research projects in this area deal with raw data that have been obtained from physical sensors and that have been treated with minimal to no post-processing. A second culture deals with higher levels of data fusion as classified in the Joint Directors of Laboratories (JDL) Data Fusion Group and Dasarathy models. The research presented in this thesis belongs to the latter community and is based on the use of the term "data" in its most general form as "information" that could be provided by sensors, humans, reports, etc.

In recent years, multisensor data fusion has attracted significant attention from researchers in a variety of fields. The main reasons for this popularity are the significant advantages that multisensor data fusion offers over single source data. The advantages that are relevant to the context of this research are as follows (Hall \& Llinas, 1997):

- Robust operational performance:

- Data contribution from one or more sensors, even when others are unavailable

- Increased probability of detection

- Extended spatial coverage:

- Ability of one sensor to detect a target that is hidden to other sensors

- Increased probability of detection

- Increased system reliability and confidence:

- Ability to confirm one event using different sensors

- Inherent redundancy that increases reliability and confidence

- Reduced ambiguity:

- Reduced number of hypotheses about the state of a target due to multisensory information

In the past, data fusion has been researched extensively for military applications. However, the advantages of multisensor data fusion listed above have motivated recent research in the AEC\&FM industry, including the present study. 


\subsubsection{Data Fusion Models}

The most complex and flexible fusion system architecture that serves as the rather elusive goal of researchers in a number of fields is the human brain (Dasarathy, 1997), which fuses signals from all five basic human senses at different levels and for different functions. Achieving the level of fusion complexity present in the human brain is only a dream at this point, but it describes the most ideal data fusion model. This section provides an overview of the most common data fusion models that are currently used by researchers in different fields. The fusion model developed in this research (Chapter 5) for an activity-based progress tracking system is based on the definitions of one of the existing fusion architectures.

Sensor fusion models are currently characterized based on the types of sensors used in the fusion model, the level of detail in the information, the application domain of the fusion, and the objective of the fusion model. Two main sensor fusion models are the basis of most other models: the JDL data fusion model and Dasarathy's model. This section describes these two models in detail, while noting the applicability of these and other fusion models in the context of the activity-based data fusion model presented in this thesis. The data fusion taxonomy explained in this section is referred to in the following chapters of this thesis.

\subsubsection{JDL Fusion Model}

The most popular data fusion model is the one established by the Joint Directors of Laboratories (JDL) Data Fusion Working Group, established in 1986 (Steinberg, Bowman, \& White, 1999). The JDL model, illustrated conceptually in Figure 2-1 (adapted from Haas, 2006; Steinberg et al., 1999), provides a practical and clear distinction between the different levels of data fusion. 


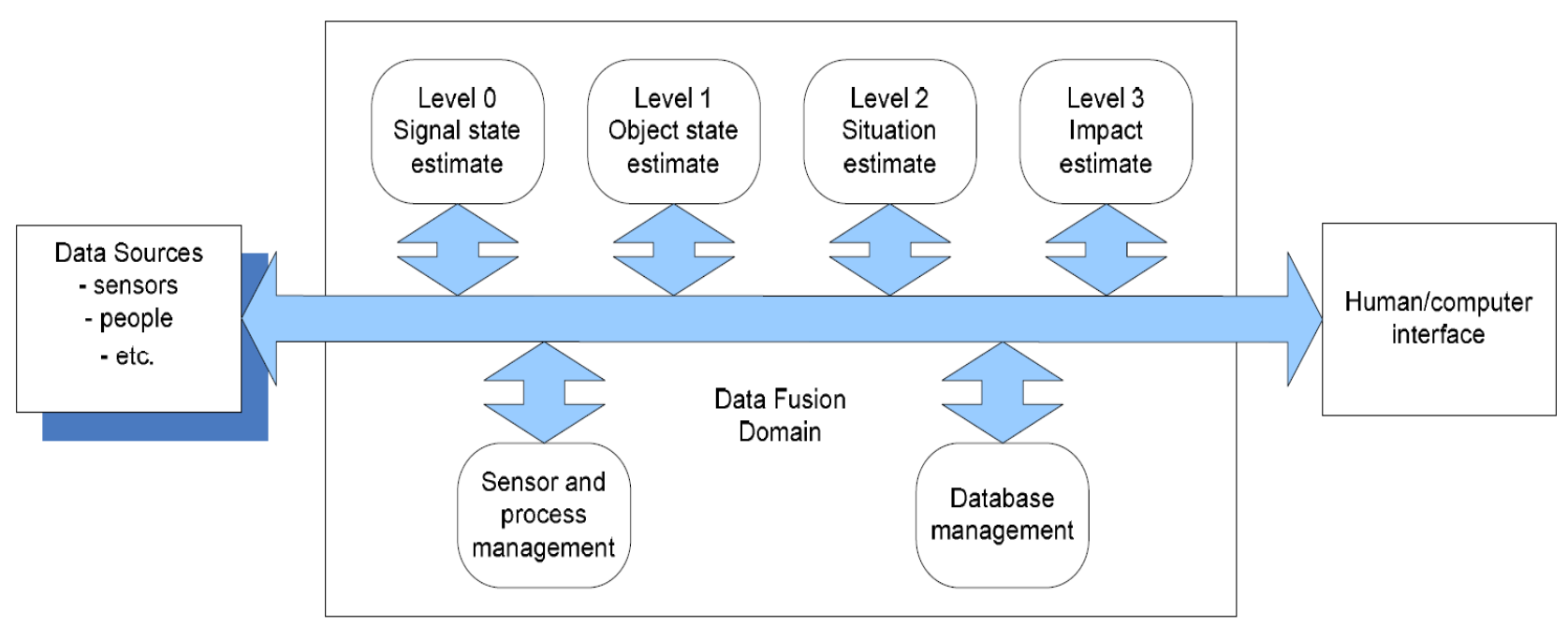

Figure 2-1: JDL Data Fusion Model Revised for Civil Engineering Applications

This model is explained in this section in the context of the AEC\&FM industry and, more specifically, with respect to tailoring it for the estimation of construction project progress. For automated construction progress tracking, the sources of information may include any combination of the following typical but not exhaustive list of sources:

- Radio Frequency Identification (RFID) tags

- Ultra-wideband (UWB) tags

- Object recognition from 3D laser scanners

- Schedule information

- Expert opinion, to be used in fuzzy logic memberships

- Project progress reports

- Payment and work-order files

As adapted from descriptions provided by Hall and Llinas (1997), the five levels of data fusion can be summarized as follows:

- Level 0 or source pre-processing: This level of data fusion is conducted at the signal level. Pre-processing can simplify or group the data so that the computational load at the higher levels of data fusion is reduced. 
- Level 1 processing or object assessment: At this level, data collected about objects from a variety of sources are combined to produce decisions about the state of an object.

- Level 2 processing or situation assessment: This level of data fusion is related to the relationships of the objects to other objects, and decisions regarding the relative situations of objects in the context of their environment can therefore be made at this level.

- Level 3 processing or impact assessment: This level of data fusion projects the impact of the current situation into the future, by providing a number of hypotheses about future events and an assessment of their impact.

- Level 4 processing or process assessment: The highest level of data fusion, this level includes processes that modify, control, or manage other processes.

Each level of data fusion processes a particular form of data and is used for a set of applications; therefore a multilevel data fusion model must process each level of fusion individually. Because the data fusion concept is applicable to numerous fields, the current published literature includes many other data fusion taxonomies, most of which represent only an extension of or a minor deviation from the JDL model presented in this section. Dasarathy (1997), however, presented a data fusion model that is fundamentally different from the JDL model, as explained in the next section.

\subsubsection{Dasarathy's Fusion Model}

Dasarathy's fusion model characterizes data fusion processes based on the input of the fusion model and the output generated by the model. More specifically, in this model, five data fusion processes are defined based on three levels of data hierarchy (data, feature, and decision) (Dasarathy, 1997).

Most other fusion models concentrate on either the input or output of the fusion process as a means of characterizing the fusion models, while in this model, the fusion processes are defined based on the relationships between the input and output variables. The levels of data fusion as defined in Dasarathy's fusion model are as follows: 
- Data in - data out (DAI-DAO) fusion: This level of data fusion corresponds to level 0 of data fusion in the JDL model and addresses the processes that are performed on the data in order to improve the quality or to modify it in any way. Any data filtering processes are included in this level.

- Data in - feature out (DAI-FEO) fusion: This level of Dasarathy's model is consistent with level 1 of data fusion and refers to the feature extraction processes in many data fusion models. Image processing techniques are an example of this type of data fusion, whereby features and objects are extracted from 2D images.

- Feature in - feature out (FEI-FEO) fusion: Dasarathy's model differs from the JDL model mainly with respect to this and subsequent levels of the model. Any fusion processes that improve the quality of information about an object or activity are included in this category of fusion.

- Feature in - decision out (FEI-DEO) fusion: This level of data fusion corresponds to the higher levels of data fusion in the JDL model. Studies of data fusion models for use in the construction industry have not explored this level of data fusion and have focussed primarily on the first two levels of data fusion, as characterized in this model. The main contributions of the data fusion model developed in the research presented in this thesis correspond to this and the next level of data fusion. At this level, decisions such as progress estimates are made based on the information provided at the feature level.

- Decision in - decision out (DEI-DEO) Fusion: This level is the highest level of data fusion as classified in Dasarathy's model. Any functionality that can help with the assessment of decisions could be classified to this level. The advantages and disadvantages of fusing data at different levels of the fusion model are explained in the next chapter. One of the objectives of this research was to leverage the advantages of high levels of data fusion for construction-related applications.

\subsection{Data Fusion Models in Construction}

This chapter has summarized the relevant research with respect to data collection on construction sites using state-of-the-art technologies as well as the existing data fusion models and taxonomies. This section is a summary of information about very recent data fusion models that have been developed in the construction research domain that synthesize the elements 
described in the preceding subsections for the purposes of automated construction progress tracking. The advantages and disadvantages of the existing models are also noted. Next chapter presents the conclusions with respect to the knowledge gaps that the research presented in this thesis has addressed.

In recent years, a number of researchers have considered earned value tracking as a more applicable and reliable means of tracking and estimating the progress of a construction project. Ghanem and Abdelrazig (2006) proposed a model based on wireless communication technologies in order to provide an estimate of the work completed on a construction project. In their research, RFID tags were placed on items on a site, and an earned value estimate was obtained using the tracking results from the RFID tags (Ghanem \& Abdelrazig, 2006). However, the problem with estimating earned value or progress estimates strictly from RFID tags is that the estimates obtained from these models are applicable only to those activities on a site that are directly associated with the movement of physical entities on a site, such as delivery activities. These models are incapable of dealing with many other activities on a site that are not directly associated with the movement of physical objects on a site, including some piping work, inspection, concrete curing, and interior finishing.

Kim et al. (2009) developed a system for the real-time progress management of steel structure construction projects by using an RFID system combined with a 4D model of the site. Their study was among the more sophisticated existing models that have been developed in an attempt to capture an accurate estimate of the progress at a site using material tracking technologies. They concluded that by using their proposed model, precise material quantities could be estimated and that material production, shopping, and onsite warehousing can be monitored in real-time (Kim et al., 2009). However, as with other studies that employed similar technologies, the most significant weakness of their proposed system remains the inability of the progress estimation model to measure other activities on construction projects. It should, however, be noted that for steel construction projects, the progress of the project is measured based on the number of tons of steel that have been installed. From this perspective, a materialbased or object-based progress estimate may be sufficient for use in the specific application of steel construction projects. Based on the same arguments, automated object-based recognition 
from 3D point clouds may also be sufficient for the progress tracking of steel construction projects (Bosche et al., 2008).

El-Omari and Moselhi (2011) suggested an integration model for the use of data acquisition technologies in progress reporting of construction projects. In their proposed model, data from barcoding, RFID, 3D laser scanning, photogrammetry, multimedia, and pen-based computers would potentially be integrated. Ideally, a user could move around the construction site with a tablet PC and record the conditions at the site using a variety of data collection modes, such as handwritten notes, snapshots, or even voice comments (El-Omari \& Moselhi, 2011). As with many other researchers working in the area of construction progress tracking, El-Omari and Moselhi also attempted to track the "earned value" of the construction project rather than what is usually referred to as progress tracking, which is consistent with industry demands. They also suggested an interesting platform for the fusion of a variety of sources of information on a construction site (El-Omari \& Moselhi, 2009b). Although their published research did not present the fusion algorithms or strategies that they propose to employ, their goal of integrating a variety of sources of information on construction projects is in line with the efforts of many other researchers in the field of automated construction progress tracking.

Golparvar-Fard et al. (2009) also recognized the inefficiencies of existing progress reporting methods, such as progress S curves, schedule bar charts, and photographs and textual reports, in presenting and visualizing multivariable site information (including schedule, cost, and performance). They pointed out that existing progress tracking systems can be categorized into two main systems: monitoring physical progress through either percentile or budget-based monitoring, both of which are complex reporting systems and are rather subjective with respect to the way in which the percentages or budget amounts are calculated and reported (GolparvarFard, Pea-Mora, Arboleda, \& Lee, 2009a). They therefore proposed a visualization system for monitoring construction progress using 4D simulation models overlaid on time-lapsed photographs. In their proposed visualization system, 4D simulation of the CAD model was used as the as-planned progress information, time-lapse photography and videotaping were used as the as-built progress data, and integrated visualization was achieved by augmenting the as-built photograph with the as-planned data. Integration of these sources of information allowed deviations from the as-planned model to be identified. They also integrated the schedule 
information with the information about the progress at the site, based on which deviations from the scheduled plan were also calculated.

In another study, Golparvar-Fard et al. (2009b) investigated the use of unsorted daily progress photographs for construction progress estimation. The motivation for their research was the fact that photography logs are usually available from many construction projects and can be easily captured using very low-cost data collection techniques (Golparvar-Fard, Feniosky, \& Savarese, 2009b). The photographer's locations and orientations as well as a sparse 3D geometric representation of the as-built site were obtained from the photographs, which were then compared with the as-planned 4D model in order to estimate the progress of the construction site.

It should be noted that, as with most other fusion models presented in the literature, the preceding object-based progress estimation methods would work well only for assessing the progress of steel structures, where the progress is usually reported in terms of the number of tons of steel installed. These fusion algorithms are not able to deal with a large number of other activities on a construction site, including many piping activities, which could account for up to $50 \%$ or more of a project's value, particularly in the case of industrial projects. Piping activities include welding and inspection which are not directly associated with the presence and movement of objects on a site. This shortcoming of the existing data fusion models in dealing with the variety of activities on construction sites was the primary motivation behind the first two objectives of the current research.

In addition to the studies of data fusion that have focussed on automated construction progress tracking, recent research related to data fusion models has explored their use for other management support systems. Pradhan et al. (2011) presented an innovative query-based data processing model to support data fusion for construction productivity monitoring. In the proposed query-based system, a computer-interpretable query capture language was developed in order to capture dynamic user queries and to process data to produce the information required (Pradhan, Akinci, \& Haas, 2011). Although no attempt was made to use this model to fuse data for the purposes of automated construction progress tracking, its innovative and interesting query-based approach could be used for reporting the results of a variety of other data fusion models, including the model presented in this thesis. 


\subsection{Data Management}

Most researchers working in the areas of automated data collection and data fusion in the construction domain acknowledge the increasing problem of storing and managing the data acquired from construction projects. As different technologies are being employed in construction projects, an enormous amount of data is being collected automatically or semiautomatically, with a resultant strong demand for a reliable data management system. This situation is not unique to construction projects, because other sectors of the civil engineering domain, such as water distribution networks, are also equipped with a variety of sensors and data acquisition systems that generate a wide variety of data for the support of data fusion and real-time decision making (Joseph, Adams, \& McCabe, 2010). A number of data management systems have been proposed for use in the civil engineering domain as a means of dealing with the exponentially growing amount of research data. For example, El-Omar and Moselhi (2010) presented a data management framework for data collected using a variety of data acquisition technologies. Their model was developed as a way of organizing, storing, retrieving, and processing the data collected for project control purposes (El-Omar \& Moselhi, 2010). However, most of the existing data management efforts are very specific to an individual project and are not scalable to the construction research community. They also lack many of the attributes necessary for an effective and reliable data sharing and management system, as explained in Chapter 6.

In addition to the data management issues with respect to the large amount of data being collected on construction projects, document management on construction sites has also become very challenging with the increasing number and types of documents to be managed, particularly in mega projects. Shehab et al. (2009) also identified document management for construction projects as a significant risk factor with regard to project execution. They proposed a very simple barcode-assisted data acquisition method for managing the documents related to construction projects: a barcode tag was attached to each document and tracked by scanning the barcode. Their proposed system was reportedly nine times faster than standard manual data entry and saved up to $39 \%$ of the total time required for document management (Shehab, Moselhi, \& Nasr, 2009). 
Recent advances in construction research are driving the construction industry toward increased connectivity and communication; building information modeling and the evolution of the integrated design approach are examples of how information from different sources is being combined and transferred in order to improve productivity and reduce risk in construction. However, the studies behind these advances are typically not the product of a collaborative and a communicative research network, and no effective universal system for sharing the raw data obtained from construction research endeavours currently exists.

The drive toward connectivity and communication is also evident from a more global perspective. Research funding agencies, such as the US National Institute of Health $(\mathrm{NIH})$, the National Science Foundation (NSF), and the National Institute of Justice (NIJ), have recently begun to require that all research data produced through their funding be shared with other researchers. These research funding agencies have recognized the importance of research data sharing for the progression of knowledge. As a result, a need has arisen for an effective and efficient means of managing and sharing research data and a sophisticated communication and process management system for collaborative research.

The NIH has implemented a policy that requires researchers who are applying for large amounts of funding (more than $\$ 500,000$ per year) to include in their applications a data-sharing plan (National Institutes of Health $(\mathrm{NIH}), 2003)$. The NIH considers all data suitable for sharing as long as the privacy of the participants is protected and does not specify a method for data sharing but instead leaves these details to the researchers and their communities.

The NSF requires that proposals specify a data management and sharing plan that includes the dissemination of research results (National Science Foundation, 2011). The data management plan is considered a necessary part of the proposal and is a component included in the evaluation of the application for funding. This system acts as an incentive for researchers to complete well-thought-out and even innovative data management and sharing plans. The NSF requires that researchers share their primary data within a reasonable time frame but recognizes the immediate cost implications of preparing the data for dissemination.

The NIJ requires that applications for funding include a data archiving strategy and a data dissemination strategy (National Institute of Justice, 2010). As with the NSF data management 
and sharing plan, the NIJ evaluates the data archiving and data dissemination strategies proposed by applicants on the basis of intellectual merit and the broader impact of the research.

\subsubsection{Advantages of Sharing Research Data}

The reasoning of agencies such as the $\mathrm{NIH}, \mathrm{NSF}$, and NIJ with respect to the promotion of the sharing of research data is also applicable to the construction research community. The advantages of sharing research data are numerous, the most significant of which is the progress of science. Sharing research data allows researchers to work together and to build on one another's work rather than repeating the same research (Fischer \& Zigmond, 2010). In this way, research communities can act as a whole to accomplish greater and larger-scale research endeavours. The best way to explain the advantages of research data sharing is to compare collaborative research activities to the recent growth in joint ventures in the construction industry. Mclntosh and McCabe (2003) investigated the advantages of international construction-consulting joint ventures in the English-speaking Caribbean. They concluded that the advantages of such joint ventures included the opportunity to enter new markets, the sharing of risks, access to new technologies, and increased ability to obtain new work (Mclntosh \& McCabe, 2003). The same advantages can apply to the construction research community because, researchers too, would be able to enter new markets, share the risks or costs associated with their research projects, gain access to new technologies that they would not otherwise be able to afford, and obtain new sources of data that would lead to new research projects.

From the perspective of the research funding agencies, sharing research data is beneficial because it allows many researchers to access and use the same data set, thereby maximizing the use of the data (Axelsson \& Schroeder, 2009). As a result, research funding agencies receive better value for their funding investments when any data set can be accessed and used for multiple research interests. Additionally, individual researchers have increased opportunities to contribute to the research community without the burden of research collection costs (Fischer \& Zigmond, 2010). In many research projects, including the current research documented in this thesis, a large quantity of data are collected but are rarely fully utilized. The data are often very costly to obtain, both financially and in terms of manpower, in which case not many research programs may be able to afford to acquire them. Logistics and geographical distances also 
prevent some research groups from obtaining the data they require. For example, in an effort to evaluate the impact of the redevelopment and expansion of airport operations, one research project from the University of Toronto collected data from 22 North American airports, 26 airport projects, and 107 individual operation years (Hantziagelis \& McCabe, 2006). Other researchers in East Asia or even Europe would not normally be expected to have access to this amount or level of data from North America. Likewise, if such data were collected from European or Asian countries and then shared with the entire community, more accurate and applicable conclusions could be obtained, resulting in a more significant impact on the industry.

Open access to research data promotes problem solving from diverse perspectives, which ensures a holistic approach that benefits all stakeholders in the research as well as the research area itself. Researchers can also validate each other's work through data sharing, so that the increased transparency of research can help to ensure its quality (Fischer \& Zigmond, 2010; Weil \& Hollander, 1990). Open access to research data also allows its use by researchers who may not have sufficient resources to produce original research data (Fischer \& Zigmond, 2010). Reducing the burden of data collection costs effectively promotes research and thus helps to further the development of research.

In response to the requirements set out by research funding agencies, some research institutions (namely universities) are beginning to develop their own guidelines for sharing research data. For example, the Heriot-Watt University of Edinburgh requires that, after research has been published, the research data be made available to others upon request (McFadzean, 2003). The University of Pittsburgh has also developed research-sharing guidelines, which identify the researcher as the party responsible for recording and sharing the research data and require that all research data developed with federal funds (particularly those from the NIH and NSF) be shared with other researchers upon request (University of Pittsburgh, 2009).

\subsubsection{Challenges with Research Data Sharing}

A number of challenges are associated with the sharing of research data. The central issue is the lack of widely accepted data-sharing mechanisms, including the associated technical difficulties, such as digitizing, formatting, organizing, maintaining, documenting, protecting, and 
providing access to the data (Axelsson \& Schroeder, 2009; Giffels, Vollmer, \& Bird, 2010; Weil \& Hollander, 1990). All of these technical data management issues require funds, time, and personnel for their resolution, thus providing disincentives that inhibit sharing. Since the responsibility to share data falls on the researcher, it is the researcher who must invest effort and funds in the necessary data management.

The lack of incentives for researchers to share their raw data goes beyond technical issues. There is a negative perception of data sharing among researchers because sharing data, while furthering the progress of science in general, can also be seen as providing an advantage to other researchers who are seen as competitors within the research community (Ceci, 1988). Consequently, researchers are actually provided with incentives not to share their research data so that they will be guaranteed the maximum reward for their work in the form of additional funding, exclusivity, multiple publications, and prestige.

No recognition system exists for research data sharing that is comparable to the recognition associated with article citations, and data sharing is not considered as a qualifier for further research funding as published articles are. By providing no recognition or compensation for sharing research data, the current system in fact supplies incentives not to produce original data but to use others' research data instead (Fischer \& Zigmond, 2010). This situation defeats the purpose of sharing data because, if no new research data were collected, progress would lose momentum.

Finally, the research by McIntosh and McCabe (2003) related to the risks and benefits of international construction-consulting joint ventures also identified risks associated with collaborative work, including project delay and higher levels of bureaucracy for many administrative tasks such as approvals. These risks could arguably be applicable to research data sharing and consequently to joint-venture research projects. Therefore, the goal of the developed data-sharing and management system of the current research was to address all of the disadvantages and challenges that have been identified in this section so that the system might be accepted as a viable solution by the construction research community and might encourage research data sharing and management. 


\subsubsection{Existing Data Sharing Models}

A need has arisen within the construction industry for an effective and efficient means of managing and sharing data that takes full advantage of the benefits of data sharing. As evidenced in the current research, an enormous amount of data are being collected for a variety of projects and are not being fully utilized due to the lack of an effective data management or data sharing system. To date, few widely used data sharing models have been developed. In this section, the two systems most commonly used are described: web-based collaboration portals and web-based SharePoint. The data sharing model developed through the research for this thesis is presented in Chapter 6.

\subsubsection{Web-Based Collaboration Portals}

Collaboration portals facilitate the sharing of information and data by providing interested parties with a single point of access that enables them to interact with communities of practice. Portals of varying complexity and design have been established in virtually every industry genre, both on the World Wide Web and for individual consortiums such as the Construction Industry Institute. Types of portals differ to a large extent with respect to the objectives, scope, financial investment allocated, and technical limitations. A portal can be as simple as a wiki-based system with no database backend, wherein the primary focus is on the creation and dissemination of documentation, or it can be as complex as a semantically rich repository of information and data that augments the creation and transfer of flow of information within a community. Several recent semantic-driven portals based on the creation of ontological taxonomies have been proposed, even within the construction research domain (El-Diraby, 2010; El-Gohary \& El-Diraby, 2010), but in practice, few have been implemented.

A variety of academic communities have created collaboration portals for virtually every sphere of focussed research. Informal collaboration portals are often fragmented and are usually the result of a few universities developing joint initiatives for a particular project. In general, these types of portals are quickly set up, but due to the short lifespan of the projects and funding constraints, the inability to maintain such portals for a significant length of time inevitably leads to their abandonment. Structured portals that receive industry or alumni-assisted support have longer shelf lives but require dedicated personnel for the maintenance of the system. For 
example, several university libraries, such as the University of New York's Library, use wikibased systems to foster information sharing among librarians (Grace, 2009).

In this section, the system used as an example of a web-based collaboration model is Wikipedia, which is an evolution of Nupedia. The Nupedia vision was to provide a low-cost, online encyclopaedia (Reagle, 2009). However, Nupedia's progress was slow because it relied on expert volunteers for both the writing and the editing of its articles. To increase productivity and to promote interest in the online encyclopaedia, Nupedia evolved into Wikipedia through two fundamental changes. First, both the browsing and the editing processes were performed online and, second, anyone with access to the internet was now permitted to contribute to the encyclopaedia.

Wiki-based collaboration systems allow anyone to instantly access, create, edit, and delete any information at any time via the internet (Yang \& Lai, 2011). The fact that the system stores information regardless of its source is both its strength and its weakness. The open and collaborative environment this system promotes builds a comprehensive body of information but also entails quality control and vandalism problems.

A wiki-based system for sharing data is easy to access and use because its interface is familiar and open to anyone who has access to the internet. The information in the system is provided free of charge, and both search results and publications are available instantaneously, making it an attractive source for users and contributors alike (Tseng \& Huang, 2011; Yang \& Lai, 2010). Contributing to a wiki-based cloud is also a simple process requiring little programming knowledge. Accessibility promotes collaboration within the cloud and reduces barriers to data sharing. These characteristics of simplicity and accessibility are crucial for a construction research data management system because widespread collaboration is the ultimate goal of the system.

Wiki-based cloud systems rely on the collective discernment of contributors to ensure the accuracy of the information in the cloud (Yang \& Lai, 2011). However, since any information can be changed at any time, there is no way of ensuring that the information being received at any specific time is correct. Contributors may be vandals, activists, speculators, partially informed, or misinformed (Denning, Horning, Parnas, \& Weinstein, 2005). There is no 
mechanism for validating the credentials, motivations, or sources of a content contributor. The only technique available in a wiki-based cloud system for mitigating the effects of the entry of inaccurate information is the recording of content revisions for recovery (Tseng \& Huang, 2011). The accuracy of the information available in a research data management system must be high so that the information can be used to further scientific progress. The minimal quality control associated with a wiki-based cloud may not be strict enough.

A web-based cloud also has no contributor recognition system. Although the content can be tied back to the contributor, no systems or methodologies are in place for the preservation of intellectual property. Therefore, when they share information, users lose ownership and the benefits associated with any new information added to the cloud (Yang \& Lai, 2010; Yang \& Lai, 2011). The time and effort that information sharing requires is the responsibility of the contributors, but there is no recognition or compensation awarded to contributors for sharing information (Yang \& Lai, 2010). It is therefore difficult to imagine construction researchers or other researchers sharing their raw data through a web-based collaboration portal system.

Wiki-based systems are often used to facilitate the documentation of project modules by the experts who were involved in their development. Their primary purpose is therefore the creation and dissemination of information as well as potential collaboration. These objectives are often achieved by the creation of simple html pages and version control software, which can be modified on request by a user, but there is no underlying data structure for managing large volumes of associated data. A construction research data sharing model that is based on a wiki driven system would require substantial modification to the wiki engine to enable data storage and retrieval. A dedicated back-end database would have to be designed and maintained in order to accommodate the wide range of data and information about the associated projects for every specific research initiative. The scalability requirements of such a system would make this choice impractical.

\subsubsection{Web-Based SharePoint}

Another existing data management and sharing model is the web-based SharePoint system, which can be accessed by members who can view aggregated data and enter their own. For the purposes of this research, the Construction Industry Institute (CII) Benchmarking and 
Metrics Program is used as an example of a web-based SharePoint site. CII is a pool of public and private engineering firms that collectively seek to improve the construction industry by sharing information. Cll's purpose is to measurably improve performance in the construction industry through the collaborative efforts of industry professionals and academics (Construction Industry Institute, 2009). The CII Benchmarking and Metrics Program is a tool that CII member companies can use to compare their construction projects to a database of metrics of completed projects. The metrics include cost, schedule, safety, changes, and productivity and can be used to plan and measure project performance relative to that of similar projects in the database (Lee, Thomas, \& Tucker, 2005). Cll member companies can also contribute to the database by reporting their own project experiences.

The web-based SharePoint system, much like the wiki-based cloud system, is accessible via the internet. However, access to the database of a web-based SharePoint system is restricted to members. These members must pay dues and be actively involved in research activities. These accessibility restrictions impose resource constraints and thereby limit the diversity of contributors to such a site. For example, small businesses may not be able to participate in the research work due to the lack of funds and participation resources required for membership. Entire sectors of project information could be missed that could be used for a comparison of metrics and for research. If construction research data were managed through a web-based SharePoint system, the lack of contributor diversity could hinder the progress of construction research. However, this limitation alone is not sufficient reason for dismissing the SharePoint alternative, because any feasible solution would have a contributor restriction to ensure quality control as well as to address scalability issues.

The web-based SharePoint system can be used for the collection and automatic aggregation of data for publication. Data is continually being collected and can be submitted at any time by contributors via the internet (Lee et al., 2005). Since the SharePoint site has paying members, the quality of the content is critical to its success, requiring control mechanisms to be in place. To ensure that high-quality information is being disseminated to its members, data entry tools employ algorithms that identify inconsistencies in the data being entered (Lee et al., 2005). As just one example of a quality control mechanism, when an inconsistency is identified, the user can be notified and asked to make a change. Another method of quality control is contributor 
control, which can be accomplished through member requirements. For example, Cll members must be part of the construction industry or academia, must pay dues, and must participate in research-related activities. These requirements validate the motives and credibility of collaborators. Such validation makes the SharePoint system generally more reliable than the cloud system and therefore perhaps more appropriate for research data management.

A web-based SharePoint database can also be expanded through the requirements for member participation. To enable them to benefit by comparing their work to that of other members, members must input data from a sample of their projects (Construction Industry Institute, 2011). These types of requirements might be used to build a widespread construction research data management system to ensure that researchers continue to collect their own original data that will contribute to the overall research effort. However, the quantity of data on a SharePoint site is also somewhat limited by the access-restricting membership requirements. In addition, significant increases in the volume of data to be stored can seriously and detrimentally affect the performance of a web-based SharePoint system. To overcome such hindrances to effective performance, expensive hardware resources may be required on an ongoing basis to facilitate the inclusion of a high volume of construction research data projects.

Although a web-based SharePoint system has no recognition mechanisms in place for individual contributions, other forms of compensation for members are made possible through membership fees and research participation. For example, Cll offers its members free access to all of its benchmarking and metrics reports, to education and training resources, to networking opportunities, and to member-only events and products (Construction Industry Institute, 2010). A similar compensation system could be applied to a construction research data management system to create participation incentives, provided members of the construction research data management system are willing and able to pay membership fees.

The research for this thesis also resulted in the identification of the data and document management for construction projects as a significant limitation with respect to a number of fusion mechanisms and other managerial applications, including earned value estimation, productivity analysis, and prompt schedule updating. One of the goals of this research was therefore to develop a reliable, efficient, and effective data management system for construction research data. Chapter 6 of this thesis deals specifically with this objective and presents the 
proposed data management system developed through this research, which goes well beyond the scope and depth of existing data management systems in use in the construction research community.

\subsection{Summary}

This chapter has summarized the relevant research with respect to automated construction progress tracking, applicable sensory sources of information, and multi-sensor data fusion models. The automated and semi-automated data collection techniques including bar coding systems, RFID systems, GPS, UWB positioning systems, and BIMs were investigated. The two most commonly used data fusion models were also presented: JDL and Dasarathy data fusion models, followed by a summary of recent data fusion models that have been developed in the construction research domain for the purposes of automated construction progress tracking. It is concluded that the five levels of data fusion as defined by the JDL model are more compatible with the fusion processes of this research and therefore the JDL model has been adopted for defining the various fusion aspects of this research. Finally, a review of the existing data management models was presented, which indicated a need for an efficient, reliable and scalable system for the management and sharing of construction research data. The next chapter presents the knowledge gaps evident from the literature as well as the research vision, approach, and structure. 


\section{Research Vision}

The vision for the research presented in this thesis was developed based on the knowledge gaps that were identified from the published literature. A review of these knowledge gaps is presented in this chapter, followed by the research approach that was followed. This chapter concludes with the structure of the research that is presented in following chapters of this thesis.

\subsection{Knowledge Gaps from Literature Review}

The sensor-level processing stage, or the object-assessment level, of data fusion has dominated the majority of research activities related to multisensor data fusion for construction industry applications. In many applications, such as in automated progress estimation, an object-based data fusion process is insufficient because many construction activities are not directly associated with a measurable physical entity on the site and therefore cannot be identified using traditional sensing techniques. These activities include but are not limited to inspections, installations, welding, and interior finishes.

A review of the literature related to existing fusion models further revealed that despite the advantages of the Dasarathy's model for workflow based processes, the data fusion algorithms and engines required for this research were more compatible with the definitions within the JDL model. This research acknowledges the complementary advantages of these two data fusion models, however, from a functionality perspective, the classification model would not have a significant impact on the development of the fusion model. In order to maintain a consistent terminology throughout this thesis and also to maintain consistency with the existing literature in the data fusion domain, the fusion level definitions from the JDL model were adopted for the development of the fusion model presented in this research. The JDL model proved to be a compatible model for the data fusion and management processes defined in this research.

A review of existing data management models, including web-based cloud and web-based SharePoint models also revealed the current lack of an effective universal and comprehensive system for either managing data required for fusion or for sharing the data obtained from construction research endeavours. The wiki-based cloud and web-based SharePoint systems each have features that would be desirable in a construction research data management 
system. However, these systems also each have limitations that limit their suitability for construction research data management and dissemination.

The next section presents the research approach and the model that encompasses all elements of the research presented in this thesis and which addresses the knowledge gaps with respect to the lack of a consistent and reliable data fusion and management system.

\subsection{Research Approach}

This section presents the workflow based approach of this research, followed by the data ontology that is used throughout this thesis. A brief evaluation of data sources available on construction projects is presented next, followed by the developed data fusion and management architecture, presented within the JDL framework.

\subsubsection{Workflow Driven Design}

In this research, a workflow based data fusion and management framework is developed for construction progress, quality and productivity assessment. Workflow is a term used to describe, execute and control the sequence of tasks in a business process, including procedural steps, people or stakeholders involved, as well as the input and output that is required. Data fusion and management process workflow implementation via a distributed computing network and archiving using a cloud-based architecture are both illustrated in this research.

In the context of the current research, a workflow refers specifically to the process defined for the flow of information and tasks through a system. The Workflow Management Coalition [WfMC] Specification, which was founded in 1993 to establish workflow standards based on processes, defines a workflow as "the computerized facilitation or automation of a business process, in whole or part" and further notes that workflows are "concerned with the automation of procedures where documents, information or tasks are passed between participants according to a defined set of rules to achieve, or contribute to, an overall business goal." As such, workflows promote the process-oriented modeling of activities. In recent years, workflows have been implemented for a wide range of applications from formalized data processing activities for extracting bridge components from 3D laser point clouds (Tang \& Akinci, 2011) to a 
workflow-driven approach for the energy performance retrofitting of existing buildings (Larsen, Lattke, Ott, \& Winter, 2011).

In this research, a workflow based approach is combined with traditional object-based logic and programming. The workflow management focuses on the processes within the model, while the object-based components focus on the collected data and construction documents. Chapters 5 and 6 of this thesis present the developed workflow based data fusion and data management systems of this research, respectively.

\subsubsection{Data Ontology}

For this research, the following data ontology was used, which categorizes all available sources of information into four main categories based on the type of information obtained from each source:

- Volumetric: the data sources in this category provide information for volumetric progress measures of a site. Object recognition results from point clouds obtained from either 3D laser scans or photogrammetric techniques are included in this category.

- Positioning: all data sources that can be used to indicate an object's location in a global co-ordinate system, a local co-ordinate system, or even in a proximity system, are categorized as positioning data sources. These sources include Global Positioning System (GPS), Ultra Wide Band (UWB) positioning system, and RFID systems.

- Project control: all other sources of information that can be obtained from a construction project that are not directly associated with individual objects, but are specific to a construction project, such as the schedule, payment reports, earned value reports, etc. are included in project control category.

- Generic information: the generic sources of data included the sources that provide information that is not specific to a construction site. For example, weather information was categorized as generic information source, since the information it provided was not specific to a given construction site. The fusion of generic sources of data was outside the scope of this research. 


\subsubsection{Data Source Evaluation}

The scope of the fusion model development and field implementation for this research was limited to the piping activities of a building construction project, which entails five categories of associated activities: delivery to laydown yard, delivery to the installation site, installation, welding and/or fitting, and inspection. The relative contributions of these activity categories are shown in Figure 3-1. These percentages were defined based on the expert opinion of the project manager of the site where the data fusion model was implemented.

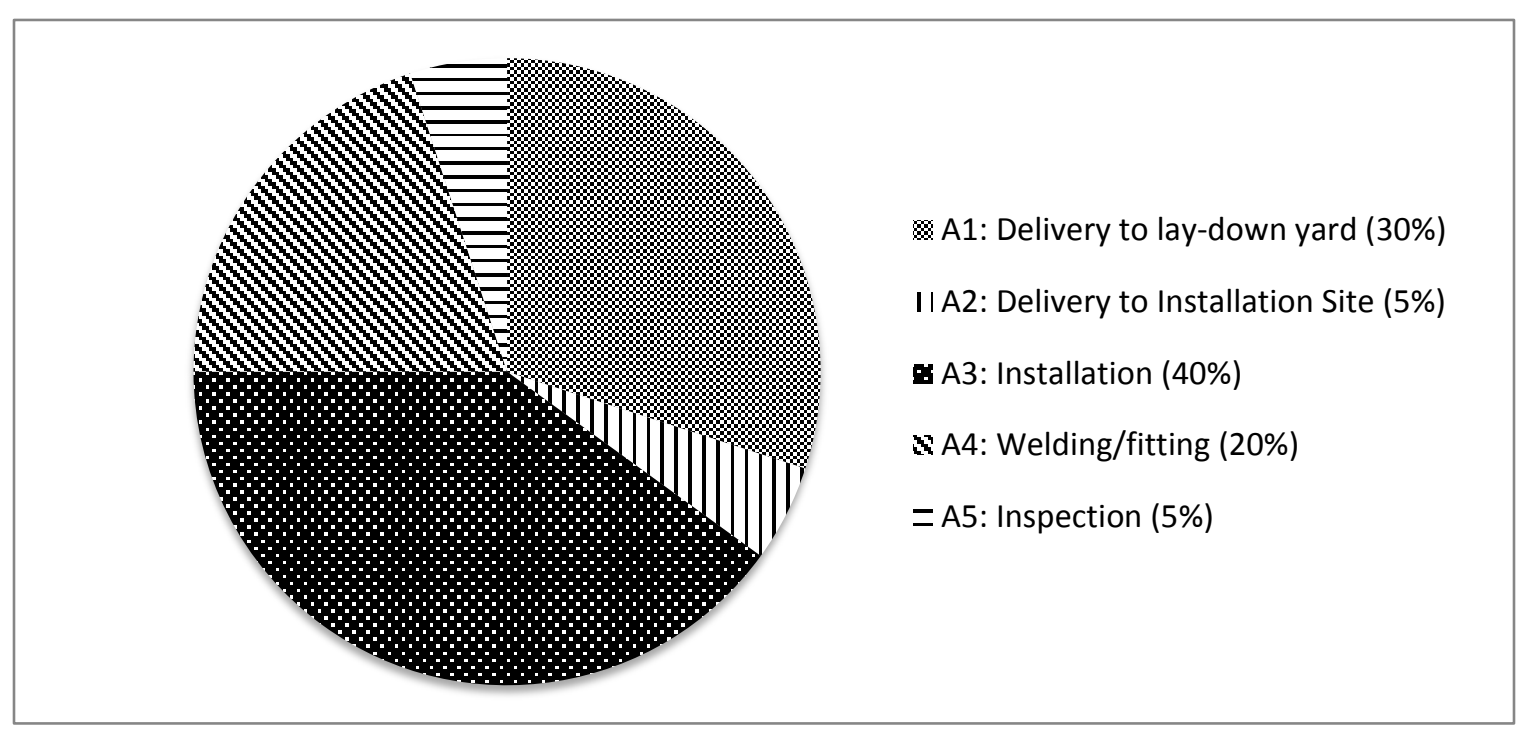

Figure 3-1: Value Breakdown of E6 Piping Project

Since the relative contributions of these activity categories may change from project to project, the percentages shown in Figure 3-1 were used as one possible state of one of the inputs into the model, and they can be easily modified. The number of categories can also be adjusted through minimal design changes to the fusion architecture of the model developed through this research.

A number of sources of data could be used to measure the progress of each of these activity categories. Table 3-1 summarizes the sources that could potentially be used for assessing the progress of each activity category. In this table, "Yes" refers to a situation in which a particular technology or source can readily provide information regarding the progress of an activity group, "Indirect" refers to situations in which either the information is unreliable or algorithms are 
required for progress decisions, and "No" refers to situations in which the type of information required cannot be extracted due to the characteristics and limitations of the data source. For example, UWB and RFID tags can be used to indicate whether a target has been delivered to the site and/or installed. However, they cannot automatically indicate the difference between a piece of pipe that has been installed and one that has been welded or inspected.

Table 3-1: Potential Sources of Information

\begin{tabular}{cccccc}
\hline Data Source & $\begin{array}{c}\text { Delivery to } \\
\text { Laydown yard }\end{array}$ & $\begin{array}{c}\text { Delivery to } \\
\text { Installation Site }\end{array}$ & $\begin{array}{c}\text { Welding } \\
\text { Installation } \\
\text { and/or } \\
\text { Fitting }\end{array}$ & Inspection \\
\hline BIM & No & Indirect & Indirect & Indirect & Indirect \\
GPS & Yes & Yes/No & Yes/No & No & No \\
UWB & Yes & Yes & Indirect & Indirect & Indirect \\
RFID & Yes & Yes & Indirect & Indirect & Indirect \\
Photogrammetry & Yes & Yes & Yes & No & No \\
3D Scan Data & Yes & Yes & Yes & No & No \\
Schedule & Yes & Yes & Yes & Yes & Yes \\
Inspection & No & No & No & Yes & Yes \\
Report & Indirect & Indirect & Indirect & Indirect & Indirect \\
Progress Report & Indirect & Indirect & Indirect & Indirect & Indirect \\
\hline
\end{tabular}

To provide an efficient model for an automatic construction progress estimation process, reliable and accurate sources of data are required. Data sources for the developed model of this research were chosen based on the performance of the technologies as reported in the published literature, the cost and accuracy of the available technologies, and the data requirements of the developed fusion algorithms. The previous chapter summarized an extensive review of the state-of-art technologies for data collection on construction projects. The review of the literature revealed that an objective evaluation of the photogrammetry and 3D laser scanning technologies was required in order to facilitate the choice of the most suitable solution for point cloud acquisition, which is presented in section 4.1 of this thesis. Point clouds 
were used for automated object recognition, which was one form of input into the fusion model developed through this research.

The review of the published literature further revealed that combined RFID and GPS technology is not feasible for indoor positioning and other RFID related technologies do not provide the level of accuracy needed for the fusion algorithms of this research. Therefore, it is further concluded that UWB technology would be the best fit for location estimation as well as for material and Activity Progress Tracking applications for indoor construction environments. However, a detailed investigation and analysis of its performance in construction environments was required before its data could be implemented in the fusion processes required for this research. The results of this investigation and analysis are presented in section 4.2 of this thesis.

\subsubsection{Data Fusion and Management Architecture}

The workflow driven data fusion model that was developed in order to fuse the information from the various data sources is shown schematically in Figure 3-2, and presented in detail in Chapter 5 of this thesis.

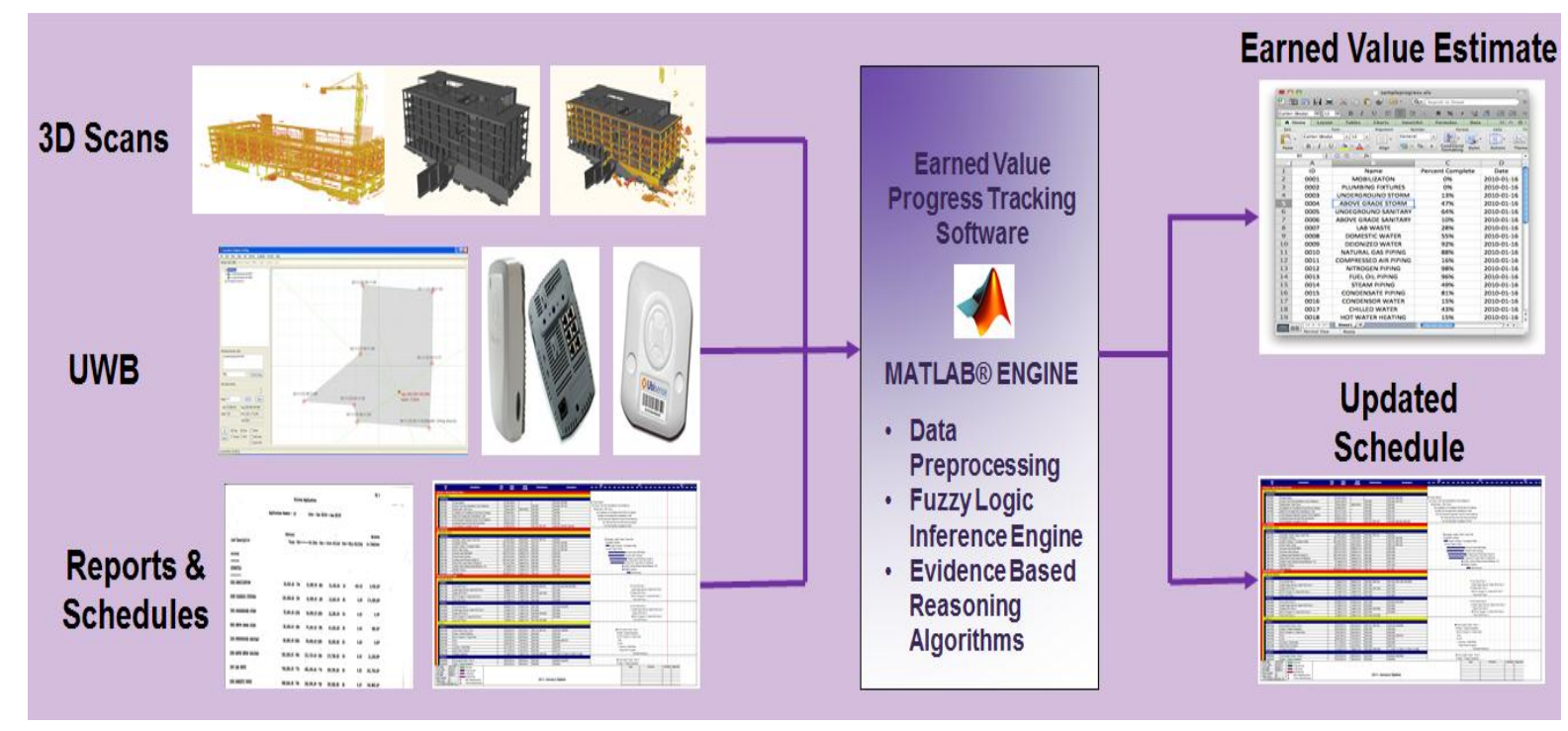

Figure 3-2: Schematic Representation of the Developed Fusion Model 
The input sources were fused in a MATLAB environment with the use of a number of fusion strategies, as explained in Chapter 5. The JDL model, shown conceptually in Figure 3-3, provides clear and useful distinctions between the different levels of data fusion, and fusion processes in the MATLAB engine are therefore defined in this section in terms of the fusion level definitions used in the JDL data fusion model.

The five levels of the data fusion processes used in the current research can be summarized as follows:

- Level 0, or source pre-processing: This level of data fusion is conducted at the signal level. Pre-processing can simplify or group the data so that the computational load at the higher levels of data fusion is reduced. In UWB positioning technology, data filtering can be classified as source pre-processing. In the context of this research, the location estimation algorithms of the UWB technology are classified as level 0 data fusion because they fuse data from different receivers in order to estimate the location of the tag, which is then communicated to the data acquisition system.

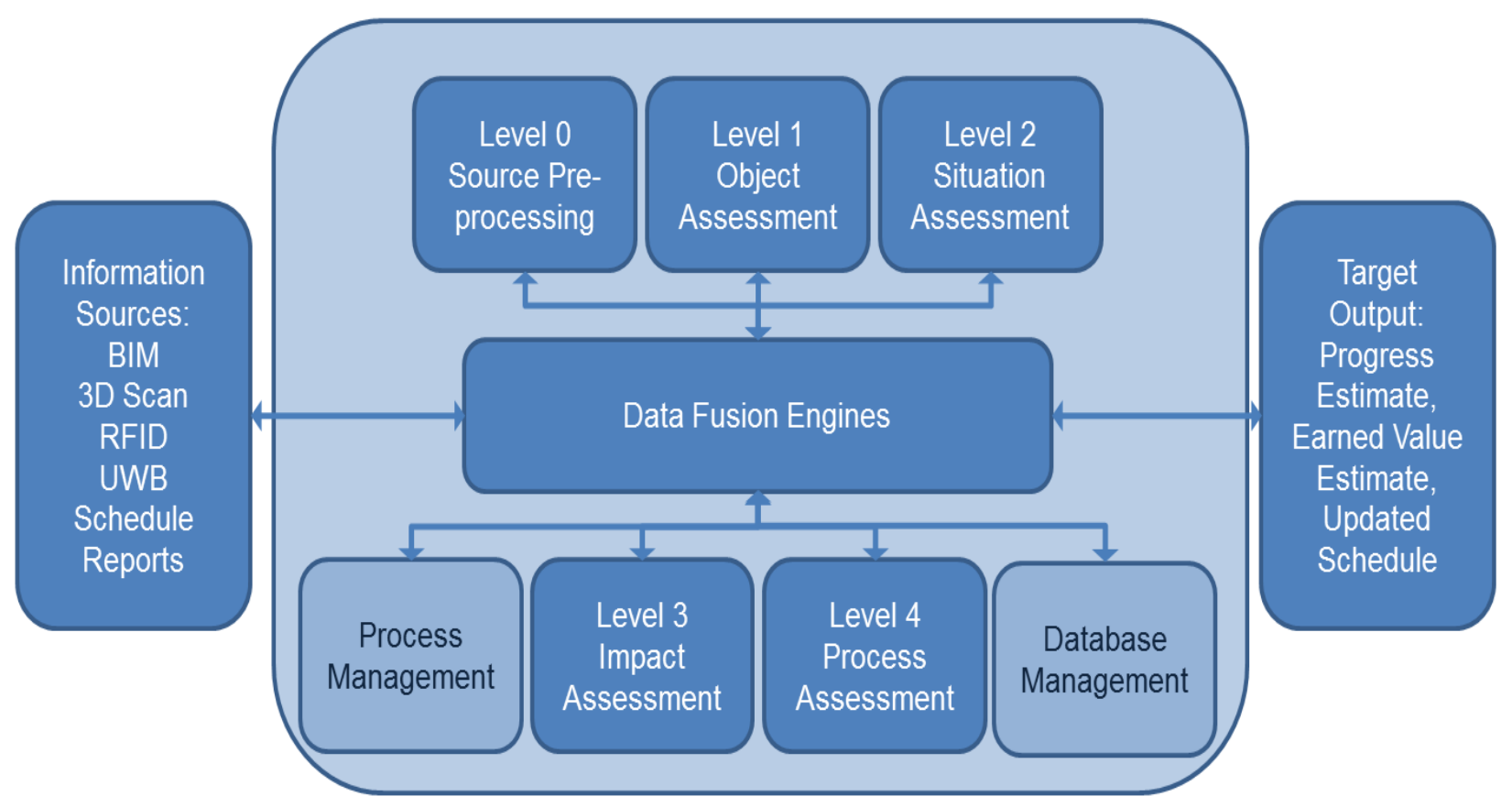

Figure 3-3: Revised JDL Data Fusion for Construction Progress Tracking 
- Level 1 processing, or object assessment: At this level, the data collected about objects from a variety of sources are combined to produce decisions about the state of an object. In the current research, with the use of other available sources of information, such as UWB readings, logical algorithms are employed at this level of data fusion as a complement to the object recognition results from 3D scans.

- Level 2 processing, or situation assessment: This level of data fusion is related to the relationships between the objects, and decisions regarding the relative location of the objects in the context of their environment can therefore be obtained at this level. In the fusion model used in this research, the initial interaction between the activity-based fusion and the object-based fusion takes place at this level. This level of data fusion also forms the basis of the activity-based fusion, which constitutes one the major contributions of this research. The activity-based fusion combines the data that have been obtained from both sensory and non-sensory sources of information and that have been modified through other levels of fusion and then combines them based on the activities, not based on the objects. The output of this level of fusion is therefore an estimate of the progress of any given activity rather than inferences about the presence of individual objects.

- Level 3 processing, or impact assessment: In general, by providing a number of hypotheses about future events and assessing their impact, this level of data fusion projects the impact of the current situation into the future. In this research, the scheduleupdating feature of the model can be classified in this category of data fusion. The original schedule is used as input for the model, and based on the results of other fusion levels, the estimated progress calculated by the model is used to automatically update the schedule. In this study, the updated schedule is then treated as one of the output results by the fusion model.

- Level 4 processing, or process assessment: As the highest level of data fusion, this level includes processes that modify, control, or manage other processes. The automated generation of progress estimates can be used as input for a number of managerial and administrative processes, and any application that takes advantage of this input would 
thus be classified as belonging to this level of data fusion. Another type of application that can be included in this category is a "what-if" scenario analysis. The model developed in the current research is capable of analyzing "what-if" scenarios with respect to schedule modification, material shipments, etc., all of which are categorized as level 4 data fusion according to the JDL model.

Data fusion processes classified as levels 0 and 1 of the JDL data fusion model have dominated the majority of the research activity related to multisensor data fusion for construction industry applications. However, in many of these applications, such as automated progress estimation for construction projects, an object-based data fusion process is insufficient because many project activities, such as concrete curing, piping installation, welding, inspection, and interior finishing, are not directly associated with a measurable physical entity at the site and therefore cannot be identified using traditional sensing techniques. This difficulty makes object-based models inefficient for estimating the progress of most construction projects.

Although five levels of data fusion are defined in the JDL model, it should be noted that data fusion processes do not need to begin at level 0 or progress in sequence through each level of data fusion. Instead, the order of data fusion processes depends on the type of data collected and the nature of the specific conclusions that must be derived by the model. Data and process management is therefore an important function of data fusion processes at all levels. For multilevel data fusion, data management becomes especially challenging and critical because the model must incorporate various sources and types of information.

\subsection{Research Structure}

The two lower corner boxes in the model described in Figure 3-3 address the two important functionalities within the data fusion and management architecture: process management and database management. The most significant contributions of the research presented in this thesis are the development of these two components for data fusion and management models within the context of the construction industry, which have been missing in previous implementations of JDL model for data fusion applications such as the one presented by Razavi (2009). 
Chapter 5 of this thesis presents a workflow-driven or process-based approach for data fusion for the particular application of construction progress tracking, hence addressing the process management component of the developed data fusion architecture. Then, Chapter 6 presents a workflow-driven or process-based approach to the data management for the application of construction research data management, which addresses the data management component of the model. However, before the fusion engines and workflows could be developed, an evaluation of the available sources of volumetric information as well as a detailed analysis on the performance of the UWB system for positioning information was required in order to characterize the data sources that are used in the fusion processes. These evaluations are presented in Chapter 4. 


\section{Analysis of Data Collection Technologies}

The first objective of this research was to evaluate existing state-of-the-art technologies for data collection on construction projects, to be used in the data fusion model for the purposes of construction progress tracking. This chapter provides a brief summary of the evaluation of the available volumetric data collection technologies, followed by the results of a detailed laboratory and field experimental program on the performance evaluation of UWB system for positioning information. The two evaluations were deemed necessary as part of the data source evaluation presented in Section 3.2.3 and based on the published literature reported in Chapter 2. The results of the evaluations presented in this chapter were required for the development and calibration of the fusion processes presented in Chapter 5 of this thesis.

\subsection{Volumetric Data Collection Technologies}

A review of the literature suggested that both photogrammetry and 3D laser scanning can be used for volumetric data collection using point cloud acquisition on construction projects. However, an objective comparison of the two technologies did not exist in the literature. Since the efficiency of the proposed data fusion model, including its data collection techniques, was one of the objectives of this research, an objective analysis of the two point cloud acquisition techniques was conducted and the results are presented in this section.

The two technologies were compared with respect to their use for an industrial-type building construction project, and the advantages and disadvantages of each technology were evaluated. A number of recommendations for the use of these technologies on construction projects are also provided, with the goal of improving the efficiency and effectiveness of the use of these technologies for construction-related applications. This section provides only a brief summary of this investigation. A detailed comparison of the two technologies has been reported by Ahmed et al. (2011b).

\subsubsection{Background}

Laser scanning technology has been explored as a reliable and accurate method of generating point clouds for the purposes of 3D modelling, as-built model development, and object recognition. Despite the high cost of laser scanning equipment, the rapid scanning ability, 
automated data analysis and precision of this technology have enabled its use in many costeffective applications in a number of fields, such as construction progress tracking.

Photogrammetric techniques have recently been introduced as a potentially more flexible and cost-effective approach for creating point clouds, which significantly reduces the cost of point cloud acquisition and therefore enables a broader range of applications in the construction industry.

In this research, point clouds were to be used specifically for the purpose of automated object recognition for progress tracking. This section presents a comparison of laser scanning and photogrammetry as methods of point-cloud generation, including consideration of costs, portability, labour hours per collected information unit, and training expertise. The analysis was conducted in the context of point-cloud acquisition for the purposes of the volumetric progress tracking of piping and ducting activities for an industrial-type building construction project in Waterloo, ON. The laser scanning was performed using a FARO scanner, and the photogrammetry was conducted by means of a hand-held digital camera and off-the-shelf software called PhotoModeler ${ }^{\mathrm{TM}}$. This section presents a comparison of the strengths and weaknesses of the two technologies when applied for the automated volumetric progress tracking of a construction project over the course of several months.

\subsubsection{Experimental Program for Point Cloud Acquisition}

Point cloud acquisition technologies were compared through the investigation of a real-world case study conducted in a new building under construction on the University of Waterloo campus. The Engineering 6 building was monitored during its construction progress; the experimental program was focused on the fifth floor of the building. One floor was considered large enough to provide a comprehensive basis for the comparison of the available technologies, because it could easily be scaled up to include the entire project or even much larger projects. The following criteria were used for the evaluation of the technologies for the purposes of the research presented in this thesis: portability, training expertise, the labour hours required for data collection and data processing, and the cost of equipment. Since the purpose of this investigation was to compare laser scanning technology with photogrammetry, the experiment was formulated to use both technologies for the production of a point-cloud of a common area in the monitored building. 
For the photogrammetric component of the case study, complicated networks of different types and sizes of pipework were investigated using a low-cost consumer-grade camera: a Canon XSi $450 \mathrm{D}$ with its basic zoom lens. This study used the camera's built-in flash and natural indoor daylight as the primary sources of light. Free positions for camera stations were arbitrarily chosen so that the images taken would maintain a common overlapping area. For the reconstruction of the 3D models, the overlapping was necessary in order to satisfy the coplanarity condition at each model point (Ahmed, Haas, \& Haas, 2011a; Ahmed et al., 2011b). A $3 \mathrm{D}$ point cloud was automatically generated and then processed in order to produce a 3D meshing surface. The surface was rendered either by using the images, so that a virtual-realitystyle surface was reconstructed with the original texture (the first technique), or by using color shades (the second technique). Ahmed et al. (2011a and 2011b) provide further details of the output formats.

The FARO Laser Scanner LS $840 \mathrm{HE}$ that was used in this research is considered an advanced surveying and spatial imaging scanner that uses time-of-flight technology to determine the distance of objects from its mirror and also allows the collection of millions of points with a high spatial resolution. Table 4-1 shows the technical specifications of the laser scanner.

Table 4-2 and 4-3 summarize the specifications of the lens and camera used, respectively.

Table 4-1: Technical Specifications of the Laser Scanner

\begin{tabular}{ll}
\hline Range & $0.6 \mathrm{~m}$ to $40 \mathrm{~m}$ \\
Resolution & $0.6 \mathrm{~mm}-17$ Bit Range / 9 Bit \\
& Intensity \\
Measurement Speed & $120000 \mathrm{~Hz}$ \\
System Distance Error & $+/-3 \mathrm{~mm}$ at $20 \mathrm{~m}$ \\
Laser Power & $20 \mathrm{~mW}$ \\
Wavelength & $785 \mathrm{~nm}$ \\
Beam Divergence & $0.025 \mathrm{mrad}$ \\
Beam Diameter at Exit & $3 \mathrm{~mm}$, circular \\
Vertical Field of View & $320^{\circ}$ \\
Horizontal Field of View & $360^{\circ}$ \\
Weight & $14.5 \mathrm{~kg}$ \\
Ambient Temperature & $+5^{\circ} \mathrm{C} /+40^{\circ} \mathrm{C}$ \\
\hline
\end{tabular}


Table 4-2: Technical Specifications of the Lens

\begin{tabular}{ll}
\hline Maximum Format Size & APS-C \\
Focal Length & $\begin{array}{l}18 \mathrm{~mm}-55 \mathrm{~mm}, 35 \mathrm{~mm} \text { equivalent focal } \\
\text { length }(29 \mathrm{~mm}-88 \mathrm{~mm})\end{array}$ \\
$\begin{array}{l}\text { Diagonal Angle of View } \\
74^{\circ}-27^{\circ}\end{array}$ & $\mathrm{F} 3.5-5.6 / \mathrm{F} 22-38$ \\
$\begin{array}{l}\text { Maximum/Minimum } \\
\text { Aperture }\end{array}$ & 11 elements $/ 9$ groups, 1 aspherical element \\
$\begin{array}{l}\text { Nens Construction } \\
\text { Blades } \\
\text { Minimum Focus }\end{array}$ & 6 \\
Maximum Magnification & $0.25 \mathrm{~m}$ \\
Auto Focus Motor Type & $0.34 \times$ at $55 \mathrm{~mm}$ \\
Focus Method & Extending front element \\
Image Stabilization & 4 stops, single mode \\
Weight & $200 \mathrm{~g}$ \\
Dimensions & $68.5 \mathrm{~mm}$ diameter $\times 70 \mathrm{~mm}$ length \\
\hline
\end{tabular}

Table 4-3: Technical Specifications of the Camera

\begin{tabular}{ll}
\hline Sensor & 12.2 million effective pixels, $22.2 \mathrm{~mm} \times 14.8 \mathrm{~mm}$ CMOS \\
sensor \\
Focus Modes & Al focus, one shot, Al Servo \\
Shutter Speed & $30 \mathrm{~s}-1 / 4000 \mathrm{~s}$ \\
Drive Modes & Single, continuous: $3.5 \mathrm{fps}$, Self-timer $10 \mathrm{~s}(2 \mathrm{~s}$ with mirror \\
& lock-up) \\
Dimensions & $129 \mathrm{~mm} \times 98 \mathrm{~mm} \times 62 \mathrm{~mm}(5.1 \mathrm{in} \times 3.9 \mathrm{in} \times 2.4 \mathrm{in})$ \\
Weight (no battery) & $475 \mathrm{~g}(1.0 \mathrm{lb})$ \\
\hline
\end{tabular}

\subsubsection{Labour hours for Data Collection and Data Processing}

The details of the data collection and data processing procedures for each technology can be found in the work by Ahmed et al. (2011b). An overall summary of these activities is provided in this section. The scanning time for the 3D laser scanner was dependent on the resolution required for the acquired point cloud. Table 4-4 summarizes the scanning time, the number of points in the generated point cloud and the eye safety distance during the scanning for each choice of resolution. 
Table 4-5 summarizes the average estimated time spent on each of the individual tasks that comprised the data collection and processing with both the laser scanning technology and photogrammetry, based on the average of 150 observations.

Table 4-4: Specific Laser Scanner Resolution Characterizations

\begin{tabular}{cccc}
\hline Resolution & $\begin{array}{c}\text { Scanning Time } \\
(\mathbf{m i n})\end{array}$ & $\begin{array}{c}\text { Number of Points in the } \\
\text { Generated Point Cloud (Millions) }\end{array}$ & $\begin{array}{c}\text { Eye Safety } \\
\text { Distance }(\mathbf{m})\end{array}$ \\
\hline $1 / 10$ & 1.11 & 7 & 0.3 \\
$1 / 8$ & 1.74 & 11 & 0.7 \\
$1 / 5$ & 4.44 & 28 & 1.0 \\
$1 / 4$ & 6.94 & 44 & 1.3 \\
$1 / 2$ & 27.78 & 175 & 2.5 \\
1 & 111.11 & 700 & 4.9 \\
\hline
\end{tabular}

Table 4-5: Labour Hours for Data Collection and Processing

\begin{tabular}{lclc}
\hline \multicolumn{1}{c}{ Laser Scanning } & \multicolumn{1}{c}{ Photogrammetry } \\
\hline \multicolumn{1}{c}{ Activity } & Time & \multicolumn{1}{c}{ Activity } & Time \\
Data acquisition training & $2 \mathrm{~h}$ & Data acquisition training & $1 \mathrm{~h}$ \\
Software training & 1 & Software training & 4 weeks \\
& week & & $1 \mathrm{~h} / \mathrm{month}$ \\
Calibration & $\mathrm{N} / \mathrm{A}$ & Camera calibration & $10 \mathrm{~min}$ \\
Establishing the layout & 10 & Establishing the layout plan & \\
plan to scan & min & to photograph \\
Setting up the station & 10 & No station & \\
& min & & $3 \mathrm{~min}$ \\
Putting up the targets & $3 \mathrm{~min}$ & Putting up the targets & $10 \mathrm{~s}$ \\
Point-cloud acquisition & 10 & Picture acquisition (each) & \\
& min & & $5 \mathrm{~s}$ \\
Moving the station to the & $5 \mathrm{~min}$ & Moving between two shots & $10 \mathrm{~min}$ \\
next location & & & \\
Merging 2 scans together & $5 \mathrm{~min}$ & Merging 2 pictures together \\
(data processing) & & (data processing) & \\
\hline
\end{tabular}


The field experiments demonstrated that at least 12 laser scans were required in order to model an entire building floor, whereas 150 to 200 pictures were required when photogrammetry was used.

Figure 4-1 shows the photogrammetry results for the fusion of several interior point clouds (upper left); the generation of CAD pipes and fusion to point clouds (upper right); the output with the first technique: point cloud (lower left); and the output with the second technique: CAD pipe objects (lower right). Figure 4-2 shows the laser scanning results: point cloud (left); fusion of several interior point clouds (right). The point clouds obtained from photogrammetric techniques, unlike those obtained from 3D laser scans, were noisy and not homogeneously distributed in the spatial co-ordinates, which would make modeling and automated progress analysis more difficult using the existing object recognition algorithms as a minimum number of detected points on each object is required in order for the algorithms to detect an object in the point clouds.
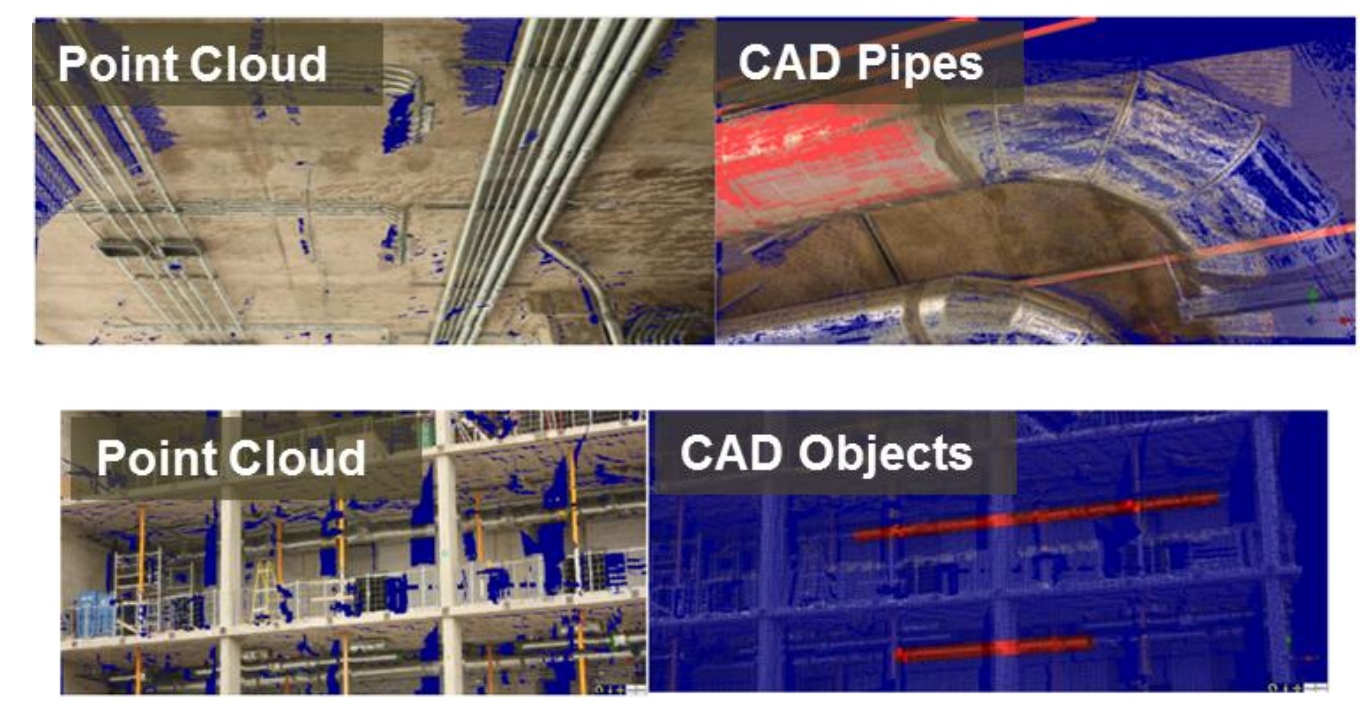

Figure 4-1: Sample Photogrammetry Results 

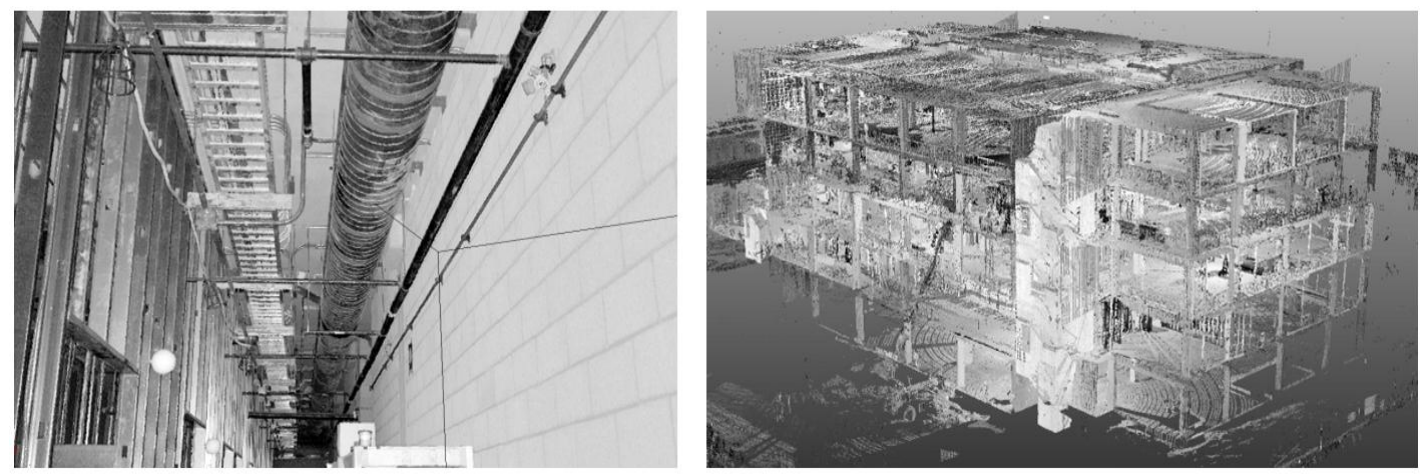

Figure 4-2: Sample Laser Scanning Results

\subsubsection{Equipment Costs}

At the time the experiments were conducted, the price of the camera and lens used for the tests was $\$ 600 \mathrm{CAD}$, and the price of the software was approximately $\$ 3000$ for each seat. The laser scanner itself without any additional equipment was purchased for \$83,000 CAD in 2008. However, a newer, faster, lighter, and more accurate version of the FARO scanner sells for approximately $\$ 40,000$ CAD in 2011 . Advances in scanning technology and the extremely high industry demand for these scanners have been primarily responsible for the decrease in price. Therefore, from a cost perspective, the technologies were not comparable. However, for the research purposes, the technologies were evaluated based on additional criteria other than cost, and overall recommendations were made.

\subsubsection{Portability}

The poor portability of the laser scanning equipment was a significant inconvenience. The technical sheet indicated that the laser scanner weighs $14.5 \mathrm{~kg}$, which does not include the additional equipment required, such as a laptop, targets, an extension cord, and safety gear. If all of this equipment is not stored directly on site, transporting it to the site is very timeconsuming, and in addition, at every change of laser scanner location, the whole station must be relocated. For the tests conducted, Table 4-6 shows the time breakdown with respect to the portability of the laser scanner. These estimates were derived from the average of 150 observations. 
Table 4-6: Time Allocation for Laser Scanner Equipment Portability

\begin{tabular}{ccc}
\hline Step number & Activity & Time spent \\
\hline $\mathbf{1}$ & Establishing the scanning layout plan & $10 \mathrm{~min}$ \\
$\mathbf{2}$ & Setting up the station & $10 \mathrm{~min}$ \\
$\mathbf{3}$ & Putting up the targets & $3 \mathrm{~min}$ \\
$\mathbf{4}$ & Acquiring the point-cloud & $10 \mathrm{~min}$ \\
$\mathbf{5}$ & Moving the station to the next location & $5 \mathrm{~min}$ \\
\hline
\end{tabular}

Given the compactness of the new FARO scanners, which do not even require a laptop, these logistics data and limitations are already obsolete, but are still necessary to report for this research since they were the conditions under which the data were collected.

Because of the obvious portability of digital cameras, the photogrammetric data acquisition consisted almost entirely of the repetition of one step: shooting overlapping images. Shooting a single image usually took only a few seconds. No tripod or additional equipment other than the camera was used during the data collection.

\subsubsection{Technology Constraints}

A number of constraints were associated with the use of these technologies on the construction site. The first set of constraints was related to the weather conditions during which the laser scanner could be used. The ambient temperature had to be between $5{ }^{\circ} \mathrm{C}$ and $40{ }^{\circ} \mathrm{C}$, and the humidity had to be non-condensing. Establishing a safety perimeter around the laser scanner on the construction site also proved to be challenging because of site activities during the scans. The last practical constraint associated with the scanner was the requirement for a power source, which can be problematic during the early stages of construction. This problem has been addressed in new model of the scanner, which uses rechargeable batteries so that a power source is not required. One of the main advantages of the 3D laser scanner was that scene lighting was not a constraint for point cloud acquisition. Also, the new model of the scanner can operate in colder temperatures.

With the photogrammetry technology, the accuracy of the generated point cloud varied with the camera positions and the variations in the relative angles between the camera and the surfaces. 
The quality of the lens also limited the quality of the point clouds. Most importantly, the presence of reflective materials, such as duct work, affected the imaging process due to three main factors: (1) its proximity to the camera itself caused a random dispersion of light; (2) its size compared to that of the other pipe work exaggerated the depth of field compared to the distance of the object from the camera; and (3) the control points were minimal or non-existent and could not be distinguished with the applied configuration, because the ducts acted as irregular mirror surfaces with no control points or appropriate features suitable for the stereo-matching and orientation process. It is recommended that, in similar cases, the following suggestions be implemented: (1) shoot the images from a longer distance; (2) use a wider-angle lens; (3) use a higher-resolution camera to compensate for the effects caused by greater distances, and (4) use a polarizing filter to minimize the mirror effect created by reflective surfaces. Also, emerging software such as 123D CATCH ${ }^{\mathrm{TM}}$ by Autodesk seems to do better with featureless or reflective surfaces, but its results are so distorted as to be useless for the purposes described in this thesis. The use of classical photogrammetric techniques enabled the production of as-built images of the ducts directly from the images independently of the point-cloud generation; however, this is not a feasible approach for progress data acquisition.

\subsubsection{Conclusions and Recommendations}

In conclusion, the laser scanning technology retains a few key advantages over photogrammetry that make it preferable in many situations for the purposes of collecting volumetric information: a shorter training time, a simple and well defined internal coordinate system, a homogeneous spatial distribution of range points, a higher resolution of up to 700 million points, consistent performance regardless of site illumination, and a shorter processing time for the acquisition of a point cloud. However, the laser scanning technology also involves some disadvantages that could potentially shift the balance in favour of photogrammetry: its high purchase cost, and the constraints related to environmental and weather conditions. The cost has been significantly reduced in the newer version of the scanner, and the constraints related to the environmental conditions required for safe performance, such as temperature, have also been improved.

An approach based on the use of photogrammetric point cloud acquisition may include simple data collection with the following advantages: lower cost, convenient portability, absence of eye 
safety or health issues, and robust performance at all temperatures. For the purposes of the research presented in this thesis, the disadvantages of photogrammetry can be grouped under lack of consistency and dependency of the performance of the system on many rather uncontrollable factors. These factors include the experience of the person taking the photographs, the angle of the camera with respect to a variety of objects, the amount of overlap between different images, the experience of the person combining the images, the distance between the camera and the objects, and most importantly, the texture of the surfaces as well as the illumination of the areas where the photographs are taken.

One of the objectives of the current research was to ensure the reliability of the activity-based progress tracking fusion model. The experimental results of the investigation summarized in this section demonstrated that human error can cause a significant reduction in the accuracy levels of photogrammetric-based point clouds. For the specific piping application in this research, the presence of ducts and larger pipes caused a number of difficulties with the photogrammetry application because of light reflection and mirrored surfaces. Another problem with photogrammetry is the lighting. In construction projects, proper illumination for photogrammetry may not be available in all areas and especially not in situations where pipe congestion is heavy. A laser scanner, however, can operate in completely dark environments and is very effective in highly congested areas.

In conclusion, and in consideration of existing state-of-the-art technologies for capturing volumetric information on construction sites as well as recent significant reductions in the price of 3D laser scanners, 3D laser scanners are recommended for point cloud acquisition for the automated construction progress tracking of construction projects. Many other applications related to construction sites, such as as-built modeling and quality control, which are beyond the scope of the current research, would also require the quality of point clouds offered by 3D laser scanners, thus justifying the higher cost of the scanners. It is expected that future improvements in the field of photogrammetry may require this conclusion to be revisited in the future.

\subsection{Positioning Technology: Ultra Wide Band (UWB)}

To further address the first objective of this research with regard to evaluating existing state-ofthe-art technologies for data collection during construction projects with a view to their use in the 
data fusion model for construction progress tracking, this section presents an in-depth evaluation of ultra-wideband (UWB) system for capturing positioning information on construction projects. An extensive review of the literature indicated that UWB positioning can provide a system that is equivalent to the integration of RFID and GPS systems but that, unlike GPS, it can operate both indoors and outdoors. Since the application of the current research was in indoor construction projects, an UWB system was chosen for providing positioning information for the data fusion processes developed in this thesis.

The practical application of UWB technology for construction progress and material tracking involves highly congested and dynamic construction environments. As well, complete occlusion of the UWB tags from the receiver locations occurs due to the presence of construction materials. The literature review of the current research revealed only very limited published work related to the performance of UWB positioning systems in highly congested and active construction environments, and no study has examined the potential deterioration in the performance of a UWB system as construction progresses and the site becomes more congested.

This aspect of the current research was developed in order to investigate the deterioration in the performance of UWB technology in the presence of a variety of occlusions and changing environmental conditions. The specific objectives were to analyze the deterioration in the performance of the UWB system in situations involving complete occlusion resulting from the use of wood and steel as blocking materials and to analyze the UWB system performance over the duration of a progressively more heavily congested piping construction project. To address each of these research objectives, the experimental program was designed in two phases to include a laboratory study and a field experimental study at a construction project. The findings of this investigation demonstrated the effects of occlusions, receiver layout, and changes in the site environment on the magnitude and variability of the measurement error.

\subsubsection{UWB System Specifications}

The UWB system applied in this research consisted of active UWB tags and receivers from the Ubisense ${ }^{\circledR}$ Company. Four types of techniques are generally applied for the purposes of position location: time of arrival (TOA), time difference of arrival (TDOA), angle of arrival (AOA), 
and received signal strength (RSS) (He, Ghavami, \& Aghvami, 2007). The system used in this study takes advantage of both TDOA and AOA techniques, which increased the accuracy of the location estimation.

The particular UWB positioning system used in this research consisted of a network of tags and receivers communicating over $6 \mathrm{GHz}-8 \mathrm{GHz}$ signals. Each tag transmitted an UWB pulse, which enabled the receivers to compute the time difference of arrival and angle of arrival. To locate a tag, the software provided by Ubisense ${ }^{\circledR}$ measured the path from the transmitter to the receiver. Any two pieces of information (e.g., two AOAs or one AOA and one TDOA) enabled the system to compute the position of a tag. Direct path signals determined the true location; however, the reflections of the signal produced a portion of the error within the system. With UWB signals, the reflections could be distinguished from the direct path, and consequently, the system was more accurate. A master receiver that computed the final location of the tag and reported its coordinates to the server was introduced into the system.

\subsubsection{Phase I: Impact of Construction Materials on UWB System Performance}

In the initial stage of this investigation, a preliminary study was conducted to determine the performance of a UWB system with respect to real-time location estimation in a controlled environment in the presence of construction-related obstructing materials. This stage provided a statistical evaluation of the accuracy of the UWB positioning system for a variety of occlusion scenarios.

\subsubsection{Experimental Setup}

The initial phase of the experimental program was conducted in the Infrastructure Systems Sensing Lab at the University of Waterloo. Eight UWB receivers were installed around the perimeter of the lab in a rectangular arrangement and were then connected by CAT-5e shielded wires to one another in a daisy chain and to a master receiver in parallel. The data acquisition system consisted of a laptop connected to a power over Ethernet (POE) switch that was fed by the master receiver. Two types of tags had been provided in the University of Waterloo's UWB system package: compact and slim tags. A compact tag usually attaches above the object to be tracked. The tag's signal update rate can be adjusted depending on its movement speed. The faster the tag moves, the higher the update rate required. A slim tag usually attaches to the side 
of the object and is more applicable when a complex functionality such as a buzzer is needed. The slim tag has programmable buttons and LEDs for different applications. An event can be generated on the server by pressing the button.

Figure 1 depicts a slim tag, a compact tag, and a receiver. The tags were identified by a number on their bar code. The receiver shown in Figure 1(c) represented the master sensor of the network in this investigation, with all of the seven other sensors considered to be slaves. Only compact tags were used in this investigation.
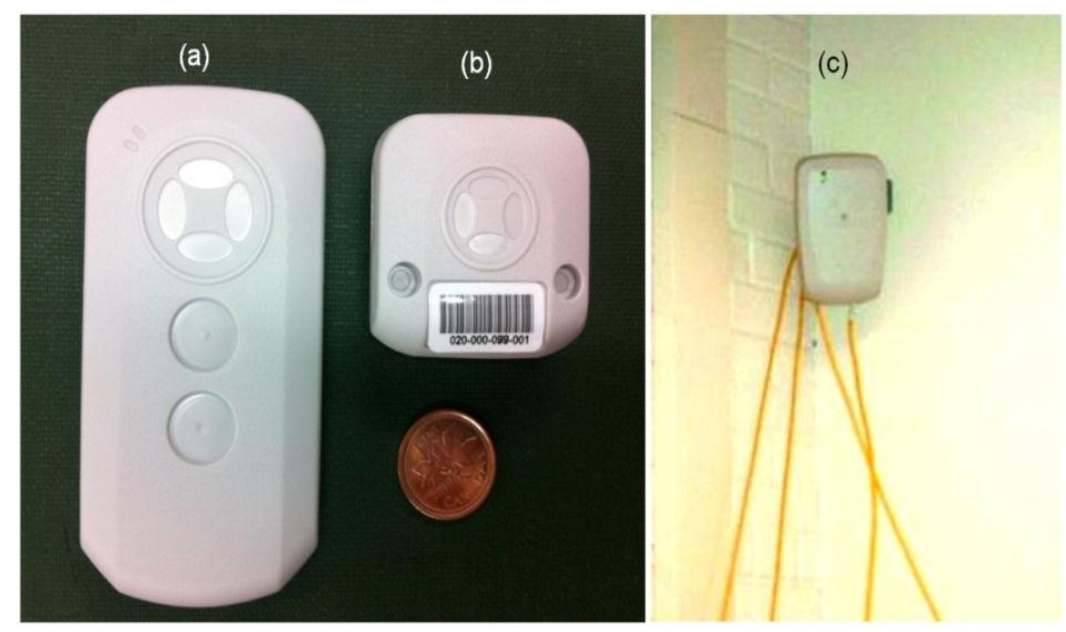

Figure 4-3: (a) Slim Tag, (b) Compact Tag, (c) Receiver

The layout of the sensors in the laboratory is shown in Figure 4-4. This illustration is a screenshot from the licensed software used to monitor the tag. The screenshot depicts the outline of the lab and the receivers that roughly mark the walls. 


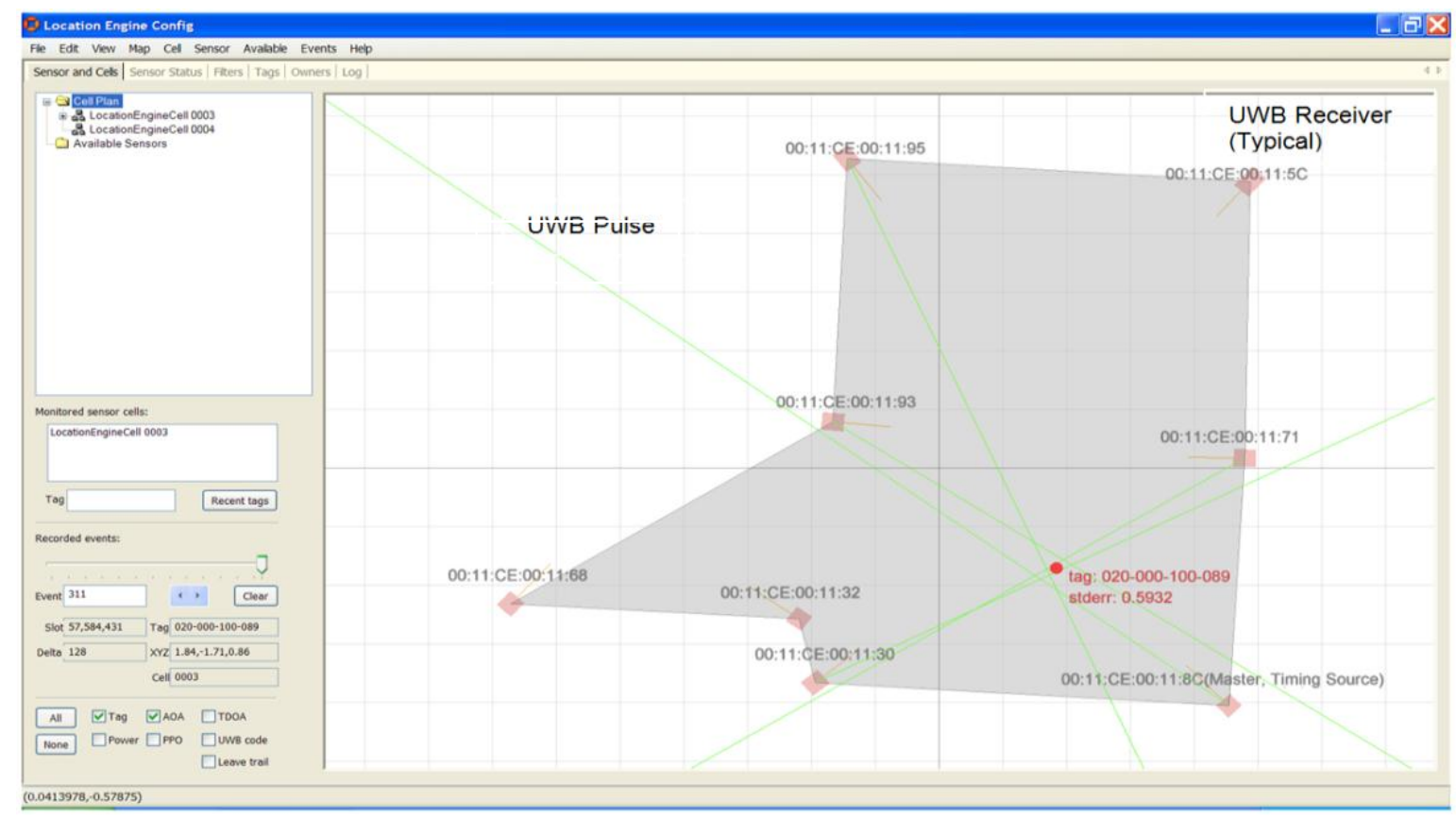

Figure 4-4: Layout of the UWB System in the Lab

The receivers are indicated Figure 4-4 by the red rectangles at the vertices of the shaded region. These receivers were directed inward and downward, thus forming a region within which tags can be sensed. The tag is represented by the red dot from which the green lines radiate. The green lines represent the impulses radiating from the tag to be received by the receivers. The system uses these impulses to calculate the position of the tag in real time. The data acquisition software allowed both real-time and continuous recording of the position of the tag in the area in the form of $(x, y, z)$ coordinates that correspond to the location of the tag at the time of the reading. Due to the real-time nature of the data recording system and the noise present in the data, a number of readings were required for each point in order to obtain an accurate measure of the position for each tag. For this study, a total of 15 measurements were recorded for each tag location.

The obstruction or occlusion of the UWB tags was modeled using closed boxes constructed of either wood or steel to enclose the UWB tags. For a UWB signal to be received from the tag, it thus had to pass through at least one face of the enclosure. The enclosure factor was investigated according to four levels: no enclosure, wood box, metal box, and RF shield box. 
The RF shield box was also metal and was used previously in other research as storage for active RFID tags because it completely blocked the signals from the tags. This enclosure factor was used to investigate the signal penetration of the UWB system versus that of an active RFID system.

The desired response variable in this study was the accuracy of the system. Therefore, all of the readings taken at a given point were compared to the true location of that point, and the difference between the two measurements in three dimensions was considered to be the response variable, or "error." Considering $X_{t}, Y_{t}$, and $Z_{t}$ to be the true coordinates measured by total station surveying equipment and $X, Y$, and $Z$ to be the average of 15 readings of the tag coordinates recorded by the UWB system, the following formula was applied to calculate the response variable, or error:

$$
\text { Error }=\sqrt{\left(X-\mathrm{X}_{\mathrm{t}}\right)^{2}+\left(Y-\mathrm{Y}_{\mathrm{t}}\right)^{2}+\left(Z-\mathrm{Z}_{\mathrm{t}}\right)^{2}}
$$

Four independent measurements, each consisting of 15 individual recorded measurements, were taken for each configuration of the tag enclosure. The response variable was the "average error" for the 15 observations under $i^{\text {th }}$ tag, $j^{\text {th }}$ enclosure, and at the $k^{\text {th }}$ trial, with $\mathrm{i}, \mathrm{j}$, and $\mathrm{k}$ ranging from 1 to 4 . The experimental design of this phase is shown in Table 4-7.

Table 4-7: Experimental Design

\begin{tabular}{|c|c|c|c|c|}
\hline \multirow{2}{*}{$\begin{array}{c}\text { Tag } \\
\#\end{array}$} & \multicolumn{4}{|c|}{ Readings with Each Cover Type } \\
\cline { 2 - 5 } & No Cover & Metal Box & Wooden Box & RFID Box \\
\hline 1 & $E_{1 n 1}, E_{1 n 2}, E_{1 n 3}, E_{1 n 4}$ & $E_{1 m 1}, E_{1 m 2}, E_{1 m 3}, E_{1 m 4}$ & $E_{1 \mathrm{w} 1}, E_{1 \mathrm{w} 2}, E_{1 \mathrm{w} 3}, E_{1 \mathrm{w} 4}$ & $E_{1 r 1}, E_{1 r 2}, E_{1 r 3}, E_{1 r 4}$ \\
\hline 2 & $E_{2 n 1}, E_{2 n 2}, E_{2 n 3}, E_{2 n 4}$ & $E_{2 m 1}, E_{2 m 2}, E_{2 m 3}, E_{2 m 4}$ & $E_{2 w 1}, E_{2 w 2}, E_{2 w 3}, E_{2 w 4}$ & $E_{2 r 1}, E_{2 r 2}, E_{2 r 3}, E_{2 r 4}$ \\
\hline 3 & $E_{3 n 1}, E_{3 n 2}, E_{3 n 3}, E_{3 n 4}$ & $E_{3 m 1}, E_{3 m 2}, E_{3 m 3}, E_{3 m 4}$ & $E_{3 w 1}, E_{3 w 2}, E_{3 w 3}, E_{3 w 4}$ & $E_{3 r 1}, E_{3 r 2}, E_{3 r 3}, E_{3 r 4}$ \\
\hline 4 & $E_{4 n 1}, E_{4 n 2}, E_{4 n 3}, E_{4 n 4}$ & $E_{4 m 1}, E_{4 m 2}, E_{4 m 3}, E_{4 m 4}$ & $E_{4 w 1}, E_{4 w 2}, E_{4 w 3}, E_{4 w 4}$ & $E_{4 r 1}, E_{4 r 2}, E_{4 r 3}, E_{4 r 4}$ \\
\hline
\end{tabular}

\subsubsection{Summary of the Phase I Results}

To determine the statistical significance of the enclosure factor, the average errors associated with different configurations were subjected to a detailed analysis of variance (ANOVA) based on a full factorial experimental design. Since the focus of this thesis was the field 
implementation of a UWB system and its application in a data fusion model for automated construction progress tracking, only a summary of the initial phase of the experimental program is presented in this section. The complete ANOVA tables and the analysis of the tag-to-tag variability and location factor can be found in Aryan et al. (2011). A sample of the results of this phase of the experimental program is presented in Table 4-8. It should be noted that the results presented here are only for one location and that a total of four locations were considered in this experimental program. The analysis of variation (ANOVA) for the "average error" as the response variable is presented in Table 4-9. As indicated in the ANOVA table for the "average error" response variable, the cover factor is very significant, with a confidence level of over $99 \%$ (Aryan et al., 2011). This result was anticipated because blocking the tag with an RFID inhibitor box or even a metal box was expected to have a significant negative impact on the accuracy of the system.

Table 4-8: Sample of Phase I Experimental Results for Average Error (m)

\begin{tabular}{|c|c|c|c|c|c|c|}
\hline Tag \# & $\begin{array}{c}\text { Observation } \\
\#\end{array}$ & None & $\begin{array}{l}\text { Wooden } \\
\text { Box }\end{array}$ & $\begin{array}{l}\text { Metal } \\
\text { Box }\end{array}$ & $\begin{array}{l}\text { RFID } \\
\text { Box }\end{array}$ & $\begin{array}{l}\text { Row } \\
\text { Total }\end{array}$ \\
\hline \multirow[t]{5}{*}{ Tag 3} & 1 & 0.146 & 0.209 & 0.991 & 0.876 & \multirow{9}{*}{5.948} \\
\hline & 2 & 0.167 & 0.208 & 0.240 & 0.474 & \\
\hline & 3 & 0.154 & 0.177 & 0.226 & 0.874 & \\
\hline & 4 & 0.176 & 0.165 & 0.225 & 0.638 & \\
\hline & sum & 0.644 & 0.759 & 1.683 & 2.862 & \\
\hline \multirow[t]{5}{*}{ Tag 1} & 1 & 0.126 & 0.216 & 0.263 & 0.287 & \\
\hline & 2 & 0.121 & 0.169 & 0.209 & 0.494 & \\
\hline & 3 & 0.127 & 0.174 & 0.287 & 0.470 & \\
\hline & 4 & 0.129 & 0.195 & 0.245 & 0.444 & \\
\hline & sum & 0.503 & 0.754 & 1.003 & 1.694 & \multirow[t]{2}{*}{3.953} \\
\hline \multirow[t]{5}{*}{ Tag 4} & 1 & 0.171 & 0.211 & 0.385 & 1.133 & \\
\hline & 2 & 0.189 & 0.202 & 0.223 & 0.852 & \multirow{9}{*}{6.812} \\
\hline & 3 & 0.177 & 0.220 & 0.747 & 0.707 & \\
\hline & 4 & 0.175 & 0.202 & 0.591 & 0.626 & \\
\hline & sum & 0.712 & 0.835 & 1.946 & 3.318 & \\
\hline \multirow[t]{5}{*}{ Tag 2} & 1 & 0.170 & 0.195 & 0.156 & 0.544 & \\
\hline & 2 & 0.135 & 0.179 & 0.174 & 0.655 & \\
\hline & 3 & 0.160 & 0.204 & 0.170 & 0.703 & \\
\hline & 4 & 0.151 & 0.185 & 0.175 & 0.649 & \\
\hline & sum & 0.616 & 0.764 & 0.676 & 2.551 & \\
\hline $\begin{array}{l}\text { Column } \\
\text { Total }\end{array}$ & & 2.474 & 3.112 & 5.308 & 10.425 & 21.320 \\
\hline
\end{tabular}


Table 4-9: ANOVA Results for "Average Error"

\begin{tabular}{ccccccc}
\hline Source & SS & df & MS & Fobserved & $\mathbf{F}_{\text {Critical }}$ & P value \\
\hline Cover & 2.439 & 3 & 0.813 & 43.169 & 2.798 & $1.129 \mathrm{E}-13$ \\
Tag & 0.312 & 3 & 0.104 & 5.528 & 2.798 & 0.002 \\
Interaction & 0.306 & 9 & 0.034 & 1.807 & 2.082 & 0.158 \\
Treatment & 3.058 & 15 & - & - & - & - \\
Error & 0.904 & 48 & 0.019 & - & - & - \\
\hline Total & $\mathbf{3 . 9 6 2}$ & $\mathbf{6 3}$ & - & - & - & - \\
\hline
\end{tabular}

The following equations were used to obtain the SS values in the ANOVA calculations:

$$
\begin{aligned}
& \mathrm{S}_{\mathrm{C}}=\text { Correction for the mean }=\frac{1}{4 \times 4 \times 4}(\text { Grand Total })^{2} \\
& \mathrm{~S}_{\mathrm{D}}=\text { Total Sum of Squares }=\text { Sum of squared observations }-\mathrm{S}_{\mathrm{C}} \\
& \mathrm{S}_{\text {Cover }}=\text { Cover Sum of Squares }=\sum_{i=1}^{4} \frac{(\text { Cover Totals })^{2}}{16}-S_{C} \\
& \mathrm{~S}_{\mathrm{Tag}}=\text { Tag sum of squares }=\sum_{i=1}^{4} \frac{(\text { Tag Totals })^{2}}{16}-S_{C} \\
& \mathrm{~S}_{\mathrm{T}}=\text { Treatment sum of squares }=\sum_{i=1}^{4} \frac{(\text { Replicate } \text { Totals })^{2}}{4}-S_{C} \\
& \mathrm{~S}_{\mathrm{I}}=\text { Interaction sum of squares }=\mathrm{S}_{\mathrm{T}-\mathrm{S}_{\text {Cover }}-\mathrm{S}_{\mathrm{Tag}}} \\
& \mathrm{S}_{\mathrm{Errror}}=\mathrm{S}_{\mathrm{D}}-\mathrm{S}_{\mathrm{T}}
\end{aligned}
$$

However, the ANOVA analysis also revealed unexpected and rather unfavourable results, with respect to the tag and interaction factors also being significant. This unfavourable result indicated that the accuracy of the system may be affected by the particular tag that was used in the system, which reduced the reliability and repeatability of the results that were obtained from any given tag. Further analysis of the data revealed that high fluctuations and pronounced scatter of the readings were responsible for the large number of errors in the system and that the error due to the scatter of the readings in the case of the RFID shield box and metal box dominated the results, which caused the tag and interaction factors to be significant. Detailed analysis of the data for only the configuration with the wood box alone and no cover revealed that if the errors were not dominated by an external variable, there would in fact be no tag-to-tag variations and that the interaction between the factors was non-existent. Figure 4-5 shows the tag-to-tag variations in the results, revealing that no tag-to-tag variations are evident for the 
cases using either the wood box or no cover. The main reason for the domination of results by the high error values is the low number of readings at each point because only 15 measurements were recorded at each location for a tag-cover combination. Based on the results of this phase of the experimental program, it was determined that at least 100 readings should be recorded for each point of data capture, particularly in areas where the direct line of sight between the tag and the receivers is blocked. For phase II of the UWB performance evaluation as well as for the field implementation of the fusion model, 200-500 readings were recorded at each point in order to avoid data domination by high error fluctuations.

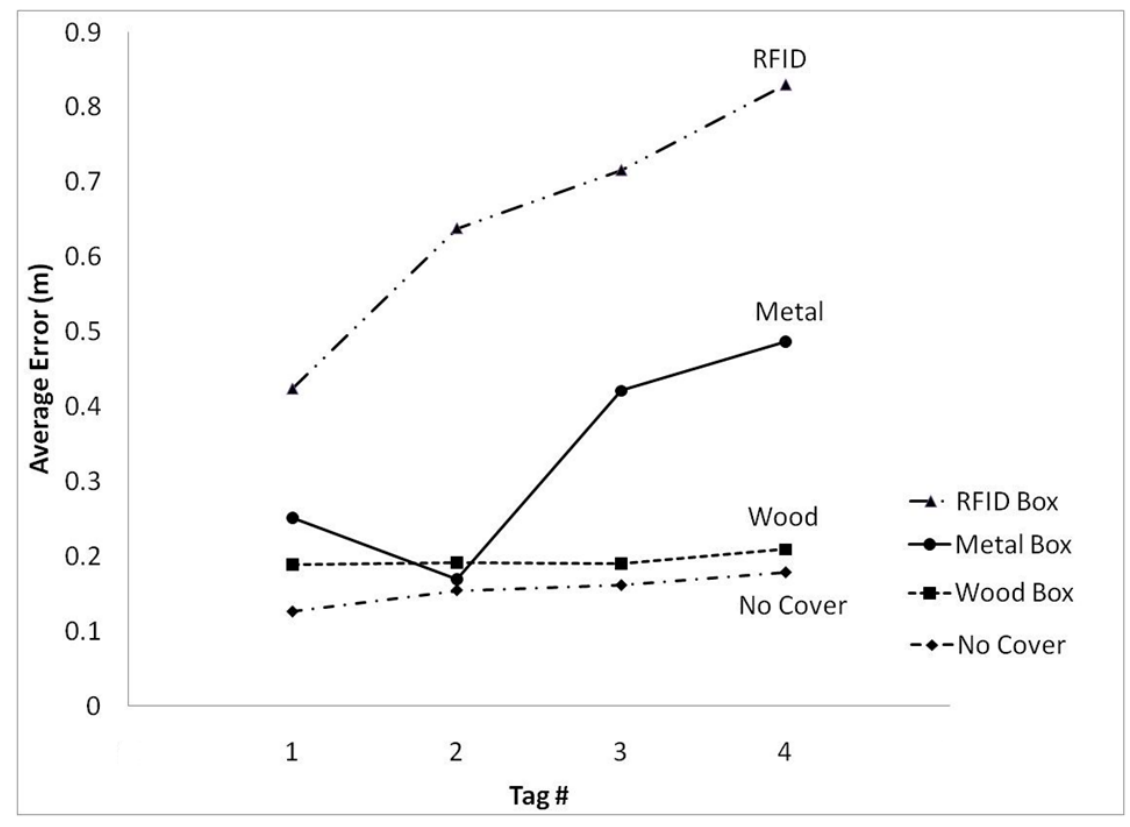

Figure 4-5: Plot of Cell Means for the Average Error

The average errors corresponding to the no cover enclosure and wood box enclosure were relatively low and statistically identical, with average errors of less than $15 \mathrm{~cm}$. The average error associated with the metal box enclosure was much higher: approximately $45 \mathrm{~cm}$. The RFID box readings were highly influenced by scatter and by fluctuations in the readings, and their average was therefore deemed to be inaccurate and unreliable.

Figure 4-6 illustrates the residual plot of the "average error" response variable, in which the residuals have been plotted against the corresponding fitted values. It can be concluded from 
this figure that as the fitted value increases, the residuals increase as well because the value being fitted in this model is, in fact, the "average error." The higher range of the fitted values corresponds to the configurations that result in higher errors: the RFID and metal box covers. Since the standard deviations of the errors that correspond to the RFID and metal box configurations are also higher, the increase in the residual values at the higher fitted values is to be expected.

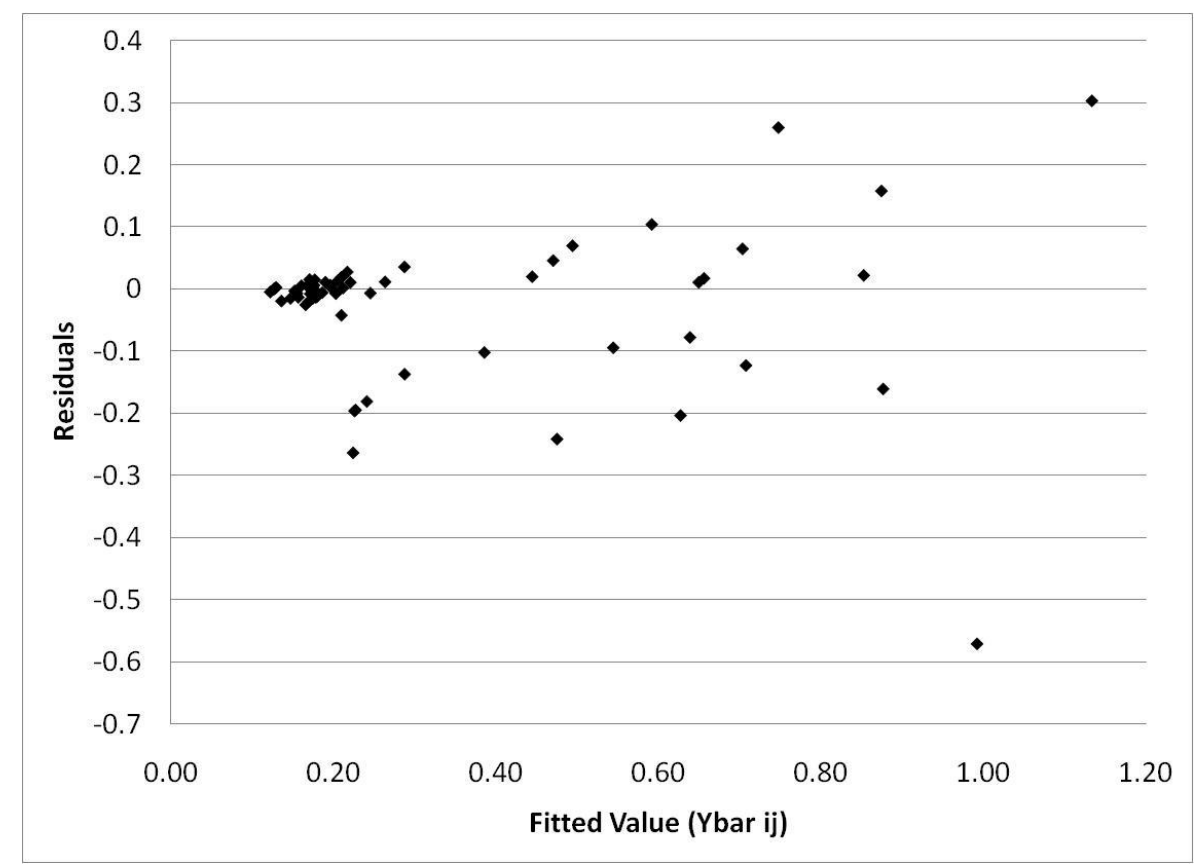

Figure 4-6: Residuals Plotted Against Fitted Values

Figure 4-7 illustrates the UWB measurement scatter plots for the wood enclosure and the metal enclosure. The larger scatter in the metal enclosure data can be explained by the multipath effect caused by the metal surface. Since the line of sight of the tag is blocked in the metal enclosure configuration, the UWB signals reflect from the metal and produce the errors in the measurements. Despite the very large scatter in the metal box data, the average errors are still below $50 \mathrm{~cm}$, which adds to the reliability of the system as long as sufficient measurements are recorded. From the statistical analysis of the results, it was concluded that for situations involving a metal box, a $95 \%$ confidence level of the location estimate of the tag can be obtained within a radius of $1.03 \mathrm{~m}$, and with a no-obstruction configuration, this radius is 
reduced to $0.22 \mathrm{~m}$. The large scatter of the data in the case of metal obstructions was one of the main factors in creating the substantial difference between the two confidence level calculations. Based on these findings, and as explained earlier, in order to reduce the range of the confidence limits, the number of measurements at each point was increased from 15 measurements in Phase I to 200-500 measurements in subsequent phases of this research.

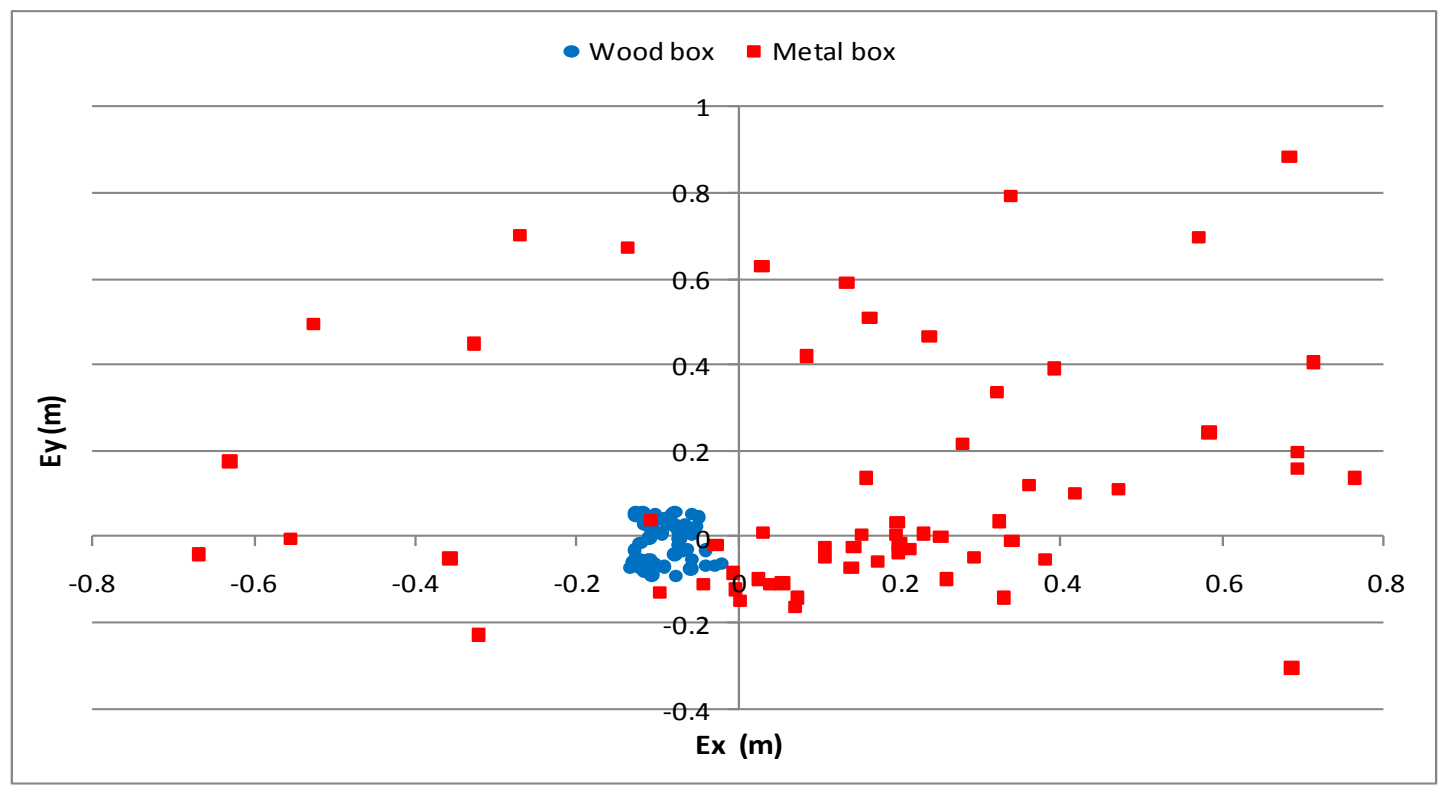

Figure 4-7: UWB Scatter Plot for Wood and Metal Enclosures

\subsubsection{Phase II: UWB System Performance in an Active Indoor Construction Environment}

The objective of the second phase of this investigation was to analyze the performance of the UWB positioning system over a period of several months during a construction project as the work progressed and the site became increasingly congested. For this purpose, the UWB system was installed in a building construction project on the University of Waterloo campus. The building in question housed chemical engineering laboratories and therefore contained electrical, piping, and HVAC services that are similar to those of an industrial or a hospital project. This phase of the UWB investigation and the model implementation presented in Chapter 5 were both completed for the same construction project. 


\subsubsection{Phase II Field Setup and Data Collection}

Four receivers were installed in the fifth floor service core of the building, where the entire piping and ducting network passed through to connect to the HVAC and laboratory exhaust systems in the service penthouse of the building. Due to the linear nature of the service core, the four receivers were mounted on the ceiling along the centre of the service core and spaced approximately evenly over a total distance of $20 \mathrm{~m}$. Each receiver was connected to a POE switch in order to provide power and to transfer data. Different items (pipes, ducts, handrails, and control points on the floor) were tagged and tracked during the construction process. The data was collected during a four-month period (September 2010- December 2010) in order to monitor the progress of the construction as well as to study the changes in the performance of the UWB system as the site became congested. The construction progress of the service core during the data collection period is shown in Figure 4-8, from the time that the receivers were installed (a) until the end of the study (d). It should be noted that in the central walkway in the service core, pipes and ducts are installed through openings in the floor and ceiling on either side of the walkway. The handrails are visible in (c) and (d).

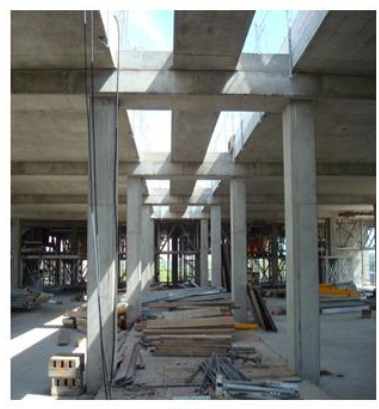

(a)

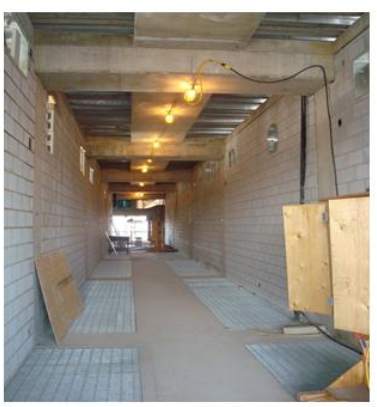

(b)

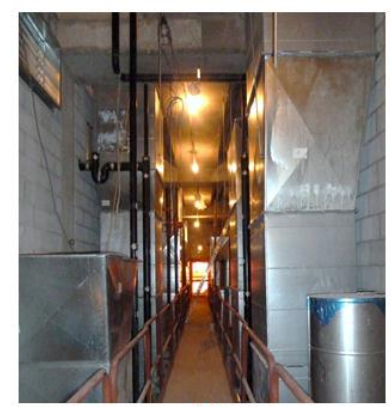

(c)

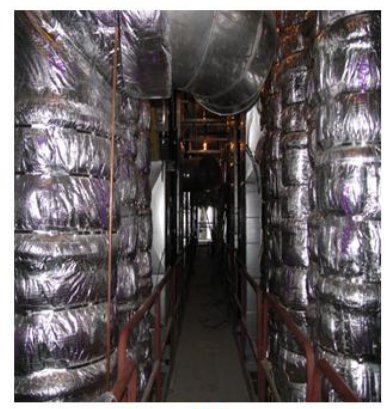

(d)

Figure 4-8: Site Development from the Installation (a) to the End of the Study (d)

More than 30 locations or tag points were monitored regularly over the duration of the experimental program. Eight control points were marked on the floor (termed ground points) and monitored from the start date to the end of the study. Other measurement locations on the pipes, ducts, and handrails were monitored as the items were installed and then for the remainder of the study period. The data were monitored regularly in order to investigate the dependency of the UWB system on the level of construction congestion by assessing whether 
the performance of the UWB system would deteriorate as the site became congested, and if so, to quantify the deterioration.

\subsubsection{Phase II Results and Analysis}

The analysis of the data collected revealed that the UWB system performance of the measurement points located in the middle of the service core (on the floor and on the handrails) was distinctly different from that of the points located on the congested piping areas on both sides of the service core. This difference can be attributed to the fact that the piping measurement points were all located on two sides of the service core, and as construction progressed, some of the measurement locations became partially or completely occluded from the line of sight to one or more receivers. In contrast, all of the floor/ground and handrail points were more centrally located and maintained a line of sight to the receivers.

Figure 4-9 summarizes the readings taken over the duration of the project for all of the ground points. Although the daily readings show scatter and possible outliers, the average threedimensional error remained stable below $0.6 \mathrm{~m}$ over the duration of the test period.

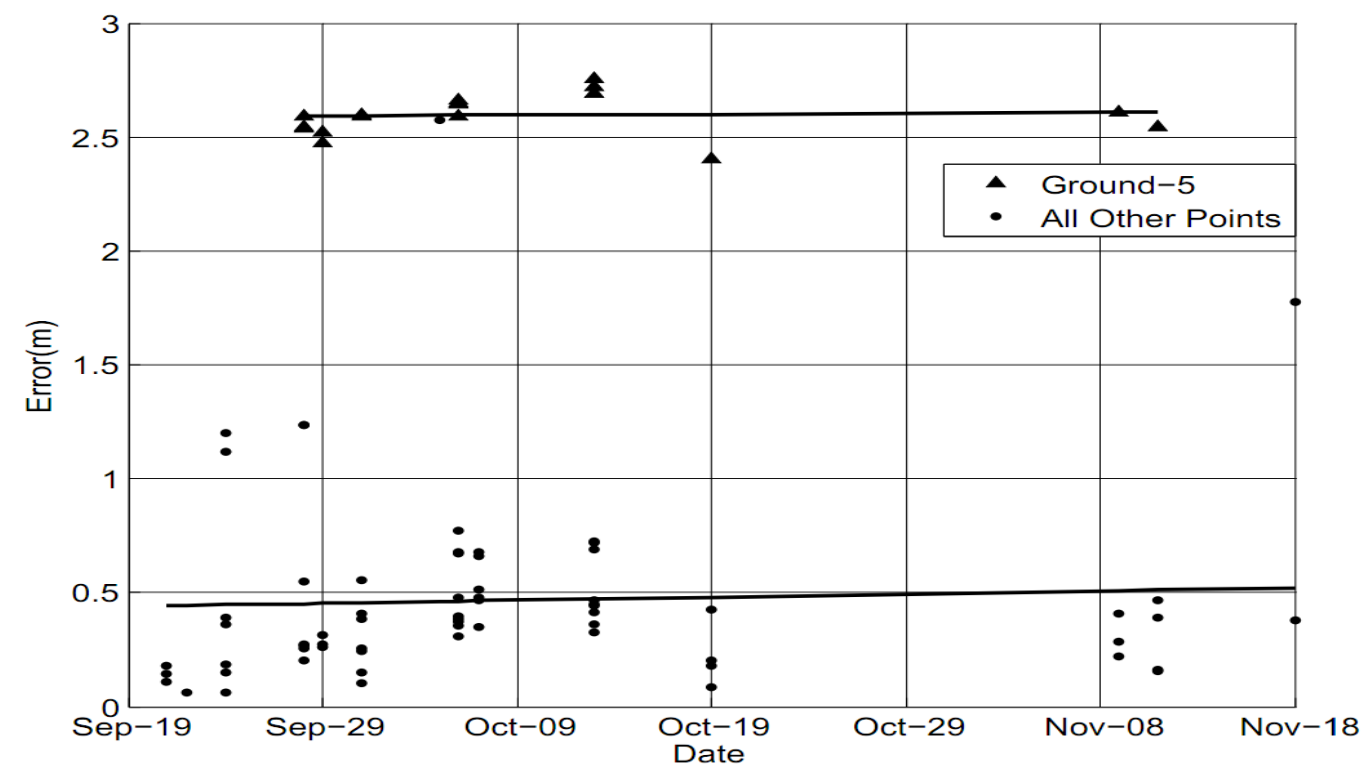

Figure 4-9: Error Trend for Ground Points over Time 
The most interesting observation from this data set is that there is no significant deterioration in the performance of the system for these readings over the duration of the project. This conclusion suggests that as long as direct line of sight is maintained between the tag and the receivers, the increased congestion of the construction environment over time has no significant impact on the performance of a UWB system.

It was also concluded that Ground-5 points had consistently high errors over the duration of the project, potentially due to the heavy presence of metal surfaces in that region as it was positioned right beside a handrail frame. Despite the high error for the Ground-5 points, the error did not change over the duration of the project, which was consistent with the observations from the other ground points.

The performance of the UWB system for the measurement points on the pipes was very different than for those on the floor. Figure 4-10 illustrates the average error of all pipe points over the duration of the project.

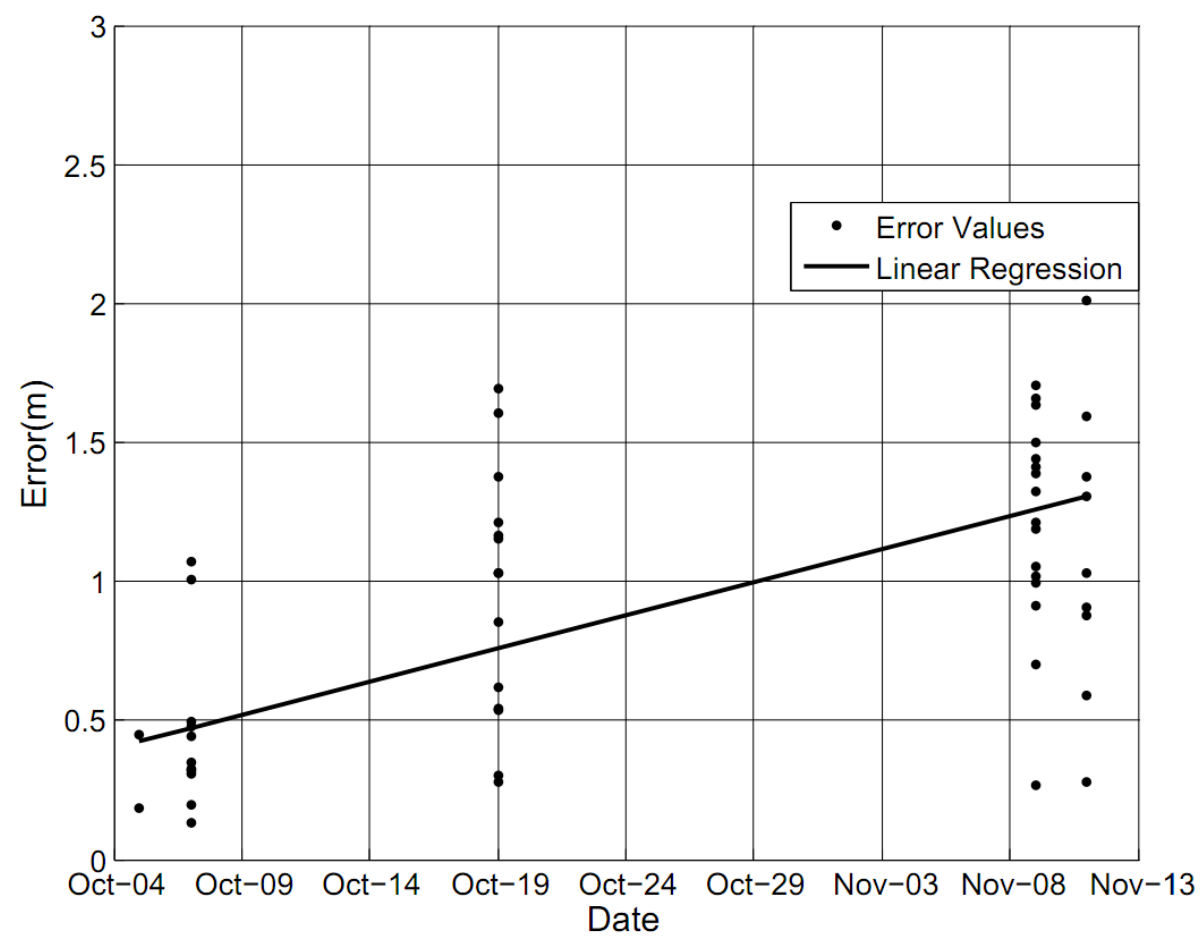

Figure 4-10: Error Trend for Pipe Measurement Points over Time 
It is clear from the regression line that there was a significant increase in the average error as the site activities progressed and pipe congestion increased. The average error for the points on the pipes was initially about $0.5 \mathrm{~m}$, which was consistent with the average error of the floor points presented above. However, as the activities on the construction site progressed and pipes were installed, this error approached $1.2 \mathrm{~m}$. This reduction in system accuracy can be attributed to a reduction in direct line-of-sight signals between the tags and the receivers as construction progressed. It was therefore concluded that, depending on the congestion at the site, the field error could be between $0.4 \mathrm{~m}$ and $1.2 \mathrm{~m}$ for the site conditions evaluated.

Another important observation from the data presented in Figure 4-10 is that the variance of the average errors around the mean also increases for pipe measurement locations. This observation was further analyzed by an examination of the changes in the scatter plots of data for selected locations over time. Figure 4-11 illustrates the scatter plots of the readings associated with three separate points at two different times in the project. These plots clearly illustrate that the magnitude of the average error in the $x$-y plane increased over time. As well, the position of the measured points relative to the true location changed as construction progressed, and the scatter in the data also increased substantially. This increase in the measurement error and scatter of the data as the amount of occlusion and number of obstructions increased was expected based on the findings in the initial phase of the experimental program, in which it was shown that $100 \%$ occlusions caused by metal surfaces increase the error in the data as well as the scatter.

As previously mentioned, the arrangement of the four UWB receivers used in this field study was essentially linear, because the geometry of the service core area and pipe/duct arrangement prevented a rectangular receiver layout. The use of a linear receiver arrangement is possible since UWB technology uses both the time difference of arrival (TDOA) and angle of arrival $(A O A)$ techniques to improve the accuracy of tag location. The four receivers were well spaced along the entire length ( $x$-axis) of the service core. However, the receivers were installed at the same elevation (no variation in z-axis location) and approximately along the centre of the service core (minimal variation in y-axis location). The consequence of this essentially linear receiver arrangement on the magnitude and variability of measurement errors is apparent based on an examination of the error in the three principal directions. 

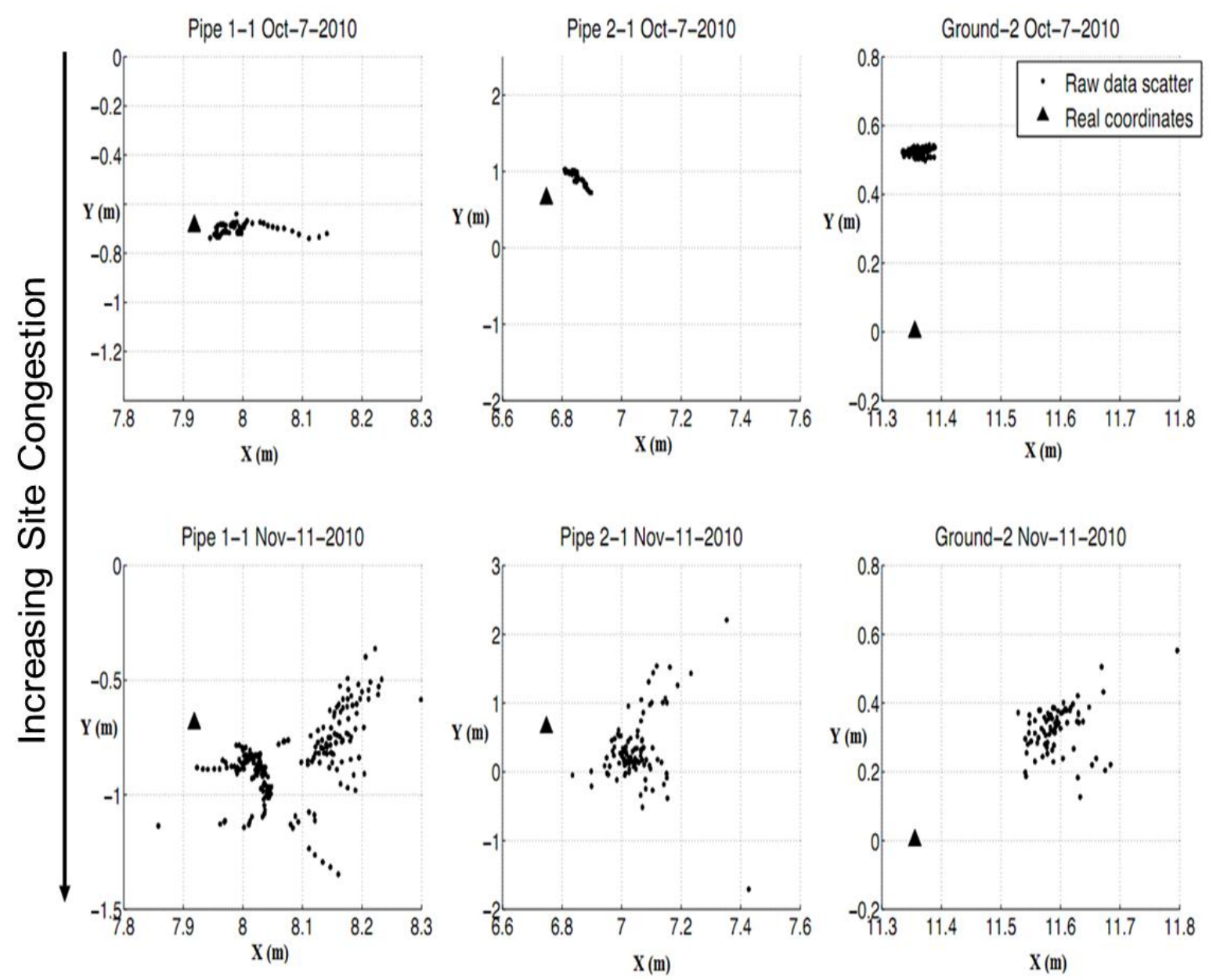

Figure 4-11: Scatter Plots over the Project Duration for Selected Locations

Figure 4-12 shows the 3D scatter plot of a point on Pipe 2 (left) along with the scatter in the $x-y$ plane (middle) and $y-z$ plane (right). The results indicate a clear difference between the errors in the $x$-direction and those in the $y$ - and $z$-directions; the magnitude of the error in the $x$-direction and the measurement scatter are considerably less than those measured in the $y$ - and $z$ directions. Analysis of the data collected from all the points reveals a consistent trend of $E_{z}>E_{y}$ $>E_{x}$. These results illustrate the effect of the receiver layout on the measurement error: the spatial distribution of the receivers was largest in the $x$-direction, resulting in a reduced measurement error compared to that in comparison to the $y$-and $z$-directions, where the spatial distribution of the receivers was limited. 


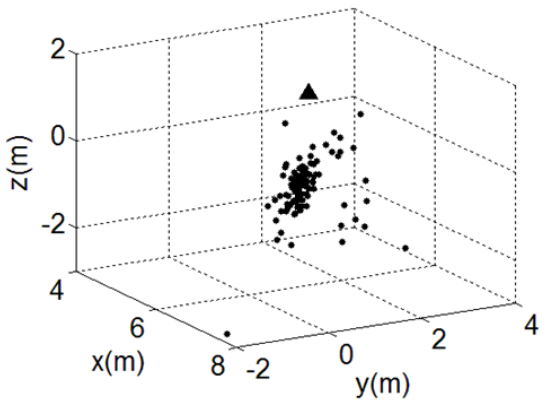

(b)

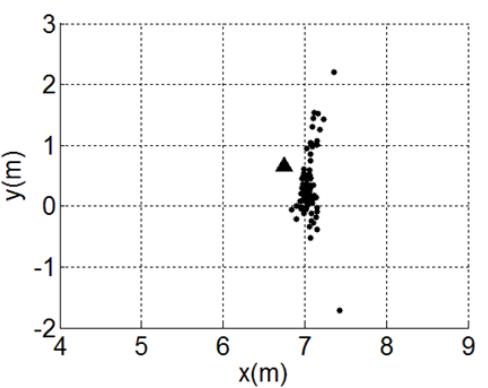

(c)

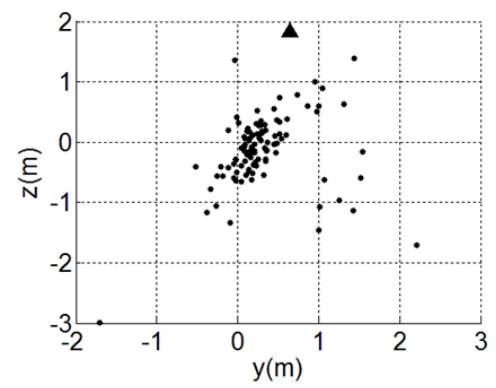

Figure 4-12: Scatter Plots for Pipe 2-1

\subsubsection{Field Implementation Recommendations}

Error Correction: Based on investigations of the performance of UWB systems reported in the literature and on the findings of the initial phase of this investigation, it appeared that locationbased error modeling and correction may be employed to improve the accuracy of UWB position data. However, the conclusions based on the field implementation study in the current research were that in active construction environments where site conditions are changing and becoming more congested, both the magnitude and direction of the error changes over the duration of the construction. Although it may therefore be seem beneficial to perform a detailed error-modeling study at the beginning of the project to allow the correction of site-specific systematic errors, the calibration process would need to be repeated as the site conditions change. In other words, for active construction environments, location-based error modeling and correction may not be reliable, accurate, or even feasible.

Layout of the Receivers: An examination of the error in the three principal directions illustrated the dependency of the amount of the error on the receiver layout. The optimal receiver layout should incorporate spatial variability of the UWB receivers in the three principal directions. Rectangular or polygonal receiver arrangements are therefore preferable to linear or otherwise constricted layouts. Where possible, it is also desirable to incorporate spatial variability in the zdirection (height) of the receiver locations. The configuration of the building service core study area in the current research restricted the receiver installation to a straight line, which reduced 
the accuracy of the system in the $y$ - and z-directions. Where the geometry of the measurement space restricts the spatial distribution of the receivers, it is essential that the measurement error be carefully characterized in the principal directions in order to determine whether the resulting accuracy is appropriate for the intended application.

\subsubsection{Conclusions}

UWB positioning is an emerging technology that is being investigated for a number of potential applications in the construction industry. It has been applied for material and progress tracking in indoor environments as well as for a variety of safety-related applications.

In the initial phase of this research, the performance of UWB technology in the presence of construction-related obstructing materials was analyzed. Analysis of variance for the average error showed that the performance of the system is strongly affected by the presence of occlusions. The results indicate that, with $100 \%$ enclosure of the receivers with a metal surface, the average error is about $50 \mathrm{~cm}$, and the location can be estimated within a $1 \mathrm{~m}$ radius at a $95 \%$ confidence level. The results further show that $100 \%$ enclosure with wood has no negative impact on the performance of the system. For no enclosure and wood enclosure configurations, the average error is below $15 \mathrm{~cm}$, and location can be estimated within a $22 \mathrm{~cm}$ radius with $95 \%$ confidence.

The second phase of the research was designed to analyze the potential deterioration in the performance of the UWB system over the duration of an indoor construction project in order to investigate the applicability of this technology for a range of applications, including material and progress tracking. Despite the non-optimal configuration of the UWB receivers that was imposed by the unique layout of the site, the results indicate that UWB can be used effectively for a wide range of construction-related applications. It was concluded that as long as direct line of sight is maintained, increasing site congestion over time has no impact on the measurement error of the system. It was further concluded that when direct line of sight is non-existent and occlusions reach $100 \%$ because of the presence of many metal pipes and ducts, the accuracy of the system deteriorates substantially. Specifically, the average error increases from $0.4 \mathrm{~m}$ at the start of the construction with no occlusions, to $1.2 \mathrm{~m}$ at the end of the construction with multiple occlusions. The magnitude of error is greater than that obtained from the laboratory 
phases of the current research, due primarily to the non-optimal linear layout of the receivers in the field experiments. Analysis of error in the principal directions indicates that the magnitude and variability of the error are related to the spatial variability of the receiver arrangement. This finding illustrates the importance of receiver layout design and site-specific error characterization. The data also show that not only does the magnitude and variability of the error increase as the site becomes congested, but the direction of the error may also change. Therefore, location-based error correction may not be reliable, or even feasible, for active construction environments unless error modelling is performed on a regular basis and the correction algorithms are updated. 


\section{Data Fusion Model Development and Implementation}

This chapter presents the workflow-driven fusion algorithms that are integrated within the overall fusion model of this research. First, the fusion strategy that was used in the data fusion architecture of this research is presented followed by the setup for field experiments. The innovative indicator-tag approach that was developed in this research for tracking activities on construction projects is presented next. Then, the details of the various fusion engines and algorithms of the model are presented within the developed workflow driven framework. This chapter concludes with a performance evaluation of the developed model with respect to the existing progress tracking methods on construction projects and other data fusion models developed for the application of automated construction progress tracking.

\subsection{Fusion Strategy}

With the dramatic increase in computer power over the last two decades, researchers in the data fusion community have exhibited a tendency to take advantage of the enormous processing power now available. At the raw data level, which corresponds to the lowest level of fusion in all existing models, data fusion processes require significant processing power. By leveraging the increased computer power, researchers in this field have been able to demonstrate the advantages of data fusion compared to traditional, manually intensive procedures, particularly in the field of project progress estimation and material tracking (Bosché, 2010; El-Omari \& Moselhi, 2011; Golparvar-Fard et al., 2009; Razavi \& Haas, 2010). However, humans still have a critical advantage over computers: their ability to make decisions from a global perspective. It was not the intent of this research to develop a fusion model that relies on the immense power of computers to process raw data. Rather, this research has developed a fusion architecture that can provide a more global and practical perspective of progress on construction projects and that is capable of assisting with decision-making processes based on the sources of data and information available at any given time. The workflow driven architecture of the fusion model further formalizes the fusion processes and ensures the integrity, consistency, and reliability of these processes.

Most researchers in the sensor fusion community insist that the lowest possible level of data fusion is always preferable because the level of detail is greatest at those levels of the fusion 
process (Dasarathy, 1997). However, at higher levels, fusion involves lower computational complexity and fewer registration problems between various sources of information, which enables the combination of volumetric data, positioning data, and project control information. Due to the nature of construction sites, data at the sensor level is rather noisy, inaccurate, and incomplete. Also, numerous other modes of construction data information are available that are not classified as sensors, and hence cannot be utilized in low-level data fusion models.

Examples of such sources of information include schedule data, work orders, and progress reports. The data fusion methodology developed in this thesis is therefore based on data fusion that is classified as higher levels according to the JDL model. Figure 5-1 illustrates the fusion levels that were incorporated as part of the data fusion engines presented in this chapter. The process management focuses on the data fusion workflows within the model, while levels 1 to 4 of the JDL fusion model deal with the collected volumetric, positioning, and project control data fusion algorithms.

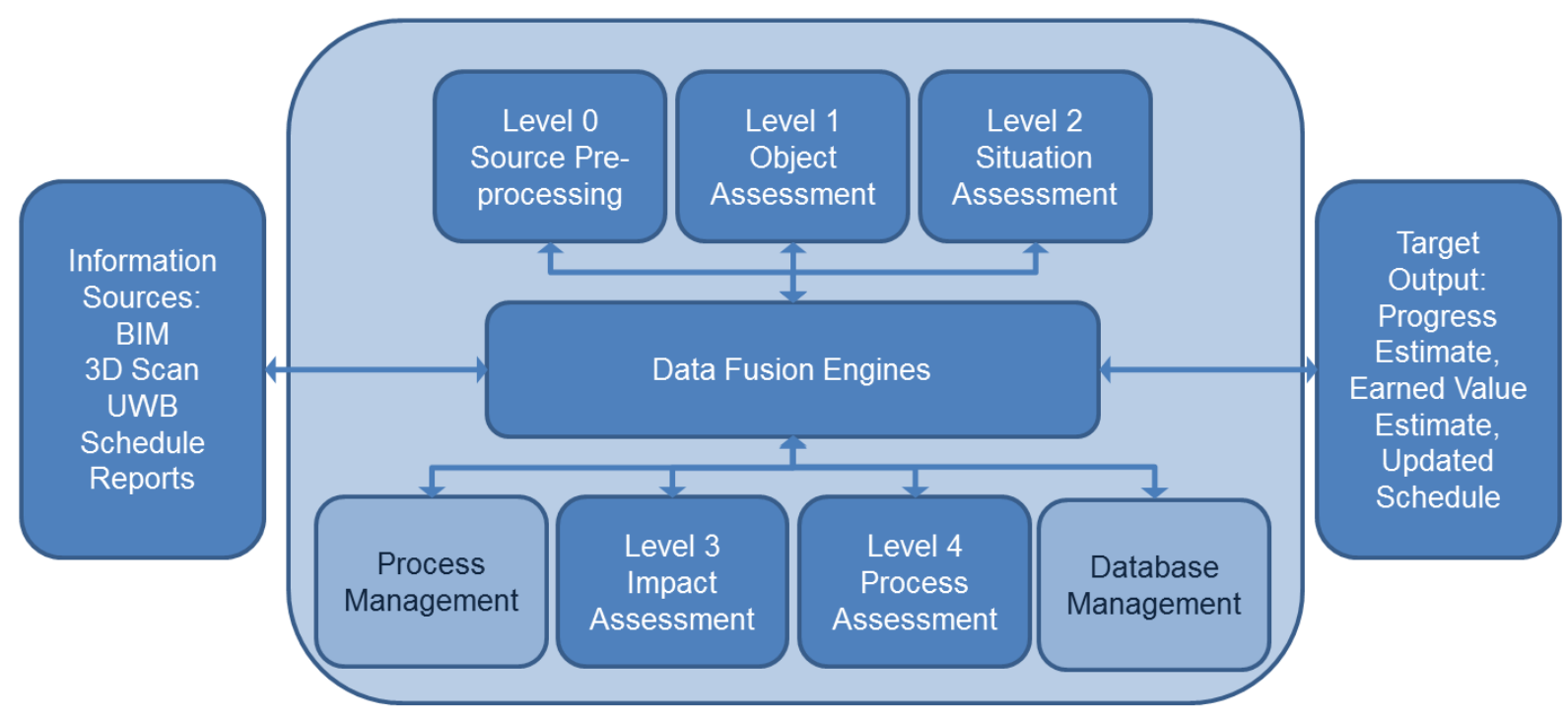

Figure 5-1: Data Fusion Processes within the Developed Model

\subsection{Field Setup and Data Collection}

The model that was developed in this research in order to provide automated progress tracking of construction projects was validated in part with the use of the data collected over a period of several months from a building construction project on the University of Waterloo campus. The 
field experimental program, and therefore the validation of the model, had to be conducted in installments because the model was being developed and calibrated simultaneously with the data collection stage. It is therefore important that the circumstances and extent of data collection be discussed before the presentation of the fusion algorithms and processes developed for this research.

The building in question housed chemical engineering laboratories and therefore contained electrical, piping, and HVAC services similar to those found in an industrial project. The scope of the experimental program was limited to the piping and ductwork activities on one floor of the building. The piping systems were chosen because they include activities directly associated with physical entities, such as delivery and installation, as well as other non-structural activities such as welding and inspection.

The field experiments were completed on the project that was previously introduced in section 4.2.3 of this thesis. Figure 5-2 shows the three-dimensional CAD model of the fifth-floor room layout and the associated piping layout. Each floor of the building contains centrally located chemical engineering laboratories, with offices arranged around the perimeter of the building. A central service core collects and distributes all water, air, gas supplies, the HVAC system, and the laboratory exhaust systems.

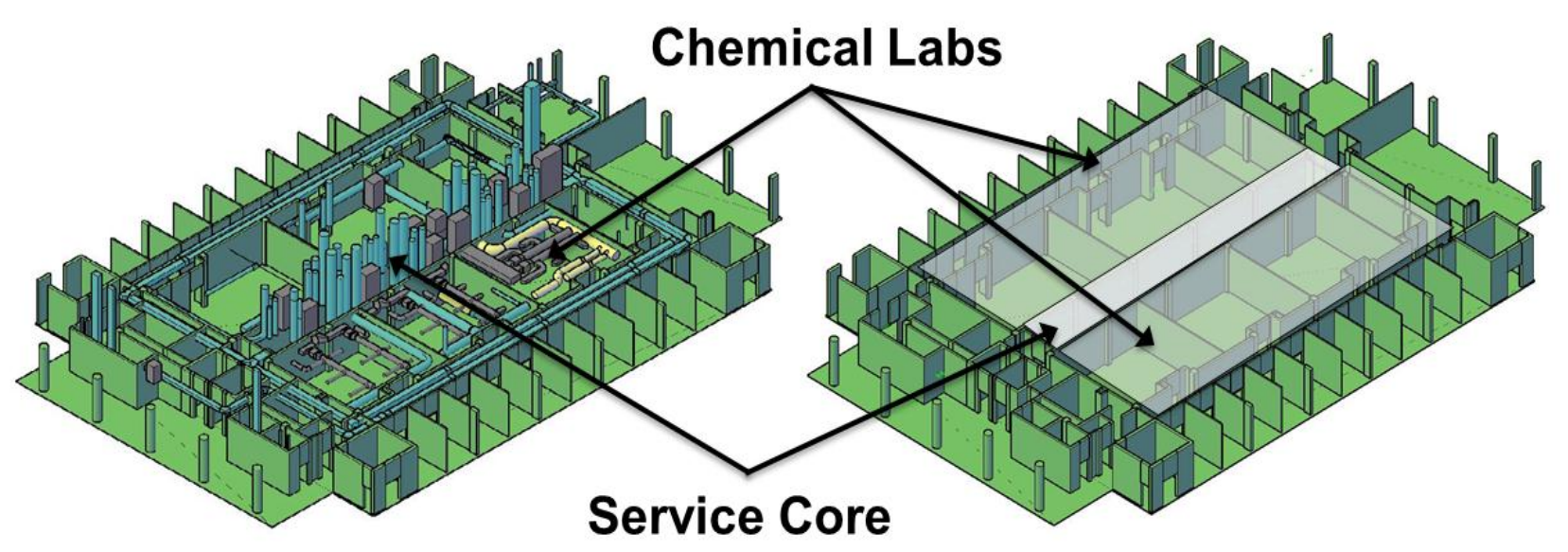

Figure 5-2: 3D CAD Model of Fifth-Floor Piping Layout (left) and Plan (right) 
The service core was selected for the field implementation of the UWB system due to the high volume of piping and ductwork located there, which provided an opportunity to assess the performance of the model in an environment in which congestion would steadily increase over the course of the project. The conditions were also similar to those found in industrial or energysector construction projects, allowing the performance of the fusion model to be assessed for possible future use in those applications.

Four UWB receivers were installed in the fifth-floor service core of the building, where the entire piping and ductwork network passes through to connect to the HVAC and laboratory exhaust systems in the building service penthouse. Full details of the UWB field setup and data collection procedures are included in section 4.2.3 of this thesis. Figure 5-3 shows the progress of the building (left) and the condition of the fifth-floor corridor (right) at the beginning of the data collection and field experiments.
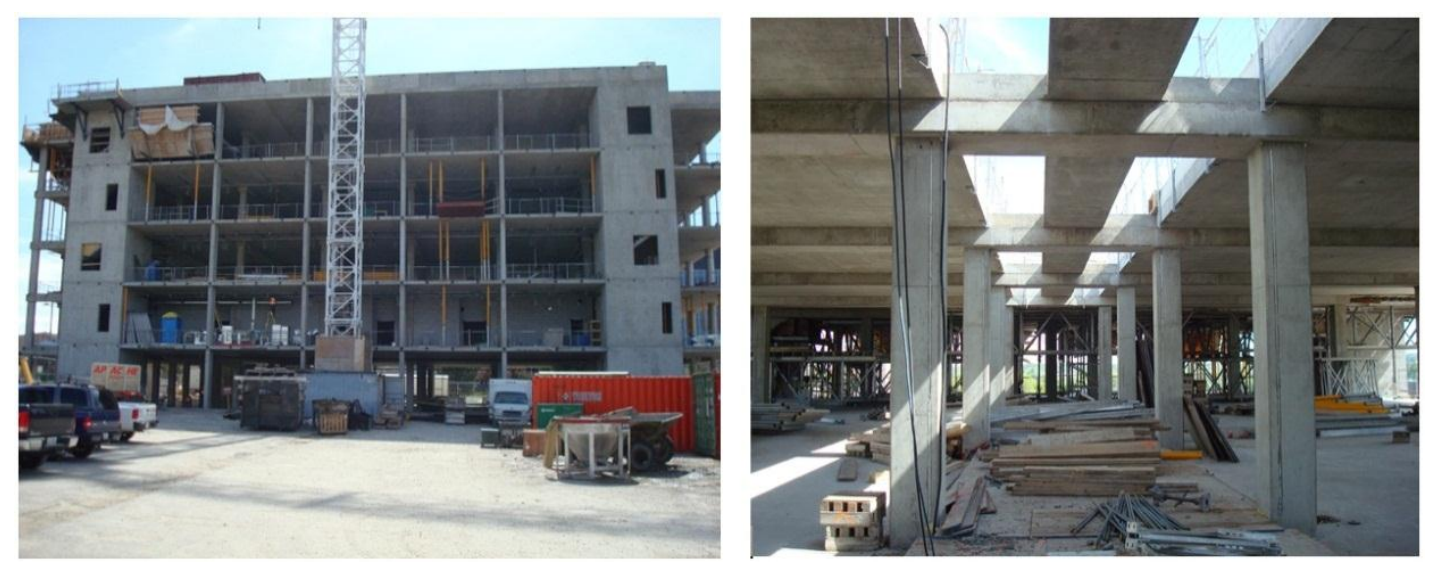

Figure 5-3: Site Conditions at the Beginning of the Data Collection

The 3D laser scanning data were captured during a five-month period from August to December 2010 using the FARO scanner previously described in section 4.1. Figure 5-4 shows the field setup for the FARO laser scanner. A total of 93 laser scans were taken from the fifth floor of the building during this time, in addition to 26 scans from the fourth-floor corridor and 12 scans from the third-floor corridor. Since most of the pipe and duct lines travel vertically between corridors, the scans from the third and fourth floors were taken in order to obtain a better picture of the 
progress of the piping networks. Each scan file included up to 40 million points, which translated into approximately $100 \mathrm{MB}$ of data for each scan.

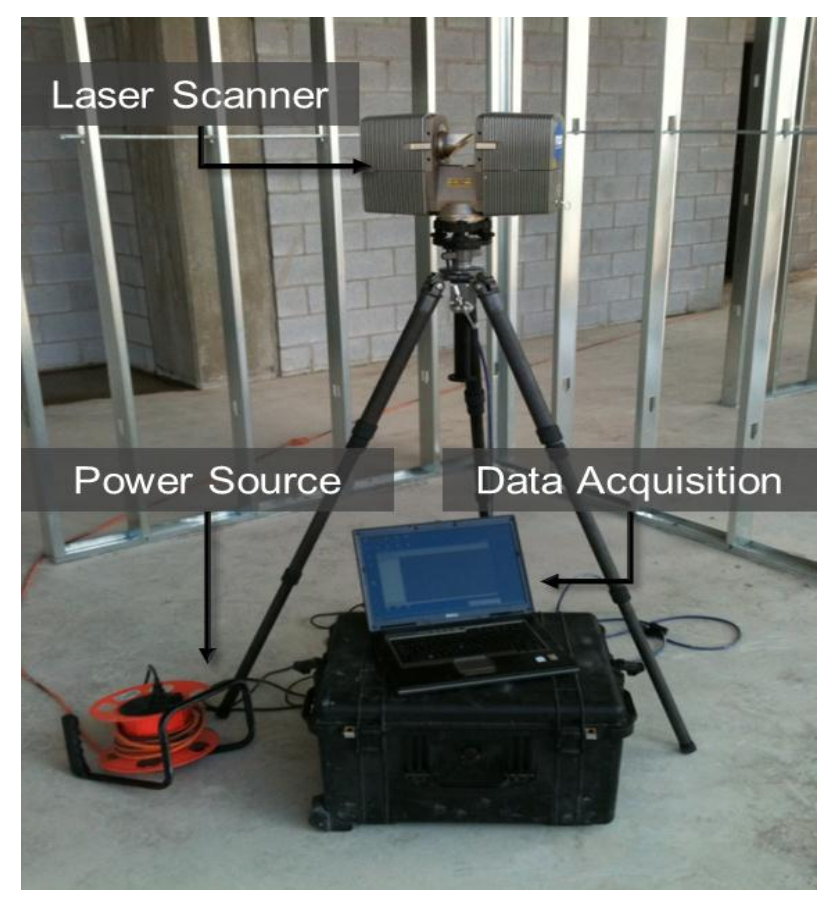

Figure 5-4: Field Setup for the Laser Scanner

The administrative documents that were collected included the construction schedule from the general contractor; all 2D drawings for the structural, mechanical, and electrical trades; and all progress reports in the form of payment applications made to the general contractor. There were no other sources of progress tracking on this project beyond the payment applications submitted by the sub-contractors to the general contractor. From the 2D CAD drawings that were provided by the contractor, a 3D model was built by a co-op student who was working on this research project. To serve as the as-built 3D model for the building, a separate 3D CAD model was also constructed based on the original model but modified using the information from 3D laser scanner point clouds. The following sections provide the algorithms, processes, and strategies that were used to combine these sources of data in order to obtain a progress estimate of the project. 


\subsection{Innovative Indicator-tag Approach for Activity Progress Tracking}

An important data collection method for the data fusion processes developed in this research was the innovative indicator-tag approach that was developed for tracking activities on construction projects. Activity-based tracking is necessary in order to quantify the progress of activities that are not directly associated with the addition or removal of physical entities on a site, such as the welding or inspection of pipe-spools. There were no existing methods to track these activities on construction projects and therefore the developed indicator-tag approach for tracking activities on construction projects is one of the unique contributions of this research.

This technique is described in this research within the context of piping activities, but it is applicable to a wide range of other activities, including interior finishes, concrete curing, etc. In the context of piping activities, the activities of installation, welding, and the inspection of the welds for a pipeline are each linked to a unique UWB tag ID so that the activity-tracking data for each activity is obtained by matching the tag ID that was captured to a list of predetermined tag classifications. For example, welders place a preconfigured tag on a pipe as soon as the welding is completed. The identification of the tag by the UWB monitoring system signals that the welding activity has been performed on a given pipe, at the location of the tag (3D position) that has been recorded by the system. A path is established from the discrete readings for each activity, and the length and location of these paths are used to enable the activity progress estimation in the fusion model, by cross-matching the measured path lengths with the asplanned path lengths from the CAD model. Figure 5-5 shows schematically how the UWB activity-tracking data were captured for the pipeline work on the experimental project. As the pipeline was installed (right picture), UWB marker readings were taken (shown by gray dots) to be used as a means of estimating the progress of the installation activity. Using this strategy, each welder or inspector would need only one or two tags, depending on the number of different welds they have to complete. Therefore, a welder who must weld a natural gas pipeline would carry two tags to differentiate the welds on the $10 \mathrm{~cm}$ and the $15 \mathrm{~cm}$ diameter pipes.

For Activity Progress Tracking, the average location of the coordinate scatter of each file is stored within the hierarchical array, as shown in Figure 5-6. The vertical arrows indicate the hierarchy of data arrangement for sorting and retrieval, and the horizontal arrows indicate the activity-based tracking path created from each file. 

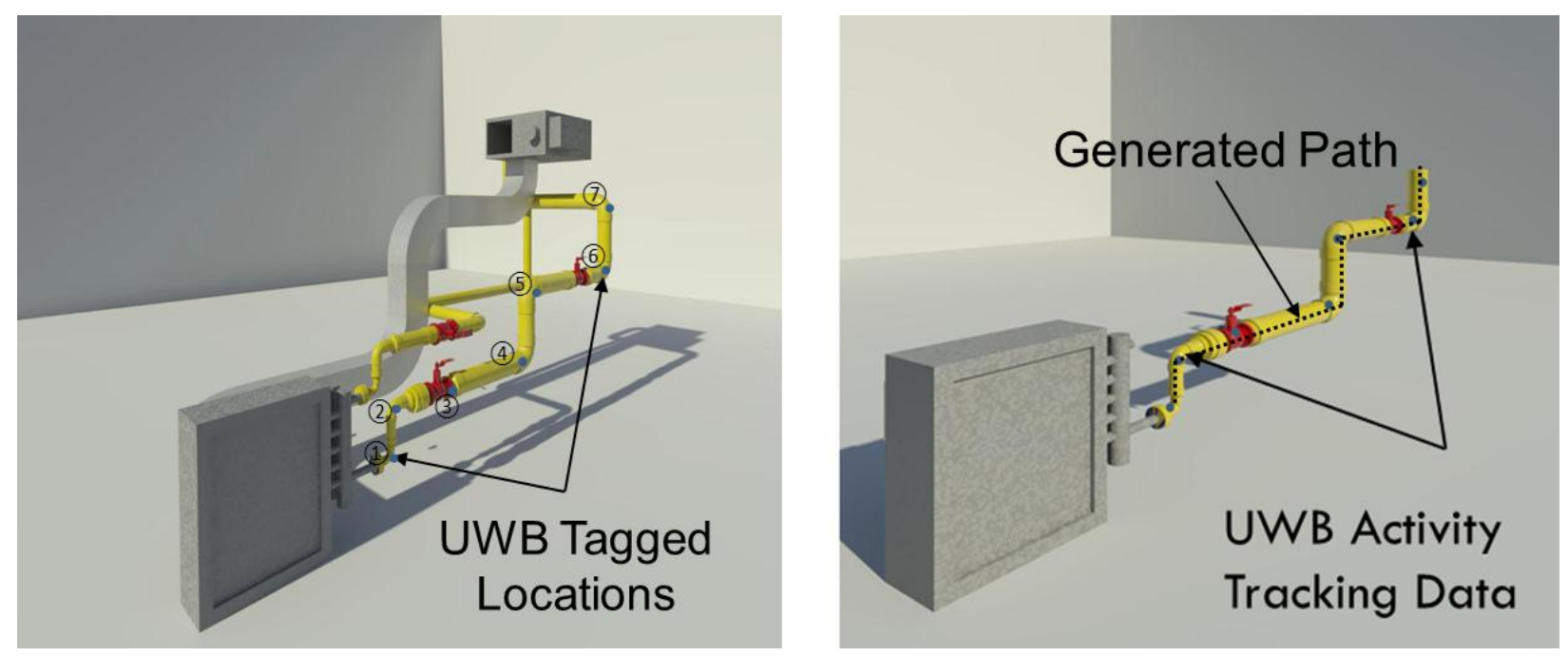

Figure 5-5: Schematics of the Activity-Tracking Data Capture

For the purposes of this research, a total station survey was used to measure the exact true coordinates of each point; however, for future implementations, these coordinates would be extracted directly from 3D CAD files. The next section explains how the activity-tracking data were captured and used in this research.

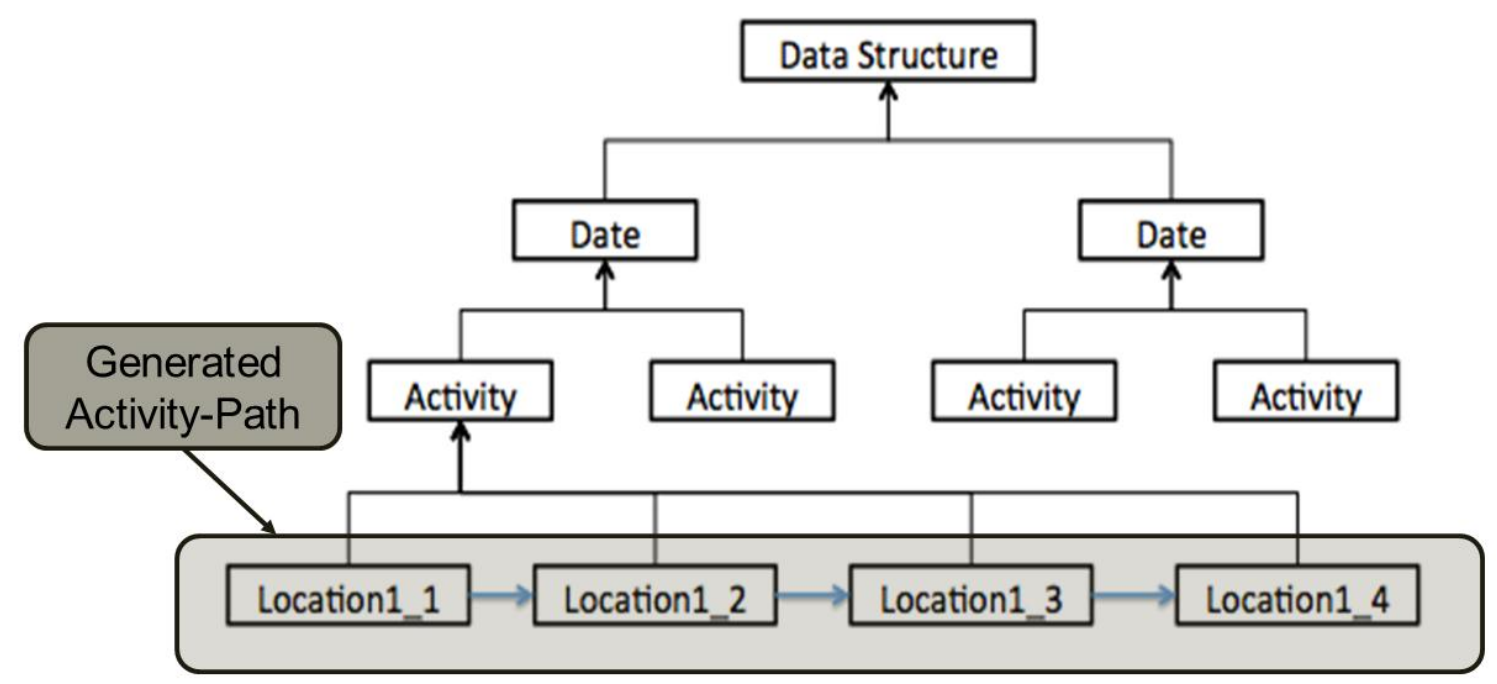

Figure 5-6: Hierarchical Structure of Activity-Based UWB Data 


\subsection{Field Collected Data vs. Simulations}

There were several factors affecting the data collection effort on this project. One of the main limitations was that the UWB implementation on this project was the first of its kind in an active construction environment, which posed a number of functionality related concerns as explained in Chapter 4. In terms of data collection, the efforts were limited to the fifth floor corridor of the building, imposed by site restrictions as well as equipment limitations. However, the piping network of the building ran vertically from various labs into the corridor of each floor and then up through the corridors to the penthouse equipment centre. Figure 5-7 shows the schematic elevation layout of the piping network of the building.

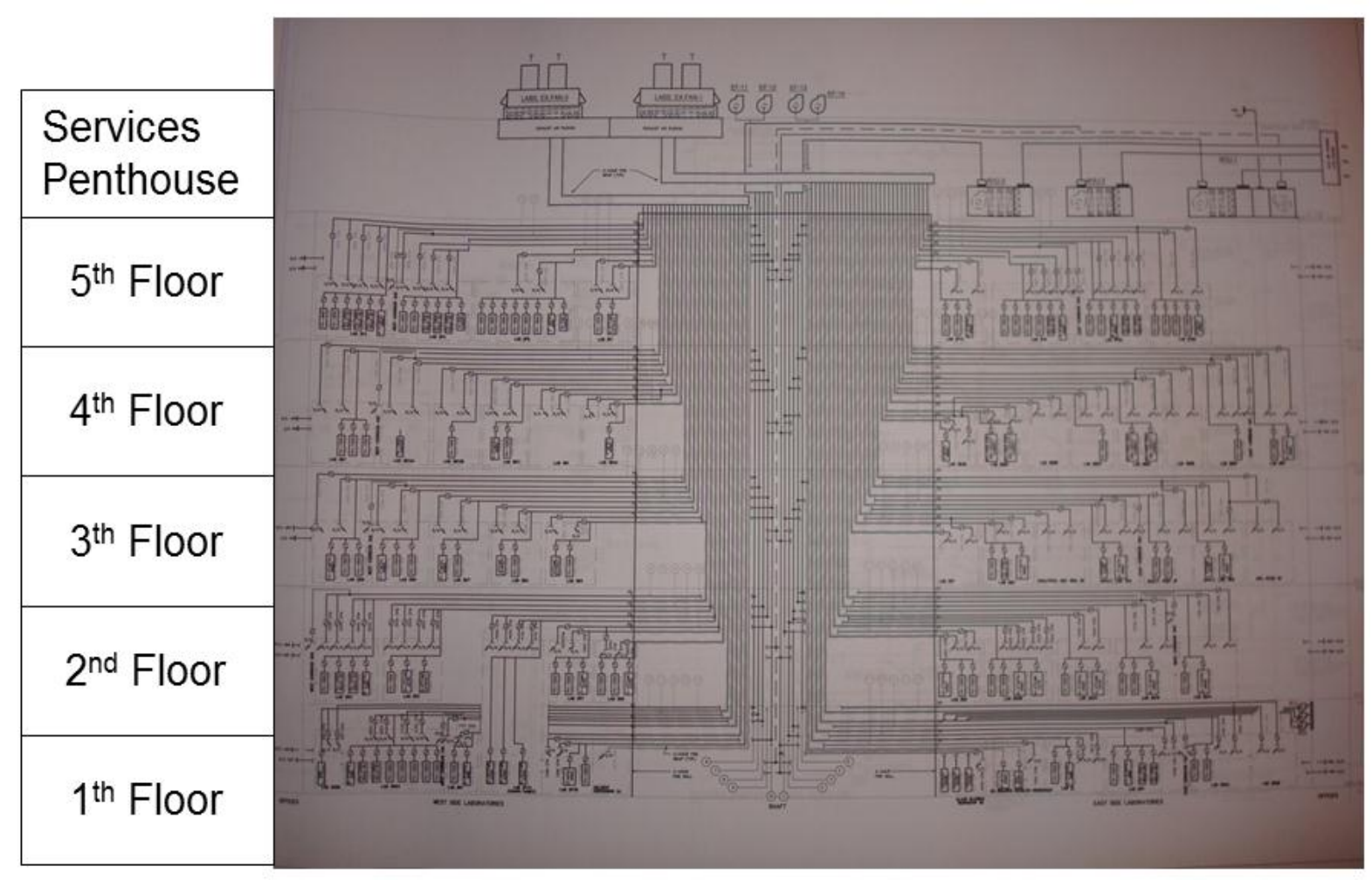

Figure 5-7: Elevation View of the Piping Network for Engineering VI Building

Due to the unique layout of the piping systems and the limitations of the data collection effort, only parts of the pipes for each system could be monitored in the fifth floor corridor where the UWB system was installed. In other words, only the pipe segments travelling vertically up from the fourth level corridor into the fifth level corridor could be tracked. The pipes entering the fifth 
floor corridor from the fifth floor labs entered the corridor at the ceiling level and directly entered the penthouse level and therefore could not be tracked effectively.

As the pipes and ducts entered the fifth level corridor from the lower level, they were permanently labeled at one to three points with point locators, as shown in Figure 5-8. At each data collection day, UWB tags were temporarily attached to these point locators, their location was recorded, and then the tags were removed. Therefore, for each point locator, there were a number of readings spread over the duration of the data collection program. Of course, the point locators associated with pipes that were installed first had more readings since they were present in a larger number of data collection days. These point locators were also surveyed using a total station, which measured the true location of the centre of each point locator, where the UWB tags were temporary attached during the data collection process. The true locations were then compared to the measured locations recorded by the UWB positioning system.
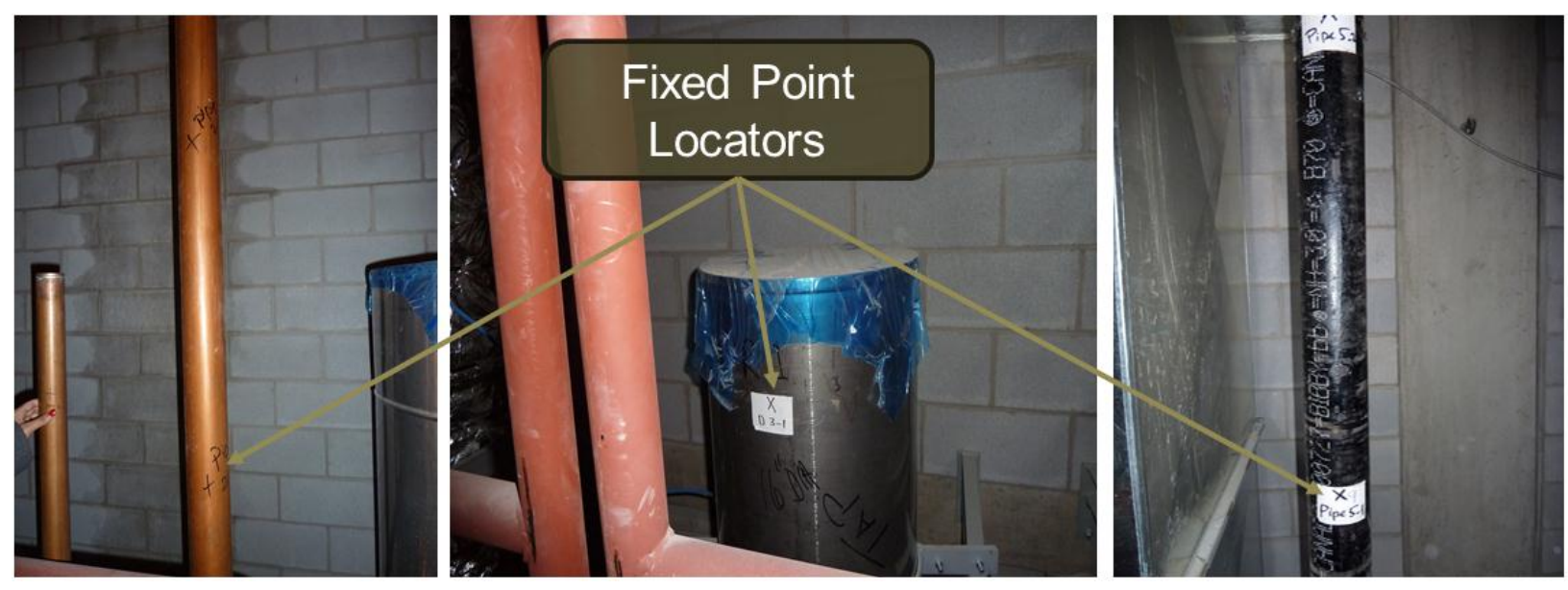

Figure 5-8: Point Locator Installations

The point readings that were measured throughout the data collection effort on the fifth floor corridor of this building were used in a number of ways for the development and partial validation of the developed model. The simplest deployment of the collected data was within the UWB performance deterioration investigation that was presented in detail in section 4.2 of this thesis. In that study, the repetitive redundancy of the readings for each point was leveraged to calculate the performance deterioration of the UWB positioning system as the site became more 
congested and the line of site was blocked. The more sophisticated deployments of the same data sets included the following two categories of simulated events:

1) As explained earlier in this section, each individual pipe line was only within the fifth floor corridor as it travelled vertically from the corridor on the lower floor to the penthouse floor. The height of the fifth floor corridor was deemed insufficient for any meaningful tracking of piping activities, both due to the short distance of installed pipes and also because of the uni-axis travel direction of pipelines (travelling straight up from lower corridor to penthouse). Therefore, a number of "simulated lines and paths" were generated using the recorded locator point readings. First, four "pipe-lines" were simulated by virtually connecting 5 to 10 point locators for each line, resulting in pipeline lengths ranging from 10 to $20 \mathrm{~m}$, as shown in Figure 5-9. A sample generated pipeline is illustrated in Figure 5-10 with a dashed line. Figure 5-11 illustrates the simulated pipeline that was generated using the point locators described in the above figures.

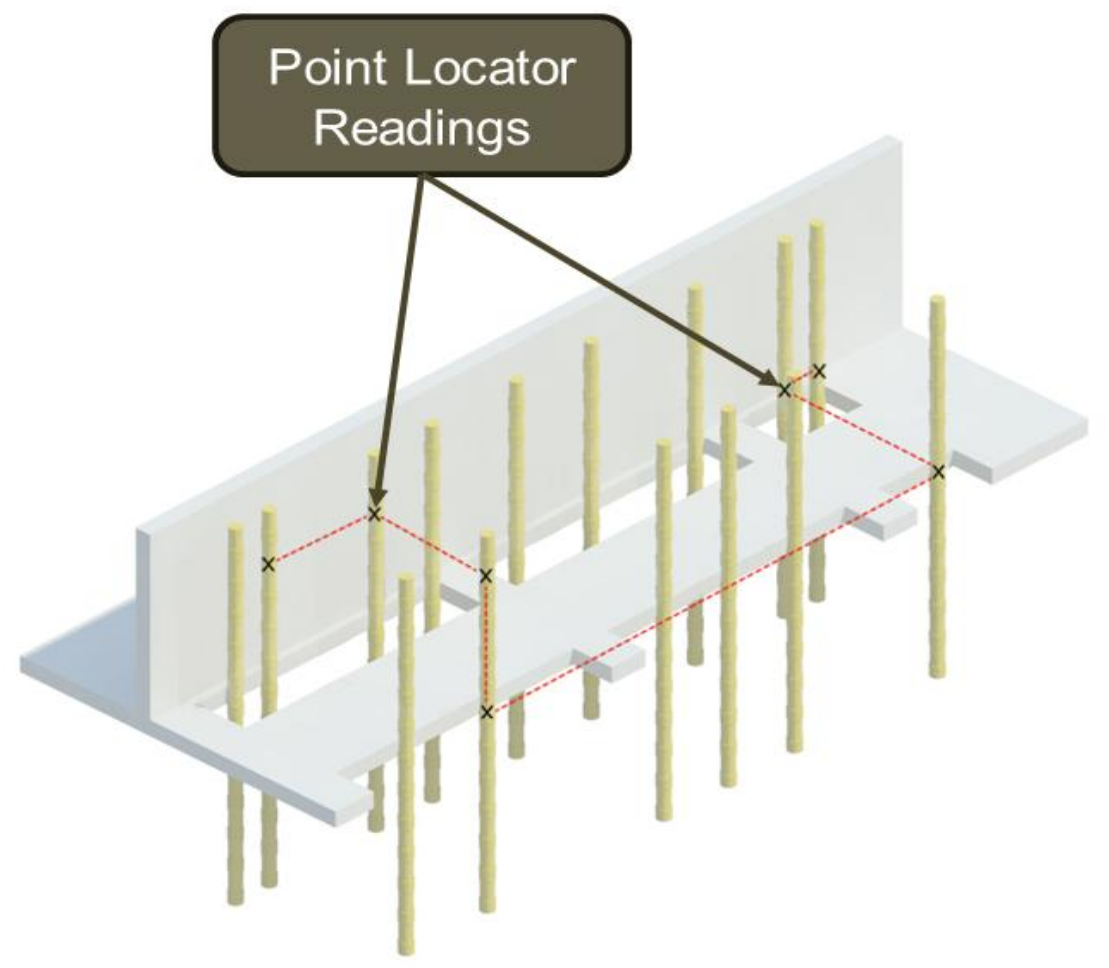

Figure 5-9: Connected Point Locator Readings 


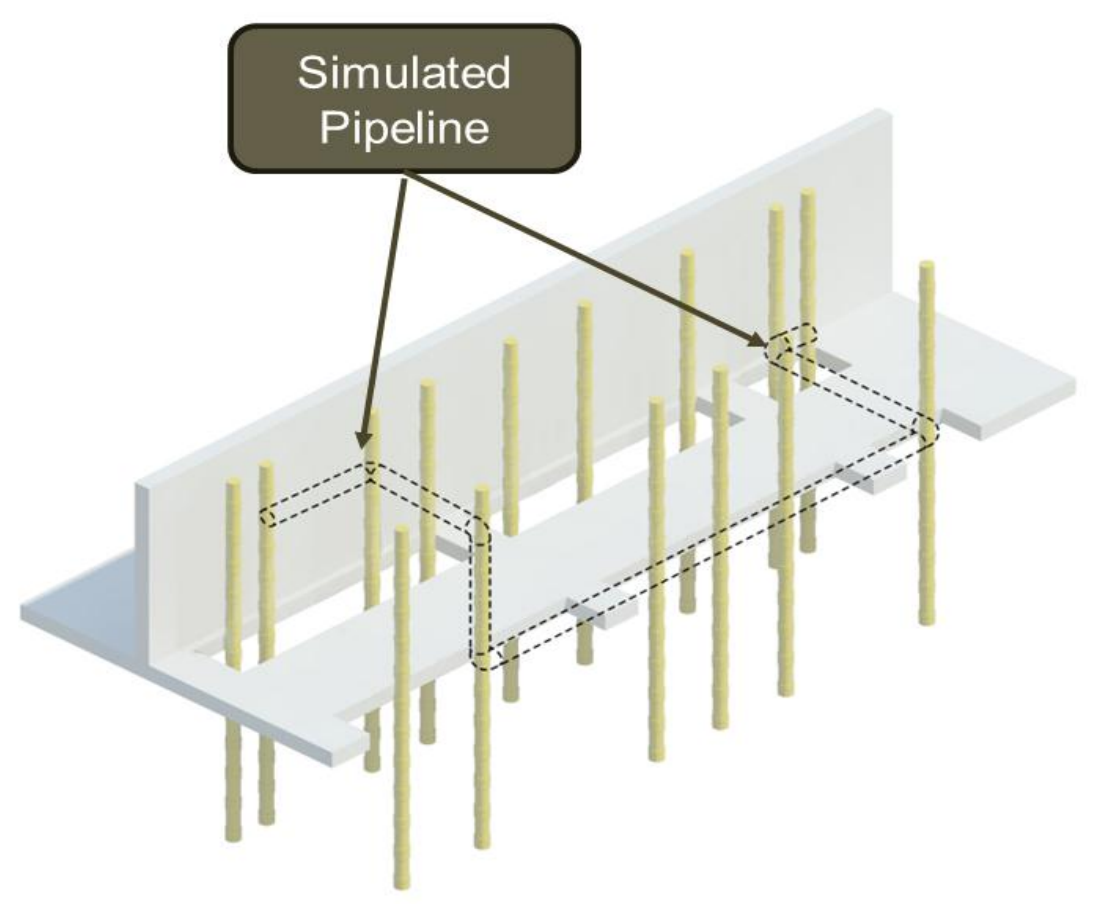

Figure 5-10: Virtually Generated Pipeline

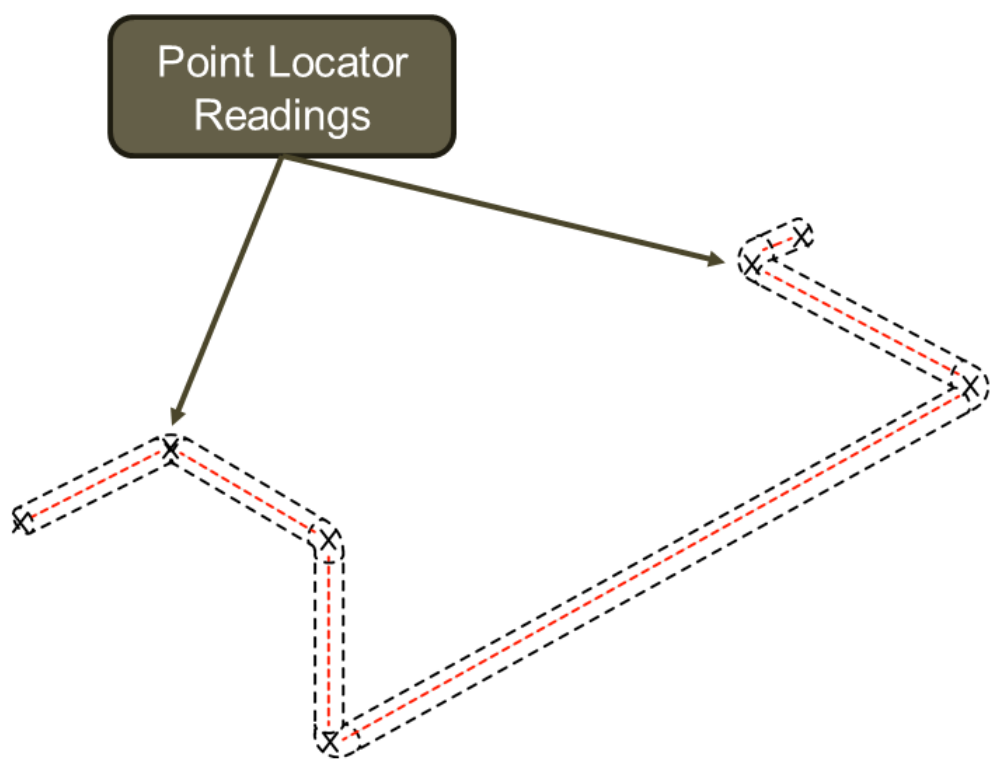

Figure 5-11: Simulated Pipeline as Used in the Model 
The distance between any two point locators was divided into two pipe spools, resulting in different-sized spools in the line. These generated pipelines were therefore nonexistent, but there were multiple site collected readings for a number of distinct points on the lines that matched the point locator readings. Then for each day's worth of data collected, the point locator readings were selectively chosen to indicate simulated installation, welding, or inspection activities. For example, for September $19^{\text {th }}$ reading, 4 out of 5 point locator readings could be used as the indicator-tag readings for the installation activity, while for October $14^{\text {th }}$ readings, 3 out of 5 point locator readings could be used for welding activity. The connected path between these points, was then used as the "generated path" for a given activity at a specific date. This type of simulation results in a number of consequences as explained at the end of this section.

2) Another category of simulated events using the site collected data was with respect to the material tracking component of the developed model. The material tracking engines within the model assume that UWB tags are attached to the pipe-spools as they enter the site and throughout the installation activity. Due to the piping layout, site restrictions, and equipment limitations presented above, the permanent attachment of tags to the pipes prior to installation of the pipes was not feasible for this project. Therefore, the first UWB reading recorded on each point locator was assumed to be the material tracking signal for that piece of pipe. For example, if the first UWB reading on pipe 8-1 was recorded on October $14^{\text {th }}$, it was assumed that pipe segment 8-1 was installed on October $14^{\text {th }}$, which was a relatively accurate assumption as the site was monitored 3 to 4 times a week during the data collection program. The readings taken on the following dates on point 8-1 were used for signalling the simulated welding and inspection activities for the generated pipelines as explained previously.

The final simulated or assumed input of the model was with respect to the object recognition results for volumetric site information. A number of existing object-recognition scripts are now able to automatically extract objects from the 3D point clouds obtained from 3D laser scanners. For the purposes of this research, Bosche's method (Bosche \& Haas, 2008) was chosen for the automated object recognition of the data from the 3D laser scanners. This method takes advantage of 3D CAD models as a-priori information and is able to provide accuracy levels of 
up to $100 \%$, in the case of steel structures (Bosche et al., 2008). The reported accuracy levels refer only to objects that have been installed at the exact location where they were planned to be installed, with very minimal tolerance values. Turkan et al. (2011) improved on Bosche's method and was able to calibrate it to enable automatic recognition of the elements of a concrete structure that had been captured in 3D laser scans. Turkan et al. used a fourdimensional BIM model as a-priori information for the object-recognition algorithm (Turkan, Bosche, Haas, \& Haas, 2011). At the time this research was being conducted, Guillemet was engaged in improving on Bosche's methodology, with the goal of automatically extracting piping objects from 3D laser scans, using a 3D CAD model as a-priori information.

The output from all of these variations on the object recognition scripts first developed by Bosche is consistent in format as they all produce binary detection values for each object in the CAD model. Analyzing object-recognition algorithms and calibrating them for the purposes of this study were beyond the scope of this research, so the output data were generated manually based on the documented performance of the fusion algorithms in other applications. Due to the technological limitations for object recognition of pipelines from point clouds, the fact that the pipelines were simulated in this research and that there were no physical spools and pipelines to be picked up by the 3D laser scanner, was irrelevant to the development of the model, as the object recognition results needed to be generated manually anyway.

Although field validation is always preferable over validation with partially simulated scenarios, the above simulations were necessary for the development and validation of the model presented in this thesis. The main factor that necessitated the replacement of a full field implementation of the model with the above simulated scenarios was that the components of the data fusion and management system needed to be developed up to the stage presented in this thesis before a complete field implementation could be justified and successfully implemented. The piping layout, site restrictions, equipment limitations, and technology limitations were the other factors that dictated the simulated validations of the model. A full implementation and final calibration of the model is presented as a recommendation for future work in this field, as the technologies required for this research become available and functional in the next few years. 


\subsection{Data Fusion Model and Results}

This section summarizes the data fusion processes used in this research in terms of the workflows for the data fusion problem as a whole and for the particular field implementation application for tracking piping progress. The use of workflows to model the fusion process for this research is an attempt to establish industry-wide standard workflows and procedures for data fusion, particularly with respect to applications for automated construction progress tracking. A number of workflow engines are used for construction industry applications. For this research, the Skelta BPM (Business Process Management) solution was used in order to demonstrate the use of workflows for data fusion for automated construction progress tracking. Although the iconography of the Skelta BPM system was adopted for the workflows presented in this thesis and the processes they represent were followed, the actual fusion algorithms were implemented in a MATLAB environment due to licensing and access considerations. Once the workflows have been accepted by the stakeholders in the construction industry, their implementation can be based on any programming environment. A step-by-step guide describing various components of the developed software is presented in Appendix $A$ and the entire MATLAB code, including all algorithms used in the model, is provided in Appendix $B$. The entire software, including Graphical User Interfaces (GUIs), fusion algorithms, and various functionalities were coded and programmed in MATLAB environment.

Figure 5-12 summarizes the iconography that was used in this research to represent the items and actions within the workflows. In this context, "Site Data Collection Point" refers to the data that were captured directly on the construction site, such as UWB readings and 3D laser scans. "Data provided by third party" indicates an additional category of input information, which was not collected directly on the site. For the purposes of this research, all information and data provided by the contractors and the owner were included in this category, such as the 3D model, the schedule, and requests for payment. "Final or Intermediary Output" refers to the results of the analysis and the fusion processes that are completed within the model. These could be the results of one of the sub-processes in the model or one part of the final output. "User Input" indicates occasions when manual user input is required for the process to continue. Users could be higher management, field technicians, etc., depending on the particular circumstances and as indicated in the workflows presented in this thesis. 

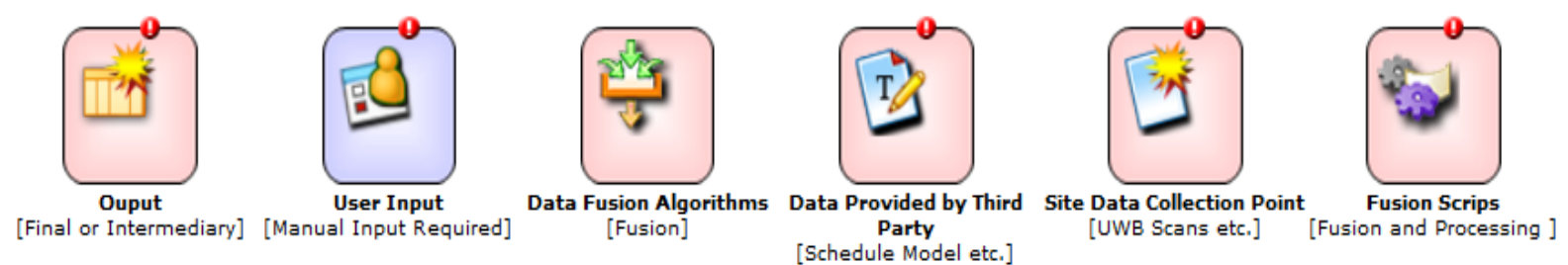

Figure 5-12: Workflow Iconography Legend

Finally, "Data Fusion Algorithms" are the fusion processes and algorithms that are used primarily to combine data from a variety of sources. These fusion processes are differentiated from "Scripts," which are algorithms that, in addition to potentially fusing data from different sources, also manipulate, process, and analyse the data for a number of applications. These two processes are usually complementary and can be grouped together into one main process called "Fusion and Analysis"; however, in this research, they were differentiated in order to provide greater resolution in the representation of the model. Figure 5-13 shows the overall architecture of the activity-based data fusion model presented in this research, which is suitable for use as an industry standard workflow and process architecture for the development of automated progress tracking applications for construction projects.

In this model, the main components of the input to the system are the automated material tracking (UWB), Activity Progress Tracking (UWB), and object recognition results (from 3D point clouds). The position estimation system is established using UWB and is part of both the material- and the activity-tracking processes. The information from these site-collected data is then fused in the model with the information from the 3D CAD or BIM model of the site. After the fusion process, the progress of the activities can be estimated. The activity-based fusion process also enables the efficient utilization of a wide range of applications, such as earned value estimates and automated schedule updating. These applications are performed as part of the "Project Control Applications" stage, at which point, administrative documents such as the schedule and requests for payment are introduced into the fusion process.

The workflow represented in Figure 5-13 can be modified and customized, depending on the type of project and on the level of granularity required in the process description. Figure 5-14 illustrates the detailed architecture of the fusion model for the specific application of the 
automated progress tracking of piping and HVAC ducts for an industrial-type project, which is the focus of the research presented in this thesis.

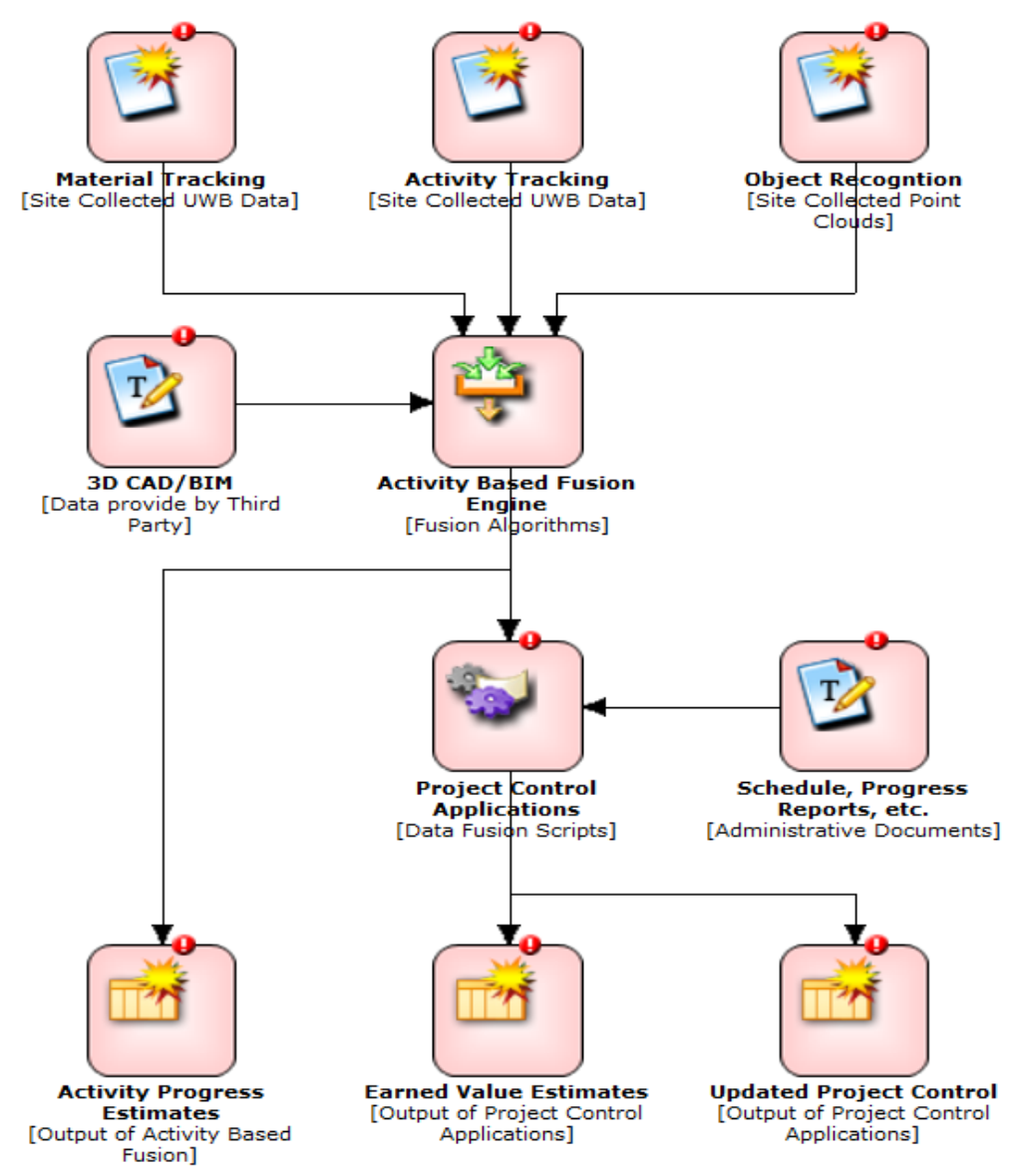

Figure 5-13: Activity-Based Fusion Architecture

The remainder of this chapter is structured according to the section numbers displayed under the fusion processes in Figure 5-14. After a brief discussion of the UWB data collected on site, in section 5.5.1, the five fusion processes of Material-Tracking Fusion, Object Recognition Script, Path Generation for Indicator Tag Script, Activity-Based Fusion, and Project Control Applications, are presented in sections 5.5.2 to 5.5.6, respectively, according to the section numbers indicated in Figure 5-14. 


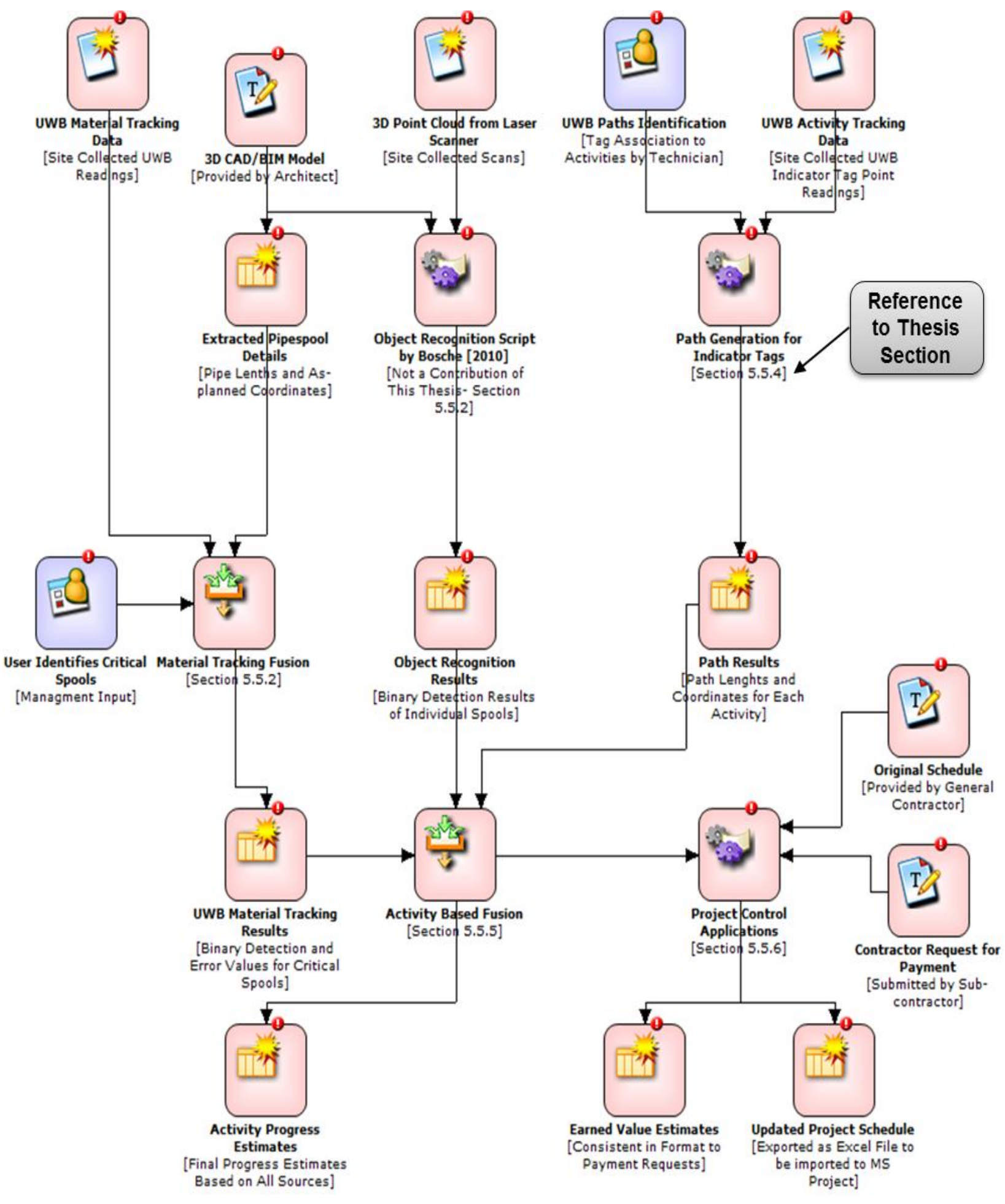

Figure 5-14: Workflow for Site Implementation Research 


\subsubsection{Ultra-Wideband (UWB) Positioning System}

An UWB positioning system was used both as the material-tracking engine of this model and its activity-tracking tool. UWB tags were placed for 30 seconds to one minute at specific locations, and the readings were recorded with a frequency of 4 to 8 readings per second. The UWB data at each measurement location and instance consisted of text files containing a scatter of threedimensional points, as defined by a global coordinate system. The UWB system applied in this research consisted of active UWB tags and receivers manufactured by the Ubisense ${ }^{\circledR}$ Company.

An UWB file may contain readings from multiple tags, with each tag representing a different location. Each UWB tag is also accompanied by an unique ID number that is extracted when the data are imported. If a tag ID is linked to an object (e.g. pipe spool), the raw data can be separated appropriately and then categorized within the data fusion engine for further processing and use. Figure 5-15 shows a screen capture of one UWB input file, in which each row represents a separate reading. The tag ID, 3D coordinates, and time stamp are identified in this figure. The other values shown in this figure were not used in the current research. Depending on the duration of the data capture at any point and the frequency of the data recording, each data file may contain hundreds, or sometimes thousands of readings, often from a variety of tags.

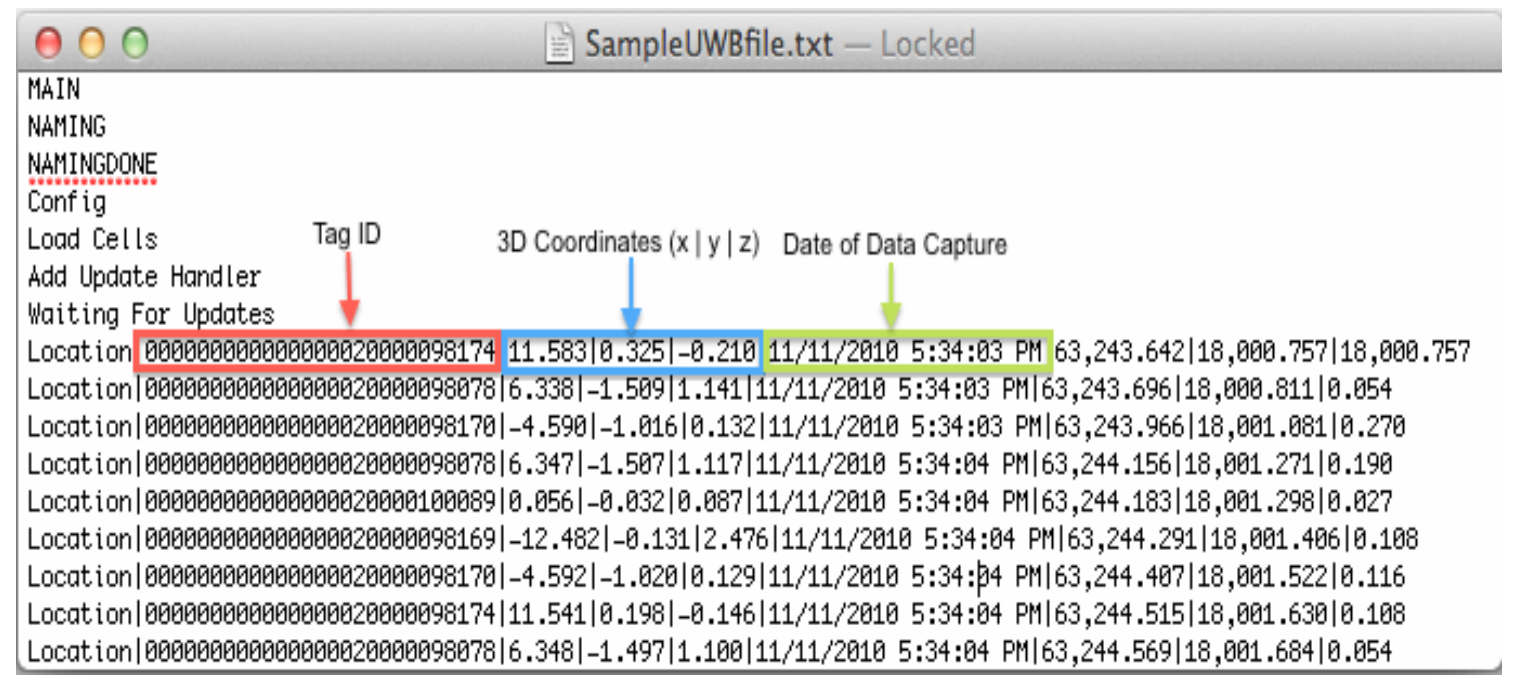

Figure 5-15: Example of UWB Data Input 
Figure 5-16 shows a sample UWB file coordinate scatter plotted on a three-dimensional plane; the scatter is illustrated by the black dots, in comparison with the true location of the measured point, as indicated by the black triangle.

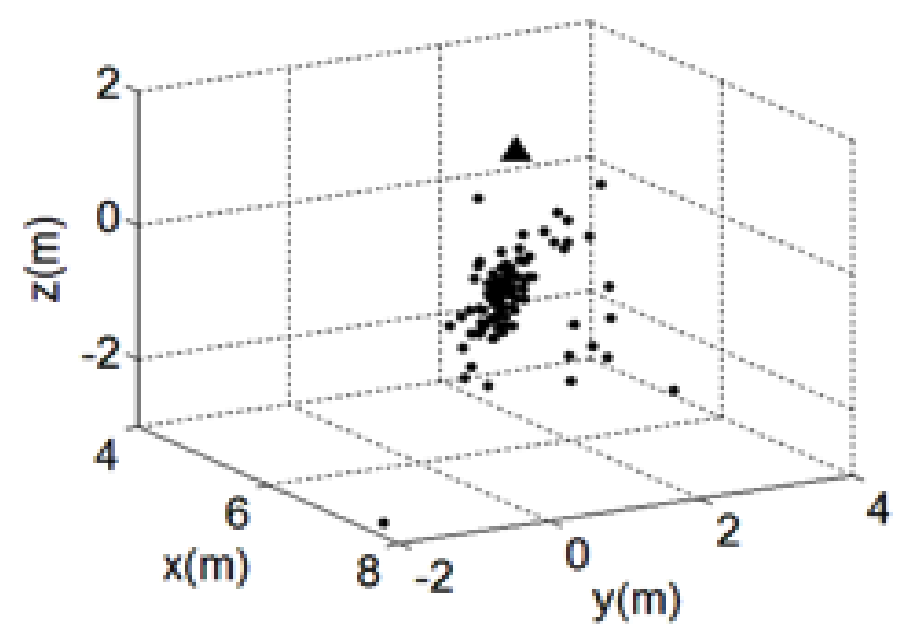

Figure 5-16: 3D Scatter of UWB Readings for One Point

In this thesis, because the tags are used to mark pipe, valve, and joint locations, UWB location error values, or simply UWB error values, are defined as the linear three-dimensional distance between the average coordinates of the scatter and the "as planned" coordinates of the point where the object should have been installed. These error values are used throughout the fusion processes for detection and calibration purposes. For the purposes of the data analysis required for this research, the "true location" of the tag was measured using a total station survey. Since the performance of the UWB positioning system in active and congested construction environments was unknown, this information was required in order to measure the possible deterioration in measurement accuracy during the life of the project as the construction environment evolved. However, in future implementations where this model is fully implemented, the errors will be calculated as the distance between the average of the scatter and the "as planned" point that was extracted from the 3D model, which will be the assumed true location of the tag, and the error value will only be used for detection purpose. With this explanation of the UWB data collection and data processing procedure as background, the fusion processes are described in the following sections. 


\subsubsection{Material-Tracking Fusion}

Figure 5-17 highlights the Material-Tracking Fusion engine implemented as code in MATLAB, also shown in Figure 5-14. Three data sources are used as input for the material-tracking fusion engine: the raw UWB material-tracking data (3D position data), the user-identified list of critical spools, and the pipe spool details (3D locations and lengths) extracted from the 3D CAD or BIM model. The output of this fusion engine is the material-tracking results (detection results and the calculated error values for critical spools), which are then used for activity-based fusion algorithms.

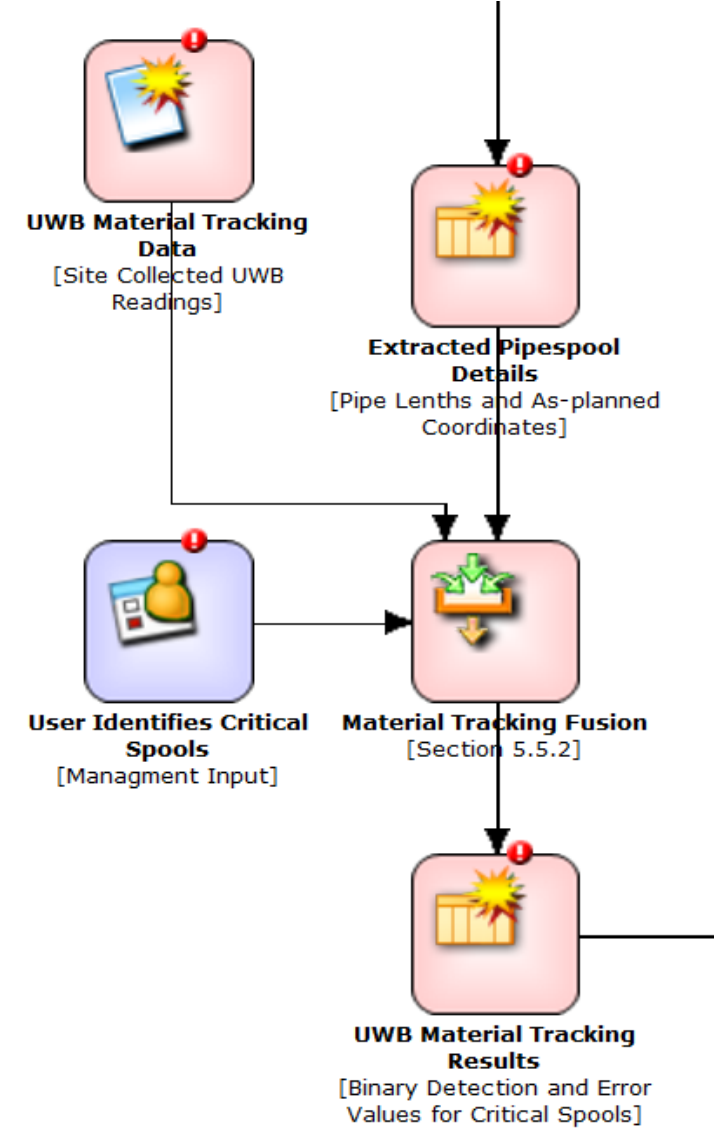

Figure 5-17: Material-Tracking Fusion

Two types of error may be present with respect to the automatic detection of objects: Type I and Type II. It should be noted that, for the purposes of this research, the definitions of these errors 
are the ones presented in Table 5-1, which may differ from the definitions of errors in the context of hypothesis testing. In this research, a Type I error refers to the detection of a nonexistent object, or a "false positive" error, and a Type II error refers to the failure to detect an existing object, or a "false negative" error.

Table 5-1: Definition of Type I and Type II Errors

\begin{tabular}{ccc}
\hline & Detected & Not Detected \\
\hline Existent & OK & Type II Error \\
Nonexistent & Type I Error & OK \\
\hline
\end{tabular}

Due to the inherent characteristics and capabilities of the technologies and the experimental regime used in this research, a Type I error was associated only with object recognition results from the 3D laser scanner, while a Type II error could be present in both 3D scanner and UWB results. Since only the presence of UWB tags was detected by the receivers, no tags were expected to be detected if the object was nonexistent. Special cases, in which the tags may be accidently removed from items or human errors in identifying a wrong pipe, are not considered as part of the error of this fusion engine and are therefore not considered in this error classification. A description of the input variables, the fusion algorithms, and the output of the material tracking fusion engine is presented in the following subsections.

\subsubsection{Input Sources for the Material-Tracking Engine}

Material tracking can be used as a tool for assessing the progress of all activities that can be linked to physical objects. In the case of piping projects, material tracking is linked directly to delivery and installation activities and feeds indirectly into welding and inspection activities. In many projects, tracking each individual piece of equipment or material is unnecessary. For example, in piping projects, a large number of generic-sized or generic-type spools may not need to be tracked, but a limited number of specially ordered spools may require close tracking and monitoring because misplacing them or not having them delivered on schedule could represent a significant risk and liability for the project. Simulating a case that involves such specially ordered spools therefore requires user input in order to identify which spools need to be tracked. In other cases, such as the project used for the implementation of this model, critical 
spools were strategically chosen based either on the location where they were to be installed or on the system they belonged to for progress tracking purposes only. It was critical that only unique spools or connectors be marked and tagged with UWB tags, so that their expected installed location could be known a priori using the 3D CAD or BIM model. The "Extracted Pipespool Details" section, as identified in the workflow diagram shown in Figure 5-14, refers to the details about the spools, including their type, length, diameter, and expected or as-planned 3D coordinates.

With recent developments in supply chain management research, it is now preferred that all critical items be tracked at the fabrication stage. If critical items are tagged with active GPS receivers, their status can be tracked even if they travel around the world. These tags can then be replaced with corresponding UWB tags after they enter a laydown yard. Alternatively, and if live tracking outside of the site is not required, the fabricator can place UWB tags at the fabrication shop. The tag ID can then be uploaded into the BIM model by the fabricator and extracted as part of the "Extracted Pipe-spool Details" in the current fusion model. For the purposes of this research, the tags needed to be installed only after the spools had entered the site. The next section presents the fusion algorithms and processes that were employed within the Material-Tracking Fusion engine.

\subsubsection{Material-Tracking Fusion Algorithms}

This engine is able to import and process both spreadsheets and text files, and also offers a graphical user interface (GUI) that allows simple, user-friendly interaction. Every process within the workflow, including the acquisition of user-input data, is accompanied by pop-up instructions that guide the user. This system was applied consistently throughout all components of the fusion model architecture illustrated in Figure 5-14.

To successfully process UWB material-tracking data, an Excel file containing the spool identification numbers and the as-planned coordinates from the 3D CAD model, as shown in Figure 5-18, was imported into the fusion software. This figure shows only the spools for line 1 and part of the spools for line 2. Without this spreadsheet, an UWB location error value could not be calculated, making this file crucial to the material-tracking process. The entire spreadsheet is analyzed, and the spool identification numbers and their coordinates are stored 
in an indexed array. In future implementations, the information on this figure would be extracted directly from a 3D CAD or a BIM model.

\begin{tabular}{|c|c|c|c|c|c|c|c|c|c|c|c|}
\hline & $\mathbf{A}$ & B & C & D & E & $F$ & G & H & I & J & $K$ \\
\hline 1 & Spool & $\mathrm{x} 1$ & $\mathrm{y} 1$ & 21 & $\times 2$ & $y 2$ & 22 & xmid & ymid & zmid & Length \\
\hline 2 & Spool1_1 & -3.952 & -1.649 & 1.607 & -0.693 & -0.015 & 1.606 & -2.322 & -0.832 & 1.607 & 3.645 \\
\hline 3 & Spool1_2 & -0.693 & -0.015 & 1.606 & 2.565 & 1.619 & 1.605 & 0.936 & 0.802 & 1.606 & 3.645 \\
\hline 4 & Spool1_3 & 2.592 & 1.614 & 1.517 & 1.371 & 0.854 & 0.803 & 1.981 & 1.234 & 1.160 & 1.606 \\
\hline 5 & Spool1_4 & 1.371 & 0.854 & 0.803 & 0.150 & 0.094 & 0.088 & 0.761 & 0.474 & 0.445 & 1.606 \\
\hline 6 & Spool1_5 & 0.198 & 0.001 & 0.000 & 4.111 & 0.022 & 0.002 & 2.154 & 0.012 & 0.001 & 3.914 \\
\hline 7 & Spool1_6 & 4.111 & 0.022 & 0.002 & 8.025 & 0.044 & 0.003 & 6.068 & 0.033 & 0.002 & 3.914 \\
\hline 8 & Spool1_7 & 8.420 & 0.042 & 0.003 & 9.789 & 0.023 & -0.001 & 9.104 & 0.032 & 0.001 & 1.369 \\
\hline 9 & Spool1_8 & 9.789 & 0.023 & -0.001 & 11.158 & 0.003 & -0.005 & 10.473 & 0.013 & -0.003 & 1.369 \\
\hline 10 & Spool1_9 & 11.159 & -0.008 & 0.010 & 3.831 & -0.345 & 0.597 & 7.495 & -0.177 & 0.304 & 7.359 \\
\hline 11 & Spool1_10 & 3.831 & -0.345 & 0.597 & -3.497 & -0.682 & 1.185 & 0.167 & -0.513 & 0.891 & 7.359 \\
\hline 12 & Spool2_1 & -4.641 & 0.045 & 0.001 & -2.759 & -0.216 & 0.502 & -3.700 & -0.086 & 0.252 & 1.965 \\
\hline 13 & Spool2_2 & -2.759 & -0.216 & 0.502 & -0.876 & -0.477 & 1.004 & -1.817 & -0.347 & 0.753 & 1.965 \\
\hline 14 & Spool2_3 & -0.322 & -0.521 & 1.095 & 3.657 & -0.602 & 1.370 & 1.668 & -0.561 & 1.233 & 3.989 \\
\hline 15 & Spool2_4 & 3.657 & -0.602 & 1.370 & 7.635 & -0.683 & 1.645 & 5.646 & -0.642 & 1.508 & 3.989 \\
\hline 16 & Spool2_5 & 7.637 & -0.654 & 1.655 & 2.224 & 0.005 & 1.467 & 4.930 & -0.325 & 1.561 & 5.456 \\
\hline 17 & Spool2_6 & 2.224 & 0.005 & 1.467 & -3.189 & 0.664 & 1.278 & -0.483 & 0.334 & 1.372 & 5.456 \\
\hline
\end{tabular}

Figure 5-18: As-Planned Coordinates Extracted for the Pipeline

Based on the imported Excel spreadsheet, the user is prompted to select the pipeline to be tracked and to specify the critical spools. In future implementations, the selection of "pipeline" can be changed to selection of "work packages," "value packages," etc., depending on the particular application. The user must then select the relevant UWB material-tracking data files. In the current state of the software, this step is performed manually because each pipeline has more than one set of files since multiple readings were taken of the same state of the project for the purposes of verification and validation. However, for implementation on future projects, the uploading of the files can be fully automated based on a prescribed naming convention that would enable the code to relate the files to their respective pipelines.

The UWB material-tracking files are processed individually in a sequential loop. For each file, part of the filename string is matched with a specific critical spool ID from the user-defined list and is then extracted onto an array variable. The UWB location error value is then calculated. A Boolean value indicating the detection of the spool within the site is also generated, and both the error and the detection parameters are stored within the array, as indicated by the function shown in Figure 5-19. The error value are used in Activity-Based Fusion engine to distinguish 
between installed correction location, installed wrong location, and delivered criteria, depending on how far the tag is located from the "as-planned" co-ordinates.

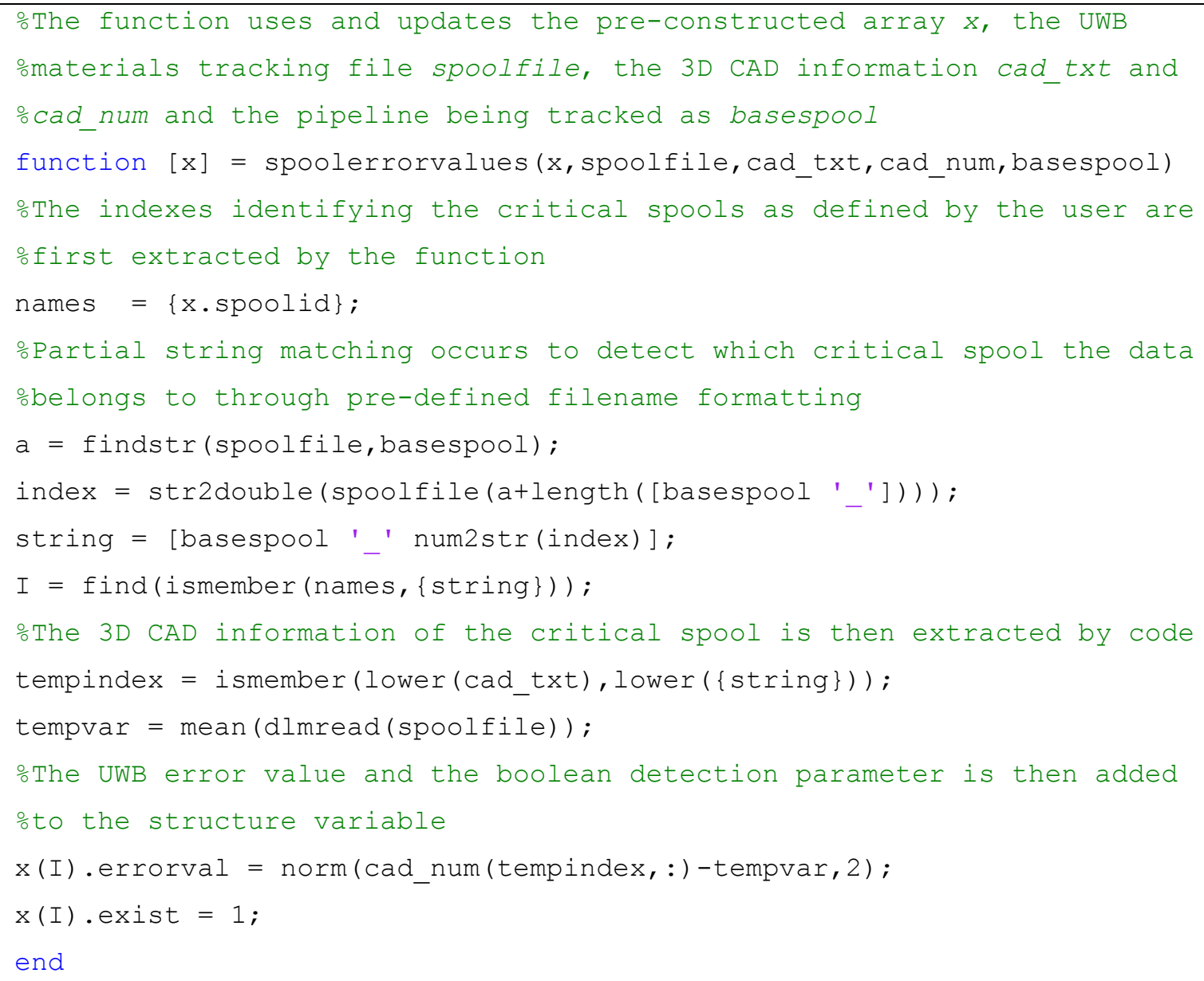

Figure 5-19: Function "Spoolerrorvalues" for Processing the Material-Tracking Files

\subsubsection{Object Recognition Script}

The second fusion engine in the fusion model, as shown in Figure 5-14, is the Object Recognition Script. In this research, Bosche's (2009) approach is recommended for automated object recognition from 3D point clouds. The details of his approach were presented in Section 2.3 of this thesis. The inputs of this script in the fusion model are the 3D CAD model and 3D laser point clouds and its output is a list of binary detection values ( 0 or 1 ) for every object in the CAD model. These binary values are carried forward to the next fusion engine of the model. 
The following sections provide a detailed explanation of the methods and algorithms that have been included in the fusion model to address the challenges inherent in the use of object recognition algorithms for piping activities. The use of these methods in the context of activitybased fusion is discussed in section 5.5.5. The next section describes the collection and analysis of the activity-tracking data, which is the final input used in the activity-based fusion processes.

\subsubsection{Path Generation for Indicator Tags}

The third engine of the model is called Path Generation for Indicator Tags, as shown in Figure $5-20$. This engine is one of the original and unique contributions of the research presented in this thesis. The data that were used in the algorithms of this engine are introduced previously in Sections 5.3 and 5.4 of this thesis.

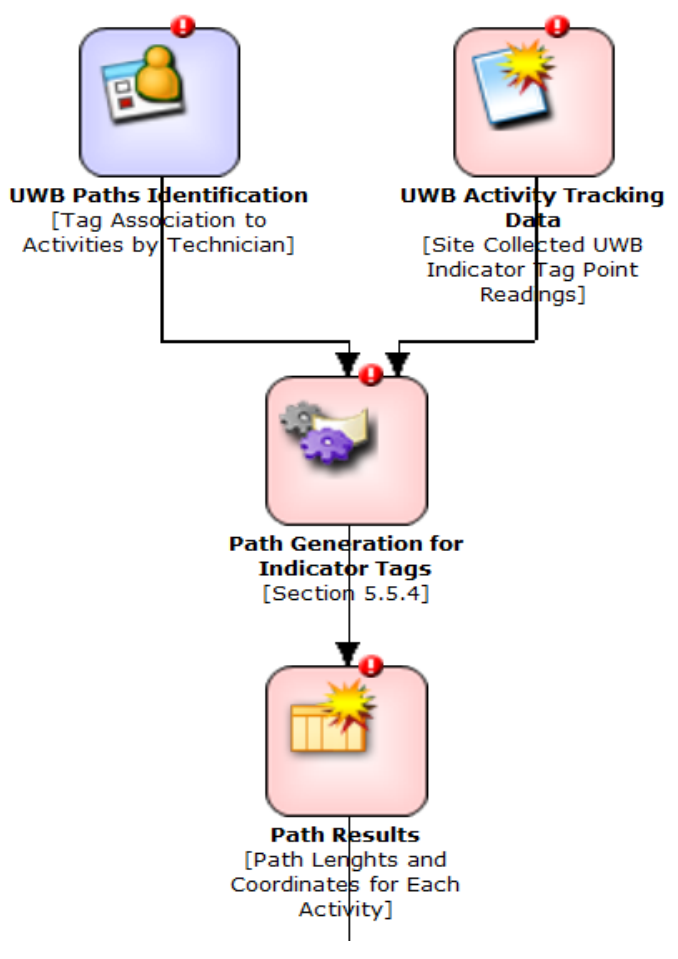

Figure 5-20: Path Generation for Indicator Tags

This engine uses the UWB activity-tracking data as well as UWB path identification input from the field technicians or inspectors to establish the activity paths. In the Path Generation function, 
a three-dimensional path is established between the average locations of sequentially defined UWB files. The files contain 3D scatters in the same way that the UWB material-tracking files do, but they differ in that the file name contains information about the sequence in which the readings were taken.

Figure 5-21 shows a snapshot of part of the activity-tracking UWB data folder files (left) and a sample open file showing the coordinates of a three-dimensional scatter (right). These data files have been filtered and modified from the raw format shown in Figure 5-15, by separating the data from each tag and organizing it according to the data structure explained previously.

\begin{tabular}{|c|c|}
\hline Earlier & $-0.577,-0.597,1.28$ \\
\hline nov9_insp_line2_2.txt & $-0.639,-0.632,1.269$ \\
\hline nov9_insp_line2_3.txt & $-0.62,-0.677,0.978$ \\
\hline nov9_insp_line4_1.txt & $\begin{array}{l}-0.63,-0.628,1.182 \\
-0.634,-0.666,1.201\end{array}$ \\
\hline nov9_insp_line 4 2.txt & $-0.613,-0.639,1.245$ \\
\hline H nov9 insp line 43 txt & $-0.607,-0.638,1.213$ \\
\hline 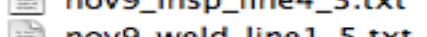 & $-0.628,-0.65,1.202$ \\
\hline 望 nov9_weld_line1_5.txt & $-0.637,-0.649,1.116$ \\
\hline 望 nov9_weld_line1_6.txt & $-0.635,-0.68,0.946$ \\
\hline nov9_weld_line2_4.txt & $-0.601,-0.676,0.919$ \\
\hline nov9_weld_line2_5.txt & $-0.611,-0.627,1.317$ \\
\hline nov9_weld_line3_1.txt & $-0.628,-0.672,0.979$ \\
\hline nov9_weld_line3_2.txt & $-0.64,-0.664,1.171$ \\
\hline nov9_weld_line3_4.txt & $-0.647,-0.682,1.095$ \\
\hline nov9_weld_line3_5.txt & 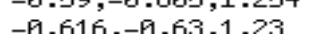 \\
\hline
\end{tabular}

\section{Figure 5-21: Snapshot of the File Format of the Activity-Tracking UWB Data}

In this research, a daily or even weekly progress estimate was deemed sufficient, which meant that the welders would not be required to carry tags all the time; instead, the simulations assumed that the progress of the welding activity was tagged once every couple of days. The same technique was used for the installation and inspection activities. This indicator-tag strategy for collecting data with respect to individual activities could be extended to include any other activities at a construction site, with minimal modifications to the structure of the fusion algorithms presented in this thesis.

For the engine to properly sort the raw data onto an array, a naming convention was established for all files as shown in Figure 5-21. The individual filenames carry the information regarding the date of data collection, the activity being tracked, the pipe path to which each file belongs, and the sequence of the readings. Based on the extracted sequence of location readings stored 
within the variable, a three-dimensional path is mathematically defined by linking the average of each point cloud to form a three-dimensional line. This process was presented in Section 5.3 and Figure 5-6. From the path, a vector containing each segment length and a parameter containing the total sum of the segments are calculated via a function calibrated to process the array depicted in Figure 5-6. The as-built path length from the UWB tagging is compared to the as-planned path length from the 3D CAD model, and an error for each path is calculated, as shown in Table 5-2. The results from the field experiments showed that the overall error for all paths combined was less than $2 \%$, calculated as the difference between the total length of all as-planned paths and the total length of all as-built paths.

Table 5-2: Generated Path Lengths and Errors

\begin{tabular}{cccccc}
\hline Date & $\begin{array}{c}\text { Line and } \\
\text { Activity }\end{array}$ & $\begin{array}{c}\text { Total Measured } \\
\text { Length }(\mathbf{m})\end{array}$ & $\begin{array}{c}\text { Total True } \\
\text { Length }(\mathbf{m})\end{array}$ & $\begin{array}{c}\text { Error } \\
(\mathbf{m})\end{array}$ & $\begin{array}{c}\text { Absolute Value } \\
\text { Error }(\mathbf{m})\end{array}$ \\
\hline Sep_21 & line1_inst & 6.97 & 7.49 & -0.51 & 0.51 \\
Sep_24 & line1_inst & 11.98 & 11.83 & 0.15 & 0.15 \\
Sep_29 & line1_inst & 3.24 & 3.13 & 0.10 & 0.10 \\
Sep_29 & line1_weld & 8.90 & 11.09 & -2.19 & 2.19 \\
Oct_01 & line1_weld & 3.28 & 3.13 & 0.15 & 0.15 \\
Oct_05 & line2_inst & 11.76 & 12.76 & -1.00 & 1.00 \\
Oct_07 & line3_inst & 14.10 & 13.72 & 0.38 & 0.38 \\
Oct_07 & line4_inst & 7.14 & 6.51 & 0.63 & 0.63 \\
Oct07 & line2_weld & 8.65 & 8.54 & 0.10 & 0.10 \\
Oct_19 & line1_inst & 15.49 & 14.92 & 0.57 & 0.57 \\
Oct_19 & line2_inst & 12.92 & 12.93 & -0.01 & 0.01 \\
Oct_19 & line3_inst & 15.22 & 15.97 & -0.75 & 0.75 \\
Oct_19 & line4_inst & 13.34 & 12.91 & 0.43 & 0.43 \\
Oct19 & line4_weld & 5.15 & 4.52 & 0.63 & 0.63 \\
Nov_09 & line2_insp & 8.65 & 8.54 & 0.10 & 0.10 \\
Nov_09 & line4_insp & 6.49 & 2.77 & 3.72 & 3.72 \\
Nov_09 & line1_weld & 15.20 & 14.92 & 0.28 & 0.28 \\
Nov_09 & line2_weld & 2.07 & 1.45 & 0.62 & 0.62 \\
Nov_09 & line3_weld & 18.58 & 18.46 & 0.12 & 0.12 \\
Nov_09 & line4_weld & 12.00 & 10.91 & 1.09 & 1.09 \\
Nov_11 & line1_insp & 18.45 & 18.05 & 0.41 & 0.41 \\
Nov_11 & line2_insp & 26.69 & 25.69 & 1.00 & 1.00 \\
Nov_11 & line3_insp & 17.15 & 18.46 & -1.31 & 1.31 \\
Nov_11 & line4_insp & 13.21 & 12.91 & 0.31 & 0.31 \\
\hline \multicolumn{2}{c}{ Total Sum } & $\mathbf{2 7 6 . 6 3}$ & $\mathbf{2 7 1 . 6 2}$ & $\mathbf{5 . 0 1}$ & $\mathbf{1 6 . 5 9}$ \\
\hline
\end{tabular}


If the absolute value of the error was considered for each path segment, in which case the positive and negative errors would not cancel each other out, the total error was approximately $6 \%$. These results demonstrate that most of the uncertainty associated with the 3D scatter readings has been cancellled out and has had a minimal impact on the overall results. The next section presents the fusion process for combining the input from the Material-Tracking Fusion, Object Recognition Script, and Path Generation for Indicator Tag processes.

\subsubsection{Activity-Based Fusion}

The results from the previous three fusion processes are fused and integrated in the ActivityBased Fusion engine, as shown in Figure 5-22.

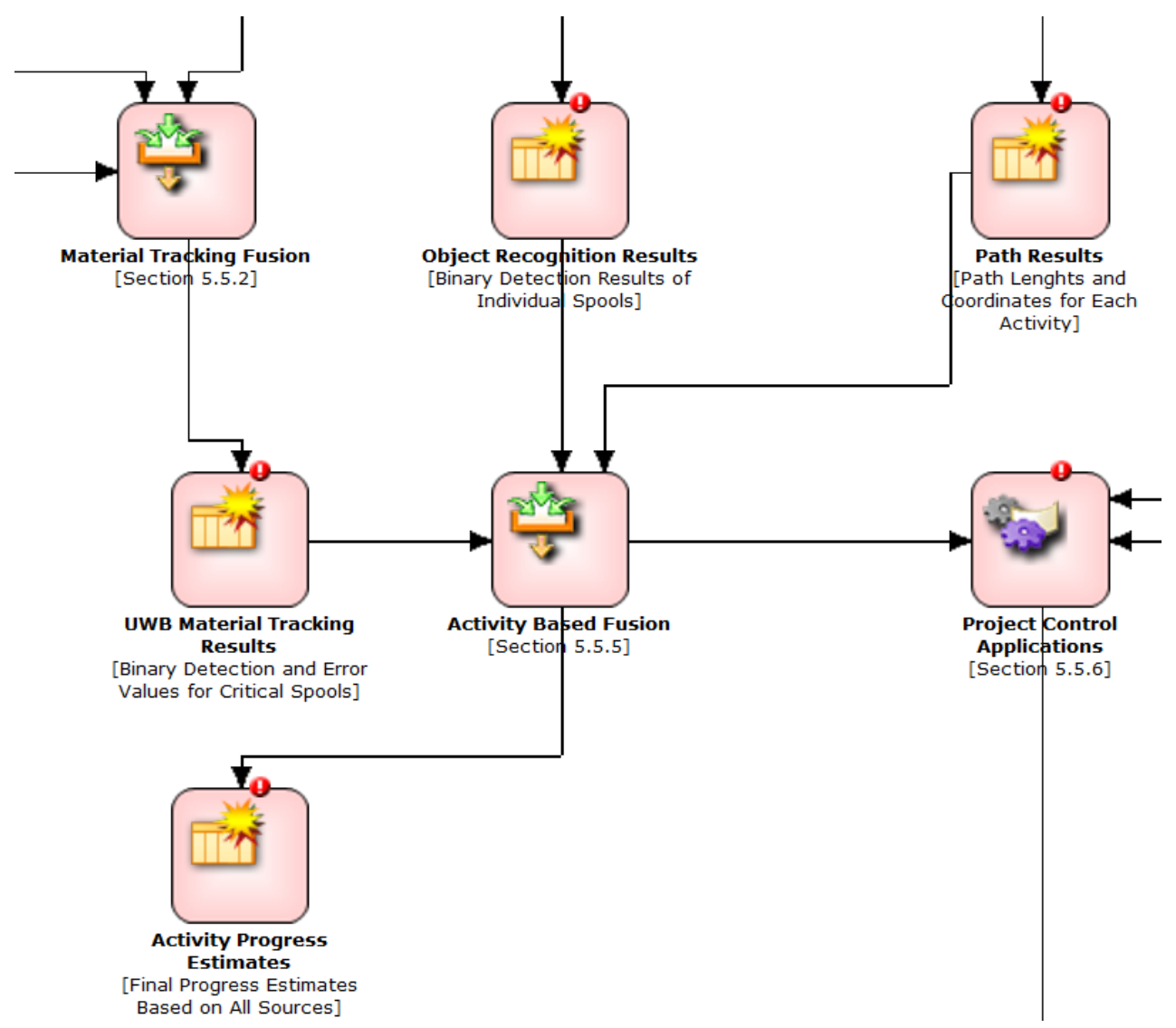

Figure 5-22: Activity-Based Fusion Engine 
Existing material-tracking algorithms and object recognition algorithms that use the 3D model as a-priori information tend to simply compare the as-built location to the as-planned model and therefore do not account for deviations from design criteria, which may lead to significant errors in the estimated progress of the work that has been completed on a project. These deviations from design locations can vary significantly, depending on the type of project.

Figure 5-23 shows the relative amount of design changes or deviations from the original design that may occur during the life of projects. This graph has been constructed by the author based on the input of the industry partners at Aecon Construction and SNC Lavalin. As shown in this figure, concrete structures may have only minimal changes after the start of construction, and there are no changes expected after construction is approximately $20 \%$ completed.

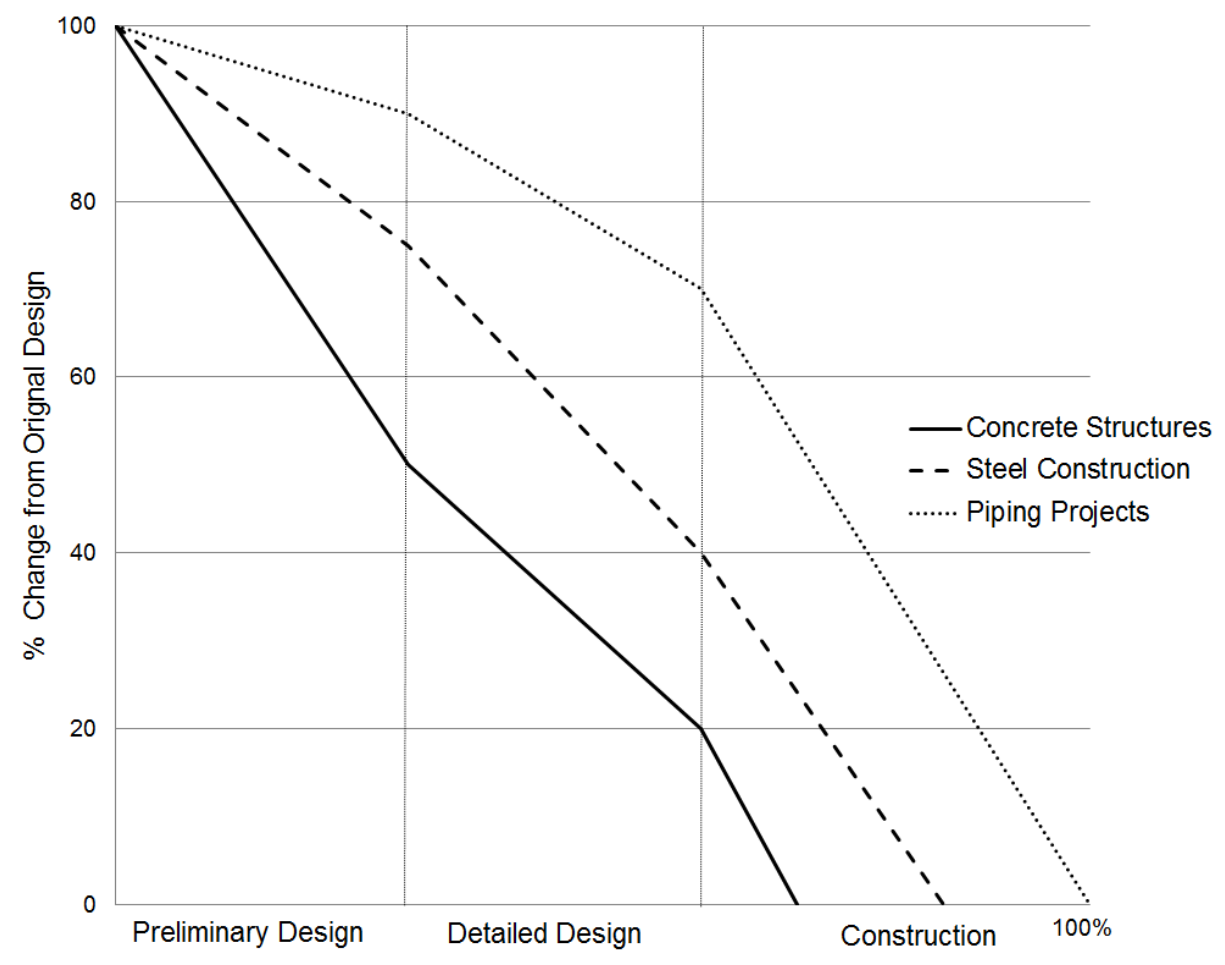

Figure 5-23: Percentage Design Change during the Life of Projects for Categories of Construction Work

Steel structures are more flexible, since the detail changes can be implemented even when the construction has started, but even with steel structures, shortly after the construction progress 
passes the $50 \%$ mark, no design changes are expected. Piping projects, however, may have a very high number of changes from the original design, even during the construction phase. In fact, a number of piping components may have to be field routed, in which case, up to the completion of the construction phase the as-built model may be very different from the asplanned models. In other words, during the construction phase, a concrete column would be subject to significantly fewer or no deviations from the designed location in comparison to a pipeline, which could even be primarily field-routed.

The significance of the difference between these types of construction is that even though automated object recognition and material- or asset-tracking algorithms that use the 3D CAD model as a-priori information may be accurate for concrete or steel structures (assuming that the existing 3D model has been completely updated with design changes and that model is at the site), they will typically be significantly ineffective and inaccurate for piping projects. Due to the differences from the designed locations created by field-routing variations, object-recognition techniques cannot differentiate between a pipeline that has not been installed and one that has been installed with a deviation from its designed location greater than the software threshold, usually a couple of centimeters. However, these two situations present completely different states for a particular pipeline, which has a significant impact on the progress, and consequently, the earned value of the project.

The activity-based fusion engine in the fusion model developed herein is responsible for combining the data from the object-recognition algorithms with the data from other sources of information that were gathered in the field, including UWB material- and activity-tracking data. At this stage in the fusion model, the fusion software evaluates both the progress of the installation activity and the deviations from the as-planned model. This functionality is one of the unique advantages of the developed fusion model compared to traditional material-tracking methods for the purposes of construction progress tracking. The following subsections present the details of the fusion algorithms for the Activity-Based Fusion stage.

\subsubsection{Fuzzy Material-Tracking Operations}

An experiment was conducted to evaluate fuzzy logic as a fusion engine. The results are described in this section. 
Deviations in the installed locations of critical spools from their as-planned locations are initially calculated based on the UWB material-tracking data. These deviations are called "error values." Three functions were created to represent the fuzzy set of error values that indicate an installation at the correct location (ICL), an installation at a wrong/different location (IWL), and the spool delivery status (DEL). These functions were calibrated based on the experimental UWB results, which were also a function of time. The trend line that was used for calibrating these functions is presented in Figure 5-24. This trend line represents the degradation of UWB position accuracy over time due to site congestion for the research project site. The details of the experimental results for the UWB performance evaluation and the deterioration in its performance over the duration of the project can be found in Shahi et al. (2011), a summary of which is presented in Section 4.2 of this thesis.

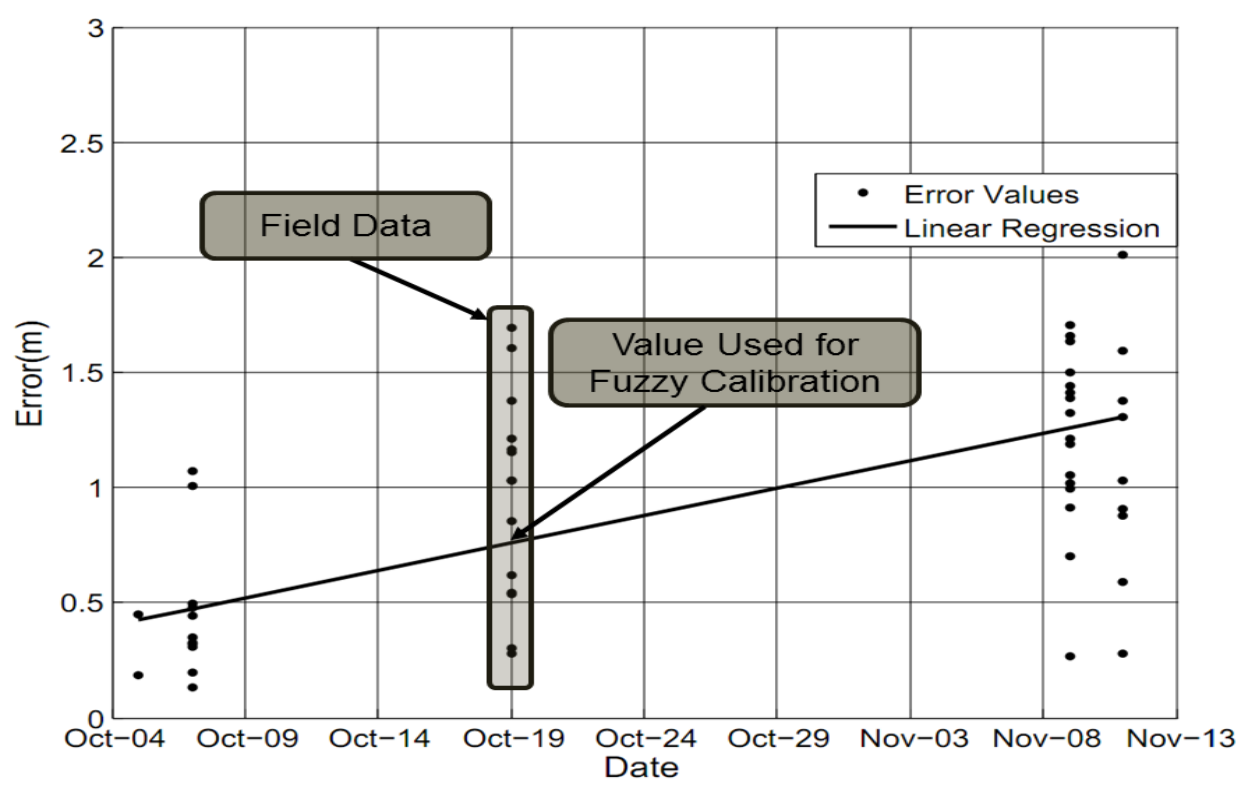

Figure 5-24: Error Trend for Pipe Measurement Points over Time

Sample fuzzy membership functions for the installation activity are shown in Figure 5-25. This figure shows that three states are defined for each critical pipe spool, depending on the distance between its as-built detected location and its as-planned location. This distance is also called its corresponding error value. These fuzzy membership functions are calibrated for each date, based on the UWB performance results shown in Figure 5-24. 
The fuzzy interval between Installed Correct Location (ICL) and Installed Wrong/Different Location (IWL) corresponds to the $95 \%$ confidence interval of the UWB performance data at a specific date. Therefore, if the error values are beyond the upper bound of the $95 \%$ confidence interval for each date but below the next threshold, that particular pipe spool is considered installed, but at a location that differs from its as-planned location. The upper threshold for considering a pipe spool installed at a wrong location refers to the fuzzy region between the Installed at Wrong/Different Location (IWL) and Delivered (DEL) functions. The fuzzy region here is much wider than the previous region because there is a much greater level of uncertainty in the data.

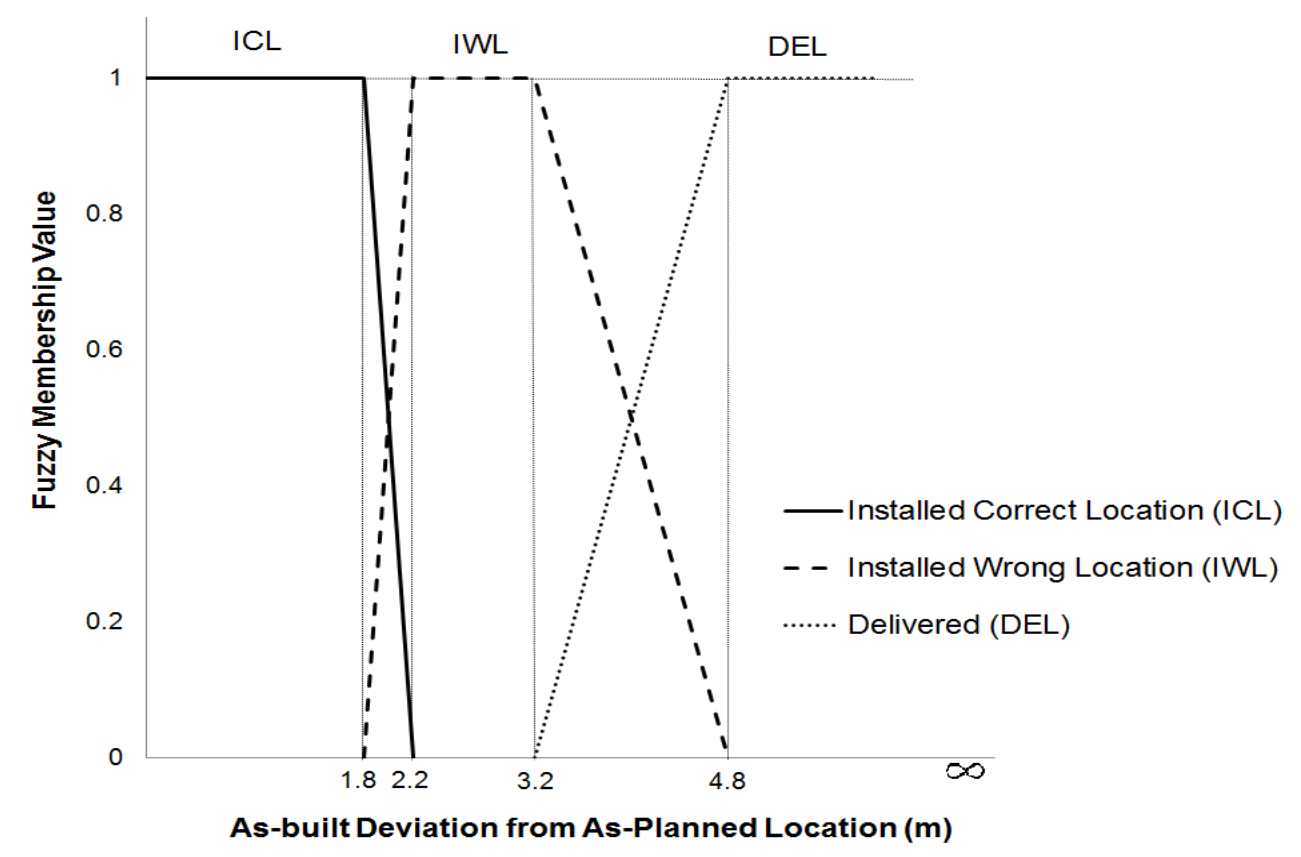

Figure 5-25: Sample Fuzzy Membership Functions

The second fuzzy region was established based on expert opinion, with consideration of the height of the installation of the pipes. For example, once the error reaches the height of the installation as measured from the floor, it would be reasonably safe to assume that the pipe has been delivered, is sitting on the floor, and has not been installed. The third membership function in the installation activity, "Delivered," feeds into the Delivery activity. If a critical spool is not detected at all, no error value is recorded, and the spool is therefore considered "not delivered." The critical spool error values were input within the fuzzy set, and a middle of maximum 
defuzzification process was performed in order to determine the correct installation parameters for the critical spools.

The fuzzy logic engine was originally designed to deal with conflicting and fuzzy input from multiple sources of information. It was tested with a few hypothetical cases to verify its functionality. However, with the development of other fusion engines and considering the quality, variety, and depth of data collected on this implementation site, the impact of the fuzzy logic engine was reduced to a minimum to the point where the fuzzy operations could be simplified to a number of classical logical reasoning algorithms. It is expected that in future implementations of the model, there may be a need for further emphasis on the fuzzy operations. This is recommended as future research.

\subsubsection{Laser Scanning and Material-Tracking Data Fusion}

A schematic representation of a pipeline is presented in Figure 5-26 in order to illustrate the definitions of the terms that are used in the algorithms presented in this section. The dark pipe pieces (indicated by $\mathrm{CR}$ ) are the "critical pipe spools" and therefore would have permanent UWB tags attached to them, while all other spools are considered generic. For example, CR1-2 indicates the second critical spool on pipeline 1. "Intermediary spools" associated with CR1-2 are defined as the generic spools that are halfway between CR1-1 and CR1-2 in addition to those halfway between CR1-2 and CR1-3, as shown in Figure 5-26.

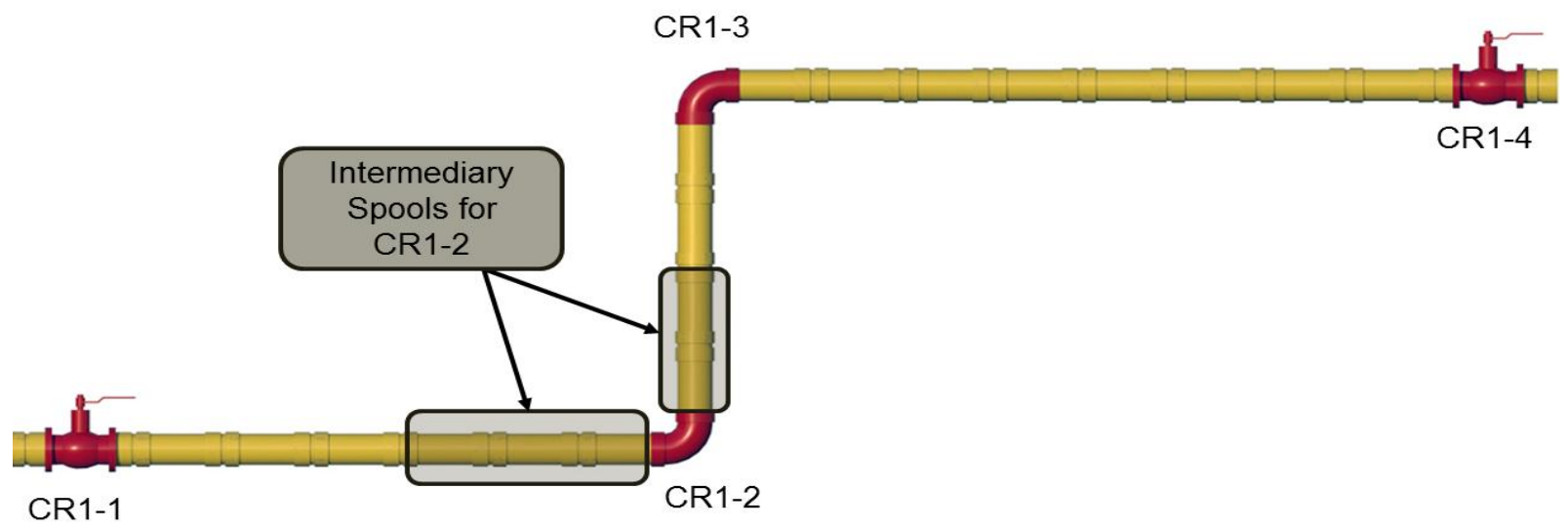

Figure 5-26: Schematic Representation of a Pipeline 
In cases where the number of spools between two critical spools is not an even number, the first critical spool is assigned an extra intermediary spool. Critical spool CR1-2 and its associated intermediary spools are considered the "line segment" of CR1-2.The data collection and fusion algorithms of the model presented in this thesis were verified using a number of simulated pipeline activities, with each line having 2 to 3 critical spools and 8 to 10 generic spools.

The point readings were taken using a UWB positioning system in the field, and a variety of pipeline configurations were then simulated between these points in order to test the software and the fusion algorithms. Delivery, installation, welding, and inspection activities were also simulated using the data collected in the field in order to show the proof of concepts for the fusion algorithms presented in this chapter. Table 5-3 and Table 5-4 summarize the UWB material-tracking results and laser-scanning results generated for two of the sample lines.

Table 5-3: Sample Input Values for Line 1

\begin{tabular}{cccc}
\hline $\begin{array}{c}\text { Line 1 } \\
\text { Spools }\end{array}$ & $\begin{array}{c}\text { UWB Error } \\
(\mathbf{m})\end{array}$ & $\begin{array}{c}\text { Laser } \\
\text { Scanning }\end{array}$ & $\begin{array}{c}\text { Percent } \\
\text { Contribution }\end{array}$ \\
\hline *Spool1_1 & 0.15 & 1 & $10.2 \%$ \\
Spool1_2 & - & 1 & $10.2 \%$ \\
Spool1_3 & - & 1 & $4.5 \%$ \\
Spool1_4 & - & 0 & $4.5 \%$ \\
*Spool1_5 & 0.24 & 0 & $10.9 \%$ \\
Spool1_6 & - & 0 & $10.9 \%$ \\
Spool1_7 & - & 0 & $3.8 \%$ \\
Spool1_8 & - & 0 & $3.8 \%$ \\
Spool1_9 & - & 0 & $20.6 \%$ \\
*Spool1_10 & - & 0 & $20.6 \%$ \\
\hline
\end{tabular}

Table 5-4: Sample Input Values for Line 2

\begin{tabular}{cccc}
\hline $\begin{array}{c}\text { Line 1 } \\
\text { Spools }\end{array}$ & $\begin{array}{c}\text { UWB } \\
\text { Error } \\
(\mathbf{m})\end{array}$ & $\begin{array}{c}\text { Laser } \\
\text { Scanning }\end{array}$ & $\begin{array}{c}\text { Percent } \\
\text { Contribution }\end{array}$ \\
\hline${ }^{*}$ Spool2_1 & 0.21 & 1 & $8.2 \%$ \\
Spool2_2 & - & 1 & $8.2 \%$ \\
Spool2_3 & - & 1 & $16.6 \%$ \\
${ }^{*}$ Spool2_4 & 0.13 & 1 & $16.6 \%$ \\
Spool2_5 & - & 0 & $22.8 \%$ \\
Spool2_6 & - & 0 & $22.8 \%$ \\
Spool2_7 & - & 0 & $2.4 \%$ \\
${ }^{*}$ Spool2_8 & 0.11 & 0 & $2.4 \%$ \\
\hline
\end{tabular}


The spools that are identified with an asterisk are critical spools and are hence the only ones that would have a UWB tag attached. No UWB reading was therefore expected for the other spools. The simulated 3D image object recognition data are listed in the second column, with a value of 1 indicating the recognition of the spool, and 0 representing no recognition. The last column indicates the relative percentage value of the spool in comparison to the entire line. For this research, the percentage contribution was calculated as the length of the spool divided by the total length of the line, but other weighted averages could be used in other applications, if required.

The object recognition algorithms that use the 3D CAD models as a-priori information have reported recognition results with an $85 \%$ to $100 \%$ level of accuracy for building structural components (Bosché, 2010). In this context, accuracy is defined as the percent of the object that were present and were recognized using this methods. However, a very basic assumption in this reported level of accuracy was that all objects were expected to be at the same place as their as-planned location, within very minimal tolerance values. Although this assumption may be valid for most structural elements in a building, such as columns and floors, its rationality drops significantly in the case of piping projects. It is very common for piping lines to be fieldrouted or for their routes to be changed due to congestion at the sites, particularly in the case of industrial projects, where the areas around the installation of piping and duct lines can become extremely congested. For individual piping projects, traditional object-recognition results would therefore fail to provide any reliable means of progress estimation.

Another challenge with piping projects is that as the site becomes congested, the probability of pipes being visible is significantly reduced. For example, during the implementation project, in a number of sections where the duct lines were installed, all of the pipes behind the ductwork were blocked and hence could not be detected by the 3D laser scans. It was not practical to assume that the model would work only if direct line of sight were maintained between all segments of the pipelines and the 3D scanner. This fusion level was designed to make allowances for such blockage of pipelines as well as for the problem of field-routing or installation at locations different from the planned locations.

In this study, a number of fusion algorithms were developed using a "vicinity detection" technique in order to deal with the challenges explained above. The following are a number of 
scenarios for a variety of input combinations that have been fed into this stage by the previous fusion processes, along with the corresponding output of the fusion algorithms at this stage:

- If a critical spool is classified as Installed Correct Location (ICL) by the previous stage of the fusion model (based on UWB positioning data) and more than $10 \%$ of the intermediary spools have been recognized by the automated object-recognition algorithms from the 3D laser scans, then the segment of the pipeline that includes this particular critical spool is classified as Installed Correct Location (ICL).

- If a critical spool is classified as Installed Correct Location (ICL) by the previous stage of the fusion model but less than $10 \%$ of the intermediary spools have been detected by the object-recognition algorithms from the 3D scans, it is concluded that even though this particular spool has been installed close to its as-planned location, the rest of the intermediary spools have been installed in a different location since they have not been detected by the object-recognition algorithms. The line segment containing this particular critical spool is therefore classified as Installed Wrong Location (IWL).

- If a critical spool is classified as Installed Wrong/Different Location (IWL) and more than $50 \%$ of the spools have been recognized by the automated object-recognition algorithms from the 3D scans, it is concluded that the line has been installed in the correct location despite the fact that one critical spool may seem slightly further from its designed location. In this case, the line segment containing that particular critical spool is therefore considered to be Installed Correct Location (ICL).

- If a critical spool is classified as Installed Wrong/Different Location (IWL) and less than $50 \%$ of the spools have been recognized by the automated object-recognition algorithms from the 3D scans, it is concluded that the line segment containing that particular critical spool is Installed in Wrong/Different Location (IWL).

- If a critical pipe spool is classified as Delivered, the line segment containing that particular critical spool is also deemed to be Delivered. It should be noted that, from a supply chain point of view, only the delivery of critical spools is significant because, as 
generic spools, the remainder have minimal to no lead time and are hence non-critical. Therefore, if all critical spools have, in fact, been delivered to the site, the entire delivery activity is deemed to have been completed. This assumption can be revisited in future implementation of the model and other manual or automated sources of information could be integrated to provide a greater resolution to the progress of Delivery activity.

The above logical conditions and relationships address the shortcomings of automated objectrecognition algorithms for cases in which pipelines have been installed at a location different from the as-planned location and in which the pipes have been blocked by other pipes or other objects. A schematic representation of these algorithms is shown in Figure 5-27. The entire code corresponding to this and other levels of fusion is included in Appendix B.

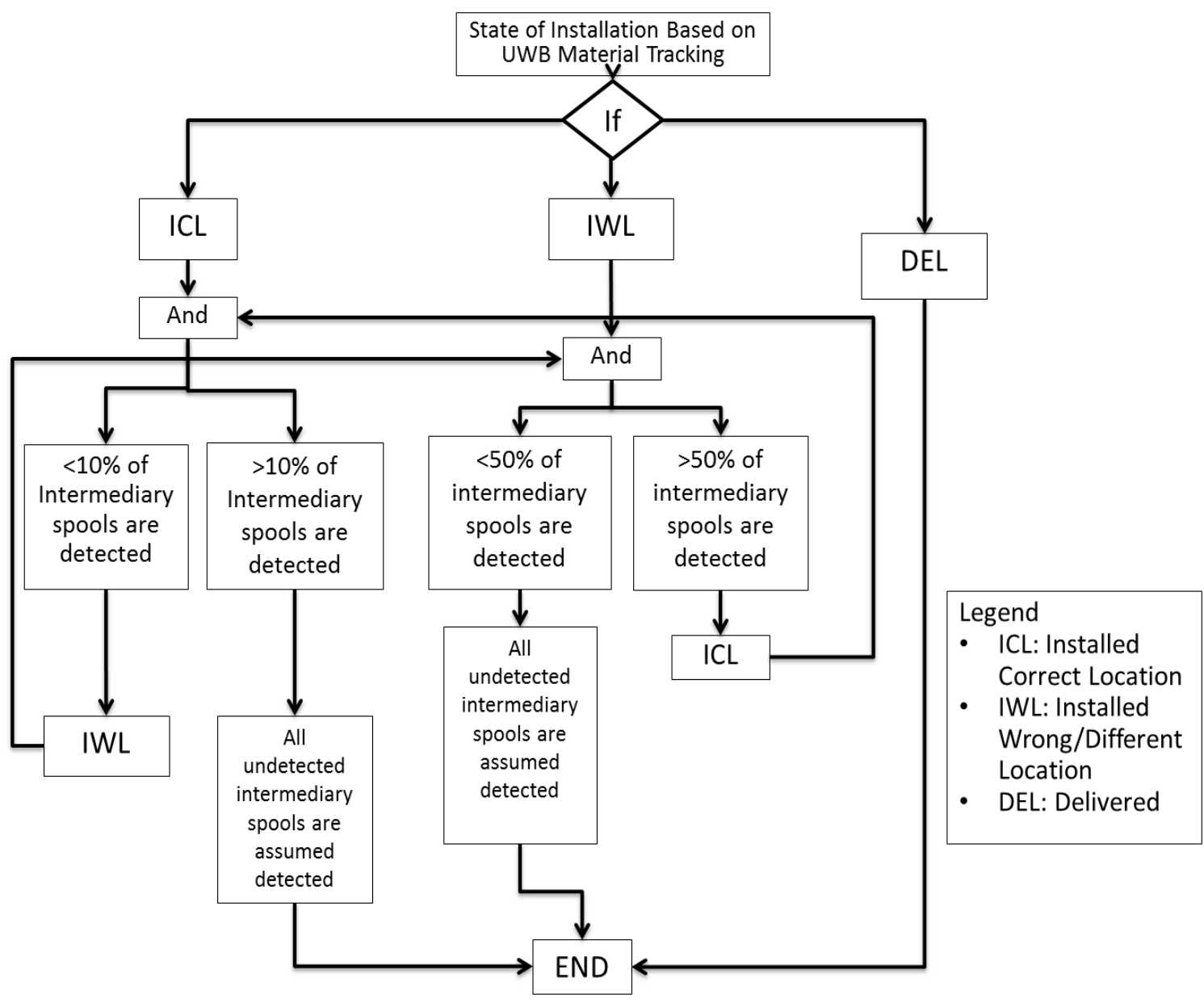

Figure 5-27: Schematic Drawing of Logical Operation Algorithms 
It should be noted that the threshold percentages used in these logical algorithms were assigned through expert opinion and could not be optimized using the data collected for and the path simulations conducted in this research, primarily because the technology for automated object recognition for pipelines had not been fully developed at the time this research was completed. However, it is recommended that the thresholds in these algorithms be optimized using data from a full implementation project.

\subsubsection{Activity-Tracking Fusion}

By this stage in the Activity-Based Fusion algorithms, the UWB material-tracking information and object-recognition results from the 3D scans have been successfully integrated. However, to take advantage of the full capabilities of the fusion model and also to enhance the results even further, the output of the above fusion algorithms is then combined with the activitytracking data. A sample of the paths collected in the field for all four lines, on a given date, is presented in Table 5-5. As indicated in this table, the paths did not necessarily originate at the beginning of the line, but the starting point of the path was recorded at the data collection stage. The activities that do not have a path length associated with them were either not completed or completed but not measured on the date this particular set of data were collected.

Table 5-5: Sample Path Generation Results (from a given date)

\begin{tabular}{cccc}
\hline Line & Activity & Path Length & Start Spool \\
\hline Line 1 & Installation & 11.98 & Spool1_3 \\
& Welding & 8.90 & Spool1_1 \\
& Inspection & 6.97 & Spool1_1 \\
\hline Line 2 & Installation & 12.92 & Spool2_5 \\
& Welding & 11.76 & Spool2_1 \\
& Inspection & - & - \\
\hline Line 3 & Installation & 15.22 & Spool3_5 \\
& Welding & 14.10 & Spool3_1 \\
& Inspection & - & - \\
\hline Line 4 & Installation & 7.14 & Spool4_1 \\
& Welding & - & - \\
& Inspection & - & - \\
\hline
\end{tabular}

The extraction of the activity-tracking data and path-generation algorithms has been discussed in Section 5.5.4 with respect to Path Generation for Indicator Tags. The outcome of that process 
is the list of paths, their corresponding activities, their lengths, and the associated accuracy levels. The start location of each path is also known. Since the average absolute errors in the path readings were less than $6 \%$, the data were deemed to be accurate, and confidence intervals were not considered for the readings. At this fusion stage, the start location and the length of the path are known, and this information is compared against the 3D CAD model for a given pipeline. Thus, for example, for the Installation path on pipeline 1, the total length of the path starting from the first spool is matched with the length of the paths for sequential spools from the same starting point on the CAD model. All spools whose lengths are completely within the measured length are deemed "installed." For the last spool on the path reading, a tolerance of half of the length of the last spool was used, in which case, if at least half of the length for the last spool matches a path that was detected, the spool is considered installed. This algorithm is presented in Figure 5-28.

For intermediary spools that are recognized only through this method, the best description is "Installed at Wrong/Different Location", because if they have been installed at the correct location, they should be at least partially recognized using the 3D scans and would be deemed installed based on the algorithms shown in Figure 5-27.

The data related to inspection and welding is also processed during this stage. It should be noted that, for inspection and welding activities, the only source of data is the generated path data. Two algorithms nearly identical to the one shown in Figure 5-28 were developed as a means of detecting the number of spools welded and inspected. The only variation for the Welding and Inspection activities is that spools can be deemed Welded only if they have previously been detected as Installed by any of the algorithms. If spools are missing an installation status but have been detected as Welded or Inspected, the path is deemed Inaccurate, and for that particular path, a request is sent to the user for either recapture of the path data or manual confirmation.

Table 5-6 and Table 5-7 summarize the activity-based fusion results for the data set that was previously presented on lines 1 and 2, respectively, using the algorithms presented in this section. Perfect performance of the model at this level was expected because the model had been calibrated based on the simulated paths generated from the field experiments, and therefore, for the particular set of data in this project, the results of the model are completely 
accurate. However, as explained in the last chapter of this thesis, once the developed model is fully employed on a construction site, further calibration and adjustments may be necessary.

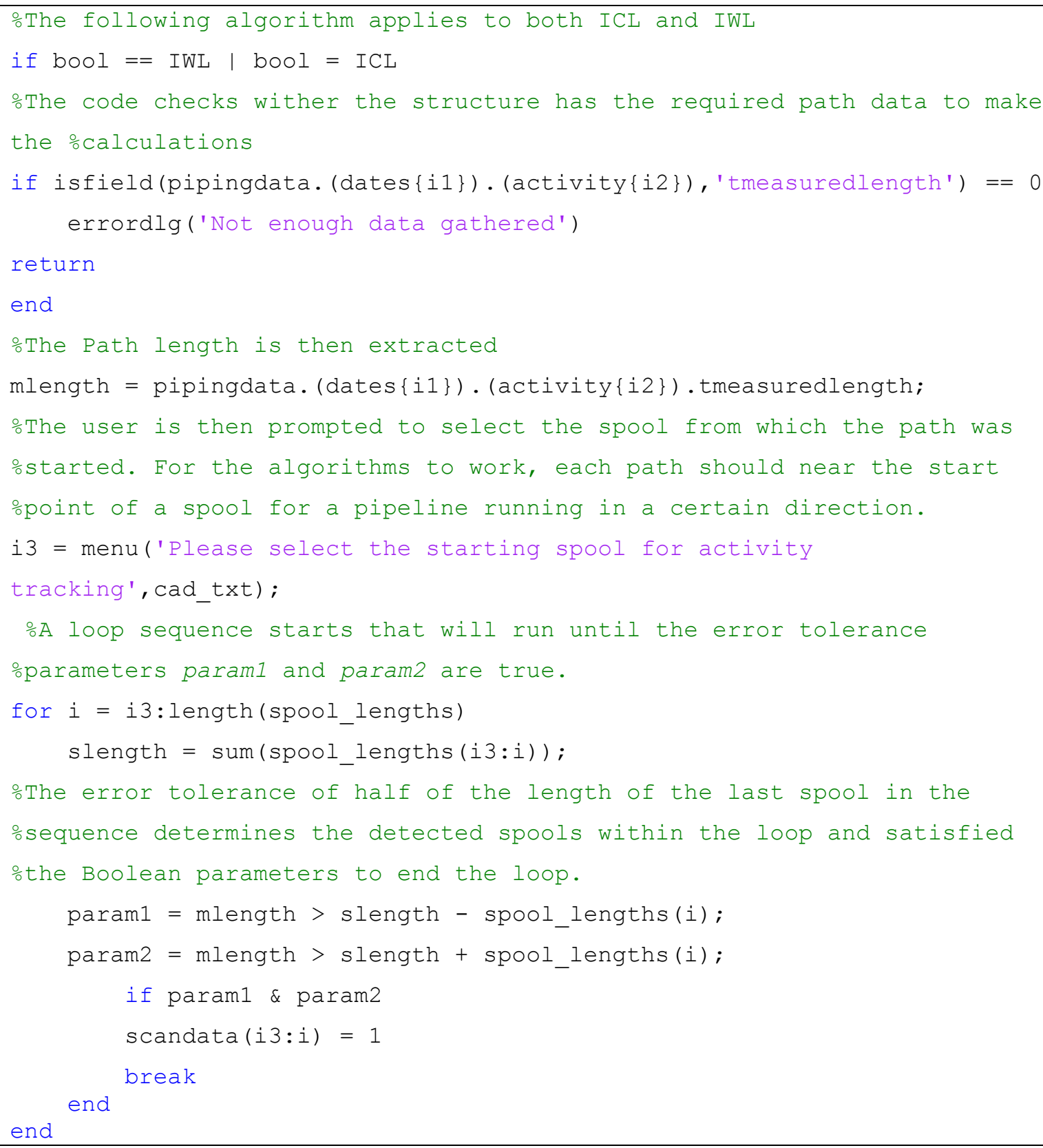

Figure 5-28: Activity Fusion Based on Generated Path Data 
Table 5-6: Sample Activity-Based Fusion Results for Line 1 (for a given date)

\begin{tabular}{ccccccc}
\hline Line 1 & \multicolumn{2}{c}{ Installation Progress } & \multicolumn{2}{c}{ Welding Progress } & \multicolumn{2}{c}{ Inspection Progress } \\
\hline Spool & Actual & Estimated & Actual & Estimated & Actual & Estimated \\
${ }^{*}$ Spool1_1 & 1 & 1 & 1 & 1 & 1 & 1 \\
Spool1_2 & 1 & 1 & 1 & 1 & 1 & 1 \\
Spool1_3 & 1 & 1 & 1 & 1 & 0 & 0 \\
Spool1_4 & 1 & 1 & 0 & 0 & 0 & 0 \\
${ }^{*}$ Spool1_5 & 1 & 1 & 0 & 0 & 0 & 0 \\
Spool1_6 & 1 & 1 & 0 & 0 & 0 & 0 \\
Spool1_7 & 0 & 0 & 0 & 0 & 0 & 0 \\
Spool1_8 & 0 & 0 & 0 & 0 & 0 & 0 \\
Spool1_9 & 0 & 0 & 0 & 0 & 0 & 0 \\
${ }^{*}$ Spool1_10 & 0 & 0 & 0 & 0 & 0 & 0 \\
\hline
\end{tabular}

Table 5-7: Sample Activity-Based Fusion Results for Line 2

\begin{tabular}{ccccccc}
\hline Line 2 & \multicolumn{2}{c}{ Installation Progress } & \multicolumn{2}{c}{ Welding Progress } & \multicolumn{2}{c}{ Inspection Progress } \\
\hline Spool & Actual & Estimated & Actual & Estimated & Actual & Estimated \\
${ }^{*}$ Spool2_1 & 1 & 1 & 1 & 1 & 0 & 0 \\
Spool2_2 & 1 & 1 & 1 & 1 & 0 & 0 \\
Spool2_3 & 1 & 1 & 1 & 1 & 0 & 0 \\
${ }^{*}$ Spool2_4 & 1 & 1 & 1 & 1 & 0 & 0 \\
Spool2_5 & 1 & 1 & 0 & 0 & 0 & 0 \\
Spool2_6 & 1 & 1 & 0 & 0 & 0 & 0 \\
Spool2_7 & 1 & 1 & 0 & 0 & 0 & 0 \\
${ }^{*}$ Spool2_8 & 1 & 1 & 0 & 0 & 0 & 0 \\
\hline
\end{tabular}

At the end of the fusion and logical processes and the operation of the algorithms in the activitybased data fusion model presented in this thesis, a progress estimate is obtained and reported for each individual activity, using the following strategies:

- For the delivery activity, since only the delivery of the critical spools is deemed significant, the number of critical spools detected is divided by the total number of critical spools as defined by the user in order to estimate the progress of the delivery activity.

- For the installation activity, each spool is assigned a Boolean value as a result of the fusion processes explained in this chapter. The corresponding Boolean values for the pipe spools are multiplied by the percentage that each spool contributes to the overall progress of the installation activity and then summed in order to produce the total activity 
progress estimate. In the current state of the software, the percentage contribution of each pipe segment is calculated based on the length of that segment.

- For the welding and inspection activities, the number of joints detected is compared to the total number of joints on each pipeline in order to assess the progress of each activity on any particular pipeline.

Using the above strategies, the progress of each activity is calculated for each line. Using the relative contributions of each activity to the progress of the line, as illustrated in Figure 3-1, the progress of each line is also calculated. Table 5-8 summarizes the activity progress estimations as well as the estimated total progress of each line for four sample lines.

Table 5-8: Sample Activity Progress Results

\begin{tabular}{ccccc}
\hline Activity & Line 1 & Line 2 & Line 3 & Line 4 \\
\hline Delivery & $67 \%$ & $100 \%$ & $100 \%$ & $67 \%$ \\
Installation & $51 \%$ & $100 \%$ & $100 \%$ & $24 \%$ \\
Welding & $30 \%$ & $50 \%$ & $40 \%$ & $0 \%$ \\
Inspection & $20 \%$ & $0 \%$ & $0 \%$ & $0 \%$ \\
\hline Total Progress & $\mathbf{5 1 \%}$ & $\mathbf{8 5 \%}$ & $\mathbf{8 3} \%$ & $\mathbf{3 3} \%$ \\
\hline
\end{tabular}

The activity-based progress estimates are extracted and reported as one of the main outputs of the fusion model presented in this thesis. These activity-based progress estimates are also used as input for the Project Control Applications fusion and analysis engine, which makes this fusion model appropriate for a wide range of other applications. This engine and the applications it enables are presented in the next section.

\subsubsection{Project Control Applications}

Obtaining activity-based progress estimates enables a number of applications that have previously been either not feasible or not efficiently implemented with traditional object-based progress estimates. Two applications were investigated as part of this research in order to demonstrate the powerful advantages of activity-based data fusion and activity-based progress estimates: earned value estimates and the automated updating of project schedules. These two 
applications were built directly into the main graphical user interface (GUI) of the software developed in this research, as shown in Figure 5-29.

This subsection explains the capabilities of the developed model with respect to these two applications. The code that enables these applications is included in Appendix B.

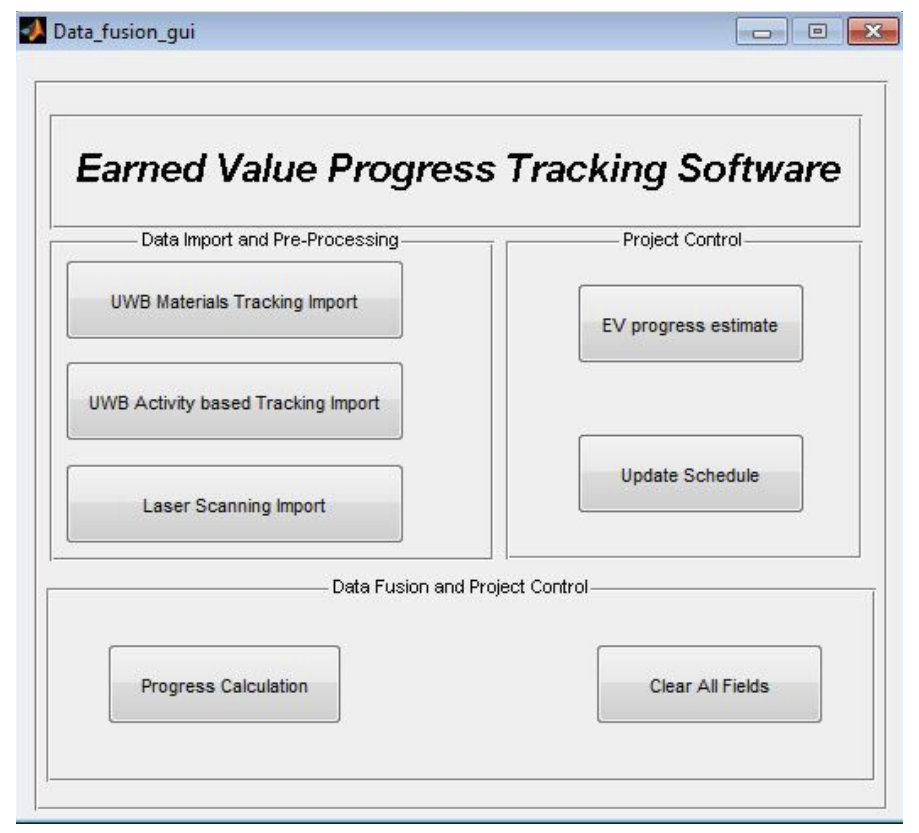

Figure 5-29: Graphical User Interface of the Fusion Model

\subsubsection{Estimate of the Earned Value of the Project}

In a construction project, payment reports generally represent the contractor's estimate of the progress of the project based on the amount billed, as shown in Figure 5-30. No automated means of assessing the accuracy of these reports currently exists, and the claims made by the contractor have to be verified based on visual inspection and the experience of the general contractor's upper management team. However, using activity-based data fusion and progress estimates, the standard payment template can be converted into activities and sub-activities, which can then be tracked individually. The methodology developed in this research has the ability to accommodate all of the activities in the subcontractor's payment form except for the "mobilization" item. 


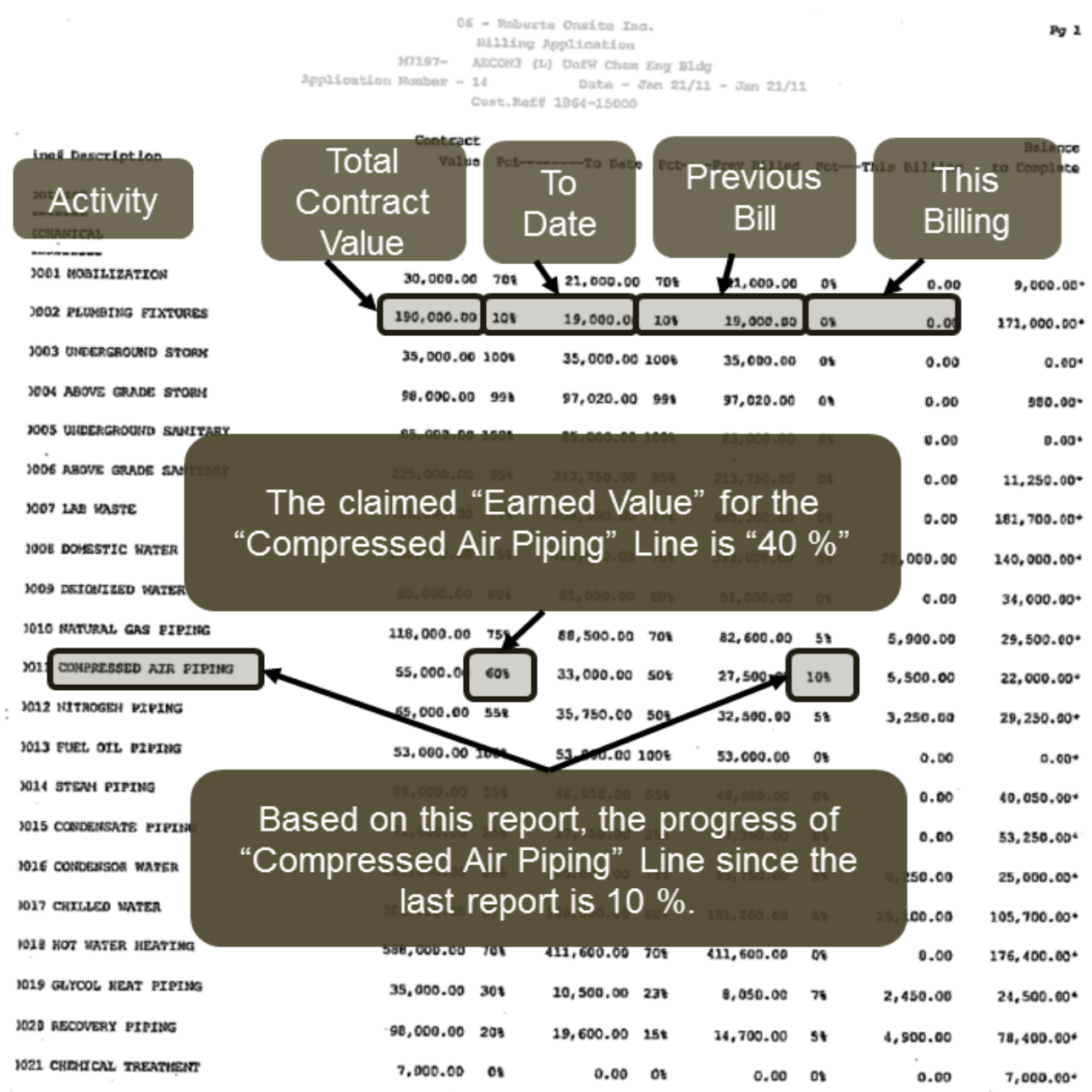

Figure 5-30: Sample Contractor Payment Report

Once the activity progress is obtained using the model presented in this thesis, the percentage progress is multiplied by the total cost budgeted for that particular activity, and an earned value estimate for each activity is obtained. This analysis can be performed for the activity level or for the overall progress of the line. Table 5-9 illustrates an example showing how the earned value report can be generated, using assumed initial contract values. In the sample contractor payment report illustrated in Figure 5-30, which was obtained from Aecon Construction for the Engineering 6 building project at the University of Waterloo, the level of granularity was limited to the lines. To accommodate the existing contract reports, the overall line progress estimates were therefore used in Table 5-9. 
Table 5-9: Sample Estimated Earned Value Results

\begin{tabular}{ccccc}
\hline Line & Description & Contract Value & Progress To Date & Earned Value \\
\hline $\mathbf{1}$ & Natural Gas Piping & $\$ 60,000$ & $51 \%$ & $\$ 30,600$ \\
$\mathbf{2}$ & Nitrogen Gas Piping & $\$ 50,000$ & $85 \%$ & $\$ 42,500$ \\
$\mathbf{3}$ & Fuel Oil Piping & $\$ 35,000$ & $83 \%$ & $\$ 29,050$ \\
$\mathbf{4}$ & Compressed Air Piping & $\$ 40,000$ & $33 \%$ & $\$ 13,200$ \\
\hline
\end{tabular}

The earned value estimation helps resolve payment-related conflicts between subcontractors, contractors, and owners. This level of control and verification of the progress of a project also helps in the early identification of cost overruns and project delays at a construction site. The final application of the model developed for this research is automatic schedule updating, which is presented in the next section.

\subsubsection{Automated Schedule Updating}

Updating a schedule using object-based progress estimates has been attempted in the past but with only limited success due to the inherent limitations of object-oriented progress tracking models. Most schedules are activity driven, and therefore an activity-based progress estimate enables a much more efficient and reliable method of automatically and continually updating a construction project schedule. As part of the Project Control Applications stage, the original schedule is uploaded as input and is updated using the activity-based progress estimates. An updated schedule is then created as one form of the output of this stage in the fusion model. The codes and algorithms for automated schedule updating are provided in Appendix B. Future research could investigate the optimal strategy and most efficient and accurate algorithms for the automatic updating of a schedule. The purpose of this section in the thesis is to demonstrate that activity-based progress estimates provide a more compatible solution for this particular application than do object-based estimates.

For this research, the original schedule was in a Microsoft Project format, which was exported to Microsoft Excel and then imported to the MATLAB engine as input. For greater efficiency in the fusion processes, it is important that the list of activities on the schedule and the list on the contractor payment request forms be identical. Figure 5-31 illustrates an example of an original schedule, extracted from Microsoft Project and imported into Microsoft Excel, which was used as input into the Project Control Applications fusion engine. 


\begin{tabular}{|c|c|c|c|c|}
\hline ID & Name & Duration & Start & Finish \\
\hline 1 & Line 1 & $36 . \mathrm{d}$ & $21 / 09 / 20108: 00$ & $09 / 11 / 201017: 00$ \\
\hline 2 & Installation & $29 . \mathrm{d}$ & $21 / 09 / 20108: 00$ & $29 / 10 / 201017: 00$ \\
\hline 3 & Welding & $30 . \mathrm{d}$ & $29 / 09 / 20108: 00$ & $09 / 11 / 201017: 00$ \\
\hline 4 & Inspection & $5 . \mathrm{d}$ & $29 / 10 / 20108: 00$ & $04 / 11 / 201017: 00$ \\
\hline 5 & Line 2 & $26 . \mathrm{d}$ & $07 / 10 / 20108: 00$ & $11 / 11 / 201017: 00$ \\
\hline 6 & Installation & $19 . \mathrm{d}$ & $08 / 10 / 20108: 00$ & $03 / 11 / 201017: 00$ \\
\hline 7 & Welding & $24 . \mathrm{d}$ & $07 / 10 / 20108: 00$ & $09 / 11 / 201017: 00$ \\
\hline 8 & Inspection & $3 . \mathrm{d}$ & $09 / 11 / 20108: 00$ & $11 / 11 / 201017: 00$ \\
\hline 9 & Line 3 & $33 . \mathrm{d}$ & $29 / 09 / 20108: 00$ & $12 / 11 / 201017: 00$ \\
\hline 10 & Installation & $29 . \mathrm{d}$ & $29 / 09 / 20108: 00$ & $08 / 11 / 201017: 00$ \\
\hline 11 & Welding & $15 . \mathrm{d}$ & $25 / 10 / 20108: 00$ & $12 / 11 / 201017: 00$ \\
\hline 12 & Inspection & $1 . \mathrm{d}$ & $11 / 11 / 20108: 00$ & $11 / 11 / 201017: 00$ \\
\hline 13 & Line 4 & $23 . \mathrm{d}$ & $20 / 10 / 20108: 00$ & $19 / 11 / 201017: 00$ \\
\hline 14 & Installation & $23 . \mathrm{d}$ & $20 / 10 / 20108: 00$ & $19 / 11 / 201017: 00$ \\
\hline 15 & Welding & $7 . \mathrm{d}$ & $01 / 11 / 20108: 00$ & $09 / 11 / 201017: 00$ \\
\hline 16 & Inspection & $3 . \mathrm{d}$ & $03 / 11 / 20108: 00$ & $05 / 11 / 201017: 00$ \\
\hline
\end{tabular}

Figure 5-31: Sample Original Schedule Export Used for Input

If the progress is below or above the expected level for a specific date, the due date for the activity is adjusted by using the percentage completed as of that day in order to calculate the time remaining based on pre-established productivity values (linear $\mathrm{ft}$ of piping/day or welds/day). Table 5-10 summarizes the adjustments that were made to the original schedule based on the estimated progress of each activity.

Table 5-10: Schedule Adjustment Based on Estimated Progress

\begin{tabular}{cccccc}
\hline & Activity & $\begin{array}{c}\text { Days } \\
\text { Elapsed }\end{array}$ & $\begin{array}{c}\text { Estimated } \\
\text { Progress }\end{array}$ & $\begin{array}{c}\text { Days } \\
\text { Remaining }\end{array}$ & $\begin{array}{c}\text { New } \\
\text { Duration }\end{array}$ \\
\hline Line 1 & Installation & 29 & $51.20 \%$ & 15 & 44 \\
& Welding & 23 & $30.00 \%$ & 21 & 44 \\
& Inspection & 1 & $20.00 \%$ & 4 & 5 \\
\hline Line 2 & Installation & 16 & $100.00 \%$ & 0 & 16 \\
& Welding & 17 & $50.00 \%$ & 12 & 29 \\
& Inspection & - & $0.00 \%$ & - & - \\
\hline Line 3 & Installation & 23 & $100.00 \%$ & 0 & 23 \\
& Welding & 5 & $40.00 \%$ & 9 & 14 \\
& Inspection & - & $0.00 \%$ & - & - \\
\hline \multirow{2}{*}{ Line 4 } & Installation & 8 & $24.06 \%$ & 18 & 26 \\
& Welding & - & $0.00 \%$ & - & - \\
& Inspection & - & $0.00 \%$ & - & - \\
\hline
\end{tabular}


In the Project Control Applications engine, the original productivity values are used for forecasting purposes, but other strategies could be employed, including a user-defined productivity value for the forecasting calculations. The output of this engine in the form of the updated schedule based on the estimated progress is presented in Figure 5-32. This format is consistent with the format of the file extracted from Microsoft Project and can therefore be easily imported back to that software. Microsoft Project can then be used to recalculate the start and end dates, floats, and critical path based on the original precedence relationships.

\begin{tabular}{|c|c|c|c|c|}
\hline ID & Name & Duration & Start & Finish \\
\hline 1 & Line 1 & $50 . \mathrm{d}$ & $21 / 09 / 20108: 00$ & $29 / 11 / 201017: 00$ \\
\hline 2 & Installation & $44 . \mathrm{d}$ & $21 / 09 / 20108: 00$ & $19 / 11 / 201017: 00$ \\
\hline 3 & Welding & $44 . \mathrm{d}$ & $29 / 09 / 20108: 00$ & $29 / 11 / 201017: 00$ \\
\hline 4 & Inspection & $5 . \mathrm{d}$ & $29 / 10 / 20108: 00$ & $04 / 11 / 201017: 00$ \\
\hline 5 & Line 2 & $29 . \mathrm{d}$ & $07 / 10 / 20108: 00$ & $16 / 11 / 201017: 00$ \\
\hline 6 & Installation & $16 . \mathrm{d}$ & $08 / 10 / 20108: 00$ & $29 / 10 / 201017: 00$ \\
\hline 7 & Welding & $29 . \mathrm{d}$ & $07 / 10 / 20108: 00$ & $16 / 11 / 201017: 00$ \\
\hline 8 & Inspection & $3 . \mathrm{d}$ & $09 / 11 / 20108: 00$ & $11 / 11 / 201017: 00$ \\
\hline 9 & Line 3 & $32 . \mathrm{d}$ & $29 / 09 / 20108: 00$ & $11 / 11 / 201017: 00$ \\
\hline 10 & Installation & $23 . \mathrm{d}$ & $29 / 09 / 20108: 00$ & $29 / 10 / 201017: 00$ \\
\hline 11 & Welding & $14 . \mathrm{d}$ & $25 / 10 / 20108: 00$ & $11 / 11 / 201017: 00$ \\
\hline 12 & Inspection & $1 . \mathrm{d}$ & $11 / 11 / 20108: 00$ & $11 / 11 / 201017: 00$ \\
\hline 13 & Line 4 & $26 . \mathrm{d}$ & $20 / 10 / 20108: 00$ & $24 / 11 / 201017: 00$ \\
\hline 14 & Installation & $26 . \mathrm{d}$ & $20 / 10 / 20108: 00$ & $24 / 11 / 201017: 00$ \\
\hline 15 & Welding & $7 . \mathrm{d}$ & $01 / 11 / 20108: 00$ & $09 / 11 / 201017: 00$ \\
\hline 16 & Inspection & $3 . \mathrm{d}$ & $03 / 11 / 20108: 00$ & $05 / 11 / 201017: 00$ \\
\hline
\end{tabular}

Figure 5-32: Sample Updated Schedule as Output

There are many critical views to CPM scheduling, particularly with respect to activity relationships, and a number of researchers have proposed alternative scheduling techniques, including Critical Path Segments Scheduling (Hegazy \& Menesi, 2010). Investigating the various scheduling techniques was beyond the scope of the current research and therefore CPM scheduling was used simply to demonstrate the capability of the results obtained using the fusion model of this research for the automated updating of construction schedules.

\subsection{Performance Evaluation of the Model}

The activity-based data fusion engine presented in this thesis is the first published research for establishing an activity-based progress estimate for construction sites using a UWB tag 
indicator approach for tracking construction activities. The fusion engine and its components were developed in parallel with the laboratory and field experimental programs of this research because some of the experimental results were needed in order to analyze and model the performance of the input parameters for the model. The fusion engine also included consideration of the automated object recognition results from 3D laser scanning of piping projects, an extension to the object-recognition technology which was being developed simultaneously at the University of Waterloo with the research presented in this thesis. Based on these limitations and circumstances, a full implementation and validation of the engine using empirical data from the field experiments was not feasible and is recommended for future research. However, the engine was validated using simulations based on the experimental data collected in the field, as explained in a number of previous sections of this chapter. In this section, the functionalities of the engine and the broader model within which it was implemented are compared with current practices on construction projects as well as with other automated construction progress tracking research work, following which, the advantages of the developed model and its contributions to the body of knowledge are presented.

\subsubsection{Existing Progress Tracking Methods for Construction Sites}

Aecon Construction, one of Canada's largest and most diverse construction and infrastructure development companies, was the general contractor for the Engineering 6 building at the University of Waterloo where the majority of the data for this research was collected. Primarily because of the absence of an objective and systematic method of tracking the progress of the construction activities at this site, Aecon's top management team was very interested in the objectives of this study related to providing a mechanism for automated construction progress tracking. In fact, at the Engineering 6 project, the only systematic and documented progresstracking method for the piping activities was the payment request forms submitted by the subcontractors to the general contractor.

The complete set of payment request forms issued by the piping subcontractor to Aecon were provided for this research, an example of which is shown in Figure 5-30. The blurry quality of parts of this figure is due to the requirement to obscure confidential information regarding the subcontractor of the project. For the same reason, the actual payment request forms are not included in the appendices of this thesis. As shown in this figure, for each activity or contract 
item such as "compressed air piping," the subcontractor reports the total value of the item per the contract, the amount that is billed to date, the amount billed on the last payment form, and the resulting amount being billed in the given period. The last item is claimed to be the progress for that particular activity in the last 30 days, that is, the progress that has occurred since the last bill payment. Interviews with the subcontractor revealed that the payment reports are "best estimates" based on what their foremen believe to have been completed in the given period. As well, the general contractor usually has no system for checking the claims made by the subcontractor, which in many cases, leads to lengthy and heated discussions between the subcontractor and the general contractor in order to determine the "earned value" of the work that has been completed.

General contractors must deal with numerous strategies that subcontractors may use in their billing requests in order to improve their cash flow, such as over-claiming and banking work. Having an accurate estimate of the progress of the work and the earned value of the work would therefore help the management team of the project in many ways, including dealing with payment requests from subcontractors.

As shown in previous sections of this chapter, the output of the activity-based progress tracking model presented in this thesis is very similar in format to the claims presented by subcontractors and therefore provides a tool that is very easy for project managers to use. In terms of granularity, format, and level of detail provided, the output of the developed fusion model is therefore consistent with industry needs. The next section provides a set of function-based arguments that attest to the advantages of the developed fusion model over existing fusion models for the particular application of construction progress tracking.

\subsubsection{Existing Automated Progress Tracking Models}

Existing data collection models for the particular application of automated progress tracking in construction projects and the corresponding data fusion models have focussed on the automated recognition of objects (Bosché, 2010; Golparvar-Fard, Pea-Mora et al., 2009; C. Kim et al., 2011; Kwon, Bosche, Kim, Haas, \& Liapi, 2004; Teizer, Haas, Caldas, \& Bosche, 2006; Teizer et al., 2007) and on the automated tracking of objects ( Razavi et al., 2009; Razavi \& Haas, 2010). In this research, these models are referred to as object-based fusion models. 
These object-based models have shown promising results for projects in which progress is tracked in terms of a bulk quantity of materials or objects. For example, steel-framed building construction provides a good example for the validation of these object-based models, because the progress of the building project is reported in tons of steel installed, and recognizing the number of objects that have been installed provides an adequate level of detail for that type of progress tracking.

Despite the success of the existing models in providing a relatively accurate estimate of the progress of specific construction projects, many activities involved in a construction project cannot be tracked using these existing object-based models. While piping activities, for example, can account for up to $50 \%$ of the work in industrial building projects, none of the current progress-tracking models can effectively incorporate these activities for two main reasons:

1) Piping activities, as well as many other activities on construction projects, entail specific elements (welding, inspection, etc.) that are not associated directly with the movement or addition of a physical entity at the site and therefore cannot be tracked effectively using object-based models.

2) Most existing object recognition models use the $3 D$ as-planned model of the site as a-priori information for their models. If an object in the as-built point clouds is not found within a small threshold $(2 \mathrm{~cm}-5 \mathrm{~cm})$ of their designed location, then the object is not recognized. As shown in Figure 5-23, the as-planned model may be very different from the as-built model, particularly in the case of piping projects. Object recognition models can therefore not be used effectively even to track the presence of pipes and spools in cases in which field-routing is preformed or in which the installation location is slightly different from the planned location.

The goal of developing the fusion model presented in this thesis was not to replace or compete with existing models. Instead, the objective of the developed fusion algorithms and the activitybased data collection and fusion mechanism was to address the two major shortcomings of the existing models, as explained above. The Path Generation for Indicator Tag engine (presented in section 5.5.4) was designed to address the first problem with respect to tracking activities, 
and the Activity-Based Fusion (presented in section 5.5.5) was developed to address the second shortcoming related to the differences between the as-built models and the as-planned models. The developed model and its engines were calibrated and validated using empirical data where possible and simulations in other cases, as described in the corresponding sections of this thesis.

The structure of the fusion model, as presented in Figure 5-14, was designed so that the accuracy and quality of the progress estimate would be equal to or better than any individual source of information. This goal was achieved by ensuring that the dimensions of the data and the level of granularity were not reduced by the operation of any of the fusion engines in the model. Instead, the numerous sources of information, including the activity-based-tracking, material-tracking, and object recognition results, were used as complementary information, which increased the accuracy and the redundancy of the information in the model. Therefore, from a functional perspective, the results from the developed model would be superior to those obtained with a progress-tracking method that uses any of the individual sources of data alone, but a comprehensive comparison of the performance of the model with that of existing models requires a full implementation on a construction project. As the result of the research presented in this thesis, the developed model is ready for such a full validation and implementation on a construction project from procurement to construction, which is recommended for future research. Chapter 6 presents a data management and sharing system that could be used to manage the numerous data sources for the future field implementation of the model.

\subsection{Summary}

This chapter has described the design process, field experiments, and simulations for the developed activity-based data fusion model which incorporates both structural and nonstructural activities. State-of-the-art material- and event-tracking technologies have been explored, including ultra-wideband (UWB) positioning systems and 3D laser scanning. The model presented in this chapter also demonstrates the ability to fuse data from non-sensory sources of information, such as the Building Information Model (BIM), schedule information, and payment and progress reports. Also presented are the workflows that have been developed for the data fusion mechanisms that enables the model to be applied for automated construction progress tracking. The fusion engines, algorithms, and logical relationships that were 
incorporated have been presented, and for each fusion stage, an explanation has been provided of the input included, the processing algorithms, and the output that was generated.

The design and development of the model were performed in parallel with the field experiments. While future technological advances are required prior to the full implementation of this model, particularly with respect to automated object recognition for pipe lines, the data collected from the field experiments conducted for this research were sufficient for the validation of parts of the model. With the use of the fusion engines that are built into the fusion model, the resulting progress estimate will outperform all existing manual and automated progress estimation systems. The main advantages of the progress estimates obtained from the model developed in this research are the data redundancy that results from the variety of modes and types of data collection as well as the utilization of the activity-based fusion algorithms, which are superior to object-based algorithms with respect to applicability, reliability, and efficiency. In comparison with existing models that provide object-based progress estimates, the activity-based progress estimates obtained using the model presented in this thesis are much more effective and easier to use for a variety of construction management and control applications, such as earned value estimation and automated schedule updating, as described in this chapter. 


\section{Data Management System}

Many applications in the construction industry, including automated construction schedule updating, automated progress tracking, and earned value tracking, require prompt and accurate retrieval of data from a construction site (Kiziltas \& Akinci, 2005). The final objective of this research was to establish a reliable, efficient, and scalable data management system for sharing construction research data, from which a variety of data fusion models could extract the data required. The fusion model presented in this research relied on information that was obtained from numerous sources and was gathered by a variety of parties. To enable successful future implementations of this model, it was important that a system be defined for sharing the data with the various stakeholders involved in a project. Figure 6-1 shows the data management system component of this research with respect to the other aspects of the fusion model as developed during the previous chapters of this thesis.

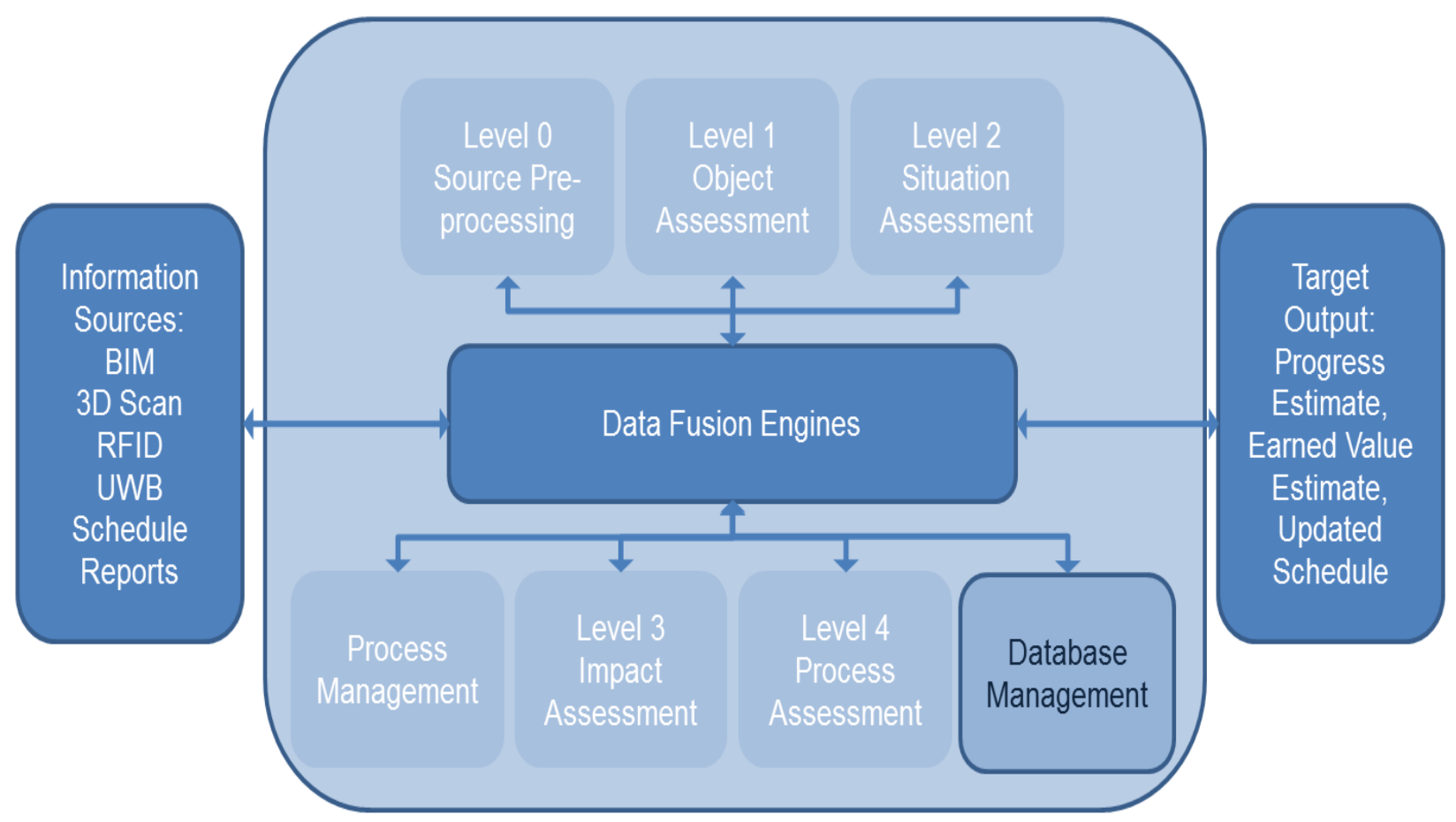

Figure 6-1: Data Management within the Developed Fusion Model 
The current research also had the goal of establishing a benchmark for future data fusion models in the activity-based domain. The enormous amount of data that were collected for the current research project can provide the basis for future research projects in the construction progress estimation domain as well as in many other related domains. The multi-dimensional nature, level of detail, continuity, and comprehensive aspects of the data collected on the site make the information suitable for investigating many aspects of construction related research areas, but only if an effective research data management system could be defined.

In addition to these incentives for developing an effective research data management system, and as explained in the literature review, research funding agencies, such as the National Institutes of Health (NIH), the National Science Foundation (NSF), and the National Institute of Justice (NIJ), have also recently begun to require that all research data produced through their funding be shared with other researchers. A need has therefore arisen for an effective and efficient means of managing and sharing research data.

\subsection{New Electronic Product and Process Management System (EPPMS)}

A review of existing data management models, including web-based cloud and web-based SharePoint models, revealed that no effective system for sharing the raw data obtained from construction research endeavours currently exists. This chapter presents a new electronic product and process management system (EPPMS) that can provide a construction research data management and sharing solution.

\subsubsection{Advantages of Electronic Product and Process Management Systems}

The EPPMS developed through this research is a web-based system that utilizes workflows for automating the collection, management, and dissemination of construction research data. A comparative analysis of the proposed system and the existing web-based cloud and web-based SharePoint systems was conducted with respect to their reliability, content quality control, accessibility controls, collaboration capabilities, and technical simplicity. The results are presented in this section. The developed model was validated using the data collected for the research presented in this thesis. 


\subsubsection{EPPMS as an Integration Tool}

In the construction industry, EPPMSs are used to facilitate the execution of capital projects. An EPPMS links project stakeholders via the internet and system servers. While related systems include building information modeling (BIM), integrated construction project management systems, enterprise resource planning (ERP) systems, and generic electronic document management systems (EDMS), an EPPMS is unique. It differs from a generic EDMS because it directly expresses the project delivery mechanism and processes. An EPPMS does not functionalize business processes such as accounts receivable and payroll, nor does it manage large databases directly, but instead interfaces with business systems such as ERPs, with engineering systems such as CAD, and with project management systems for schedule and cost control, essentially acting as a meta-manager of these systems. At the metalevel, EPPM systems can establish and map information flow, implement project processes, and define contractual and informal relationships in terms of RACI (responsible, accountable, consulted, and informed).

The EPPMS developed through this research for the purposes of data management and data sharing of construction research data differs from previous information management systems because it manages not only information but also knowledge. The nature of an EPPMS as a repository of process-based information makes it an ideal medium for storing information about research processes, resources required, and the dependencies that affect the outcome of a research process. However, the usefulness of an EPPMS extends beyond the capability of acting as a historical repository because it can actually be utilized as an enabler of processes (including fusion) in real-time. In the following section, this functionality is explained in the context of workflow-driven processes.

\subsubsection{Workflow-Driven Structure}

The EPPMS developed in this research employs workflows to further increase automation. A workflow management system, as defined by the Workflow Management Coalition (WfMC), is "a system that completely defines, manages and executes 'workflows' through the execution of software whose order of execution is driven by a computer representation of the workflow logic." Workflows automate the formatting, version updating, dissemination, and verification of 
contributions, making the EPPMS more than just a data filing system. This increased level of automation would be beneficial for any construction research or fusion data management system because it would lessen the resource requirements for data collection, assembly, and dissemination.

The process-based and workflow-driven structure of the EPPMS solution presented in this thesis lends itself very easily to use with data fusion processes. The workflows included in the data fusion model described in Chapter 5 can be built directly into the process-based EPPMS presented in this chapter. Using this strategy, the entire fusion model would function as one of the processes within the main EPPMS for a project, providing accurate, reliable, and frequent progress estimates to all parties involved in the project.

\subsubsection{Flexibility and Collaboration Capabilities}

The EPPMS can be used to handle, edit, track, and view all forms of project documents: text, drawings, images, etc. This feature simplifies the use of the information in the system, thereby further reducing resource requirements for users. The ability of an EPPMS to allow users to view any document regardless of that user's own software provides contributors with formatting freedom. This advantage promotes collaboration within the system because it both increases the technical simplicity of the EPPMS and reduces the resources required of the contributor.

EPPMSs are also highly customizable. Features such as access management, quality/version control, and contributor confidentiality are determined based on the needs presented. For the developed construction research data management system, access would be as open as possible while maintaining the highest quality standards. Ensuring the high quality and integrity of the data is crucial for data fusion processes explained in the previous chapters of this thesis. Membership is limited to construction research institutions in order to ensure the relevance and quality of any contributions being made to the system, which is also checked through the use of quality verification workflows. These workflows automatically send out data contributions to other members for review and validation. As with the web-based SharePoint system, the EPPMS also employs algorithms to compare new contributions with previous versions in an attempt to identify data entry errors 
The EPPMS differs greatly from web-based cloud and SharePoint systems by offering formalized and structured processes for facilitating the recognition of contributors through contributor identification. The content of the system is tied to its contributor so that data sources can and must be referenced if used, and this referencing system is automated through workflows.

The EPPMS can also be a vehicle for system members to communicate with one another, providing a platform for collaboration that, unlike web-based SharePoint systems, includes a social aspect. Members can take advantage of the opportunity to network within their research community and even to form joint research ventures with other scientists and institutions. The profoundly collaborative environment that is offered by the EPPMS is valuable not only to researchers but also to the construction industry at large because it allows the construction research network to act as a whole to further the progress of construction research.

\subsubsection{Comparative Analysis of Data-Sharing Systems}

The EPPMS provides a holistic solution to construction research data management and would be a better construction research data management system than a web-based cloud or a webbased SharePoint system. It is highly automated, simple to use, and customizable. The possibilities that EPPMS present for a collaborative research environment are tremendous. Section 2.9.3 provides a background review on the existing models and a comparison of the three data management systems is summarized in Table 6.1.

\subsubsection{Coreworx Solution as the EPPMS Provider}

Coreworx Inc is a leading example of an EPPMS solution provider in the construction management industry. Coreworx is an innovator in EPPM systems and has achieved a global presence, providing project execution solutions for industry-leading organizations such as Babcock \& Wilcox, Ontario Power Generation, Fluor, Chevron, BHP Billiton, J. Ray, and Husky. Existing Coreworx solutions facilitate information sharing between participants in complex projects that include construction projects, serving a portfolio of more than 500 projects valued at over $\$ 500$ billion across more than 50 countries and with nearly 70,000 users. An EPPMS similar to the one Coreworx offers for mega construction projects has been developed for a construction research data management mechanism. 
Table 6.1 - Comparison of Data Management Systems

\begin{tabular}{|c|c|c|c|}
\hline System & $\begin{array}{l}\text { Wiki-Based Cloud } \\
\text { (Example: Wikipedia) }\end{array}$ & $\begin{array}{l}\text { Web-Based SharePoint } \\
\text { (Example: CII's } \\
\text { Benchmarking and Metrics } \\
\text { Program) }\end{array}$ & $\begin{array}{l}\text { Developed Electronic } \\
\text { Product and Process } \\
\text { Management System }\end{array}$ \\
\hline $\begin{array}{l}\text { Promotion of } \\
\text { Collaboration }\end{array}$ & $\begin{array}{l}\text { Promotes collaboration } \\
\text { though open access. } \\
\text { Collaboration is } \\
\text { critical for ensuring } \\
\text { the accuracy of the } \\
\text { information in the } \\
\text { cloud. Collaboration } \\
\text { in an open } \\
\text { environment leads to a } \\
\text { substantial volume of } \\
\text { information. }\end{array}$ & $\begin{array}{l}\text { Collaborative environment } \\
\text { established through } \\
\text { member participation } \\
\text { requirements. } \\
\text { Diversity of contributors is } \\
\text { somewhat limited through } \\
\text { membership requirements. }\end{array}$ & $\begin{array}{l}\text { Promotes collaboration } \\
\text { through communication } \\
\text { capabilities and member } \\
\text { contributions to overall } \\
\text { efforts. } \\
\text { Formatting freedom } \\
\text { facilitates contribution to the } \\
\text { system. }\end{array}$ \\
\hline Accessibility & $\begin{array}{l}\text { Accessible to anyone, } \\
\text { at any time, via the } \\
\text { internet at no cost. } \\
\text { Information is } \\
\text { provided immediately. }\end{array}$ & $\begin{array}{l}\text { Accessible to paying } \\
\text { members only via the } \\
\text { internet. } \\
\text { Continuous data collection } \\
\text { members can submit } \\
\text { information at any time. }\end{array}$ & $\begin{array}{l}\text { Accessible via the internet. } \\
\text { Access management is } \\
\text { customizable through } \\
\text { membership that is proposed } \\
\text { to be limited to construction } \\
\text { research institutions. }\end{array}$ \\
\hline $\begin{array}{l}\text { Content } \\
\text { Quality }\end{array}$ & $\begin{array}{l}\text { Quality control } \\
\text { problems as a result of } \\
\text { open access to all } \\
\text { content and ever- } \\
\text { evolving articles. } \\
\text { No quality control or } \\
\text { formal review } \\
\text { mechanisms in place. }\end{array}$ & $\begin{array}{l}\text { High-quality standards } \\
\text { critical to SharePoint } \\
\text { success. } \\
\text { Quality control } \\
\text { mechanisms in place, such } \\
\text { as data entry algorithms } \\
\text { and contributor control. }\end{array}$ & $\begin{array}{l}\text { Membership requirements } \\
\text { are one quality control. } \\
\text { Workflows and algorithms } \\
\text { are used as review and } \\
\text { validation mechanisms. }\end{array}$ \\
\hline $\begin{array}{l}\text { Contributor } \\
\text { Compensation }\end{array}$ & $\begin{array}{l}\text { No compensation for } \\
\text { effort or time spent } \\
\text { sharing information. } \\
\text { No ownership or } \\
\text { recognition for } \\
\text { contributors. }\end{array}$ & $\begin{array}{l}\text { Aggregated data and } \\
\text { reports published for } \\
\text { members to use. } \\
\text { Compensation made } \\
\text { possible through member } \\
\text { fees and research } \\
\text { participation requirements. } \\
\text { No recognition mechanisms } \\
\text { in place other than } \\
\text { membership. }\end{array}$ & $\begin{array}{l}\text { Contributors are } \\
\text { compensated for the use of } \\
\text { information and data. } \\
\text { Content is tied to } \\
\text { contributors with referencing } \\
\text { workflows. } \\
\text { Communication capabilities } \\
\text { offer networking and joint } \\
\text { venture opportunities. }\end{array}$ \\
\hline $\begin{array}{l}\text { Technical } \\
\text { Simplicity }\end{array}$ & $\begin{array}{l}\text { Simple operation for } \\
\text { both browsing and } \\
\text { contributing through } \\
\text { the internet. }\end{array}$ & $\begin{array}{l}\text { Simple operation for both } \\
\text { browsing and contributing } \\
\text { through the internet. Data } \\
\text { is automatically aggregated } \\
\text { and published. }\end{array}$ & $\begin{array}{l}\text { High degree of automation } \\
\text { using workflows simplifies } \\
\text { use, and formatting freedom } \\
\text { reduces technical } \\
\text { requirements. } \\
\text { Implement through internet. }\end{array}$ \\
\hline
\end{tabular}


Coreworx solutions were chosen for the development and implementation of the data management and sharing model presented in this thesis for a number of reasons: (1) their experience and capabilities with respect to developing EPPMS solutions for mega construction projects worldwide; (2) their strong research ties to the University of Waterloo; (3) the convenient location of their head office in Kitchener, ON, which would enable timely support; and (4) their commitment to supporting the development of the ideas presented in this research by providing infrastructure and manpower and by hosting the entire project for three years on their servers through in-kind contributions and at no direct cost to the University of Waterloo.

\subsubsection{Objectives and Scope of the Developed EPPMS}

The purpose of the EPPMS solution developed through this research was to implement a document management system that would support both data fusion models and other research activities in the construction research community. The fusion engines presented in the previous chapters of this thesis would in practice have to be fed data through this database via workflows embedded in the overall data fusion workflow. Therefore, the key objectives of the developed model included the following:

- Rigorous data management and document control for research data, research documents, reports, and post-project analysis for data fusion purposes;

- Facilitation of the ability for members to collaborate on research projects with researchers from other universities around the world; and,

- Implementation of highly efficient and workflow-driven review processes to ensure the quality and security of the content while also promoting collaborative research.

With respect to the volume of data, the scope of the implementation was limited to the data captured throughout the phases of the research presented in this thesis, and in terms of users, to a number of researchers from the University of Waterloo, Carnegie Mellon University, HerriotWatt University, and the University of Texas as well as a number of industry partners at Aecon Construction and SNC Lavalin. For the original implementation, the number of users was capped at 50 , and the size of the database at $100 \mathrm{~GB}$. This was considered commensurate with the number and distribution of participants involved in a data fusion deployment for a large construction project. Thus, both applications are validated by the functionality of this system. 


\subsection{EPPMS Structure and Functionalities}

The structure of the EPPMS implemented in this research is shown schematically in Figure 6-2. The users see only the project portal, which for this particular implementation was the Coreworx environment. The project portal interacts with the three other main components of the system: the work flow engine, the data management system (DMS), and the viewer. The functionalities of all four components are presented in the following subsections.

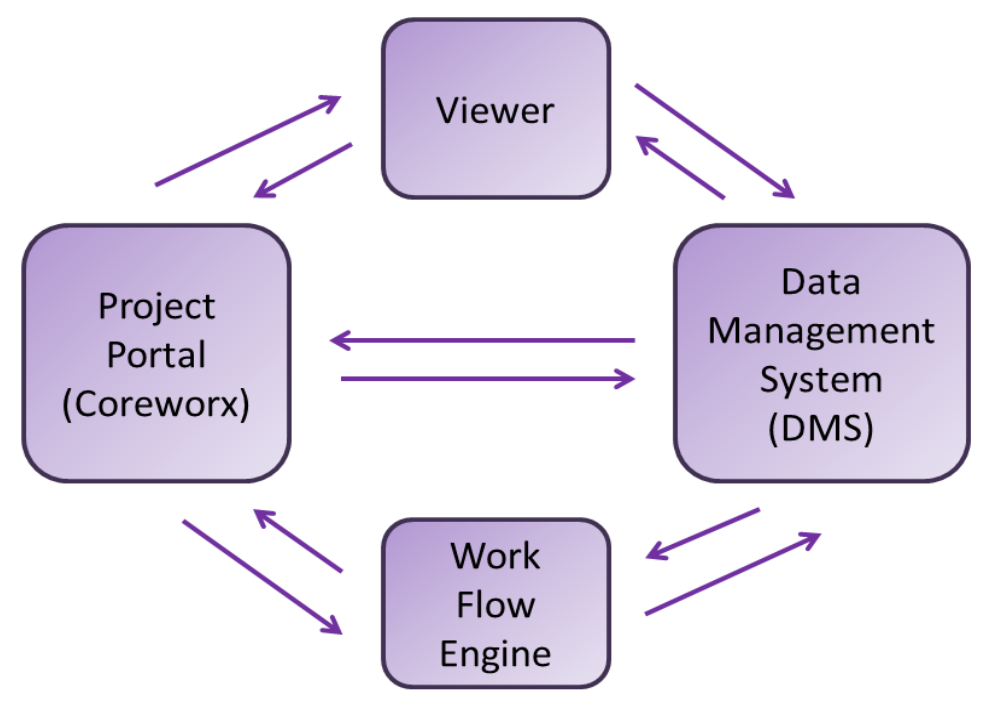

Figure 6-2: Structure of the Research Data EPPMS

\subsubsection{Web-Based Project Portal}

Figure 6-3 shows a snapshot of the project portal for the developed EPPMS solution. The portal is the user interface of the database system, which enables a secure login, project viewing, and the uploading and downloading of data. This module is completely web-based, thus requiring no software installation. The project portal provides a seamless interface to the DMS, the workflow engine, and the document viewer as the user navigates through the sections of the software. 


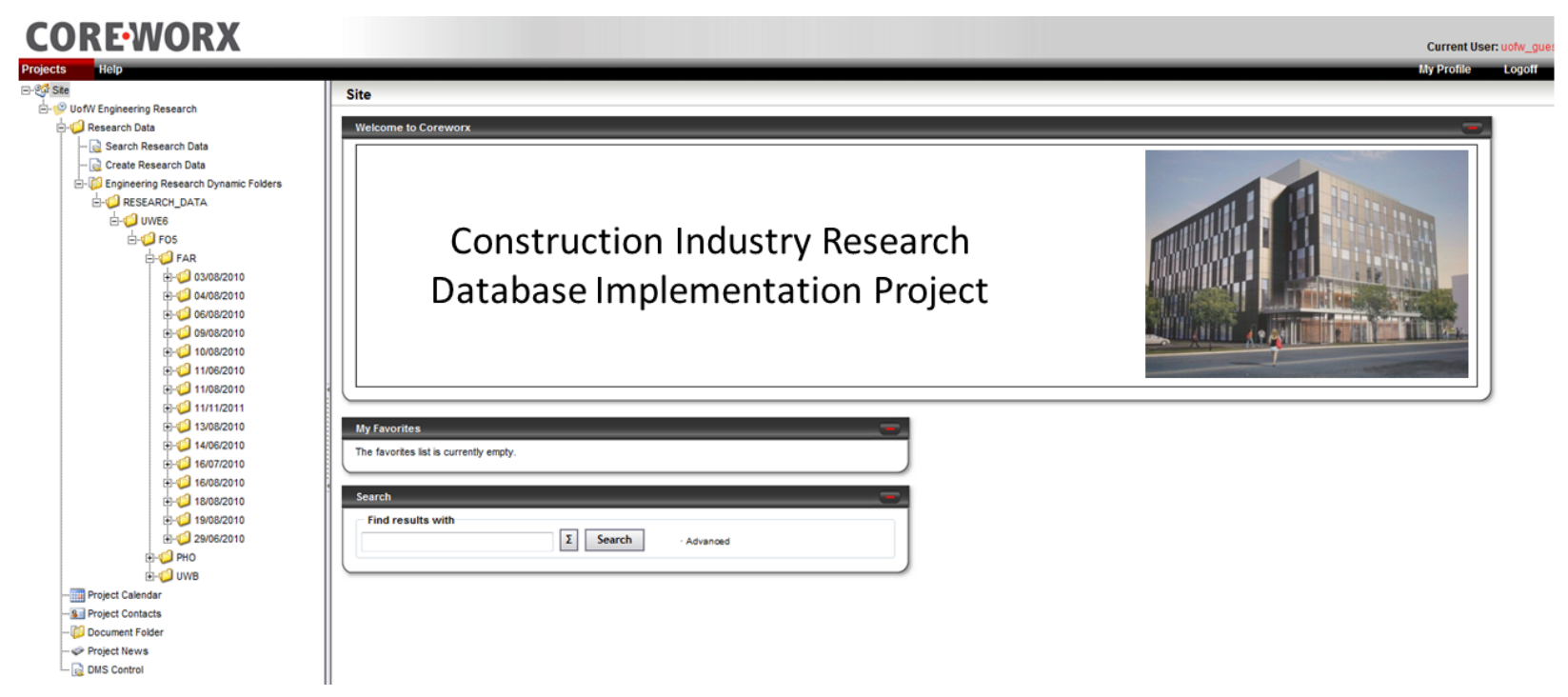

Figure 6-3: EPPMS Database Project Portal

\subsubsection{Document Management System (DMS)}

The Document Management System (DMS) is responsible for the control and management of the data. In the EPPMS solution presented in this thesis, the DMS is accessible through dynamic folders, as indicated on the left-hand side of the project portal shown in Figure 6-3. For data fusion processes, the required data would directly be withdrawn from the DMS using workflows. The DMS includes a number of functionalities that make it a viable solution for the management of research data: dynamic folders, document desktops, document administration, a related documents tool, document check-in and -out, and a search and retrieval process. These functionalities are explained in the following paragraphs.

\subsubsection{Dynamic folders}

Dynamic folders are located on the left-hand side of the user interface and provide a flexible and adjustable hierarchical viewing system for the metadata structure. These folders can be modified and customized for each research project, without modification of the underlying data structure, and allow users to find documents containing specific metadata values by navigating through a set of hierarchical nodes in the project tree. 


\subsubsection{Document Desktop}

Each user has access to a "document desktop," which aggregates the key documents and document workflows for which that user is responsible. A checked-out field, "favourite" documents (as identified by the user), and a "recently used documents" list provide users with fast, easy access to the key documents they've been involved with. This group of functionalities provides a very user-friendly and easy access to the documents stored in the DMS.

\subsubsection{Document Administration}

The EPPMS solution is not just an archiving system for completed documents, because it also contains all of the functions needed for the controlled administration of the research documents while they are being produced and processed. This particular functionality of the system is extremely valuable for collaborative research endeavours, including collaborative publications and data analysis. For example, the revision control feature captures changes to and histories of the documents, and access control ensures that classified information is available only to those who have the appropriate authorization. The use of access control can limit access to information for a research project to those working on the project, until the research is finalized, at which point it could be released in the system for all users to view.

\subsubsection{Related Documents Tool}

The EPPMS "related documents" tool has a wide range of applications in the research community domain and can be used to resolve some of the most important challenges with existing data-sharing and management models with respect to the particular application of sharing research data. The use of this tool enables documents in the database to be related to one another, which ensures explicit relationships that can then be used to identify source and successor information. In the developed research database, all research activities, including publications, that take advantage of a data source file must be "related" back to the source file. Although the performance of this step is based on an honour system, this functionality facilitates referencing tasks by ensuring that the original parties who collected the data are acknowledged for their work. Relating all consecutive research endeavors to the original data file(s) also ensure that all work related to the same data set is connected, which provides a very efficient and accurate collaboration tool. 
This functionality also helps to minimize the repetition of specific research tasks, which consequently improves the efficiency of the research. As a method of improving the effectiveness of this system, this feature also includes defined tags. For example, a "data input" tag is used for a "related document" if that document is considered to be the design basis or reference document used to create the current document, and a "data output" tag is used to indicate that a "related document" is the output of the current document.

\subsubsection{Document Check-In and -Out}

The checking in and out of documents within the developed EPPMS is another strength of this model for the particular application of research sharing and management. This functionality is not unique to EPPMSs and has already been implemented in a number of sophisticated webbased SharePoint systems. This tool ensures that only one copy of each source file is checked out at a given time, although simultaneous downloads may be permitted. In this context, "checking out" refers to a scenario in which one person has downloaded a document to edit or improve and is expected to upload or "check in" an improved version. This functionality does not refer to the data files, based on the use of which a number of research projects could be initiated simultaneously. Instead, this functionality is built in for collaborative projects, such as publications or for even collaborative data analysis scenarios, in which only one user is expected to make changes to a document during any given time period.

\subsubsection{Search and Retrieval}

The search and retrieval process within the EPPMS is highly customizable and very efficient, due primarily to the data structure and data profiles. The components of the data profiles are explained in detail in section 5.5 of this thesis, which describes the implementation and validation processes, because the data profiles were constructed specifically for the research project. Each data profile, which is used as the identity of each file within the system, is associated with a variety of mandatory drop-down menus and free-text fields, all of which could be used as search criteria. These metadata attributes and the dynamic folders presented earlier could also be used, either separately or in combination, as a means of searching for documents. 


\subsubsection{EPPMS Viewer}

The viewer component of the model, developed by Coreworx, interacts directly with the DMS and project portal. The viewer is one of the strengths of the EPPMS solution presented here because it allows the viewing of over 350 file types, from 3D CAD to Microsoft Office, without the requirement of the native application needing to be on the user's computer. This feature is particularly important for data files that must be viewed through specialty software, such as specific 3D point clouds from laser scanners, photogrammetric software, and BIM software, as long as the file size is manageable for the web browser. The viewer also permits the addition of markups in layers as well as links and notes, which are very useful for collaborative research.

\subsubsection{Workflow Engine}

The final component of the EPPMS solution presented in this thesis is the workflow engine. A core functionality of an EPPMS is the employment of workflows to control the flow of operations within the system. Workflows provide a formalized structure of the flow of the work or tasks to be performed during the execution of a process. Processes may therefore be described explicitly, and only members with predetermined responsibilities may execute work. Workflows can also incorporate temporal constraints, ensuring that work is completed within a reasonable timeline. While it is responsible for most of processes and actions performed by the software, the workflow engine operates in the background of the EPPMS and does not interact directly with users. The workflows that were implemented for the EPPMS solution presented in this thesis are described in the following section.

\subsection{Workflows for Construction Research Data Management}

In the construction research domain, a variety of workflows may be designed to assist with research-related activities. While some of these activities might already be possible in less formal environments such as journal paper review systems, the EPPMS provides the capability of efficiently structuring such processes while simultaneously enabling collaboration and access to the data that was used within the system for the research project in question. The following sections demonstrate some of the key workflows that were implemented in the developed EPPMS to facilitate the sharing and management of research data. 


\subsubsection{Review and Approval of Inbound Transmittals}

One of the major challenges with existing data-for sharing models for use in the construction research community is the lack of a systematic quality checking system and control of the materials that are added to the database. Data to be used in the data fusion model presented previously might be collected by any of a number of parties at the construction site, and the quality of the data that is being uploaded into the data management system and eventually into the data fusion model must be carefully controlled. The process-based EPPMS data management system developed in this research addresses this issue by using a "Review and Approval of Inbound Transmittals" workflow, shown schematically in Figure 5-3 and explained in detail in Table 6-2.

The documents to be uploaded are grouped based on their discipline and document type codes and are presented to the reviewers for commenting. It is important that each piece of information be reviewed prior to its inclusion in the research database. In this workflow, the resource approver selects three reviewers to examine the documents. Upon accessing the task from their Work Item inbox, the reviewers are presented with a comment cover sheet on which they can summarize their comments. Through that form, they have direct access to the documents to be reviewed as well as to any previous comments from others participating in that work process. The reviewer may also use the red-lining tool to mark up the file. If any one of the reviewers approves the data source and its quality, the data can be uploaded into the system. Three reviewers were selected primarily as a means of speeding up the process and providing enhanced flexibility. 


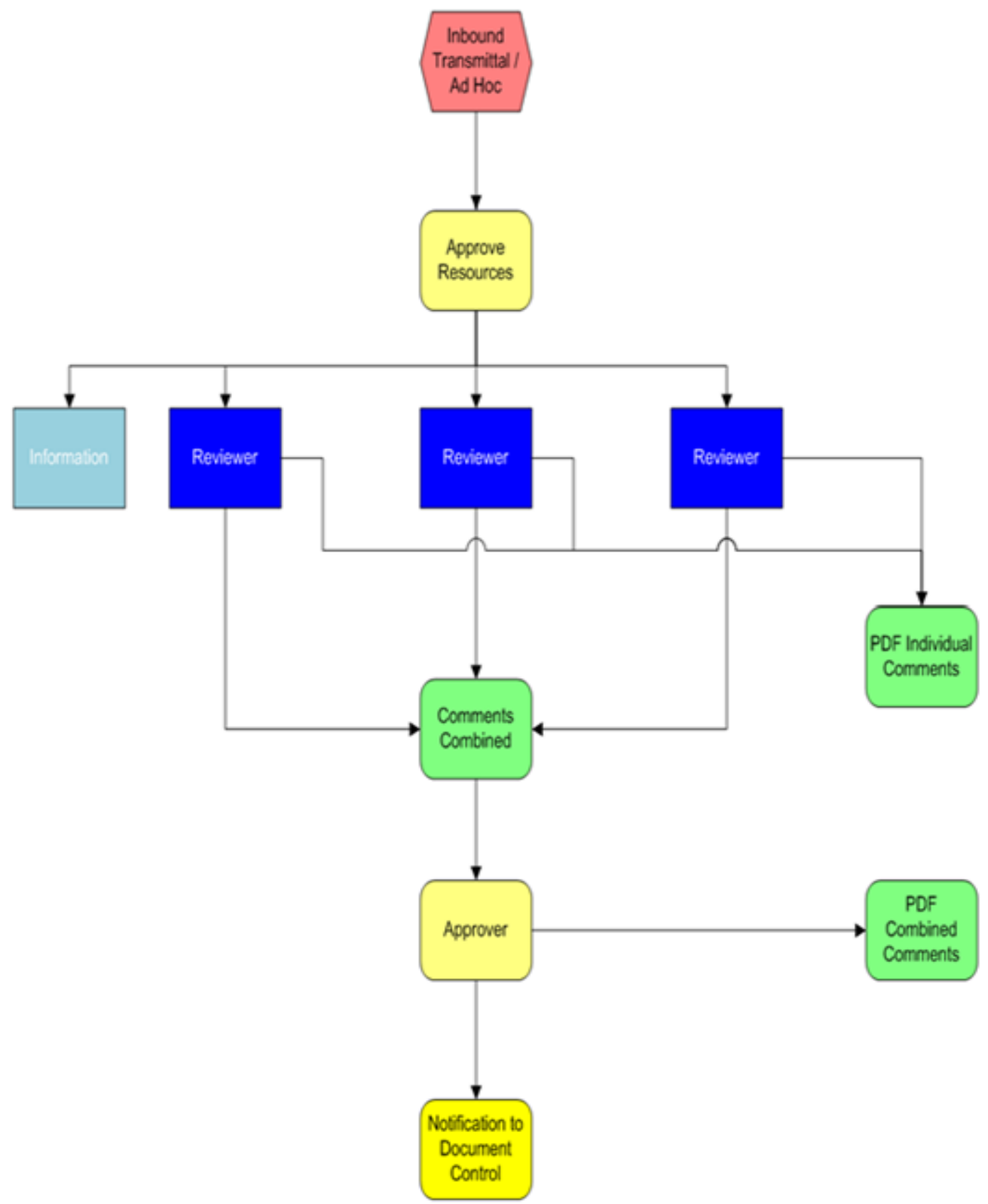

Figure 6-4: Review and Approval Workflow 
Table 6-2: Details of Main Activities within the Review and Approval Workflow

\begin{tabular}{ll}
\hline Activity & Description \\
\hline $\begin{array}{l}\text { User-Initiated } \\
\text { Workflow }\end{array}$ & $\begin{array}{l}\text { An authorized user may initiate the workflow. Using the Document } \\
\text { Cart, the user selects the documents to send into the workflow. }\end{array}$ \\
$\begin{array}{l}\text { Inbound } \\
\text { Transmittal }\end{array}$ & $\begin{array}{l}\text { One method of initiating the workflow is the receipt of Documents } \\
\text { from an external party via an inbound transmittal. The inbound } \\
\text { transmittal process takes all validated Documents and loads them to } \\
\text { the appropriate document archive, along with the metadata. }\end{array}$
\end{tabular}

Resource Assignment

Review(s)

Comment Sheet Consolidation
All validated Documents are grouped into logical data packages that are sent to a common group of reviewers and approvers. The resource assignment rules are maintained in the Responsibility Matrix for the project, which defines the Work Groups who will be the reviewers and approver to be assigned based on selected document attributes such as type of data, project, date, etc. For each inbound transmittal, the objective is to minimize the number of data packages that are created and sent on to a Review \& Approval workflow. Situations may arise in which a reviewer or approver is not defined in the Responsibility Matrix, which results in manual resource assignments that must be completed in a timely manner.

Each reviewer is responsible for conducting a review of the entire data package (usually multiple documents). For each document, the reviewers detail any issues, questions or comments they recommend for resolution using the electronic Comment Sheet. Individual Comment Sheets are maintained in the Administration archive.

All reviewer Comment Sheets are consolidated into one Comment Sheet for the data package. Once the sheets are completed, the approver is immediately sent his approval assignment. The comment sheets are stored in the Administration archive.
Approval
The approver makes a final determination of whether the data package will be
- Accepted - all documents are accepted as reviewed.
- Rejected - the external party (or internal team) must address the comments to the satisfaction of the approver before the data package is approved.

Outbound Transmittal
The Document Controller is notified that the data package is ready to be returned to the external party. Completed comment sheets are returned to the originator for resolution. The document profile for each Document listed on a Comment Sheet in the transmittal is updated with a status of either "Accepted" or "Rejected. 
This workflow was adjusted to accommodate other applications, such as collaborative publications, for which all three reviewers must approve a document before it passes through the approval process. However, for the uploading of raw data into the system, only one reviewer was deemed sufficient. In the existing state of the software, the resource approver's task is manual, but that task could also be automated through the automatic selection of the reviewers based on the type of data being uploaded. Once the comment sheets have been completed by the reviewers, they are merged into a single document (sheet) where the approver adds his or her comments. All comment sheets are stored in the DMS and are related back to the documents reviewed. The feedback from the reviewers is sent to the approver, who makes a decision to reject the data package or approve it for uploading into the system. One of the advantages of a process-based system is that sub-processes can be defined within the main process. For example, receiving feedback within a reasonable time frame may be enforced by activating timed milestones that indicate when each reviewer must complete the workflow. Throughout the process, participants are notified via email that they have a task to execute and are also notified if they are late in responding to the request.

The review and approval workflow described above was implemented in the construction research EPPMS as a means of ensuring a high degree of integrity for all data submitted to the system and of making sure that the data adheres to the standards of quality expected for organized research data. This workflow is flexible, extensible, and scalable. It addresses several issues related to the organization and structure of research data which are currently handled only informally. Similar systems often depend on the person who is responsible for his or her own set of data, and even within the same research initiative, this approach may be highly unstructured. The assignment of tasks, including reviewers, is accomplished through the Responsibility Distribution Matrix, which is an association file for relating tasks to the users of the system. This matrix is explained in the next section.

\subsubsection{Responsibility Distribution Matrix}

Document-based workflow processes are assigned to resources according to a configurable responsibility matrix. This Microsoft Excel spreadsheet contains a matrix that relates key document attributes to workflow work groups included in the database. Document-based workflow processes rely on the responsibility matrix for the selection of appropriate resources 
for each workflow activity. The responsibility matrix is an updateable, restricted-access document. Figure 6-5 illustrates a subset of the responsibility matrix developed in this research. The complete responsibility matrix can be found in Appendix C.

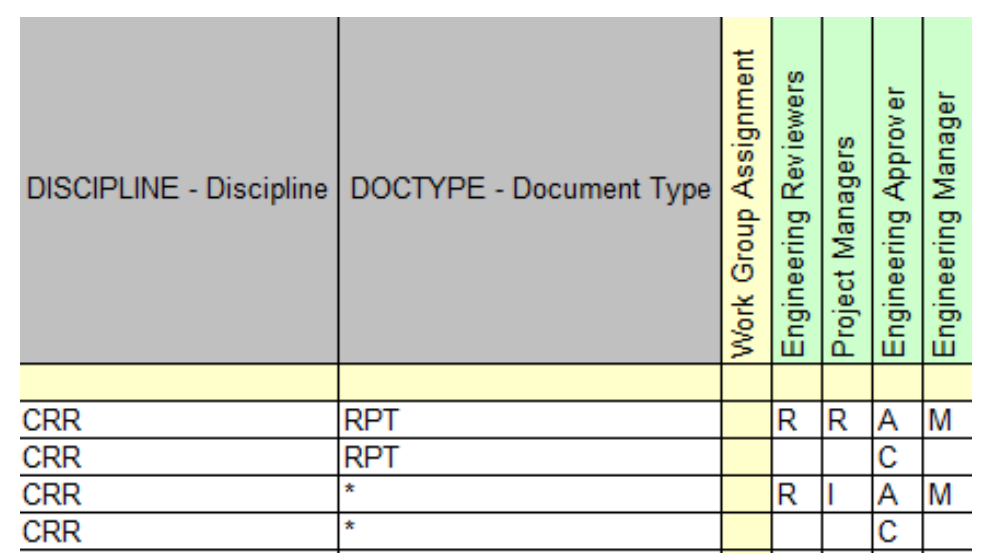

Figure 6-5: Sample Subset of a Responsibility Matrix

The work groups were defined during the configuration phase, and with the uploading of the matrix, are automatically configured in the system. The assignment of users to various work groups can be updated and modified at any time. In this example, documents are routed based on metadata values that indicate their discipline and document type. All of the work groups involved in review and approval are listed in the decision matrix, along with their role (approver, reviewer, consolidator, and informed). The "informed" role refers to a role that has no functionality requirements but that stays informed about the progress of the activities within the workflow. In the next section, this role is explained in greater detail in the context of the document notification workflow.

\subsubsection{Document Notification Workflow}

The Document Notification workflow process, shown schematically in Figure 6-6, is used to generate generic notifications to interested parties when new documents are received. The responsibility matrix is used to identify "informed" users. Alternatively, a user can manually initiate a document notification workflow and forward a notification to an individual user or to a work group. The "informed" role has a wide range of applications within the data management system developed in this research. For example, when users upload data, they can initiate the 
document notification workflow, which results in them to be informed and to receive notification if any other variations in the data or any publications using that data are uploaded to the database. The "related document" functionality, explained earlier, is responsible for determining the associations between the original data source and subsequent publications or data analysis results that are uploaded to the system.

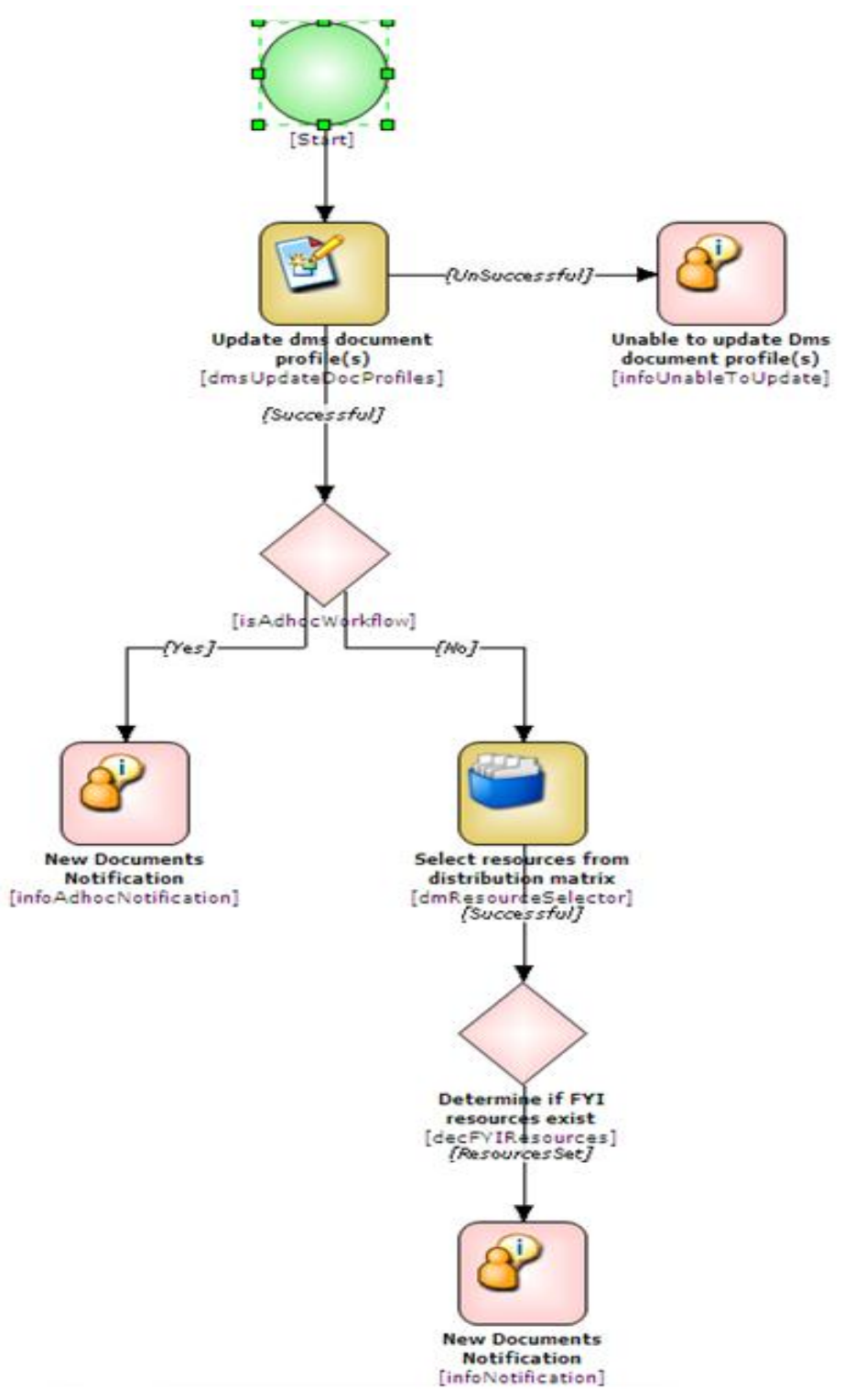

Figure 6-6: Document Notification Workflow 


\subsubsection{Assign Task Workflow}

The Assign Task workflow process is used for sending other research collaborators or project participants simple requests to complete the task indicated in the work item. This workflow is simply a formal request for a task to be completed, such as requests for scans to be completed, UWB data to be calibrated or uploaded, progress estimates to be submitted, etc. The request automatically shows up in the person's workflow inbox, in the order in which they were received; a formal log of all communications between the stakeholders on a project is thus maintained for the entire duration of the project.

\subsection{EPPMS Implementation}

This section summarizes the details of the implementation of the electronic product and process management system (EPPMS) that was developed in this research, using the data collected at the Engineering 6 building at the University of Waterloo throughout the research reported in previous chapters of this thesis. Three main steps are defined in this research for any project implementation of the developed EPPMS, as shown in Figure 6-7. The following subsections present the details of each step of the implementation process.

\section{Step 1: Original Hierarchical Data} Structure

Step 2: Specification of Metadata Attributes and Generation of a Flat File

Step 3: Bulk Upload to the Database

Figure 6-7: EPPMS Implementation Process 


\subsubsection{Step 1: Original Hierarchical Data Structure}

Any research project is expected to have a specific hierarchical data structure for the organization and storage of the information collected. The data from the Engineering 6 building was originally stored in a local database using a hierarchical structure, as shown in Figure 6-8. The data was structured according to the floor (or area), then by type of data (FARO Scans, UWB, Photogrammetry, etc.), and finally by date. Data was collected from a total of five floors in the building and also from some exterior data collection points. Not all data types were available on all floors. For example, UWB data was available only from the fifth floor because that floor was the only one that had UWB sensors. Photogrammetry and laser scans were taken from all floors but with varying frequencies and with the main concentration of data coming from the fifth floor.

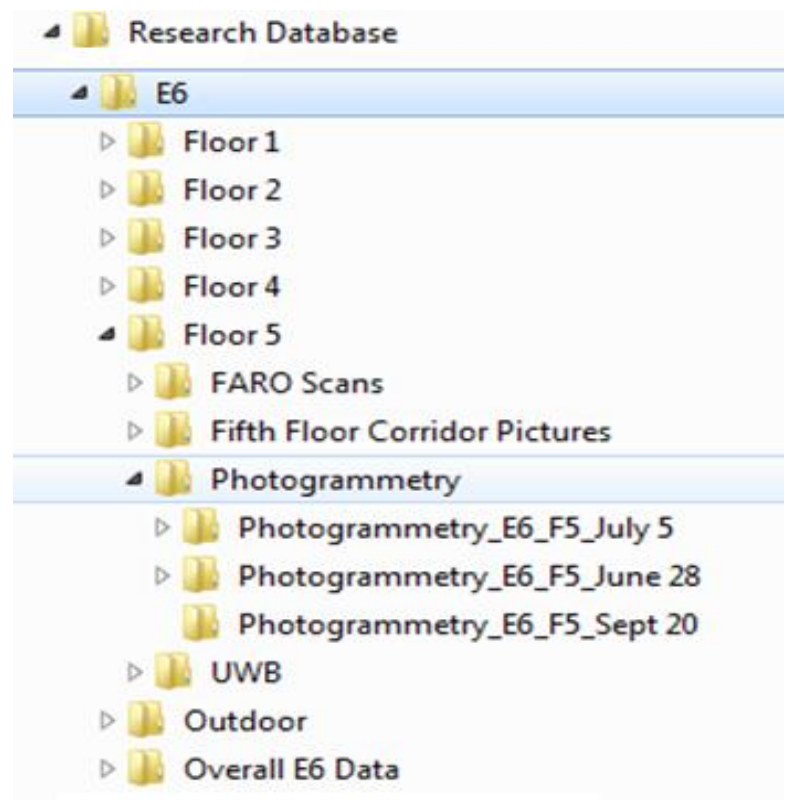

Figure 6-8: Original Hierarchical Structure of the Engineering 6 Data

More than 6000 individual data files were collected for this project. A substantial number of pictures were taken on each day and stored under "photogrammetry," which contributed significantly to the large number of data files. Because individual pictures would not be useful in any photogrammetric application and to reduce the load on the EPPMS application, all of the photogrammetry files collected on a specific day were zipped into one file, and each day would 
have only one associated photogrammetry file. This strategy reduced the total number of files for this project to 1164 .

Because the bulk uploading to the database requires that all files be located in one folder, any existing hierarchical data structure must be broken down, and all files should be moved to one main folder. This process necessitates each individual file having a distinct name since two files with the same name cannot be placed in one folder. The hierarchical structure can be used to specify the metadata structure and the attributes of the database using an associated flat file, as explained in the next step.

\subsubsection{Step 2: Specification of Metadata Attributes and Generation of a Flat File}

The hierarchical structure of the data, which was removed in the previous step, is recaptured in this step and translated into metadata attributes. The bulk upload process captures the metadata attributes using the flat file that is generated to accompany the files to be uploaded to the database. In this context, a "flat file" is a Microsoft Excel file that includes the file name, data attribute categories, and the corresponding metadata values for each file. A sample flat file for the University of Waterloo Engineering 6 building is shown in Figure 6-9. The metadata attributes are defined in Table 6-3.

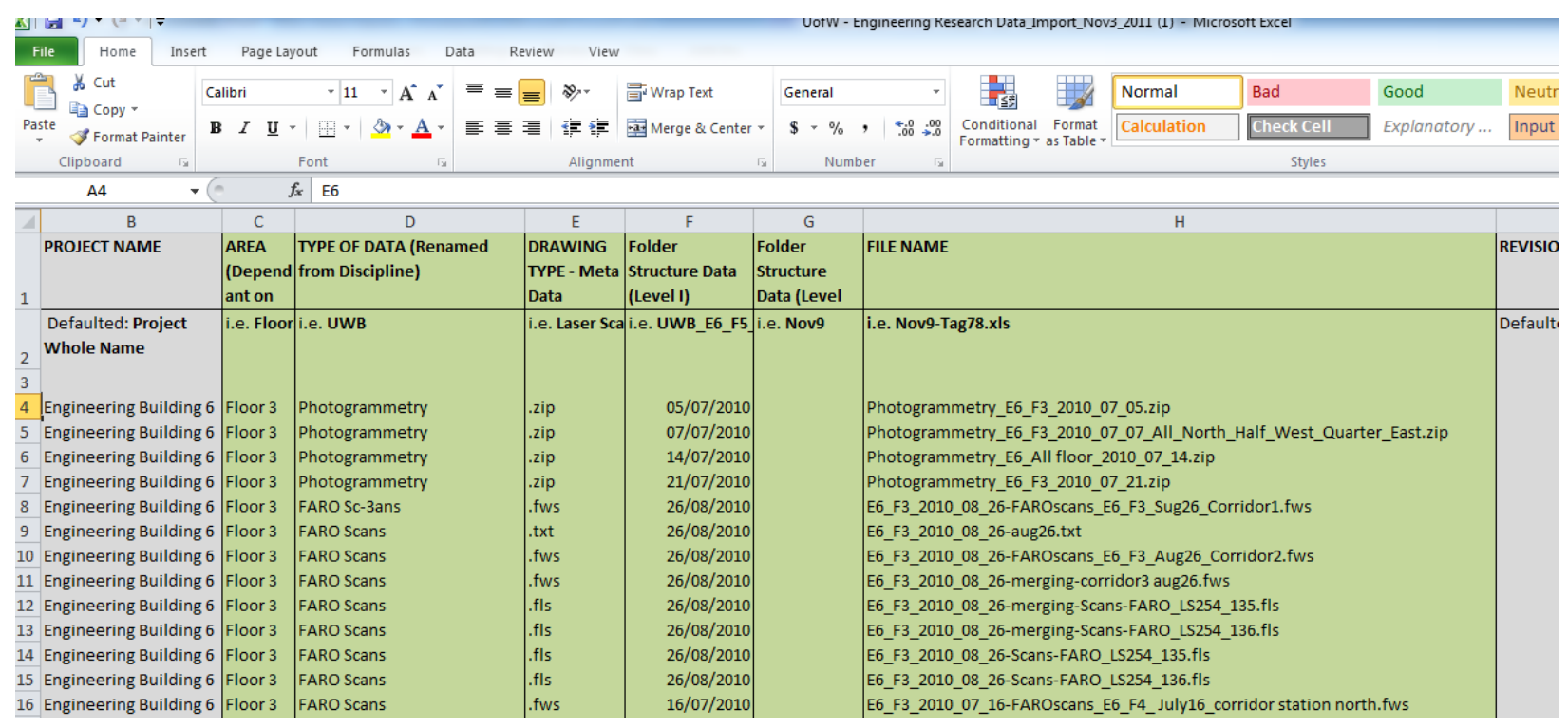

Figure 6-9: Sample Flat File for Engineering 6 Building Data 
Table 6-3: Metadata Attributes

\begin{tabular}{|c|c|c|c|}
\hline Attributes & Mand $\backslash O p t$ & \multicolumn{2}{|l|}{ Details } \\
\hline DOC \# & M & \multicolumn{2}{|c|}{$\begin{array}{l}\text { Automatically created during import: } \\
\text { PROJID( } 4 \text { char)-AREA( } 3 \text { char CODE)-TYPE( } 3 \text { char CODE)- } \\
\text { SEQ(5) } \\
\text { Example: UWE6-F05-UWB-00001 }\end{array}$} \\
\hline Project ID & $\mathrm{M}$ & \multicolumn{2}{|c|}{$\begin{array}{l}\text { Drop-down (4 characters alphalnumeric code) } \\
\text { Example: UWE6 }\end{array}$} \\
\hline Project Name & $\mathrm{M}$ & \multicolumn{2}{|c|}{$\begin{array}{l}\text { Drop-down (up to } 100 \text { characters) } \\
\text { University of Waterloo Engineering } 6\end{array}$} \\
\hline \multirow[t]{5}{*}{ Area } & $\mathrm{M}$ & \multicolumn{2}{|c|}{$\begin{array}{l}\text { Drop-down ( } 3 \text { characters alphalnumeric code) - Full name still } \\
\text { viewable in Description }\end{array}$} \\
\hline & & Code & Description \\
\hline & & F05 & Floor 5 \\
\hline & & F0x & Floor $\mathrm{x}$.. \\
\hline & & C01 & Floor 101 \\
\hline \multirow[t]{5}{*}{ Type of Data } & $\mathrm{M}$ & \multicolumn{2}{|c|}{$\begin{array}{l}\text { Drop-down ( } 3 \text { characters alphalnumeric code) - Full name still } \\
\text { viewable in Description }\end{array}$} \\
\hline & & Code & Description \\
\hline & & UWB & UWB \\
\hline & & FAR & FARO Scans \\
\hline & & PHO & Photogrammetry \\
\hline Description & $\mathrm{O}$ & \multicolumn{2}{|c|}{$\begin{array}{l}\text { Free text (up to } 25 \text { characters) } \\
\text { Could be used to add descriptions other than the predefined } \\
\text { metadata attributes }\end{array}$} \\
\hline Source Date & $\mathrm{M}$ & \multicolumn{2}{|c|}{$\begin{array}{l}\text { DATE type attribute } \\
\text { Refers to the data that the data was captured }\end{array}$} \\
\hline $\begin{array}{l}\text { Processing } \\
\text { Status }\end{array}$ & $\mathrm{M}$ & $\begin{array}{l}\text { Drop-do } \\
\text { Values: } \\
\text { DEFAL }\end{array}$ & \\
\hline Title & $\mathrm{M}$ & \multicolumn{2}{|c|}{$\begin{array}{l}\text { Automatically created during import using } \\
\text { PROJECT NAME(FULL) AREA(FULL) TYPE(FULL) } \\
\text { SOURCE DATE(FULL) } \\
\text { Example: University of Waterloo Engineering } 6 \text { Building Floor } \\
3 \text { Photogrammetry 14/JUL/2010 }\end{array}$} \\
\hline Revision & $\mathrm{M}$ & \multicolumn{2}{|c|}{ Defaulted to 1 (All files will be attached at revision level) } \\
\hline Status & $\mathrm{M}$ & \multicolumn{2}{|c|}{ Defaulted to IFR } \\
\hline Originator & $\mathrm{M}$ & \multicolumn{2}{|c|}{ Defaulted to UofW (4 Character String) } \\
\hline
\end{tabular}


The attributes defined in Table 6-3 correspond to the columns in the flat file. The second column in this table specifies whether each attribute is mandatory or optional. Currently, the only optional field is "Description," which is a free text attribute that could be used to capture any data attribute that is not built into the existing metadata structure. For example, for a building AutoCAD file, the Description could be used to explain the type of drawing (electrical, mechanical, structural, etc.), or for a zipped photogrammetry file, it could be used to identify how many pictures are included in the zipped file. Other attributes are defined as explained in the above table and are all mandatory. Once the flat file containing all the metadata attributes is generated, the files are ready for uploading to the database.

\subsubsection{Step 3: Bulk Upload Process}

There are two ways of uploading the files to the database: bulk upload and individual manual upload. The latter option requires each individual file to be uploaded separately and all attributes to be selected manually for each file upload through the document profile page, as shown in Figure 6-10. This process would be the normal procedure for uploading a file once a database for a particular project is up and running.

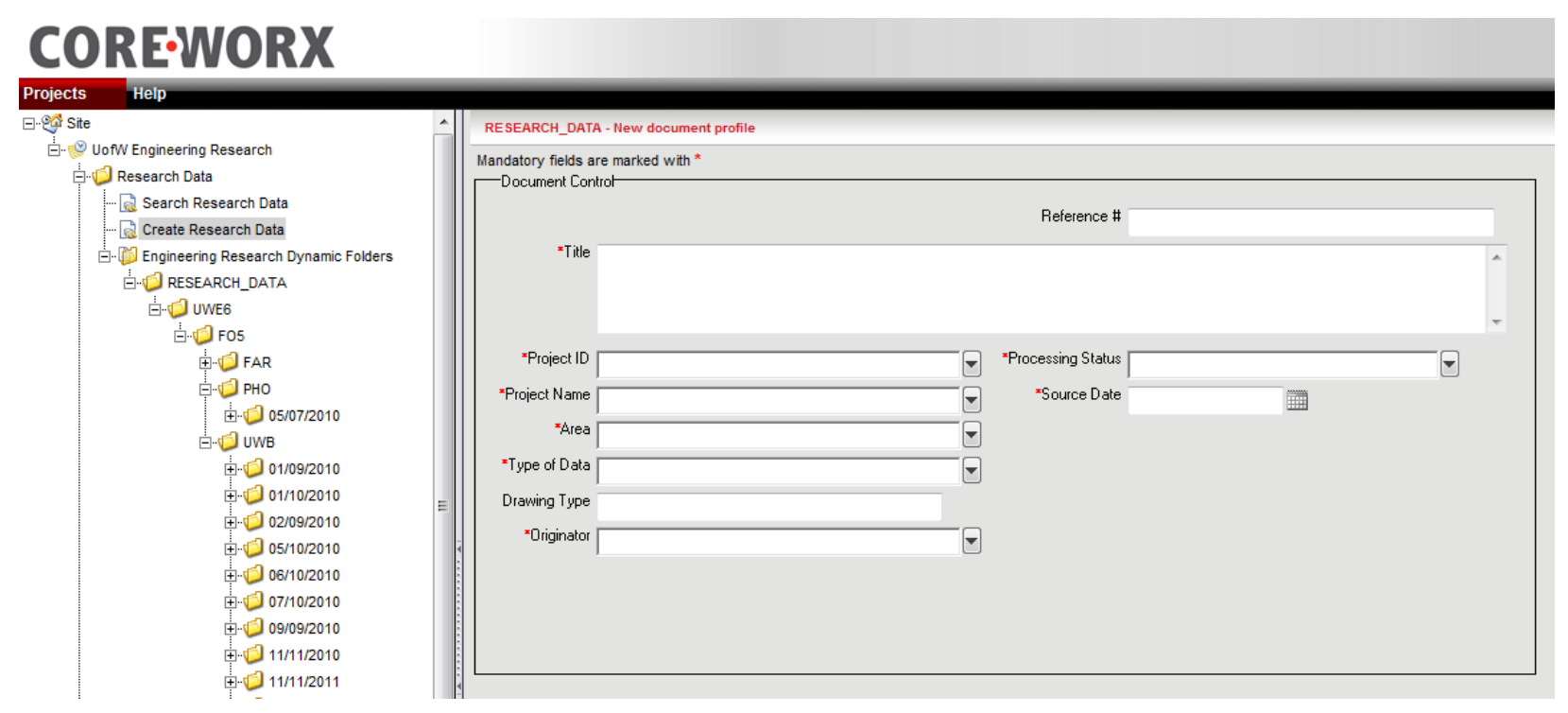

Figure 6-10: Document Profile for Manual Input

The manual upload option also requires each individual file to be subjected to the "Review and Approval of Inbound Submittal" workflow, which would require at least one reviewer to view 
each file and accept it for upload. With more than 1000 files, manual upload was not feasible, so a bulk upload process was used whereby all files were uploaded at the same time, bypassing the inbound workflow. In the case of a bulk upload, for each file to be uploaded, the database reads the file name and its corresponding metadata attributes from the Microsoft Excel flat file and automatically generates the document ID, file name, and other data file profile attributes. The bulk upload also enables the automatic renaming of the files using any combination of the metadata attributes and a sequence number to ensure unique naming. For the upload of the University of Waterloo Engineering 6 data, the following automatic naming system was used: PROJECT NAME(FULL) AREA(FULL) TYPE(FULL) SOURCE DATE(FULL). An example of an automatic name generation for an UWB files is University of Waterloo Engineering 6 Building Floor 5 UWB 09/SEP/2010. Figure 6-11 shows the screen capture fore opened document profile for this particular file.

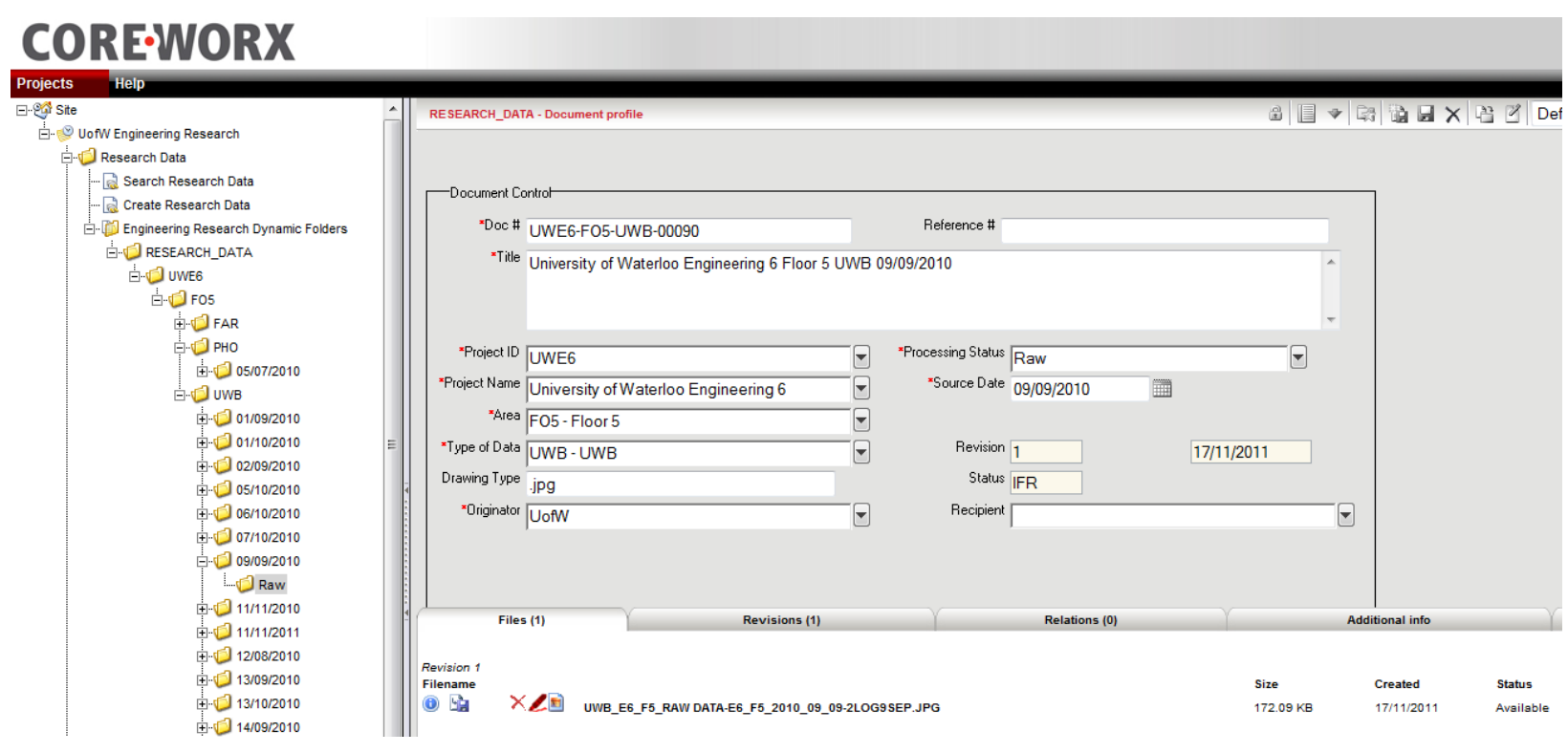

Figure 6-11: Sample Opened Document Profile

The successful upload of the files to the database represented the completion of the implementation of the University of Waterloo Engineering 6 research project. Those metadata attributes that were mandatory during the uploading process and were in the form of drop-down menus, as indicated in Table 6-3, were used to structure the dynamic folders, which were then used to navigate throughout the database. These menus were also used for the search engine 
of the system. Figure 6-12 presents a screen capture of part of the search results from a search conducted using search parameters: UofW project, Floor 5, and UWB.

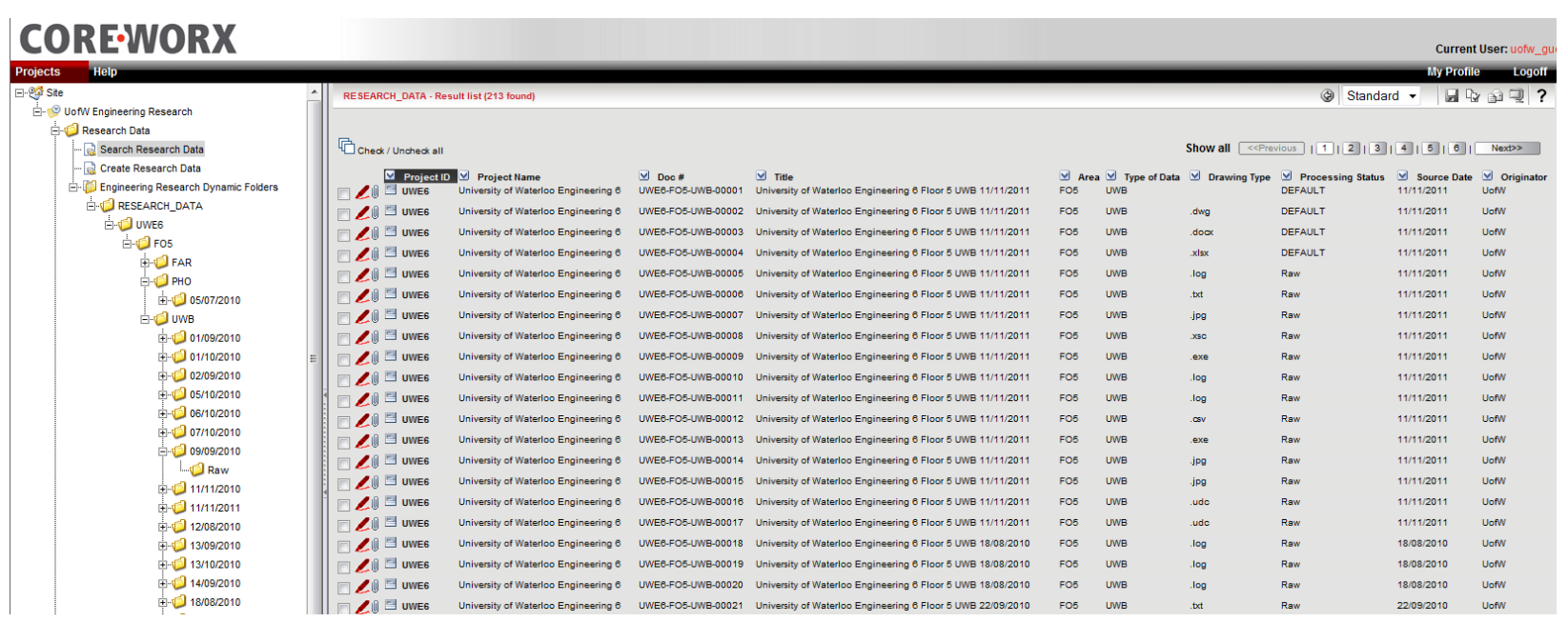

Figure 6-12: Sample Search Results of the Model

All of the functionalities developed for use in the DMS, such as the document desktop, document administration, the related documents tool, document check-in and -out, and the search and retrieval functions were operational as well. Finally, the workflows that were developed and implemented, such as "Review and Approval of Inbound Transmittals", "Document Notification" and "Assign Task" workflows were successfully verified based on the results reported from a number of researchers from the University of Waterloo, Herriot-Watt University, and Carnegie Mellon University, for whom user accounts were set up in the system.

\subsection{Integrated Data Fusion Workflow within EPPMS Framework}

The previous sections of this chapter have illustrated the successful implementation of the workflow driven data management system of this research for the construction industry, with an emphasis on storing and sharing data between the various stakeholders in the construction research community. In addition to addressing the current need in the construction research community for a reliable and efficient research data management system, the EPPMS framework presented in this chapter is also compatible with the data fusion workflows that were developed and presented in Chapter 5 of this thesis. The workflow based data fusion model of this research was previously illustrated in Figure 5-14 using Skelta framework, which is also the 
workflow engine for the EPPMS solution presented in this chapter. Therefore, future implementation and commercialization of the current research can consider incorporating the entire data fusion model of this research as a workflow within the developed EPPMS framework. This workflow would then extract its required data from the EPPMS database instead of the current file upload process explained in Appendix A. This integration would allow for web-based project control applications including automated construction progress tracking, automated scheduled updating, and automated earned value tracking from the EPPMS solution used for managing the data on a construction site.

Other applications of the integrated data fusion and management system for the construction industry include conflict resolution and negotiations. Conflicts between different stakeholders are common in the construction industry and a number of sophisticated models have been developed for resolution and negotiation of these conflicts (Kassab, Hegazy, \& Hipel, 2010; Yousefi, Hipel, \& Hegazy, 2010). While the scope of the current research did not include conflict assessment or conflict resolution in the construction industry, the developed workflow driven data fusion and data management models, presented in Chapters 5 and 6 of this thesis respectively, can potentially provide a strong conflict resolution tool to complement the existing models. This tool would be backed heavily by accurate and reliable information about the construction project and its stakeholders, stored within the workflows of the models. For example, "assign task" workflow documents the communications between the stakeholders and the tasks that have been assigned to each party, while the automated progress tracking workflow can measure the progress of the activities or tasks at various points along a particular "assign task" workflow. This information can then be used to resolve conflicts between the parties involved pertaining to the amount of work completed or the tasks that were assigned by one stakeholder to the other.

\subsection{Summary}

As the range of technologies being employed on construction projects increases, an enormous amount of data is being collected automatically or semi-automatically, resulting in a strong demand for a reliable data management system. To reap the benefit from their complementary advantages, these various sources of data and information are also being employed in data fusion models, such as the one presented in Chapter 5. However, developing reliable, efficient, 
and effective data fusion models requires a comprehensive data management and sharing system which in itself is reliable, efficient, and effective. In other words, in most data fusion models, the quality and organization of the database may control the quality of the fusion model itself. From a more a global perspective, a number of funding agencies, such as the National Institute of Health (NIH), the National Science Foundation (NSF), and the National Institute of Justice (NIJ), are now requiring data management and data-sharing systems for the projects they support.

In the last decade and in response to these demands, a number of research data management systems have been developed, primarily by educational institutions. A review of existing datasharing models revealed that they are currently incapable of addressing the demands of the construction research community. This chapter has presented a workflow-driven and processbased electronic product and process management system (EPPMS) as a construction research data management and data-sharing solution. This chapter included a comparison of the developed model with existing models based on the promotion of collaboration, accessibility, content quality, contributor compensation, and technical simplicity. The powerful capabilities of the proposed workflow-driven EPPMS model were also demonstrated through the description of a number of workflows, including a "Review and Approval of Inbound Transmittals" workflow. The details of the successful implementation of the model using the data captured during the various phases of the research presented in this thesis were also presented. Overall, the developed EPPMS model presented in this chapter has proven to be a promising solution for the data-sharing and data management problem faced by the construction research community, and it is also capable of being expanded to include other research communities as well.

The developed EPPMS model presented in this chapter is compatible with the workflow based data fusion model presented in Chapter 5. Future research can incorporate the data fusion processes of this research as a workflow within the developed EPPMS model, which would then allow for web-based project control applications within the EPPMS, such as automated construction progress tracking, automated schedule updating, and automated earned value tracking. This integrated solution could also support other decision making processes, including conflict resolution on construction projects. 


\section{Conclusions and Future Work}

\subsection{Conclusions}

This thesis presented a workflow based data fusion and management framework for managerial applications in the construction industry, including automated construction progress tracking. The developed workflows were implemented using a distributed computing network and archived using a cloud-based architecture. This research effectively addressed the process management and data management components of the JDL data fusion model, which were not developed in the previous implementations of the model in construction related applications.

It was not the intent of this research to develop a fusion model that relies on the enormous power of computers to process raw data. Rather, this research has developed a fusion architecture that can provide a more global and practical perspective of progress on construction projects and that is capable of assisting with decision-making processes, based on the sources of data and information available at any given time. In this research, an innovative indicator-tag approach was developed and integrated with activity-based data fusion algorithms to track both structural and non-structural activities. The developed model incorporated data obtained from state-of-the-art material- and event-tracking technologies, including ultrawideband (UWB) positioning systems and 3D laser scanning, and demonstrated the ability to fuse data from non-sensory sources of information, such as a Building Information Model (BIM), schedule information, and payment and progress reports. The experimental results show that the new fusion model successfully addresses the challenges of fusing multi-sensor data by conducting fusion processes at the higher levels of data fusion rather than employing the lowlevel fusion of traditional algorithms. As well, greater efficiency and accuracy have been achieved with respect to a number of construction management applications because the data fusion in the new model is based on construction activities instead of on objects and materials, as is the case with traditional sensor-based fusion strategies.

The workflow driven data management system presented in this research was able to provide an effective, efficient, and reliable system for the management and sharing of the enormous amount of data that was collected during this research. The data management model presented in this research can potentially be expanded to include data from the entire construction 
research community. Workflows, strategies, and recommendations were created to enable the stakeholders and consultants involved in a construction project to use the developed data management system for data sharing and management. The new system can also be employed by other research institutions around the world, both for data fusion purposes and for further collaboration on other research endeavors. In conclusion, an efficient, accurate, reliable, and scalable data fusion model for the purposes of automated construction progress tracking was developed and demonstrated.

\subsection{Contributions}

This research has provided significant contributions to five major areas of study: (1) the use of state-of-the-art sensing and data collection technologies for construction projects, (2) construction activity progress tracking, with an emphasis on tracking non-structural activities, (3) Workflow-based data fusion for construction progress estimation, (4) implementing data fusion algorithms corresponding to higher levels of data fusion in the JDL model, and (5) research data sharing and management. This section provides a brief discussion of the specific contributions in these five areas.

1) State-of-the-art technologies for data collection on construction projects have been investigated and evaluated for the purposes of developing a new method of automated construction progress tracking. As part of this research a detailed evaluation was conducted on the performance of an ultra-wideband (UWB) positioning system for construction industry applications. The laboratory and field experiments conducted during this research have characterized the performance of the system in construction environments and demonstrated the capabilities of the UWB positioning system as a material- and activity-tracking tool for construction projects.

2) An innovative indicator-tag approach was developed for using UWB positioning system to track the progress of non-structural activities on construction projects. Activity-based tracking is necessary to quantify the progress of activities, which are not directly associated with the addition or removal of physical entities on a site, such as the welding or inspection of pipe-spools. The indicator-tag approach was used as a progress data source in the model presented in this research, which provided a unique dimension of 
site information that was not incorporated in the previous attempts at automating construction progress tracking.

3) In this research, a workflow based data fusion framework is developed for construction progress, quality and productivity assessment. The developed model is based on tracking construction activities as well as objects, in contrast to the existing models that are only based on tracking objects. The data fusion processes of this research provide more accurate and more reliable progress and earned value estimates for construction project activities in comparison to existing methods of progress tracking on construction projects and other published fusion algorithms. These workflow driven processes can be developed into industry foundation standards for data fusion on construction projects, which would establish industry-wide data collection and management systems as well.

4) Other contributions of this research to the body of knowledge related to data fusion include (a) the development of fusion algorithms that operate at the decision-making level (or the highest levels of data fusion) to complement and enhance traditional sensory level (or low-level fusion) algorithms; (b) the fusion of data from sensory sources such as RFID and UWB systems together with information from non-sensory sources such as inspection, schedule, and progress reports; (c) and the fusion of data based directly on construction activities rather than on objects or materials.

5) This research has contributed to the body of knowledge related to data management by resulting in the development of a reliable, efficient, and scalable data management system for management and sharing construction research data, which was verified using the vast quantity and variety of data collected for this research. The developed data management system enables the efficient and secure sharing of data between the different stakeholders involved in a construction project: researchers, consultants, engineers, managers, etc. The body of knowledge about construction research has also been expanded because the new data management system incorporates functionalities that facilitate effective data-sharing among construction researchers regardless of their location, and in fact, a number of researchers from universities around the world have 
already verified the effectiveness of the data management model developed through this research, including researchers from Herriot Watt and Carnegie Mellon Universities.

\subsection{Limitations}

The components of the activity-based fusion model developed through this research were designed and verified using laboratory experiments as well as field implementation in a building construction project in Waterloo. A data management and data-sharing system was also developed to facilitate the use of the large amount of data that was collected on this site. The following limitations were taken into account during the creation, validation, and implementation of the data fusion and data management models developed in this research:

1) No published literature was available with respect to data fusion architectures for activity-based fusion for the purposes of automated construction progress tracking. The goal of this research was to establish benchmarks and industry-accepted standards on which future work in this area could be based and against which it could be evaluated. This limitation forced the parallel and iterative development and implementation of the model, which resulted in many functionalities of the model being developed after the field implementation and being based on the performance of the data collection techniques on site. The model was therefore validated in part through field experiments and in part through simulations performed based on the data collected on site. A full real-life implementation of the model is suggested for future work, during which final calibrations or design changes to the model could be completed.

2) The field implementation and validation of the fusion algorithms were further limited because they were restricted to the piping activities in an industrial-type building project. The piping systems were chosen because they include both structural activities directly associated with physical entities, such as delivery and installation, and other nonstructural activities, such as welding and inspection. A number of algorithms may need to be modified or other strategies employed so that all activities on a construction project can be included. The scope of this research was limited to piping activities as a means of demonstrating the feasibility of the proposed methodology for activity-based data fusion for automated construction progress tracking. 
3) The data management system that was developed in this research proved to be an effective, efficient, and reliable system for the purposes of data extraction for data fusion models. It was also shown to be effective for data sharing among project stakeholders and research groups. The validation and implementation of the model was limited to fully trusted parties at only a few universities. Further reliability assessments, financial sustainability strategies, and more comprehensive workflows are required prior to the release of the model for use by the entire construction research community.

\subsection{Outlook and Future Work}

The research presented in this thesis has two main components: an activity-based data fusion framework for automated construction progress tracking using state-of-the-art data technologies, and a data management system for the management and sharing of construction research data. The following recommendations are suggested for future research pertaining to both of these components:

- With respect to the further development of the fusion model presented in this research, a full implementation of the model on a construction project is recommended, during which final adjustments and calibrations can be completed. The developed activity-based fusion structure was implemented only for piping activities in an industrial-type construction project. Now that the model is constructed and the fusion algorithms are in place, the activity-based fusion model could be expanded to include the entire scope and duration of a construction project.

- This research represents one of the first attempts to provide activity-based progress estimates for construction-related activities. Two construction-related applications that could benefit from the use of activity-based progress estimates were also investigated: automated schedule updating and earned value estimation. With the successful implementation of the activity-based fusion algorithms and the promising results obtained from this research, future work could include the study of these two applications as well as other applications that can now be implemented with greater efficiency and reliability through the incorporation of these activity estimates. 
- The material tracking conducted for this research was limited to the boundaries of the construction site. However, with the expansion of the fusion algorithms to include all activities at a construction project, including procurement, it may be necessary to expand the material tracking methodology up the supply chain to include the fabrication stage. Future research could investigate methodologies for tracking materials at higher levels of the supply chain for the purposes of activity-based data fusion and better supply chain visibility. This research employed UWB positioning technology for material and Activity Progress Tracking; however, for the purposes of tracking critical elements at higher levels of the supply chain, GPS units are recommended because they can track objects anywhere in the world. Once they arrived at the project site, they would be replaced with UWB tags. Alternatively, UWB tags could be installed at fabrication shops, and another means of tracking the progress of materials to the construction site could be developed.

- In the current research, the level of BIM integration was limited to the extraction of 3D information about pipe spools for the activity- and material-tracking fusion algorithms as well as for the automated 3D object recognition algorithms. However, with the extent of the data collected for the development of the fusion architecture created for this research, a number of feedback functionalities could be developed to enable communication with the BIM framework. For example, a large number of 3D laser scans were collected and could be used to generate as-built models that could then be communicated back to the BIM to enable quality and accuracy assessments, future maintenance tasks, and as-built model reconstructions.

- The implementation of the data management system presented in this thesis was limited to the data captured for the specific project related to this study. Future work should examine the expansion of the data management system to include the entire construction research community. Sharing raw or semi-processed data with the whole community will improve collaborative work and enhance the quality and impact of construction research. Other future work could investigate the opportunity to invite other research centres, such as the Construction Industry Institute (CII), to join this data- 
sharing endeavour, which would add substantial value for both the academic community and industry partners. 


\section{References}

Ahmed, M., Haas, C. T., \& Haas, R. C. G. (2011a). Toward low-cost 3D automatic pavement distress surveying: The close range photogrammetry approach. Canadian Journal of Civil Engineering, 1(14), 38.

Ahmed, M., Guillemet, A., Shahi, A., Haas, C. T., West, J. S., \& Haas, R. C. G. (2011b). Comparison of point-cloud acquisition from laser-scanning and photogrammetry based on field experimentation. Paper presented at the CSCE 3rd International/9th Construction Specialty Conference, Ottawa, Ontario.

Ahmed, M., Haas, C. T., Shahi, A., Aryan, A., West, J. S., \& Haas, R. C. G. (2011c). Rapid tracking of pipe-works progress using digital photogrammetry. Paper presented at the CSCE 3rd International/9th Construction Specialty Conference, Ottawa, Ontario.

Ahmed, M., \& Hegazy, T. (2008). Comparison among indoor location-based technologies for construction and infrastructure applications. Annual Conference of the Canadian Society for Civil Engineering 2008 - "Partnership for Innovation", Quebec City, QC. , 1446-455.

Aryan, A., Shahi, A., West, J. S., Haas, C. T., Haas, R. C. G., \& Caldwell, G. (2011). Evaluation of ultra-wideband technology for position location and progress tracking in indoor construction environments. Paper presented at the CSCE 3rd International/9th Construction Specialty Conference, Ottawa, Ontario.

Axelsson, A., \& Schroeder, R. (2009). Making it open and keeping it safe: E-enabled datadharing in sweden. Acta Sociologica, 52(3), 213-226. doi:10.1177/0001699309339799 
Bosché, F. (2010). Automated recognition of 3D CAD model objects in laser scans and calculation of as-built dimensions for dimensional compliance control in construction. Advanced Engineering Informatics, 24(1), 107-118. doi:10.1016/j.aei.2009.08.006

Bosche, F., \& Haas, C. T. (2008). Automated retrieval of 3D CAD model objects in construction range images. Automation in Construction, 17(4), 499-512. doi:10.1016/j.autcon.2007.09.001

Bosche, F., Haas, C. T., \& Akinci, B. (2009). Automated recognition of 3D CAD objects in site laser scans for project 3D status visualization and performance control. Journal of Computing in Civil Engineering, 23(6), 311-318. doi:10.1061/(ASCE)08873801(2009)23:6(311)

Bosche, F., Haas, C. T., \& Murray, P. (2008). Performance of automated project progress tracking with 3D data fusion. Annual Conference of the Canadian Society for Civil Engineering 2008 - "Partnership for Innovation", Quebec City, QC. , $1349-358$.

Bosche, F. N., \& Haas, C. T. (2008). Automated retrieval of project three-dimensional CAD objects in range point clouds to support automated dimensional QA/QC. Electronic Journal of Information Technology in Construction, 13, 71-85.

Caron, F., Razavi, S. N., Song, J., Vanheeghe, P., Duflos, E., Caldas, C., \& Haas, C. (2007). Locating sensor nodes on construction projects. Autonomous Robots, 22(3), 255-263. doi:10.1007/s10514-006-9720-1

Ceci, S. J. (1988). Scientists' attitudes toward data sharing. Science, Technology, \& Human Values, 13(No. 1/2), 45. 
Cheng, M. Y., \& Chen, J. C. (2002). Integrating barcode and GIS for monitoring construction progress. Automation in Construction, 11(1), 23-33. doi:10.1016/S0926-5805(01)00043-7

Cheng, T., Venugopal, M., Teizer, J., \& Vela, P. A. (2011). Performance evaluation of ultra wideband technology for construction resource location tracking in harsh environments. Automation in Construction, 20(8), 1173-1184. doi:10.1016/j.autcon.2011.05.001

Cho, Y. K., Youn, J. H., \& Martinez, D. (2010). Error modeling for an untethered ultra-wideband system for construction indoor asset tracking. Automation in Construction, 19(1), 43-54. doi:10.1016/j.autcon.2009.08.001

Construction Industry Institute. (2009). 2009 strategic plan. Austin, Texas: The University of Texas at Austin. Retrieved from http://www.construction-institute.org/strat2009.pdf

Construction Industry Institute. (2010). Membership. Retrieved August 13, 2011, from https://www.constructioninstitute.org/scriptcontent/member.cfm?section=aboutcii\&activesubsection=member

Construction Industry Institute. (2011). Cll benchmarking code of conduct. Retrieved August 13, 2011, from https://www.construction-institute.org/scriptcontent/bmmcode.cfm?section $=$ bmm

Dasarathy, B. V. (1997). Sensor fusion potential exploitation-innovative architectures and illustrative applications. Proceedings of the IEEE, 85(1), 24-38.

Denning, P., Horning, J., Parnas, D., \& Weinstein, L. (2005). Wikipedia risks. Communications of the ACM, 48(12), 152. doi:10.1145/1101779.1101804 
Eastman, C., Teicholz, P., Sacks, R., \& Liston, K. (2008). BIM handbook A guide to building information modeling for owners, managers, designers, engineers, and contractors. Hoboken, New Jersey: John Wiley \& Sons, Inc.

El-Diraby, T. E. (2010). Semantic representation of infrastructure interdependencies: Rethinking infrastructure design and management. Construction Research Congress 2010: Innovation for Reshaping Construction Practice, Banff, AB. 656-665. doi:10.1061/41109(373)66

El-Gohary, N. M., \& El-Diraby, T. E. (2010). Dynamic knowledge-based process integration portal for collaborative construction. Journal of Construction Engineering and Management, 136(3), 316-328. doi:10.1061/(ASCE)CO.1943-7862.0000147

El-Omar, S., \& Moselhi, O. (2010). Database driven application for automated tracking and control of construction projects. Annual Conference of the Canadian Society for Civil Engineering 2010, CSCE 2010, Winnipeg, MB. , 1402-411.

El-Omari, S., \& Moselhi, O. (2009a). Data acquisition from construction sites for tracking purposes. Engineering, Construction and Architectural Management, 16(5), 490-503. doi:10.1108/09699980910988384

El-Omari, S., \& Moselhi, O. (2009b). Data acquisition from construction sites for tracking purposes. Engineering, Construction and Architectural Management, 16(5), 490-503. doi:10.1108/09699980910988384

El-Omari, S., \& Moselhi, O. (2011). Integrating automated data acquisition technologies for progress reporting of construction projects. Automation in Construction, 20(6), 699-705. doi:10.1016/j.autcon.2010.12.001 
Ergen, E., Akinci, B., East, B., \& Kirby, J. (2007). Tracking components and maintenance history within a facility utilizing radio frequency identification technology. Journal of Computing in Civil Engineering, 21(1), 11-20. doi:10.1061/(ASCE)0887-3801(2007)21:1(11)

Fischer, B. A., \& Zigmond, M. J. (2010). The essential nature of sharing in science. Science and Engineering Ethics, 16(4), 783-799. doi:10.1007/s11948-010-9239-x

Ghanem, A. G., \& Abdelrazig, Y. A. (2006). A framework for real-time construction project progress tracking. Earth and Space 2006 - 10th Biennial International Conference on Engineering, Construction, and Operations in Challenging Environments, League City/Houston, TX. , 2006 112. doi:10.1061/40830(188)112

Giffels, J., Vollmer, S. H., \& Bird, S. J. (2010). Editors' overview: Topics in the responsible management of research data. Science and Engineering Ethics, 16(4), 631-637. doi:10.1007/s11948-010-9243-1

Golparvar-Fard, M., Feniosky, P. M., \& Savarese, S. (2009). D4AR-A 4-dimensional augmented reality model for automating construction progress monitoring data collection, processing and communication. Electronic Journal of Information Technology in Construction, 14, 129153.

Golparvar-Fard, M., Pea-Mora, F., Arboleda, C. A., \& Lee, S. (2009). Visualization of construction progress monitoring with 4D simulation model overlaid on time-lapsed photographs. Journal of Computing in Civil Engineering, 23(6), 391-404.

doi:10.1061/(ASCE)0887-3801(2009)23:6(391) 
Grace, T. P. L. (2009). Wikis as a knowledge management tool. Journal of Knowledge Management, 13(4), 64-74. doi:10.1108/13673270910971833

Gu, X., \& Taylor, L. (2003). Ultra-wideband and its capabilities. BT Technology Journal, 21(3), 56-66. doi:10.1023/A:1025154914298

Haas, C. (2006). A model for data fusion in civil engineering. Intelligent Computing in Engineering and Architecture, Volume 4200/2006, ISBN 978-3-540-46246-0, 2006, pp. 315-319.

Hegazy, T., \& Menesi, W. (2010). Critical path segments scheduling technique. Journal of Construction Engineering and Management, 136(10), 1078-1085. doi:10.1061/(ASCE)CO.1943-7862.0000212

Hall, D. L., \& Llinas, J. (1997). An introduction to multisensor data fusion. Proceedings of the IEEE, 85(1), 6-23.

Han, S., Lee, S., Hong, T., \& Chang, H. (2006). Simulation analysis of productivity variation by global positioning system (GPS) implementation in earthmoving operations. Canadian Journal of Civil Engineering, 33(9), 1105-1114. doi:10.1139/L05-124

Hantziagelis, S., \& McCabe, B. (2006). Benchmarking airport reconstruction projects. Canadian Journal of Civil Engineering, 33(12), 1571-1584. doi:10.1139/L05-095

He, R., Ghavami, M., \& Aghvami, H. (2007). Ultra wide-band (UWB) positioning routing in ad hoc networks. 18th Annual IEEE International Symposium on Personal, Indoor and Mobile Radio Communications, PIMRC'07, Athens. doi:10.1109/PIMRC.2007.4394014 
Huber, D., Akinci, B., Tang, P., Adan, A., Okorn, B., \& Xiong, X. (2010). Using laser scanners for modeling and analysis in architecture, engineering, and construction. 44th Annual Conference on Information Sciences and Systems, CISS 2010, Princeton, NJ. doi:10.1109/CISS.2010.5464818

Joseph, S. A., Adams, B. J., \& McCabe, B. (2010). Methodology for bayesian belief network development to facilitate compliance with water quality regulations. Journal of Infrastructure Systems, 16(1), 58-65. doi:10.1061/(ASCE)1076-0342(2010)16:1(58)

Kassab, M., Hegazy, T., \& Hipel, K. (2010). Computerized DSS for construction conflict resolution under uncertainty. Journal of Construction Engineering and Management, 136(12), 1249-1257. doi:10.1061/(ASCE)CO.1943-7862.0000239

Khoury, H. M., \& Kamat, V. R. (2009). Evaluation of position tracking technologies for user localization in indoor construction environments. Automation in Construction, 18(4), 444457. doi:10.1016/j.autcon.2008.10.011

Kim, C., Son, H., \& Kym, C. (2011). The effective acquisition and processing of 3D photogrammetric data from digital photogrammetry for construction progress measurement. 2011 ASCE International Workshop on Computing in Civil Engineering, Miami, FL. 178185. doi:10.1061/41182(416)22

Kim, K., Kim, G., Kim, K., Lee, Y., \& Kim, J. (2009). Real-time progress management system for steel structure construction. Journal of Asian Architecture and Building Engineering, 8(1), 111-118. doi:10.3130/jaabe.8.111 
Kiziltas, S., \& Akinci, B. (2005). The need for prompt schedule update by utilizing reality capture technologies: A case study. Construction Research Congress 2005: Broadening Perspectives - Proceedings of the Congress, San Diego, CA. 321-330.

Kiziltas, S., Akinci, B., Ergen, E., Tang, P., \& Gordon, C. (2008). Technological assessment and process implications of field data capture technologies for construction and facility/infrastructure management. Electronic Journal of Information Technology in Construction, 13, 134-154.

Kwon, S., Bosche, F., Kim, C., Haas, C. T., \& Liapi, K. A. (2004). Fitting range data to primitives for rapid local 3D modeling using sparse range point clouds. Automation in Construction, 13(1), 67-81. doi:10.1016/j.autcon.2003.08.007

Larsen, K. E., Lattke, F., Ott, S., \& Winter, S. (2011). Surveying and digital workflow in energy performance retrofit projects using prefabricated elements. Automation in Construction, 20(8), 999-1011. doi:10.1016/j.autcon.2011.04.001

Lee, S., Thomas, S. R., \& Tucker, R. L. (2005). Web-based benchmarking system for the construction industry. Journal of Construction Engineering and Management, 131(7), 790798. doi:10.1061/(ASCE)0733-9364(2005)131:7(790)

McFadzean, G. E. (2003). Heriot Watt University - code of good practice in research and processes for handling allegations of misconduct in research Heriot Watt University. Retrieved on May $23^{\text {rd }} 2011$ from http://www.hw.ac.uk.proxy.lib.uwaterloo.ca/hr/htm/policies/Code\%20of\%20Research\%20C onduct $\% 20(2) \% 206 \% 20$ Mar\%2003\%20meeting.doc 
McIntosh, K., \& McCabe, B. (2003). Risk and benefits associated with international constructionconsulting joint ventures in the English-speaking caribbean. Canadian Journal of Civil Engineering, 30(6), 1143-1152. doi:10.1139/103-063

Moon, S., \& Yang, B. (2010). Effective monitoring of the concrete pouring operation in an RFIDbased environment. Journal of Computing in Civil Engineering, 24(1), 108-116. doi:10.1061/(ASCE)CP.1943-5487.0000004

Motamedi, A., \& Hammad, A. (2009). Lifecycle management of facilities components using radio frequency identification and building information model. Electronic Journal of Information Technology in Construction, 14, 238-262.

National Institute of Justice. (2010). Applying the the data resources program solicitation: Funding for the analysis of existing data. Retrieved June 9, 2011, from http://www.nii.gov/nii/funding/data-resources-program/applying.htm\#head2

National Institutes of Health (NIH). (2003). NIH data sharing policy and implementation guidance. Retrieved June 9, 2011, from http://grants.nih.gov/grants/policy/data sharing/data sharing guidance.htm

National Science Foundation. (2011). Chapter VI - other post award requirements and considerations. Retrieved June 9, 2011, from http://www.nsf.gov/pubs/policydocs/pappguide/nsf11001/aag 6.jsp\#VID4

Navon, R., \& Sacks, R. (2007). Assessing research issues in automated project performance control (APPC). Automation in Construction, 16(4), 474-484. doi:10.1016/j.autcon.2006.08.001 
Pradhan, A., Akinci, B., \& Haas, C. T. (2011). Formalisms for query capture and data source identification to support data fusion for construction productivity monitoring. Automation in Construction, 20(4), 389-398. doi:10.1016/j.autcon.2010.11.009

Razavi, S. N. (2008). Data fusion for location estimation in construction. (Ph.D. Comprehensive Report, University of Waterloo).

Razavi, S. N., Haas, C. T., Vanheeghe, P., \& Duflos, E. (2009). Real world implementation of belief function theory to detect dislocation of materials in construction. 2009 12th International Conference on Information Fusion, FUSION 2009, Seattle, WA. 748-755.

Razavi, S. N., Young, D. A., Nasir, H., Haas, C., Caldas, C., Goodrum, P., \& Murray, P. (2008a). Field trial of automated material tracking in construction. Annual Conference of the Canadian Society for Civil Engineering 2008 - "Partnership for Innovation", Quebec City, QC. , 3 1503-1511.

Razavi, S. N., Young, D. A., Nasir, H., Haas, C., Caldas, C., Goodrum, P., \& Murray, P. (2008b). Field trial of automated material tracking in construction. Annual Conference of the Canadian Society for Civil Engineering 2008 - "Partnership for Innovation", Quebec City, QC. , 3 1503-1511.

Razavi, S. N., \& Haas, C. T. (2010). Multisensor data fusion for on-site materials tracking in construction. Automation in Construction, 19(8), 1037-1046. doi:10.1016/j.autcon.2010.07.017

Reagle, J. (2009). Wikipedia: The happy accident. Interactions, 16(3), 42-45. doi:10.1145/1516016.1516026 
Rodriguez, Y. A., \& Jaselskis, E. J. (1994). Automated timekeeping and project information system using bar coding techniques. Proceedings of the 38th Annual Meeting of AACE International, San Francisco, CA, USA.

Sacks, R., Navon, R., \& Brodetskaia, I. (2006). Interpretation of automatically monitored lifting equipment data for project control. Journal of Computing in Civil Engineering, 20(2), 111120. doi:10.1061/(ASCE)0887-3801(2006)20:2(111)

Sacks, R., Navon, R., Brodetskaia, I., \& Shapira, A. (2005). Feasibility of automated monitoring of lifting equipment in support of project control. Journal of Construction Engineering and Management, 131(5), 604-614. doi:10.1061/(ASCE)0733-9364(2005)131:5(604)

Seymour, C. (2007). Benefits of using GPS guidance on excavators and shovels. Engineering and Mining Journal, 208(3), 92-96.

Shahi, A., Aryan, A., West, J.S., Haas, C.T., and Haas, R.C.G. (2011). Deterioration of UWB Positioning During Construction. Automation in Construction (in press)

Shehab, T., Moselhi, O., \& Nasr, E. (2009). Barcode-assisted system for document management of construction projects. International Journal of Construction Education and Research, 5(1), 45-60. doi:10.1080/15578770902717592

Soibelman, L., Wu, J., Caldas, C., Brilakis, I., \& Lin, K. (2008). Management and analysis of unstructured construction data types. Advanced Engineering Informatics, 22(1), 15-27. doi:10.1016/j.aei.2007.08.011 
Song, J., Haas, C. T., Caldas, C., Ergen, E., \& Akinci, B. (2006). Automating the task of tracking the delivery and receipt of fabricated pipe spools in industrial projects. Automation in Construction, 15(2), 166-177. doi:10.1016/j.autcon.2005.03.001

Song, J., Haas, C. T., \& Caldas, C. H. (2007). A proximity-based method for locating RFID tagged objects. Advanced Engineering Informatics, 21(4), 367-376. doi:10.1016/j.aei.2006.09.002

Steinberg, A. N., Bowman, C. L., \& White, F. E. (1999). Revisions to the JDL data fusion model. Proceedings of SPIE - the International Society for Optical Engineering, 3719, 430-441.

Tang, P., \& Akinci, B. (2011). Formalization of workflows for extracting bridge surveying goals from laser-scanned data. Automation in Construction, doi:10.1016/j.autcon.2011.09.006

Teizer, J., Caldas, C. H., \& Haas, C. T. (2007). Real-time three-dimensional occupancy grid modeling for the detection and tracking of construction resources. Journal of Construction Engineering and Management, 133(11), 880-888. doi:10.1061/(ASCE)07339364(2007)133:11(880)

Teizer, J., Haas, C. T., Caldas, C. H., \& Bosche, F. (2006). Real-time three-dimensional object detection and tracking in transportation. Applications of Advanced Technology in Transportation - Proceedings of the Ninth International Conference on Applications of Advanced Technology in Transportation, Chicago, IL. 123-128. doi:10.1061/40799(213)21

Teizer, J., Venugopal, M., \& Walia, A. (2008). Ultrawideband for automated real-time threedimensional location sensing for workforce, equipment, and material positioning and tracking doi:10.3141/2081-06 
Tseng, S. M., \& Huang, J. S. (2011). The correlation between wikipedia and knowledge sharing on job performance. Expert Systems with Applications, 38(5), 6118-6124. doi:10.1016/j.eswa.2010.11.009

Turkan, Y., Bosche, F., Haas, C. T., \& Haas, R. (2011). Automated progress tracking using 4D schedule and 3D sensing technologies. Automation in Construction, (0) doi:10.1016/j.autcon.2011.10.003

University of Pittsburgh. (2009). University of pittsburgh guidelines on research data management. Pittsburgh, PA: University of Pittsburgh. Retrieved on May $23^{\text {rd }} 2011$ from http://www.provost.pitt.edu/documents/RDM Guidelines.pdf

Weil, V., \& Hollander, R. (1990). Sharing scientific data II: Normative issues. IRB; a Review of Human Subjects Research, 12(2), 7-8.

White, F. E., Jr. (1987). Data fusion lexicon. San Diego: Joint Directors of Laboratories, Technical Panel for C3, Data Fusion Sub-panel, Naval Ocean Systems Centre.

Yang, H. L., \& Lai, C. Y. (2010). Motivations of wikipedia content contributors. Computers in Human Behavior, 26(6), 1377-1383. doi:10.1016/j.chb.2010.04.011

Yang, H. L., \& Lai, C. Y. (2011). Understanding knowledge-sharing behaviour in wikipedia. Behaviour and Information Technology, 30(1), 131-142. doi:10.1080/0144929X.2010.516019 
Yousefi, S., Hipel, K. W., \& Hegazy, T. (2010). Attitude-based negotiation methodology for the management of construction disputes. Journal of Management in Engineering, 26(3), 114122. doi:10.1061/(ASCE)ME.1943-5479.0000013 


\section{Appendix A}

\section{Graphical User Interface (GUI) for the Developed Earned Value Tracking Software}

This appendix provides a step-by-step representation of the Graphical User Interface (GUI) of the "Earned Value Progress Tracking" software that was developed for this research. This software was coded completely in MATLAB environment. Appendix B presents the entire code for this software. The Data Fusion Graphical User Interface (GUI), as depicted in Figure A-1, allows simple and user-friendly interaction with the software.

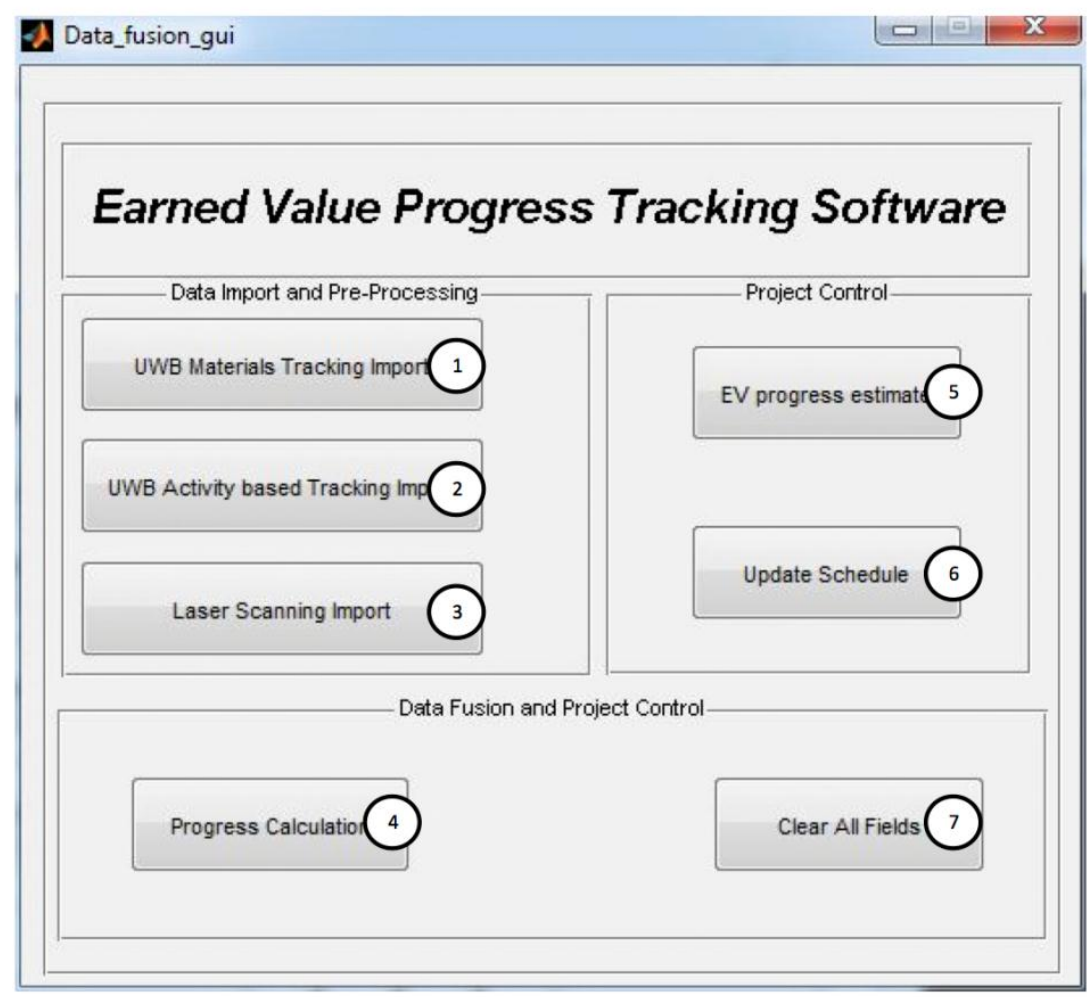

Figure A-1: The Graphical User Interface (GUI) 
Every process within the workflow, including the acquisition of user-input data, is accompanied by pop-up instructions that guide the user. Labels one through three indicate the data import and pre-processing procedures. Label four, the progress estimation, can be initialized after the first three are detected as complete. Labels five and six correspond to the project control tools within the GUI, as presented in Section 5.5.6 of this thesis. Label 7 clears all imported data, enabling the user to evaluate the progress of a different pipeline.

Figure A-2 illustrates the three stages for pre-processing and priming the raw data for data fusion processes within the model. First, the user initializes the materials tracking fusion, which is initially followed by a prompt for the user to select the 3D pipe information extracted from the 3D AutoCAD model. Next, the user is prompted to select the line to be analyzed and input the critical spools to be used later within materials tracking fusion algorithms. A sample screen capture of the prompts is shown in Figure A-3.

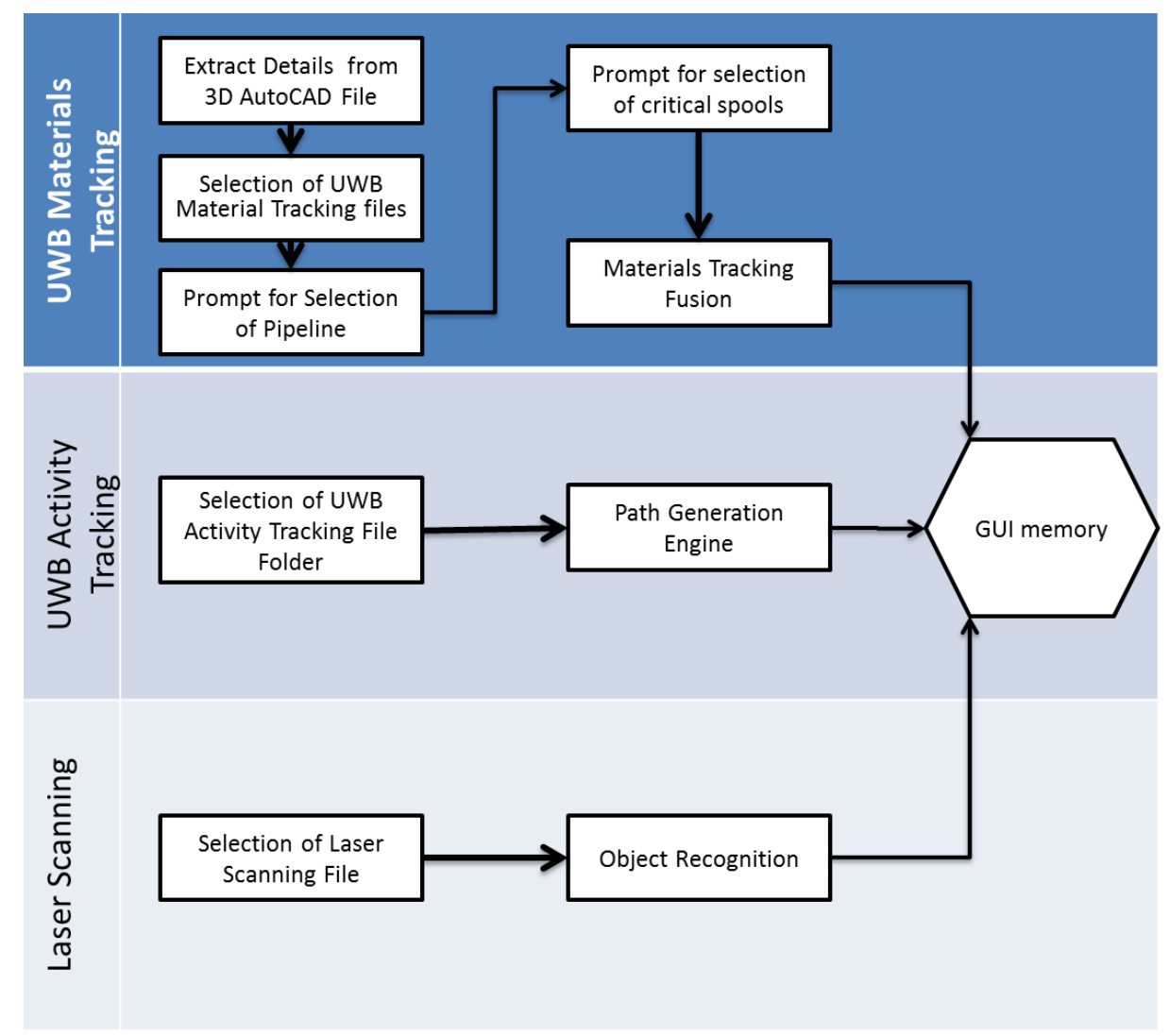

Figure A-2: Data Priming and Pre-processing Logic 


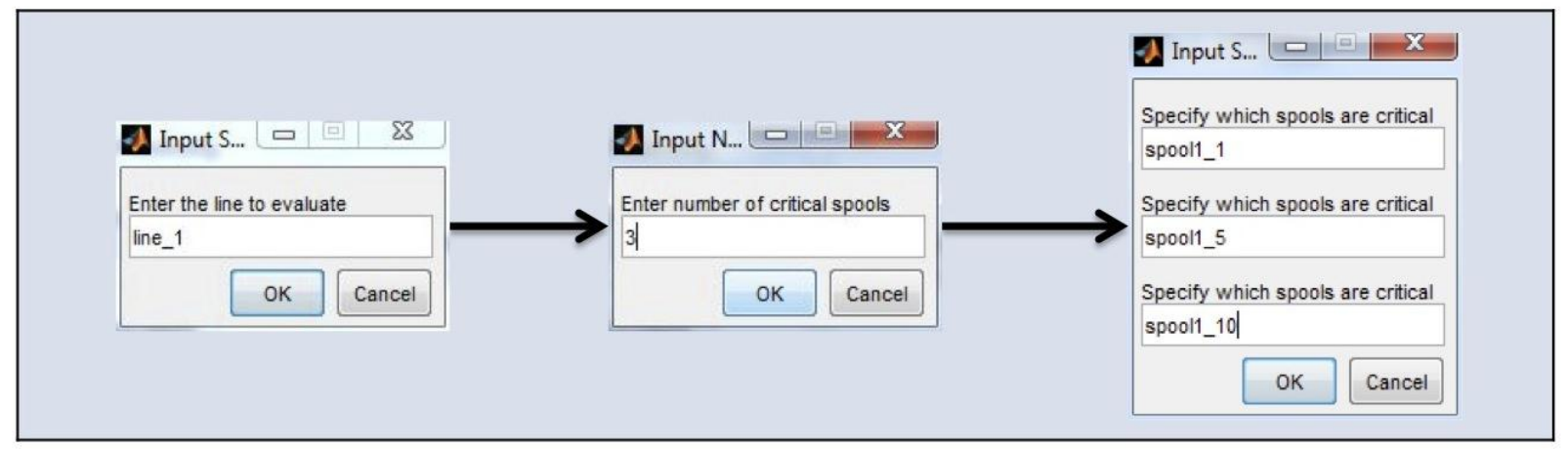

Figure A-3: Sequence of Prompts for Materials Tracking Fusion

UWB Activity Progress Tracking path generation follows the first stage. The user, following a prompt, selects the corresponding files. The file path is stored within the software for further analysis regarding the activity path generation. The Activity Progress Tracking paths are then generated using the established file formatting, as presented in Section 5.3 of this thesis and illustrated in Figure 5-6, and then stored within GUI memory for extraction during the activity based fusion process. Scanning is the final process within the initial data collection and preprocessing. A prompt indicates the user to select the file containing the object recognition results. These detection values are then stored within the GUI memory. The file must be consistent in format to the output that is extracted from the software developed by Bosche (2010).

Following the successful initial pre-processing of the data, the software enables the "Progress Calculation" functionality, which executes the workflow for fusion processes, explained in Chapter 5 of this thesis. Within the Progress Calculation functionality, the user is asked to identify the date and the pipeline for which the progress needs to be evaluated, through pop-up prompts as shown in Figure A-4. 


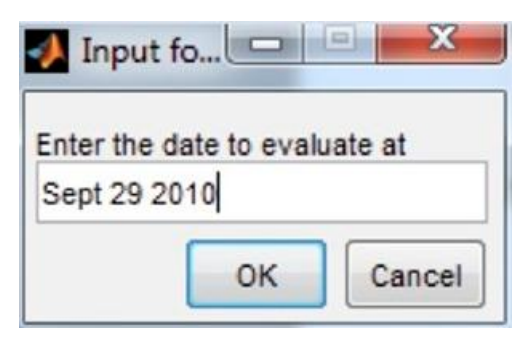

\section{Figure A-4: Progress Date Prompt}

The progress estimation results, once obtained, are stored within the GUI memory. The progress estimate is exported for the user in the way of an excel file, as shown in Figure A-5.

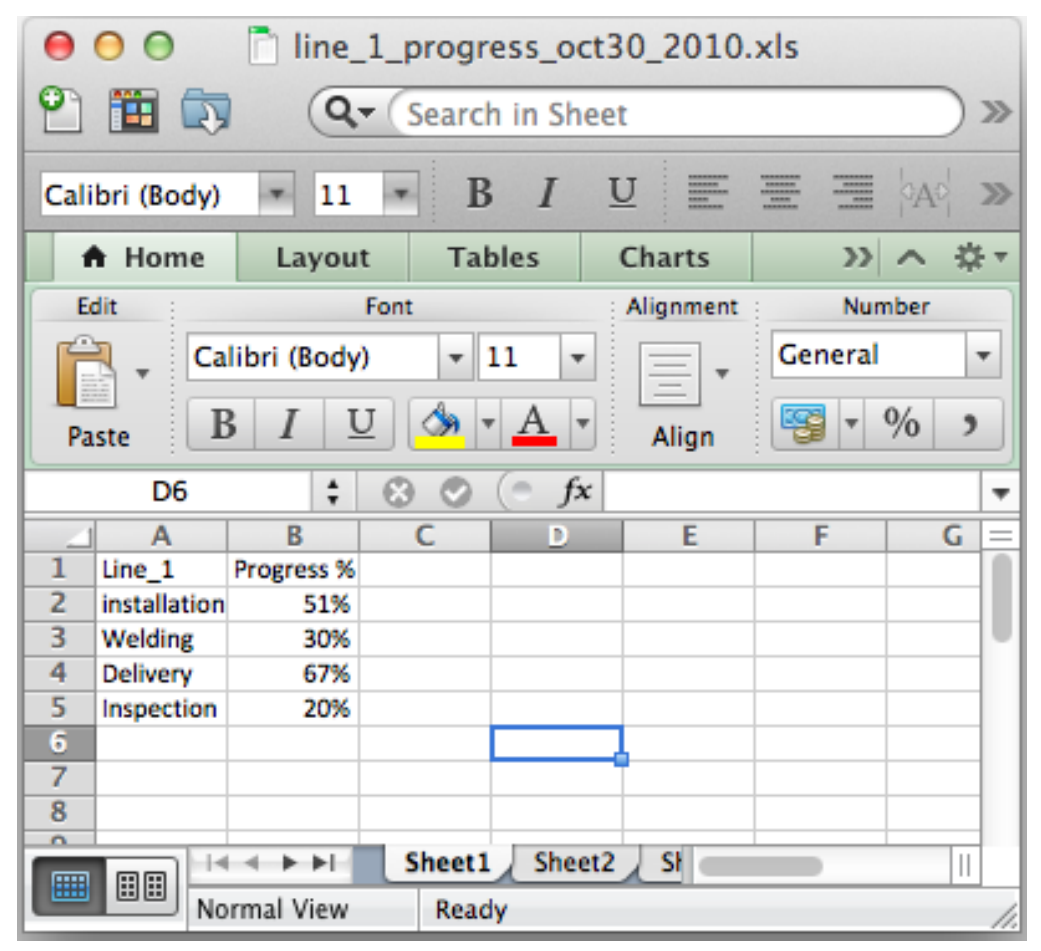

Figure A-5: Activity Based Fusion Output

Once the results from the Progress Calculation functionality are available, the software enables the Project Control functionalities, as indicated by numbers 5 and 6 on the GUI screen capture shown in Figure A-5. For schedule updating feature, a prompt appears to select the excel export of the original project schedule from Microsoft Project. A sample of an Excel export is shown in Figure A-6. Based on the progress calculated, the number of days required to finish an activity 
is re-evaluated. Figure A-7 illustrates a screen capture of an intermediary output of this function with the calculated new durations for each activity, which are then used to modify the original Excel export of the schedule. The updated schedule is then imported back to Microsoft Project, where the start and end dates of each activity are then recalculated based on the modified durations. The other project control feature installed within the GUI is the Earned Value estimate. The results of this feature of the software are presented in Section 5.5.6 of this thesis.

\begin{tabular}{|c|c|c|c|c|c|c|}
\hline \multicolumn{7}{|c|}{ Original Schedule } \\
\hline ID & Name & Duration & Start & Finish & Predecessors & Outline Level \\
\hline 1 & Line 1 & 36.0 & $21-09-20108: 00$ & $09-11-201017: 00$ & & 1 \\
\hline 2 & install & 29.0 & $21-09-20108: 00$ & $29-10-2010 \quad 17: 00$ & & 2 \\
\hline 3 & weld & $30 . d$ & $29-09-20108: 00$ & 09-11-2010 17:00 & & 2 \\
\hline 4 & inspection & $5 . d$ & $29-10-20108: 00$ & $04-11-201017: 00$ & & 2 \\
\hline 5 & Line 2 & 26.0 & $07-10-20108: 00$ & $11-11-201017: 00$ & & 1 \\
\hline 6 & install & 19.d & $08-10-20108: 00$ & $03-11-201017: 00$ & & 2 \\
\hline 7 & weld & $24 . d$ & $07-10-20108: 00$ & $09-11-201017: 00$ & & 2 \\
\hline 8 & inspection & $3 . d$ & $09-11-20108: 00$ & $11-11-201017: 00$ & & 2 \\
\hline 9 & Line 3 & 33.0 & $29-09-20108: 00$ & $12-11-2010 \quad 17: 00$ & & 1 \\
\hline 10 & install & 29.0 & 29-09-2010 8:00 & $08-11-201017: 00$ & & 2 \\
\hline 11 & weld & $15 . d$ & $25-10-20108: 00$ & $12-11-201017: 00$ & & 2 \\
\hline 12 & inspection & 1.d & $11-11-20108: 00$ & $11-11-201017: 00$ & & 2 \\
\hline 13 & Line 4 & 23.0 & $20-10-20108: 00$ & $19-11-2010 \quad 17: 00$ & & 1 \\
\hline 14 & install & 23.d & $20-10-20108: 00$ & $19-11-201017: 00$ & & 2 \\
\hline 15 & weld & $7 . d$ & $01-11-20108: 00$ & $09-11-201017: 00$ & & 2 \\
\hline 16 & inspection & 3.d & $03-11-20108: 00$ & $05-11-201017: 00$ & & 2 \\
\hline
\end{tabular}

Figure A-6: A sample project schedule without engine modifications.

\begin{tabular}{|c|c|c|c|c|}
\hline \multicolumn{2}{|c|}{$\begin{array}{c}\text { Progress Estimation Date } \\
2010-10-29 \\
\end{array}$} & & & \\
\hline & & & & \\
\hline Activity & Days Elapsed & $\%$ Accomplished & Remaining & New Duration \\
\hline Line_1_Installation & 29 & $51 \%$ & 15 & 44 \\
\hline Line 1 Welding & 23 & $30 \%$ & 21 & 44 \\
\hline Line_1_Inspection & 1 & $20 \%$ & 4 & 5 \\
\hline Line_2_Installation & 16 & $100 \%$ & 0 & 16 \\
\hline Line_2_Welding & 17 & $50 \%$ & 12 & 29 \\
\hline Line_2_Inspection & $=$ & $0 \%$ & $=$ & $=$ \\
\hline Line_3_Installation & 23 & $100 \%$ & 0 & 23 \\
\hline Line_3 Welding & 5 & $40 \%$ & 9 & 14 \\
\hline Line_3_Inspection & $=$ & $0 \%$ & $=$ & - \\
\hline Line_4_Installation & 8 & $24 \%$ & 18 & 26 \\
\hline Line 4 Welding & $=$ & $0 \%$ & - & $=$ \\
\hline Line_4_Inspection & $=$ & $0 \%$ & - & - \\
\hline
\end{tabular}

Figure A-7: Sample Duration Re-Calculations 


\section{Appendix B}

\section{MATLAB Code for Fusion Processes}

This appendix includes MATLAB code for the data fusion processes presented in Chapter 5 of this thesis. The code used to process the UWB analysis in Chapter 4 is not included in this appendix, as that code is not part of the "Earned Value Progress Tracking" software, developed as part of this research. Specifically, this appendix includes the code related to the Graphical User Interface (GUI), presented previously in Appendix A, and the code related to the MATLAB functions that were programmed for fusion processes, presented in Sections B1 and B2, respectively.

\section{B1. GUI Code}

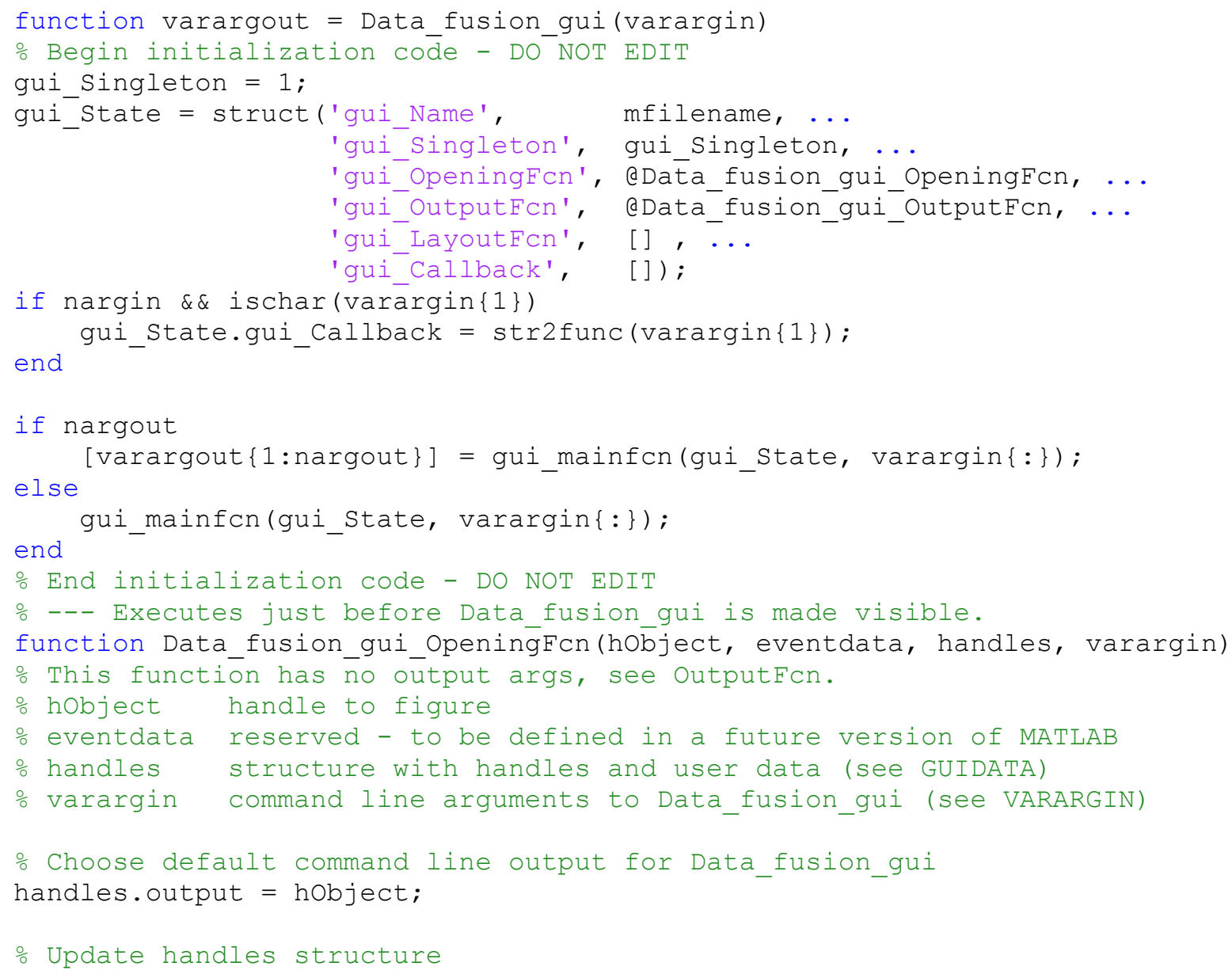




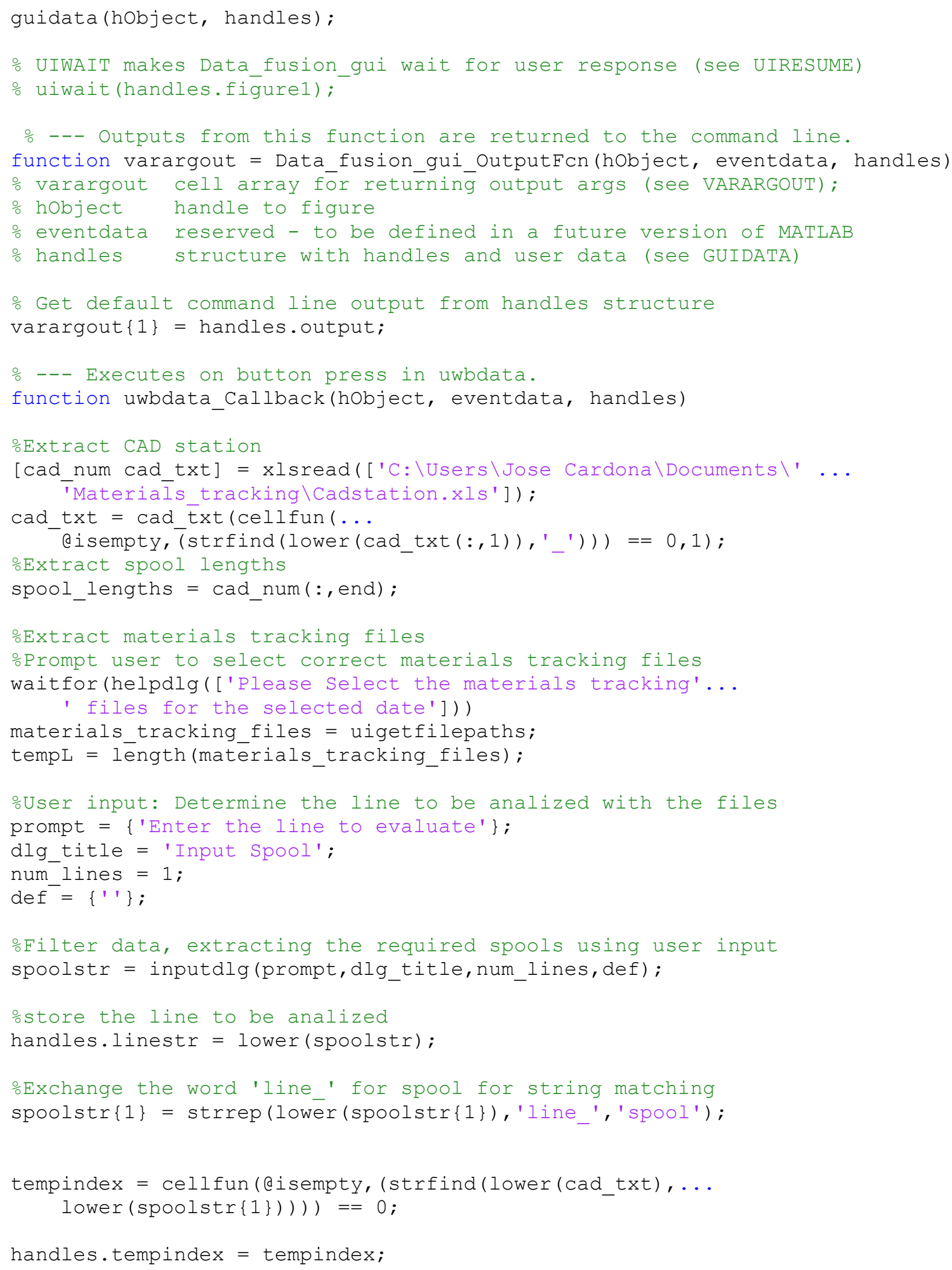




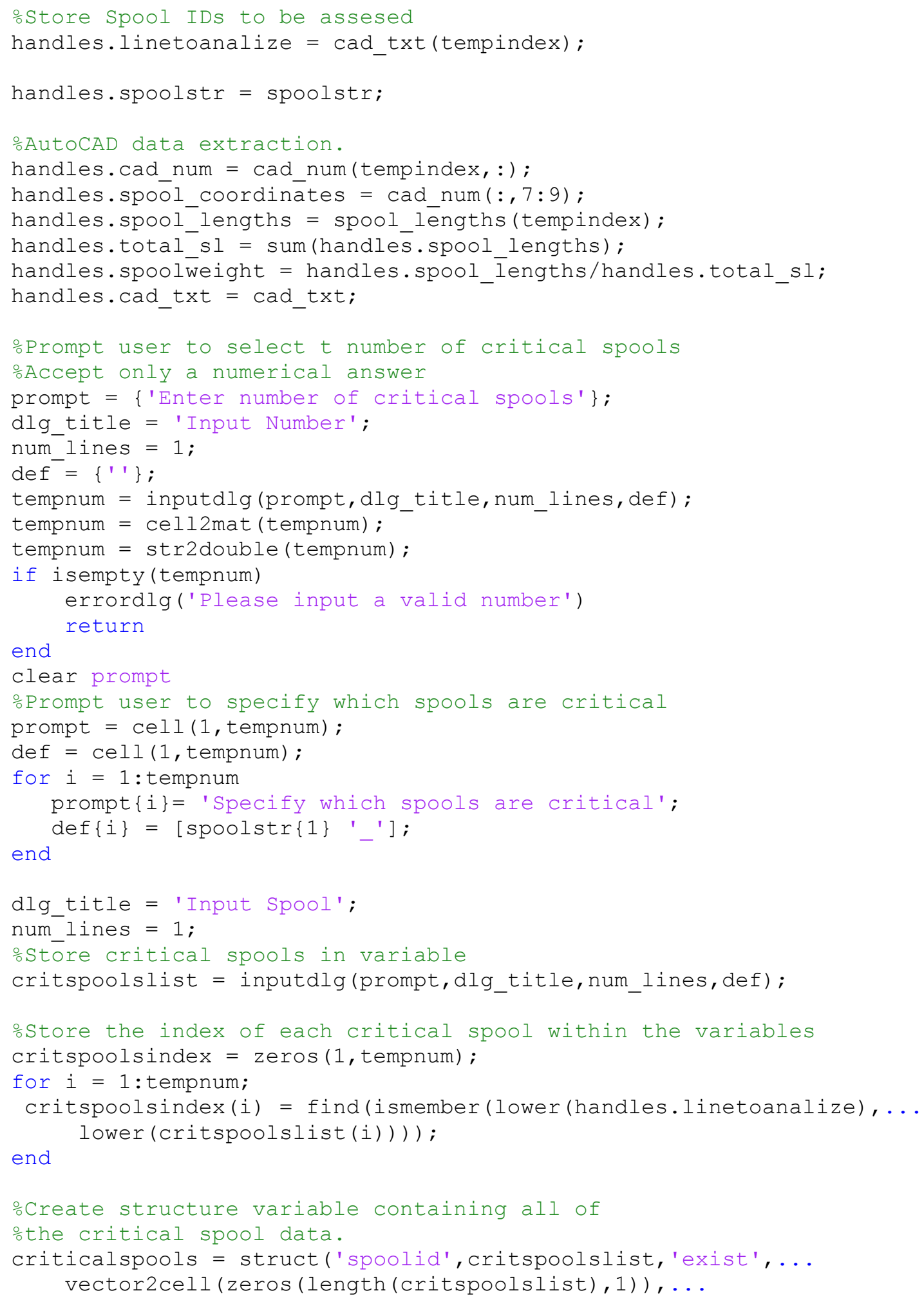




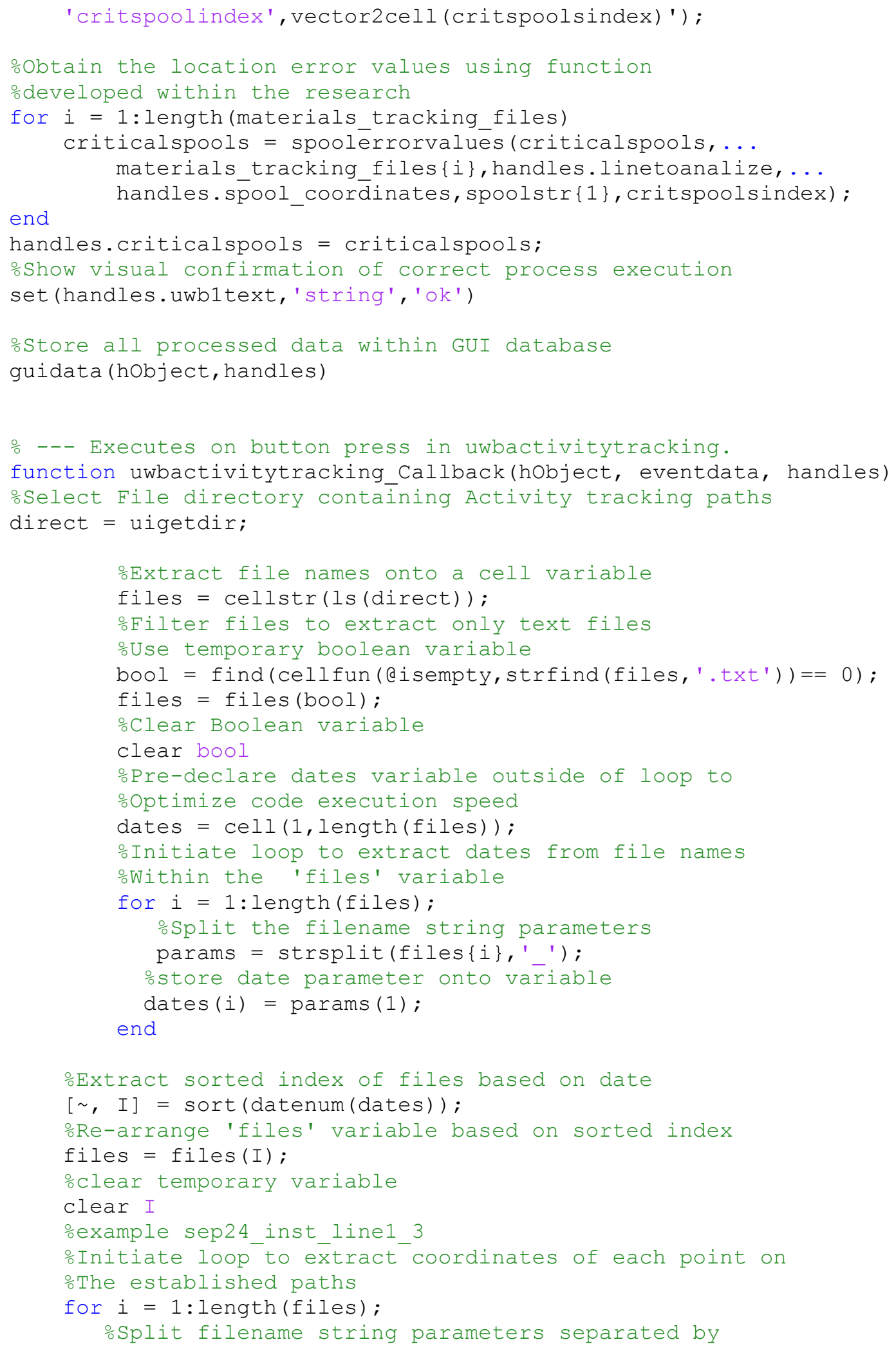




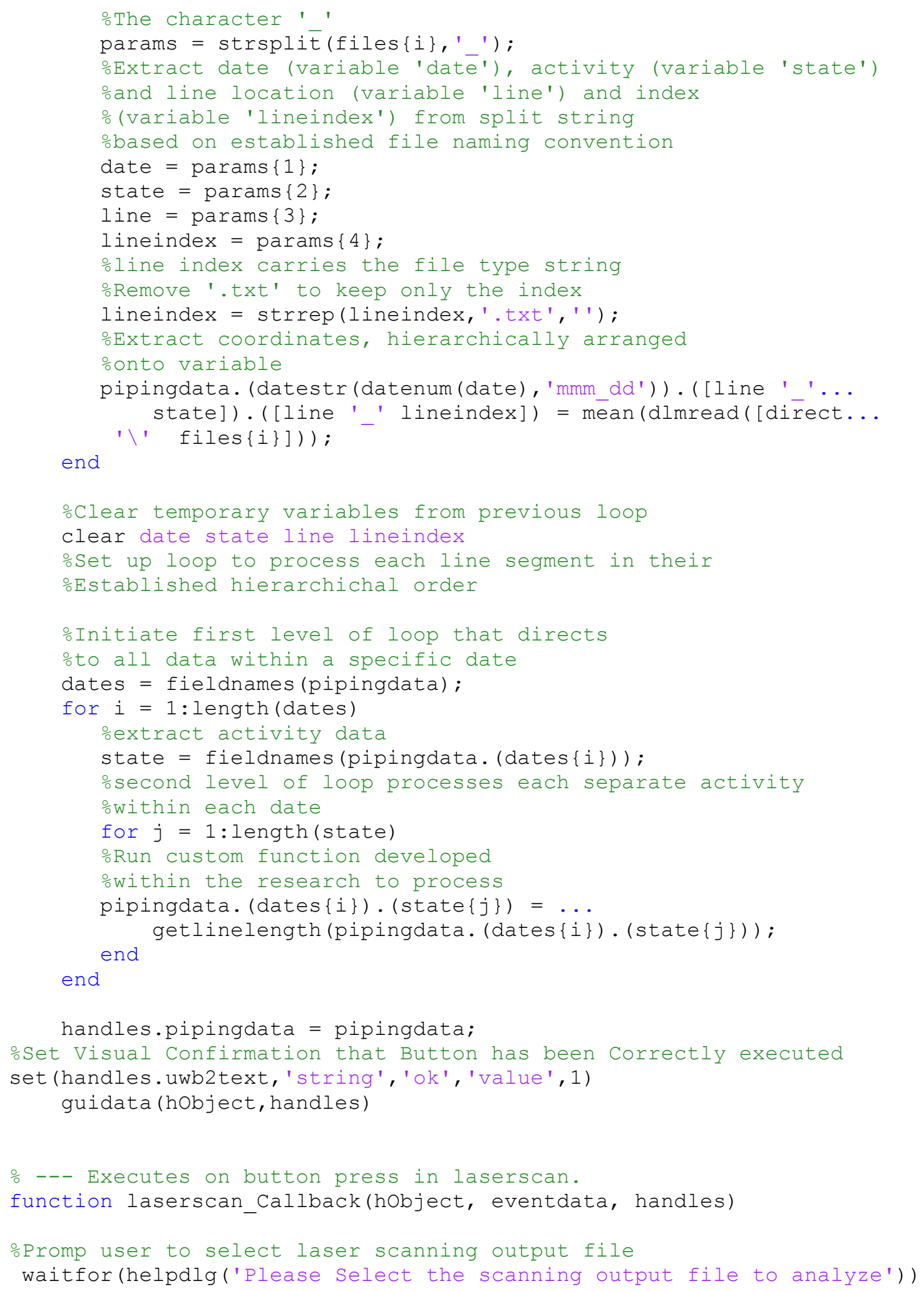




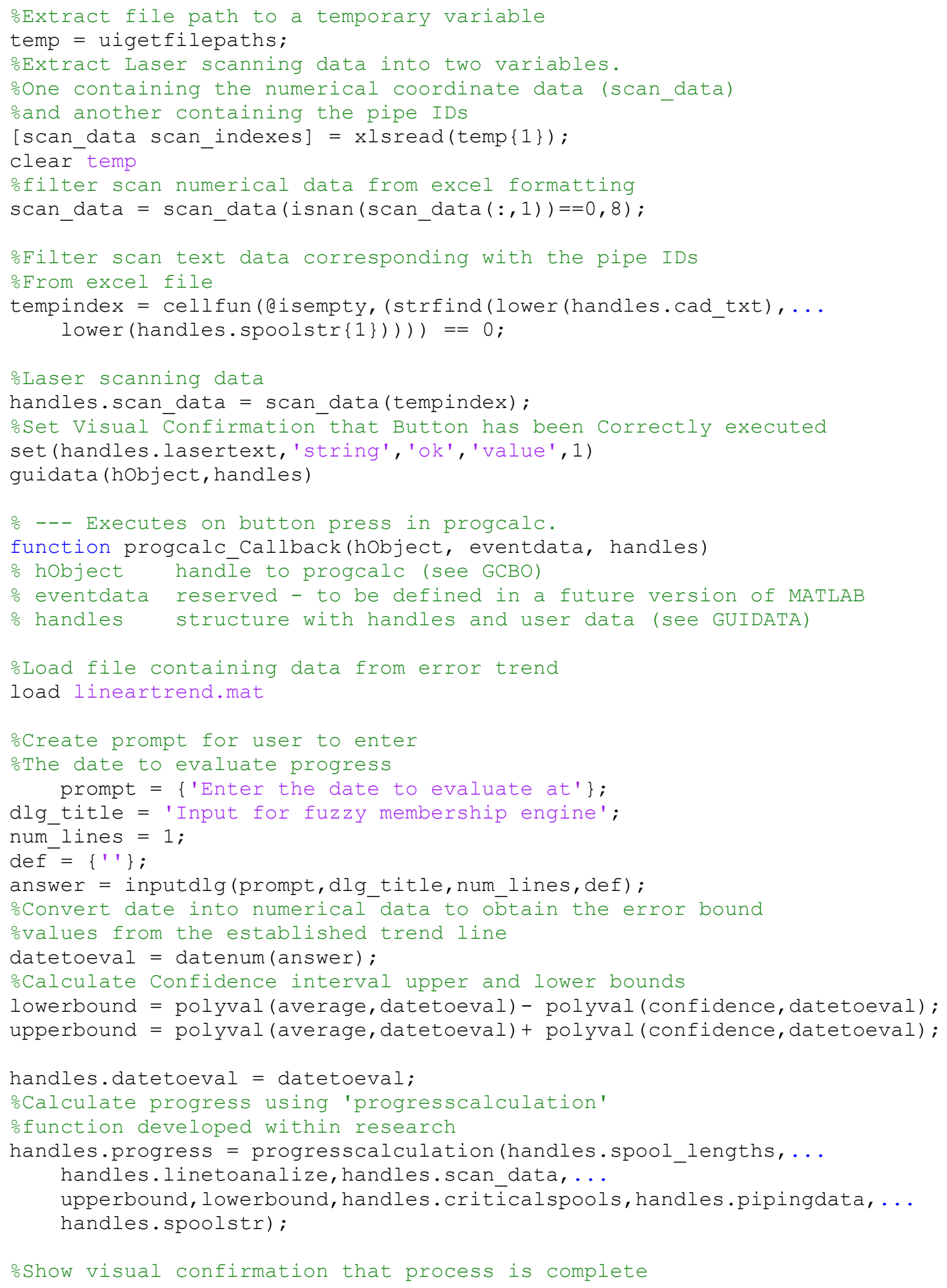




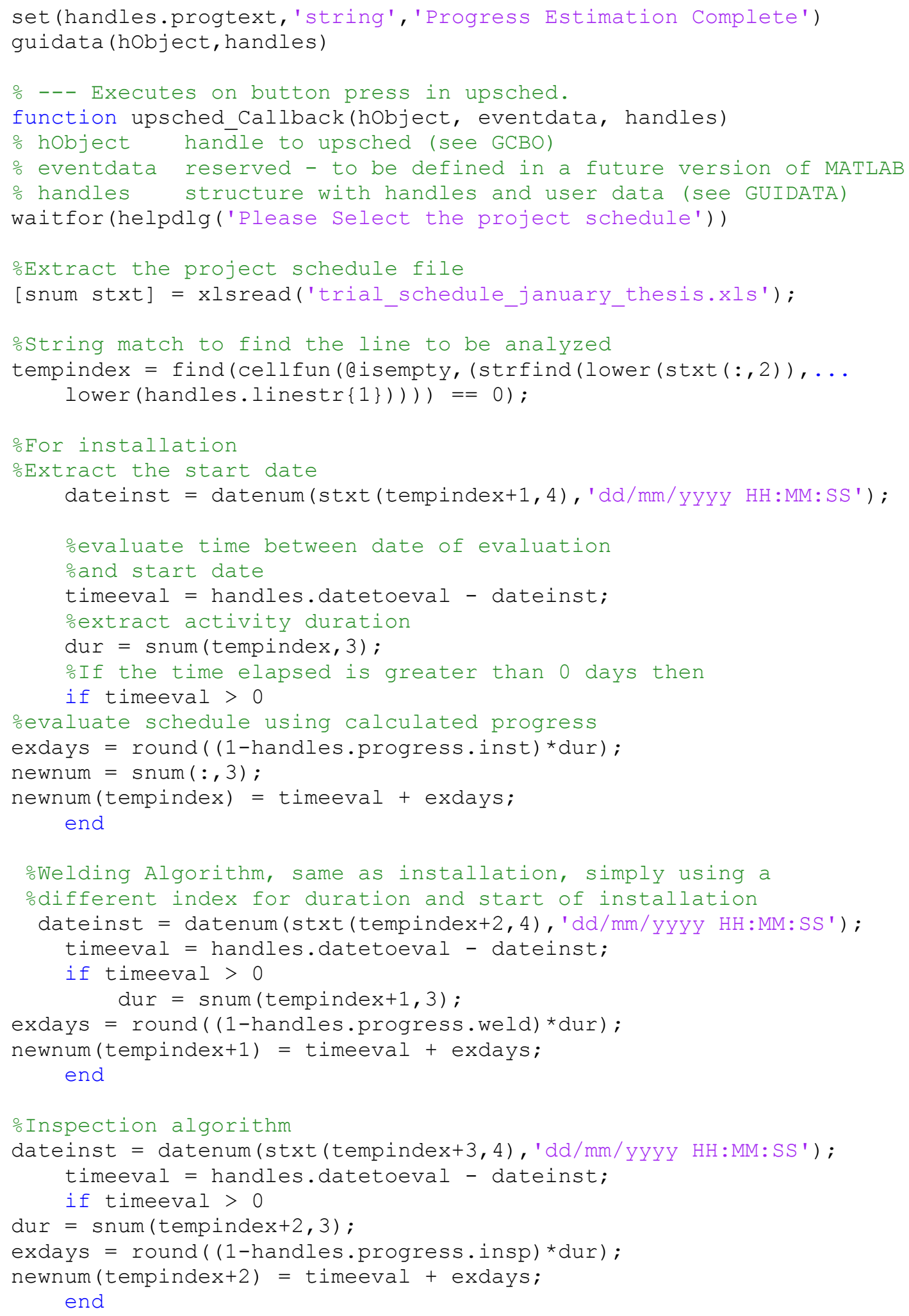




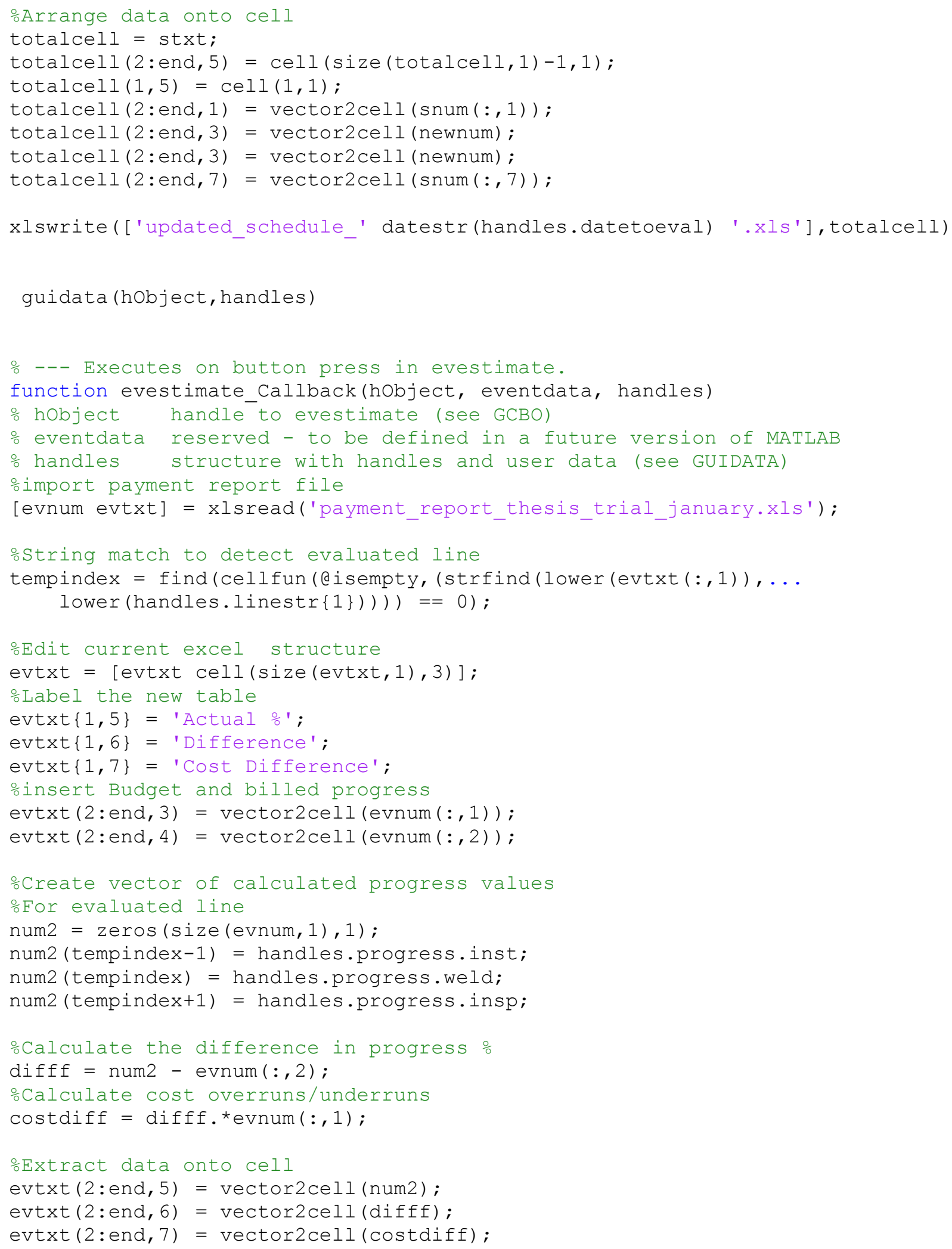




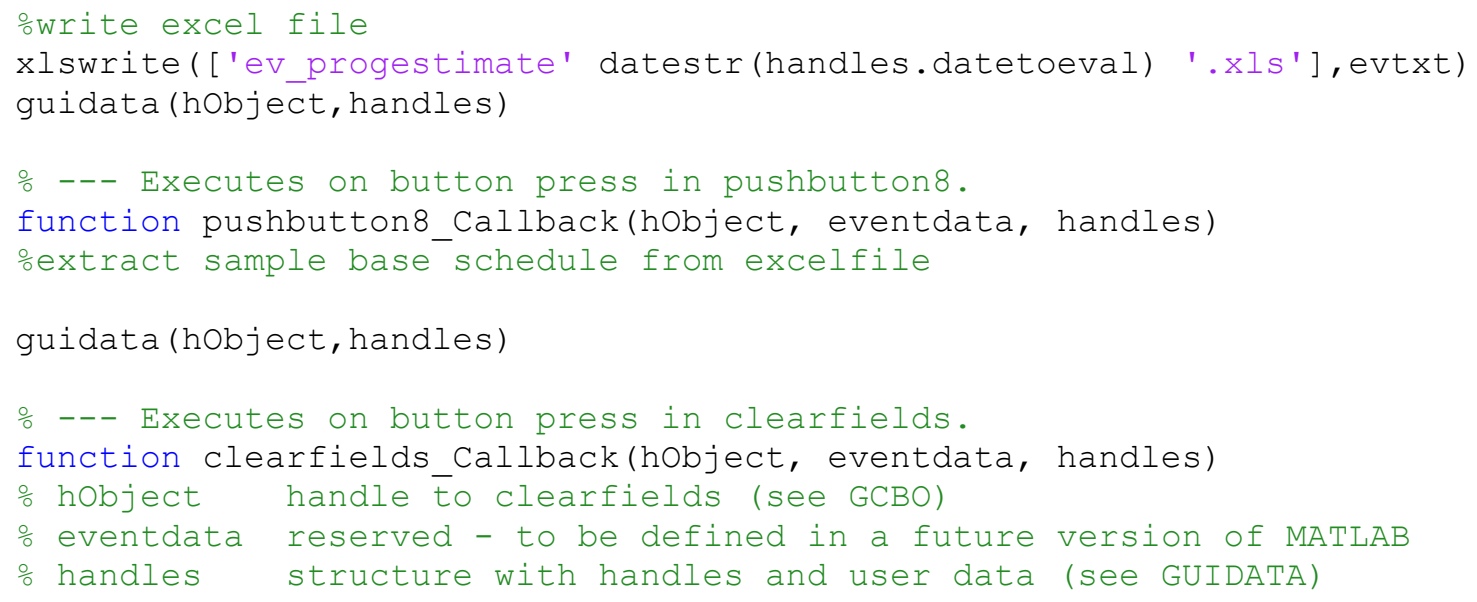

\section{B.2. Functions Developed for Data Fusion Processes}

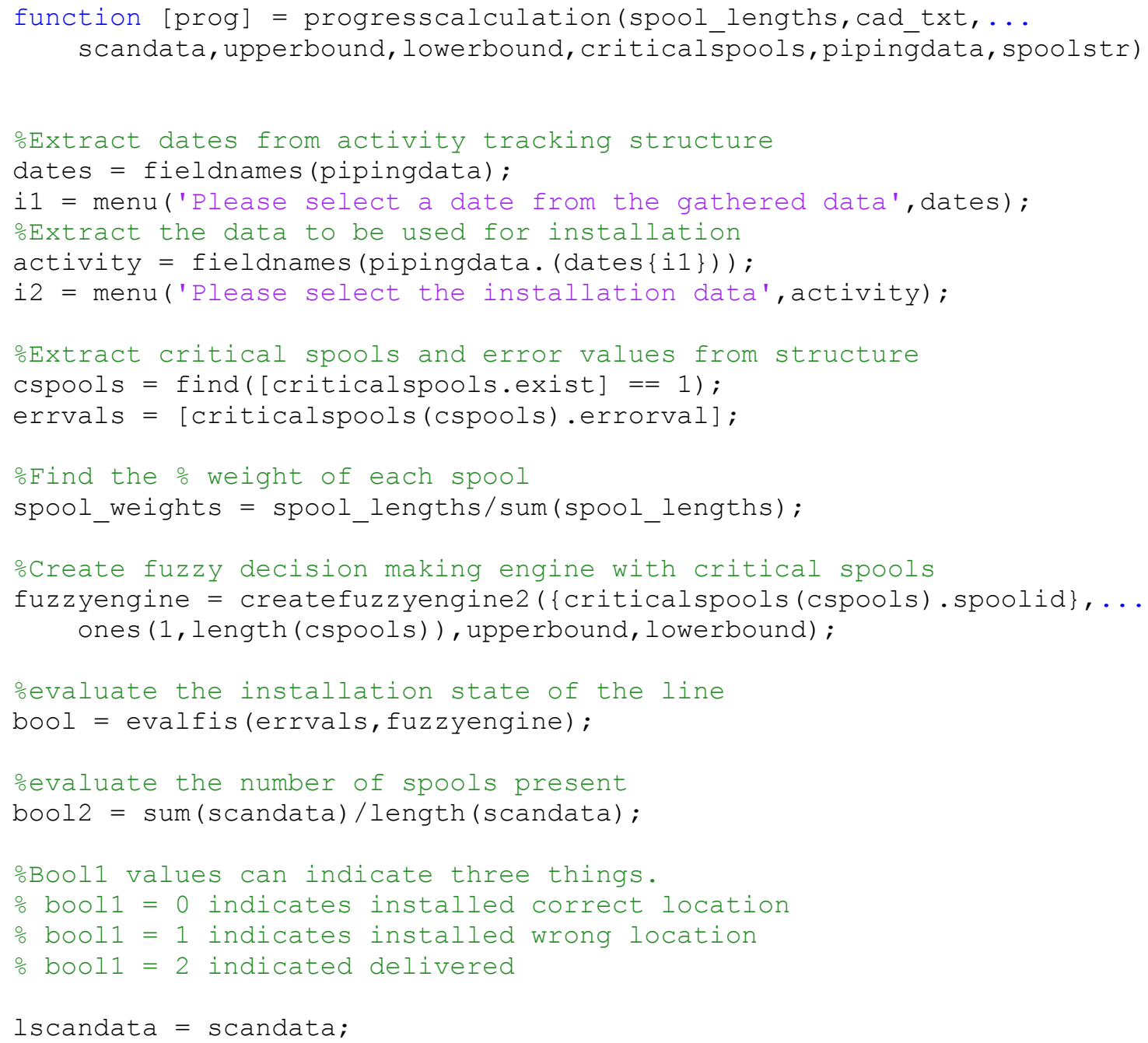




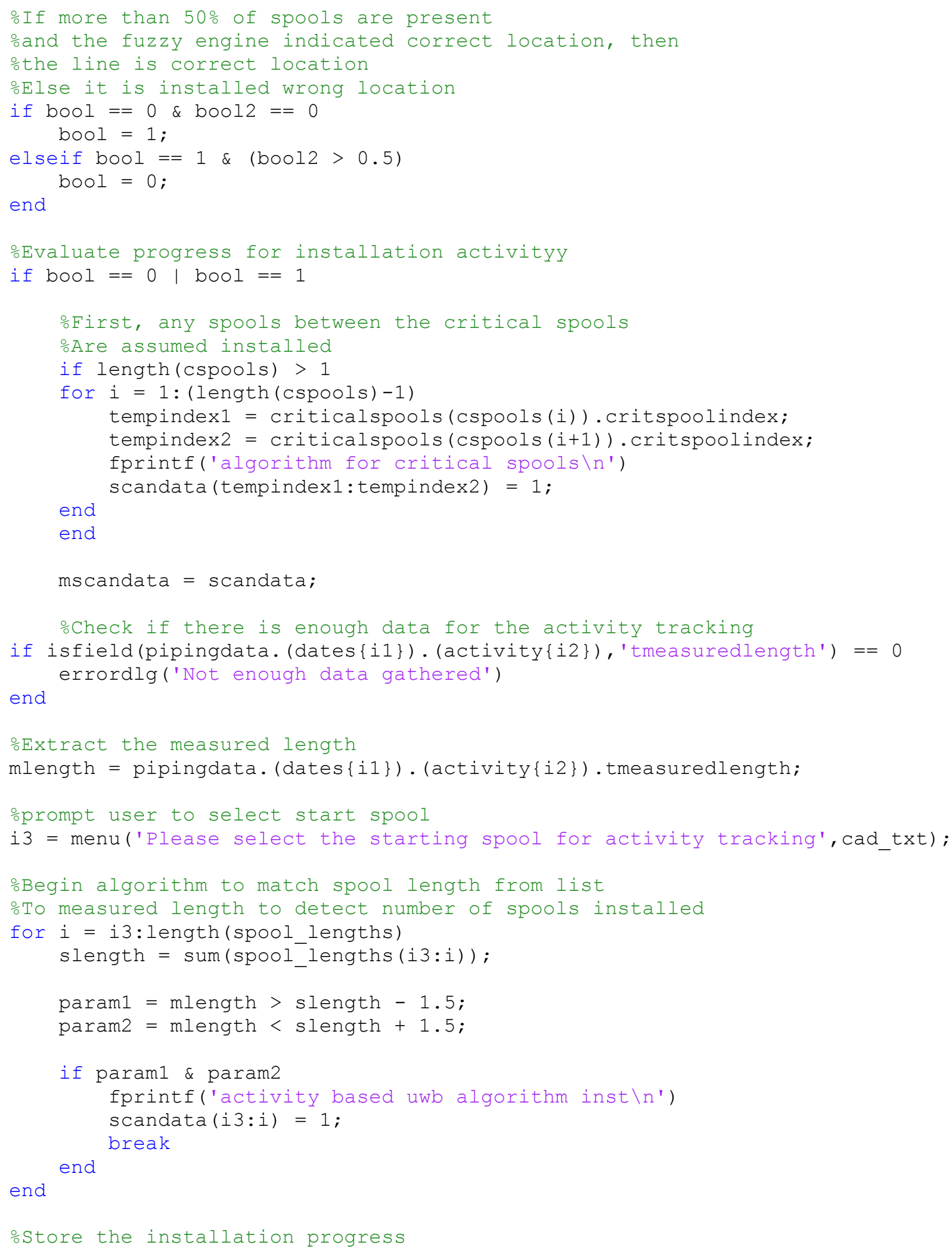




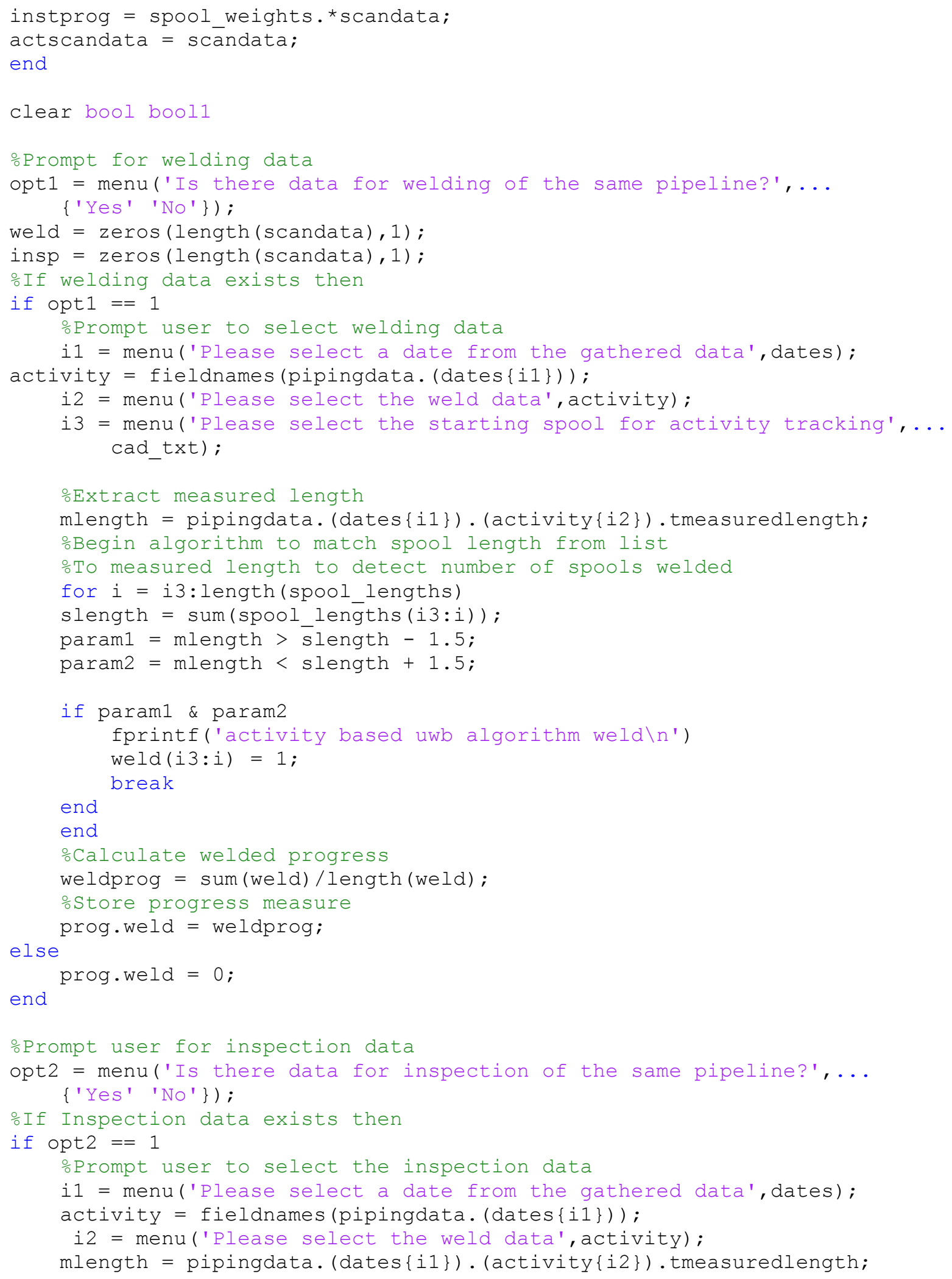




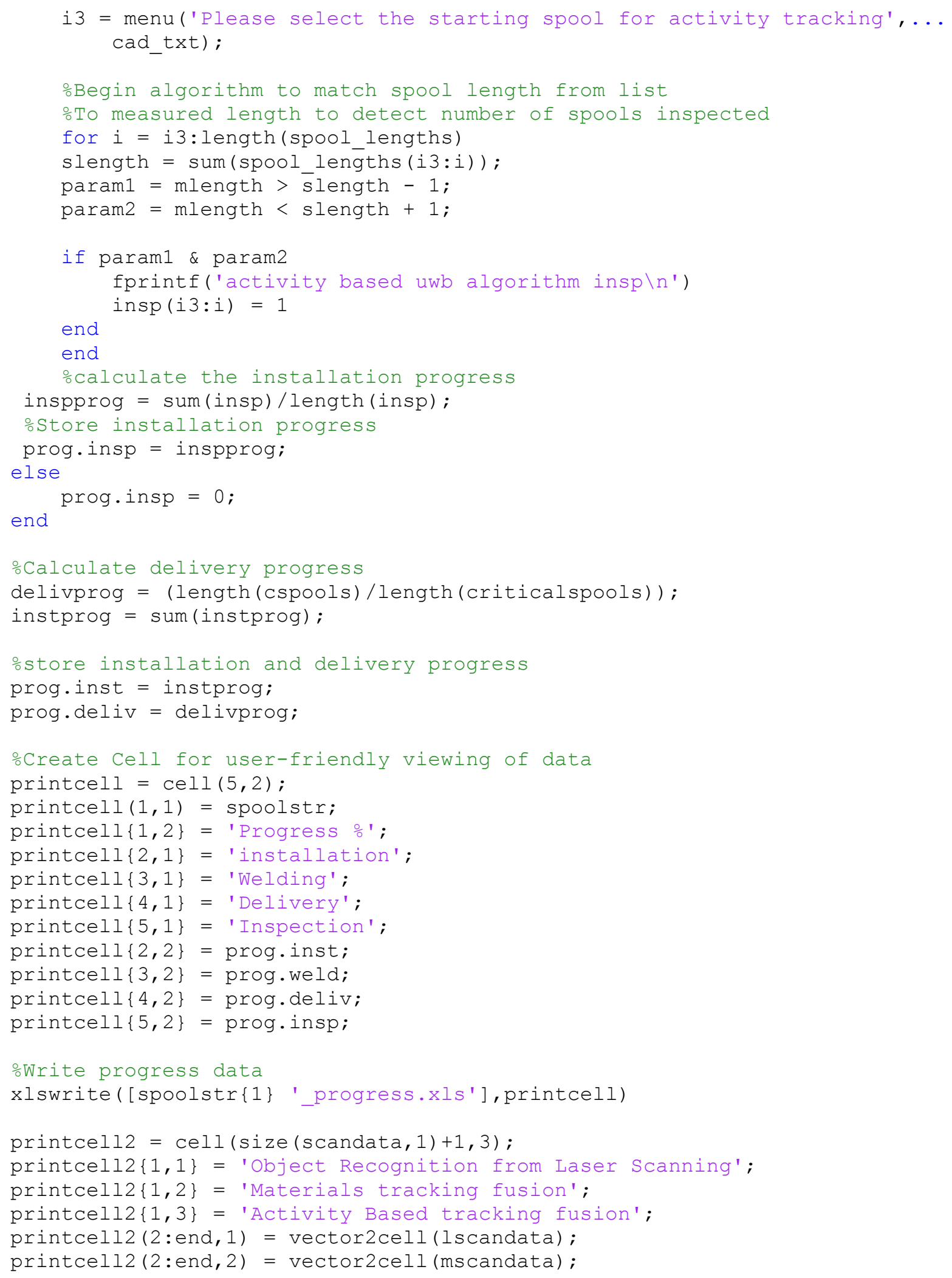




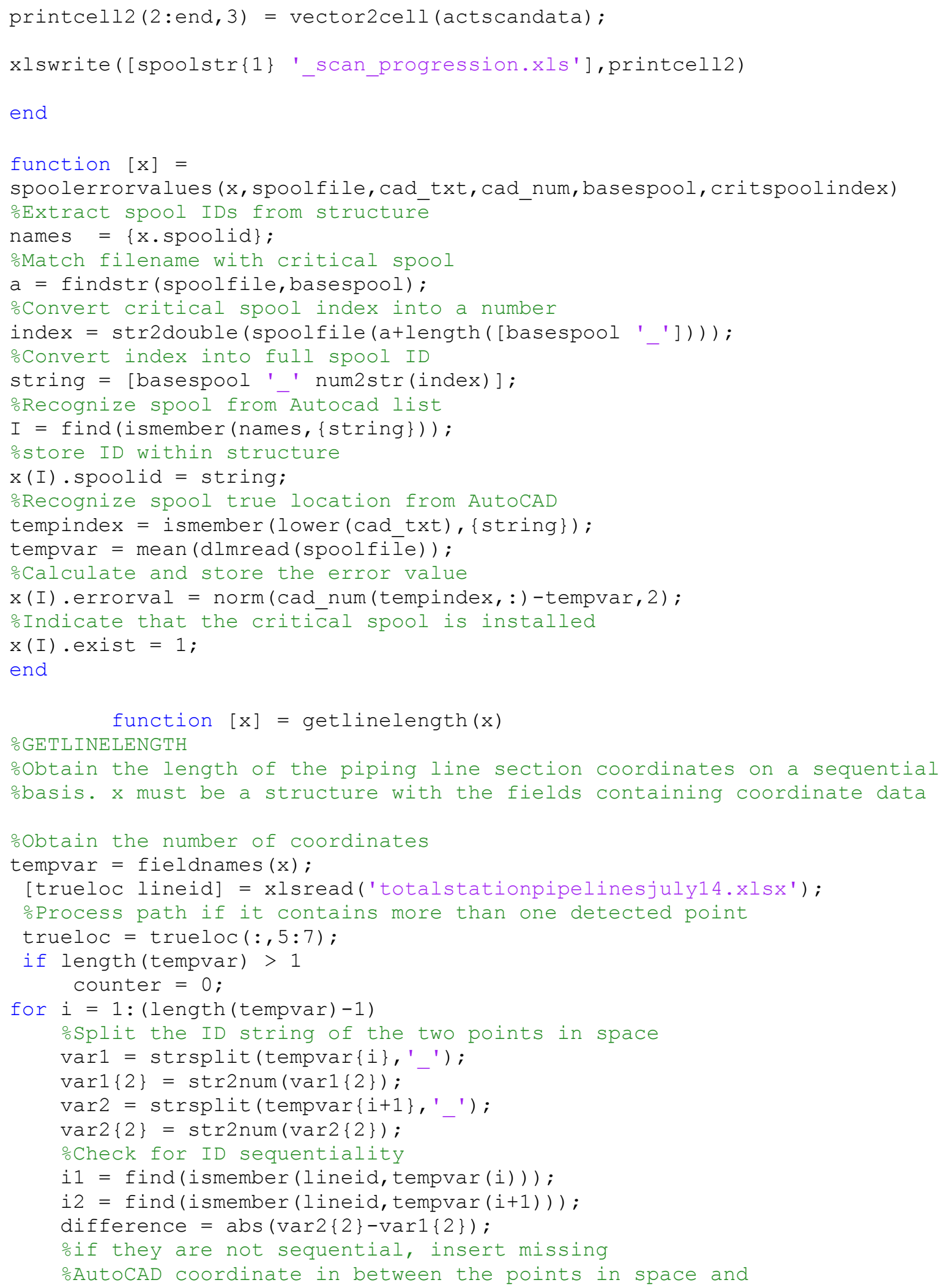




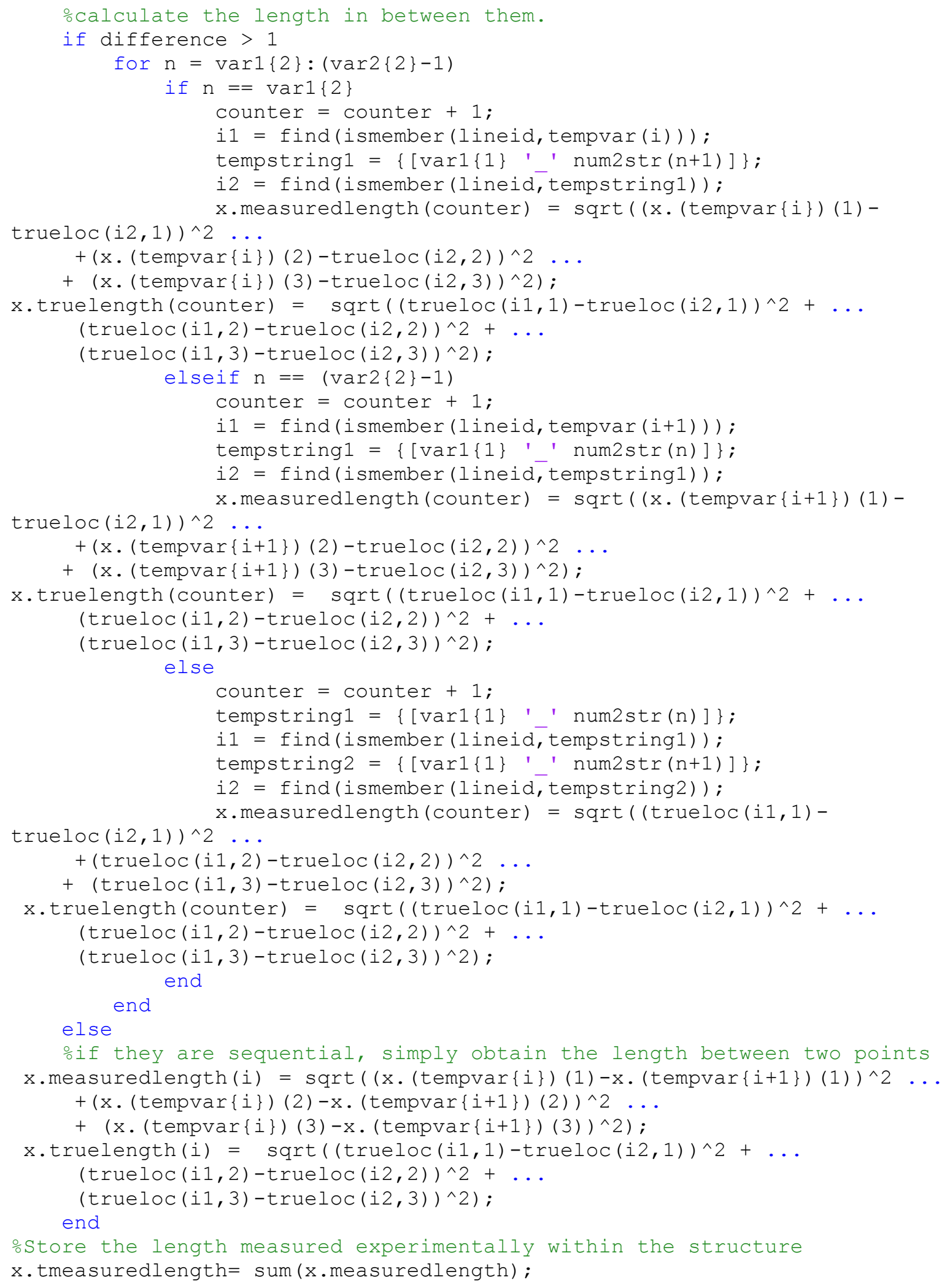




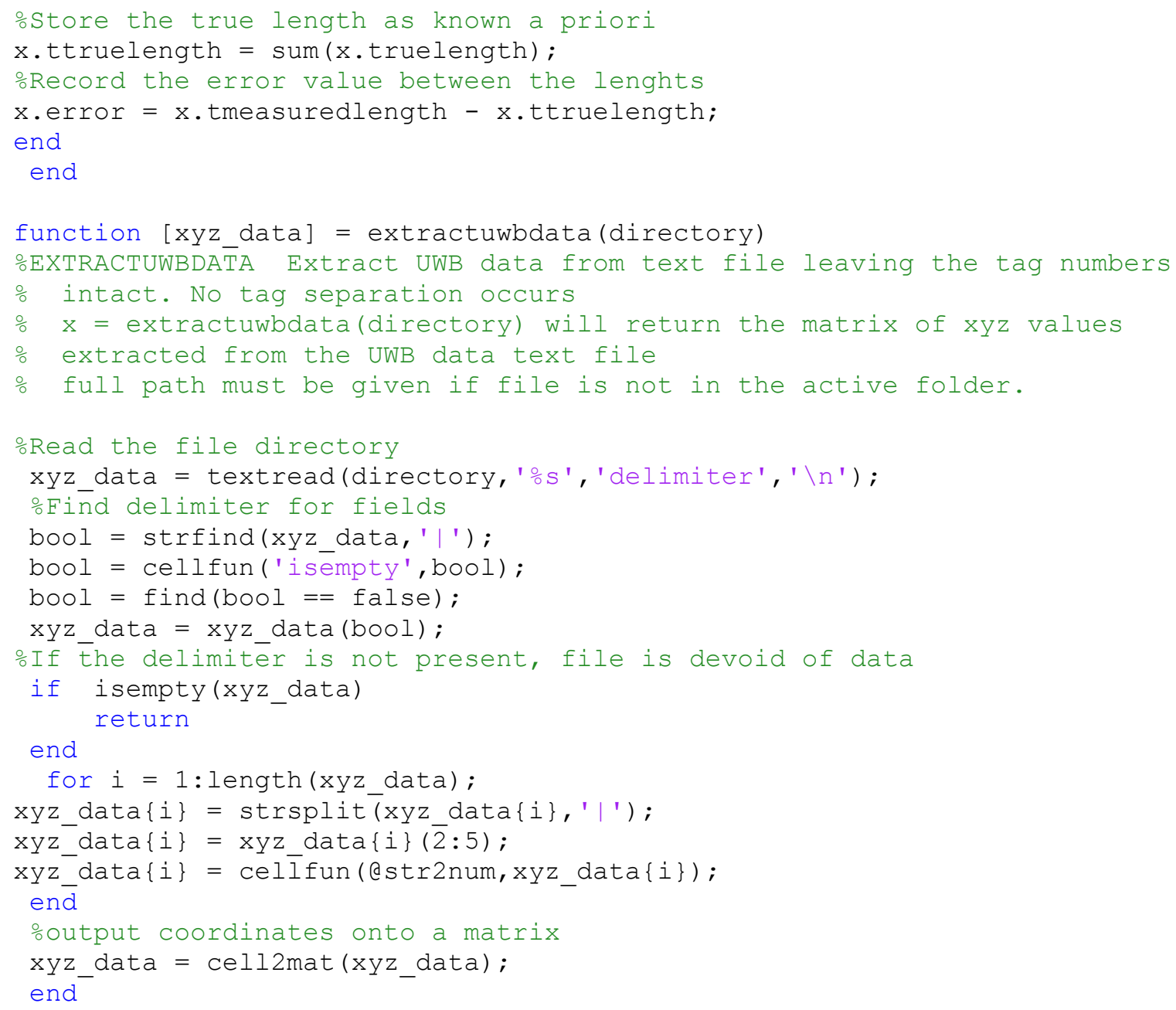




\section{Appendix C \\ Selected Data Files}

This appendix includes a brief summary of a limited and selected number of files, from the files used in the analysis of this thesis. During the laboratory and field experiments of this research, over $50 \mathrm{~GB}$ of data in various formats was collected and therefore including even a representative amount of data in this appendix was not feasible. The entire data collected for this research project is made available online through the EPPMS solution developed for construction research data management, as presented in Chapter 6 of this thesis. The following tables of this appendix are commented with descriptive captions to explain the context of data presented. Sample progress estimates are provides for one day, October $30^{\text {th }} 2010$, as explained in the captions.

Table C-1: Error Summary for a Selection of UWB Readings during Field Experiments on UW Engineering IV Building

\begin{tabular}{clccc}
\hline Tag ID & Date & Location & Error & $\begin{array}{c}\text { Error Standard } \\
\text { Deviation }\end{array}$ \\
\hline tag170 & Oct_06_2010 & cal8c & 0.198 & 0.037 \\
tag78 & Oct_06_2010 & cal8c & 0.300 & 0.055 \\
tag89 & Oct_06_2010 & cal8c & 0.418 & 0.039 \\
tag89 & Oct_13_2010 & cal8c & 0.333 & 0.029 \\
tag78 & Oct_13_2010 & cal8c & 0.362 & 0.173 \\
tag170 & Sep_21_2010 & col3l & 0.616 & 0.015 \\
tag78 & Sep_21_2010 & col3l & 0.631 & 0.008 \\
tag89 & Sep_21_2010 & col3l & 0.632 & 0.012 \\
tag89 & Sep_24_2010 & col3l & 0.099 & 0.006 \\
tag78 & Sep_24_2010 & col3l & 0.518 & 0.017 \\
tag170 & Sep_29_2010 & col4r & 0.386 & 0.032 \\
tag89 & Sep_29_2010 & col4r & 0.499 & 0.032 \\
tag78 & Nov_09_2010 & gr2 & 0.411 & 0.008 \\
tag170 & Nov_11_2010 & gr2 & 0.392 & 0.011 \\
tag174 & Nov_11_2010 & gr2 & 0.474 & 0.097 \\
tag78 & Oct_01_2010 & gr2 & 0.558 & 0.015 \\
tag89 & Oct_07_2010 & gr2 & 0.662 & 0.027 \\
tag89 & Oct_19_2010 & gr2 & 0.427 & 0.009 \\
tag78 & Sep_24_2010 & gr2 & 0.391 & 0.013 \\
tag89 & Sep_29_2010 & gr2 & 0.313 & 0.017 \\
\hline
\end{tabular}




\begin{tabular}{|c|c|c|c|c|}
\hline tag78 & Oct_01_2010 & gr3 & 0.100 & 0.004 \\
\hline tag170 & Oct_01_2010 & gr3 & 0.152 & 0.018 \\
\hline tag89 & Oct_01_2010 & gr3 & 0.417 & 1.719 \\
\hline tag89 & Oct_06_2010 & gr3 & 0.376 & 0.058 \\
\hline $\operatorname{tag} 78$ & Oct_06_2010 & gr3 & 0.409 & 0.104 \\
\hline tag170 & Oct_06_2010 & gr3 & 0.492 & 0.072 \\
\hline tag170 & Oct_07_2010 & gr3 & 0.505 & 0.088 \\
\hline $\operatorname{tag} 170$ & Oct_07_2010 & gr3 & 0.537 & 0.111 \\
\hline $\operatorname{tag} 78$ & Oct_13_2010 & gr3 & 0.439 & 0.096 \\
\hline tag89 & Oct_13_2010 & gr3 & 0.460 & 0.046 \\
\hline $\operatorname{tag} 170$ & Oct_13_2010 & gr3 & 0.476 & 0.089 \\
\hline $\operatorname{tag} 78$ & Sep_24_2010 & gr3 & 0.366 & 0.018 \\
\hline $\operatorname{tag} 78$ & Sep_28_2010 & gr3 & 0.277 & 0.015 \\
\hline $\operatorname{tag} 89$ & Sep_29_2010 & gr3 & 0.274 & 0.019 \\
\hline $\operatorname{tag} 89$ & Oct_06_2010 & gr4 & 0.517 & 0.124 \\
\hline tag170 & Oct_06_2010 & gr4 & 0.584 & 0.075 \\
\hline tag89 & Oct_13_2010 & gr4 & 0.372 & 0.115 \\
\hline tag170 & Oct_13_2010 & gr4 & 0.420 & 0.081 \\
\hline $\operatorname{tag} 78$ & Oct_13_2010 & gr4 & 0.538 & 0.121 \\
\hline tag174 & Nov_09_2010 & hr1 & 0.203 & 0.039 \\
\hline $\operatorname{tag} 174$ & Nov_09_2010 & hr1 & 0.204 & 0.043 \\
\hline tag170 & Oct_05_2010 & hr1 & 0.363 & 0.015 \\
\hline $\operatorname{tag} 78$ & Oct_07_2010 & hr1 & 0.383 & 0.015 \\
\hline $\operatorname{tag} 78$ & Oct_07_2010 & hr1 & 0.416 & 0.019 \\
\hline $\operatorname{tag} 78$ & Oct_19_2010 & hr1 & 0.248 & 0.026 \\
\hline tag89 & Nov_09_2010 & $\mathrm{hr} 2$ & 0.429 & 0.034 \\
\hline tag89 & Nov_09_2010 & $\mathrm{hr} 2$ & 0.446 & 0.024 \\
\hline tag170 & Nov_11_2010 & $\mathrm{hr} 2$ & 0.408 & 0.020 \\
\hline tag89 & Oct_07_2010 & $\mathrm{hr} 2$ & 0.349 & 0.038 \\
\hline tag89 & Oct_07_2010 & $\mathrm{hr} 2$ & 0.351 & 0.052 \\
\hline tag89 & Oct_19_2010 & $\mathrm{hr} 2$ & 0.353 & 0.026 \\
\hline tag170 & Oct_19_2010 & hr3 & 0.305 & 0.158 \\
\hline tag89 & Nov_11_2010 & $\mathrm{p} 11$ & 0.496 & 0.168 \\
\hline tag170 & Oct_05_2010 & $\mathrm{p} 11$ & 0.251 & 0.169 \\
\hline $\operatorname{tag} 78$ & Oct_07_2010 & $\mathrm{p} 11$ & 0.218 & 0.134 \\
\hline $\operatorname{tag} 78$ & Oct_07_2010 & $\mathrm{p} 11$ & 0.222 & 0.169 \\
\hline $\operatorname{tag} 78$ & Oct_19_2010 & $\mathrm{p} 11$ & 0.552 & 0.116 \\
\hline tag89 & Oct_07_2010 & $\mathrm{p} 12$ & 0.439 & 0.210 \\
\hline tag89 & Oct_07_2010 & $\mathrm{p} 12$ & 0.558 & 0.195 \\
\hline $\operatorname{tag} 78$ & Nov_11_2010 & $\mathrm{p} 13$ & 0.615 & 0.126 \\
\hline
\end{tabular}




\begin{tabular}{crrrl}
\hline tag170 & Oct_07_2010 & p13 & 0.325 & 0.063 \\
tag170 & Oct_07_2010 & p13 & 0.328 & 0.076 \\
tag89 & Nov_09_2010 & p21 & 0.474 & 0.286 \\
tag170 & Oct_05_2010 & p21 & 0.453 & 0.037 \\
tag78 & Oct_07_2010 & p21 & 0.344 & 0.065 \\
tag78 & Oct_07_2010 & p21 & 0.357 & 0.045 \\
tag78 & Oct_19_2010 & p21 & 0.510 & 0.224 \\
tag89 & Oct_19_2010 & p22 & 0.531 & 0.249 \\
tag170 & Oct_07_2010 & p31 & 0.445 & 0.074 \\
tag170 & Oct_07_2010 & p31 & 0.495 & 0.025 \\
\hline
\end{tabular}

Table C-2: One of over 240 UWB Files Used from Field Data Collection on Engineering IV Building

\begin{tabular}{ccccc}
\hline Tag ID & $\mathbf{X}$ & $\mathbf{Y}$ & $\mathbf{Z}$ & Time Stamp \\
\hline 20000098174 & 11.583 & 0.325 & -0.21 & $11 / 11 / 20105: 34: 03$ PM \\
20000098078 & 6.338 & -1.509 & 1.141 & $11 / 11 / 20105: 34: 03$ PM \\
20000098170 & -4.59 & -1.016 & 0.132 & $11 / 11 / 20105: 34: 03$ PM \\
20000098078 & 6.347 & -1.507 & 1.117 & $11 / 11 / 20105: 34: 04$ PM \\
20000100089 & 0.056 & -0.032 & 0.087 & $11 / 11 / 20105: 34: 04$ PM \\
20000098169 & -12.482 & -0.131 & 2.476 & $11 / 11 / 20105: 34: 04$ PM \\
20000098170 & -4.592 & -1.02 & 0.129 & $11 / 11 / 20105: 34: 04$ PM \\
20000098174 & 11.541 & 0.198 & -0.146 & $11 / 11 / 20105: 34: 04$ PM \\
20000098078 & 6.348 & -1.497 & 1.1 & $11 / 11 / 20105: 34: 04$ PM \\
20000098170 & -4.594 & -1.022 & 0.12 & $11 / 11 / 20105: 34: 04$ PM \\
20000098078 & 6.349 & -1.493 & 1.069 & $11 / 11 / 20105: 34: 05$ PM \\
20000100089 & 0.058 & -0.03 & 0.079 & $11 / 11 / 20105: 34: 05$ PM \\
20000098169 & -6.876 & -0.715 & 1.288 & $11 / 11 / 20105: 34: 05$ PM \\
20000098170 & -4.589 & -1.021 & 0.122 & $11 / 11 / 20105: 34: 05$ PM \\
20000098174 & 11.574 & 0.28 & -0.2 & $11 / 11 / 20105: 34: 05$ PM \\
20000098078 & 6.353 & -1.485 & 1.044 & $11 / 11 / 20105: 34: 05$ PM \\
20000100089 & 0.054 & -0.03 & 0.077 & $11 / 11 / 20105: 34: 05$ PM \\
20000098170 & -4.59 & -1.024 & 0.117 & $11 / 11 / 20105: 34: 05$ PM \\
20000098078 & 6.353 & -1.477 & 1.016 & $11 / 11 / 20105: 34: 05$ PM \\
20000100089 & 0.051 & -0.031 & 0.075 & $11 / 11 / 20105: 34: 05$ PM \\
20000098169 & -5.896 & -0.664 & 1.575 & $11 / 11 / 20105: 34: 06$ PM \\
20000098170 & -4.597 & -1.021 & 0.131 & $11 / 11 / 20105: 34: 06$ PM \\
20000098174 & 11.557 & 0.349 & -0.148 & $11 / 11 / 20105: 34: 06$ PM \\
20000098078 & 6.357 & -1.48 & 0.984 & $11 / 11 / 20105: 34: 06$ PM \\
20000100089 & 0.039 & -0.031 & 0.07 & $11 / 11 / 20105: 34: 06$ PM \\
20000098170 & -4.595 & -1.019 & 0.135 & $11 / 11 / 20105: 34: 06$ PM \\
20000098078 & 6.355 & -1.487 & 0.993 & $11 / 11 / 20105: 34: 06$ PM \\
20000100089 & 0.04 & -0.03 & 0.064 & $11 / 11 / 20105: 34: 06$ PM \\
\hline
\end{tabular}




\begin{tabular}{|c|c|c|c|c|}
\hline 20000098169 & -6.714 & -0.666 & 1.21 & 11/11/2010 5:34:06 PM \\
\hline 20000098170 & -4.599 & -1.014 & 0.145 & 11/11/2010 5:34:07 PM \\
\hline 20000098174 & 11.586 & 0.355 & -0.171 & 11/11/2010 5:34:07 PM \\
\hline 20000098078 & 6.354 & -1.492 & 0.991 & 11/11/2010 5:34:07 PM \\
\hline 20000098170 & -4.599 & -1.011 & 0.146 & 11/11/2010 5:34:07 PM \\
\hline 20000098078 & 6.353 & -1.487 & 0.977 & 11/11/2010 5:34:07 PM \\
\hline 20000100089 & 0.035 & -0.033 & 0.067 & 11/11/2010 5:34:07 PM \\
\hline 20000098169 & -5.731 & -0.704 & 1.578 & 11/11/2010 5:34:07 PM \\
\hline 20000098170 & -4.592 & -1.012 & 0.143 & 11/11/2010 5:34:07 PM \\
\hline 20000098174 & 11.563 & 0.299 & -0.152 & 11/11/2010 5:34:07 PM \\
\hline 20000098078 & 6.318 & -1.455 & 1.402 & 11/11/2010 5:34:08 PM \\
\hline 20000100089 & 0.034 & -0.036 & 0.066 & 11/11/2010 5:34:08 PM \\
\hline 20000098170 & -4.586 & -1.011 & 0.136 & 11/11/2010 5:34:08 PM \\
\hline 20000098078 & 6.323 & -1.45 & 1.376 & 11/11/2010 5:34:08 PM \\
\hline 20000100089 & 0.037 & -0.037 & 0.063 & 11/11/2010 5:34:08 PM \\
\hline 20000098169 & -6.088 & -0.974 & 1.198 & 11/11/2010 5:34:08 PM \\
\hline 20000098170 & -4.587 & -1.006 & 0.129 & 11/11/2010 5:34:08 PM \\
\hline 20000098174 & 11.605 & 0.401 & -0.198 & 11/11/2010 5:34:08 PM \\
\hline 20000098078 & 6.326 & -1.442 & 1.374 & 11/11/2010 5:34:08 PM \\
\hline 20000100089 & 0.036 & -0.033 & 0.057 & 11/11/2010 5:34:08 PM \\
\hline 20000098170 & -4.584 & -1.001 & 0.12 & 11/11/2010 5:34:09 PM \\
\hline 20000098078 & 6.326 & -1.44 & 1.352 & 11/11/2010 5:34:09 PM \\
\hline 20000098169 & -12.466 & 0.11 & 2.643 & $11 / 11 / 2010$ 5:34:09 PM \\
\hline 20000098170 & -4.593 & -0.997 & 0.122 & 11/11/2010 5:34:09 PM \\
\hline 20000098174 & 11.675 & 0.204 & -0.44 & 11/11/2010 5:34:09 PM \\
\hline 20000098078 & 6.329 & -1.436 & 1.321 & 11/11/2010 5:34:09 PM \\
\hline 20000098170 & -4.597 & -1.001 & 0.128 & 11/11/2010 5:34:10 PM \\
\hline 20000098078 & 6.334 & -1.436 & 1.286 & 11/11/2010 5:34:10 PM \\
\hline 20000100089 & 0.039 & -0.033 & 0.068 & 11/11/2010 5:34:10 PM \\
\hline 20000098169 & -12.506 & 0.074 & 2.391 & $11 / 11 / 2010$ 5:34:10 PM \\
\hline 20000098170 & -4.598 & -1.005 & 0.127 & 11/11/2010 5:34:10 PM \\
\hline 20000098174 & 11.593 & 0.335 & -0.245 & 11/11/2010 5:34:10 PM \\
\hline 20000098078 & 6.337 & -1.438 & 1.246 & 11/11/2010 5:34:10 PM \\
\hline 20000100089 & 0.037 & -0.033 & 0.074 & 11/11/2010 5:34:10 PM \\
\hline 20000098170 & -4.598 & -1.006 & 0.118 & 11/11/2010 5:34:10 PM \\
\hline 20000098078 & 6.339 & -1.439 & 1.199 & 11/11/2010 5:34:11 PM \\
\hline 20000100089 & 0.041 & -0.034 & 0.075 & 11/11/2010 5:34:11 PM \\
\hline 20000098169 & -6.426 & -0.886 & 1.165 & $11 / 11 / 2010$ 5:34:11 PM \\
\hline 20000098170 & -4.596 & -1.008 & 0.106 & 11/11/2010 5:34:11 PM \\
\hline 20000098174 & 11.684 & 0.221 & -0.451 & 11/11/2010 5:34:11 PM \\
\hline 20000098078 & 6.341 & -1.44 & 1.168 & 11/11/2010 5:34:11 PM \\
\hline 20000100089 & 0.043 & -0.032 & 0.071 & $11 / 11 / 2010$ 5:34:11 PM \\
\hline 20000098170 & -4.592 & -1.012 & 0.102 & 11/11/2010 5:34:11 PM \\
\hline 20000098078 & 6.342 & -1.448 & 1.135 & 11/11/2010 5:34:11 PM \\
\hline 20000100089 & 0.051 & -0.031 & 0.083 & $11 / 11 / 2010$ 5:34:11 PM \\
\hline 20000098169 & -6.895 & -0.752 & 1.183 & 11/11/2010 5:34:12 PM \\
\hline 20000098170 & -4.58 & -1.012 & 0.103 & 11/11/2010 5:34:12 PM \\
\hline
\end{tabular}




\begin{tabular}{|c|c|c|c|c|}
\hline 20000098078 & 6.34 & -1.448 & 1.121 & 11/11/2010 5:34:12 PM \\
\hline 20000100089 & 0.048 & -0.033 & 0.093 & 11/11/2010 5:34:12 PM \\
\hline 20000098170 & -4.572 & -1.01 & 0.107 & 11/11/2010 5:34:12 PM \\
\hline 20000098078 & 6.342 & -1.452 & 1.098 & 11/11/2010 5:34:12 PM \\
\hline 20000100089 & 0.053 & -0.036 & 0.122 & 11/11/2010 5:34:12 PM \\
\hline 20000098169 & -6.815 & -0.86 & 1.242 & 11/11/2010 5:34:12 PM \\
\hline 20000098170 & -4.567 & -1.01 & 0.108 & 11/11/2010 5:34:13 PM \\
\hline 20000098174 & 11.597 & 0.342 & -0.243 & 11/11/2010 5:34:13 PM \\
\hline 20000098078 & 6.338 & -1.438 & 1.102 & 11/11/2010 5:34:13 PM \\
\hline 20000100089 & 0.057 & -0.038 & 0.13 & 11/11/2010 5:34:13 PM \\
\hline 20000098170 & -4.57 & -1.018 & 0.102 & 11/11/2010 5:34:13 PM \\
\hline 20000098078 & 6.333 & -1.431 & 1.112 & 11/11/2010 5:34:13 PM \\
\hline 20000098169 & -12.494 & -0.103 & 2.436 & 11/11/2010 5:34:13 PM \\
\hline 20000098174 & 11.543 & 0.255 & -0.138 & 11/11/2010 5:34:14 PM \\
\hline 20000098078 & 6.332 & -1.423 & 1.129 & 11/11/2010 5:34:14 PM \\
\hline 20000100089 & 0.062 & -0.036 & 0.131 & $11 / 11 / 2010$ 5:34:14 PM \\
\hline 20000098078 & 6.328 & -1.411 & 1.13 & 11/11/2010 5:34:14 PM \\
\hline 20000100089 & 0.059 & -0.035 & 0.128 & 11/11/2010 5:34:14 PM \\
\hline 20000098169 & -5.153 & -1.032 & 1.402 & 11/11/2010 5:34:14 PM \\
\hline 20000098174 & 11.591 & 0.342 & -0.168 & 11/11/2010 5:34:14 PM \\
\hline 20000098078 & 6.332 & -1.408 & 1.119 & 11/11/2010 5:34:14 PM \\
\hline 20000100089 & 0.054 & -0.036 & 0.128 & 11/11/2010 5:34:15 PM \\
\hline 20000098078 & 6.334 & -1.398 & 1.105 & 11/11/2010 5:34:15 PM \\
\hline 20000100089 & 0.055 & -0.035 & 0.122 & 11/11/2010 5:34:15 PM \\
\hline 20000098169 & -6.182 & -0.997 & 1.237 & 11/11/2010 5:34:15 PM \\
\hline 20000098170 & -4.577 & -1.007 & 0.104 & 11/11/2010 5:34:15 PM \\
\hline 20000098174 & 11.796 & 0.553 & -0.753 & 11/11/2010 5:34:15 PM \\
\hline 20000098078 & 6.339 & -1.399 & 1.086 & 11/11/2010 5:34:15 PM \\
\hline 20000100089 & 0.053 & -0.033 & 0.117 & 11/11/2010 5:34:15 PM \\
\hline 20000098170 & -4.578 & -1.002 & 0.101 & 11/11/2010 5:34:16 PM \\
\hline 20000098078 & 6.337 & -1.399 & 1.102 & 11/11/2010 5:34:16 PM \\
\hline 20000100089 & 0.048 & -0.033 & 0.14 & 11/11/2010 5:34:16 PM \\
\hline 20000098169 & -6.751 & -0.856 & 1.279 & 11/11/2010 5:34:16 PM \\
\hline 20000098170 & -4.58 & -1.003 & 0.101 & 11/11/2010 5:34:16 PM \\
\hline 20000098174 & 11.587 & 0.386 & -0.223 & 11/11/2010 5:34:16 PM \\
\hline 20000098078 & 6.333 & -1.403 & 1.123 & 11/11/2010 5:34:16 PM \\
\hline 20000100089 & 0.048 & -0.033 & 0.149 & 11/11/2010 5:34:16 PM \\
\hline 20000098170 & -4.581 & -1.003 & 0.097 & $11 / 11 / 2010$ 5:34:16 PM \\
\hline 20000098078 & 6.337 & -1.412 & 1.127 & 11/11/2010 5:34:17 PM \\
\hline 20000100089 & 0.05 & -0.035 & 0.145 & 11/11/2010 5:34:17 PM \\
\hline 20000098169 & -5.936 & -0.85 & 1.206 & 11/11/2010 5:34:17 PM \\
\hline 20000098170 & -4.582 & -1.005 & 0.109 & 11/11/2010 5:34:17 PM \\
\hline 20000098174 & 11.575 & 0.306 & -0.206 & 11/11/2010 5:34:17 PM \\
\hline 20000098078 & 6.331 & -1.413 & 1.13 & 11/11/2010 5:34:17 PM \\
\hline 20000100089 & 0.052 & -0.033 & 0.141 & $11 / 11 / 2010$ 5:34:17 PM \\
\hline 20000098170 & -4.576 & -1.006 & 0.111 & 11/11/2010 5:34:17 PM \\
\hline 20000098078 & 6.332 & -1.413 & 1.153 & 11/11/2010 5:34:17 PM \\
\hline
\end{tabular}




\begin{tabular}{|c|c|c|c|c|}
\hline 20000100089 & 0.049 & -0.032 & 0.142 & 11/11/2010 5:34:18 PM \\
\hline 20000098169 & -12.46 & -0.01 & 2.549 & 11/11/2010 5:34:18 PM \\
\hline 20000098170 & -4.577 & -1.007 & 0.105 & 11/11/2010 5:34:18 PM \\
\hline 20000098174 & 11.573 & 0.358 & -0.101 & 11/11/2010 5:34:18 PM \\
\hline 20000098078 & 6.33 & -1.412 & 1.162 & 11/11/2010 5:34:18 PM \\
\hline 20000100089 & 0.053 & -0.033 & 0.134 & 11/11/2010 5:34:18 PM \\
\hline 20000098170 & -4.582 & -1.006 & 0.097 & 11/11/2010 5:34:18 PM \\
\hline 20000098078 & 6.333 & -1.419 & 1.152 & 11/11/2010 5:34:18 PM \\
\hline 20000100089 & 0.048 & -0.03 & 0.123 & 11/11/2010 5:34:18 PM \\
\hline 20000098169 & -12.482 & 0.014 & 2.532 & 11/11/2010 5:34:19 PM \\
\hline 20000098170 & -4.59 & -1.011 & 0.107 & 11/11/2010 5:34:19 PM \\
\hline 20000098174 & 11.587 & 0.318 & -0.249 & 11/11/2010 5:34:19 PM \\
\hline 20000098078 & 6.333 & -1.41 & 1.152 & 11/11/2010 5:34:19 PM \\
\hline 20000100089 & 0.045 & -0.032 & 0.133 & 11/11/2010 5:34:19 PM \\
\hline 20000098170 & -4.595 & -1.015 & 0.124 & 11/11/2010 5:34:19 PM \\
\hline 20000098078 & 6.335 & -1.421 & 1.151 & $11 / 11 / 2010$ 5:34:19 PM \\
\hline 20000100089 & 0.054 & -0.035 & 0.13 & 11/11/2010 5:34:19 PM \\
\hline 20000098169 & -12.478 & 0.036 & 2.472 & 11/11/2010 5:34:19 PM \\
\hline 20000098170 & -4.592 & -1.013 & 0.13 & 11/11/2010 5:34:19 PM \\
\hline 20000098174 & 11.557 & 0.23 & -0.2 & $11 / 11 / 2010$ 5:34:20 PM \\
\hline 20000098078 & 6.334 & -1.427 & 1.142 & 11/11/2010 5:34:20 PM \\
\hline 20000100089 & 0.057 & -0.035 & 0.132 & 11/11/2010 5:34:20 PM \\
\hline 20000098170 & -4.584 & -1.011 & 0.131 & $11 / 11 / 2010$ 5:34:20 PM \\
\hline 20000098078 & 6.328 & -1.422 & 1.119 & 11/11/2010 5:34:20 PM \\
\hline 20000100089 & 0.057 & -0.037 & 0.134 & $11 / 11 / 2010$ 5:34:20 PM \\
\hline 20000098169 & -6.848 & -0.788 & 1.281 & 11/11/2010 5:34:20 PM \\
\hline 20000098170 & -4.584 & -1.01 & 0.127 & 11/11/2010 5:34:20 PM \\
\hline 20000098174 & 11.608 & 0.384 & -0.297 & $11 / 11 / 2010$ 5:34:20 PM \\
\hline 20000098078 & 6.332 & -1.424 & 1.093 & 11/11/2010 5:34:21 PM \\
\hline 20000100089 & 0.056 & -0.039 & 0.148 & 11/11/2010 5:34:21 PM \\
\hline 20000098170 & -4.591 & -1.013 & 0.117 & 11/11/2010 5:34:21 PM \\
\hline 20000098078 & 6.333 & -1.439 & 1.094 & 11/11/2010 5:34:21 PM \\
\hline 20000100089 & 0.059 & -0.039 & 0.172 & 11/11/2010 5:34:21 PM \\
\hline 20000098169 & -5.357 & -0.733 & 1.379 & 11/11/2010 5:34:21 PM \\
\hline 20000098170 & -4.6 & -1.016 & 0.123 & 11/11/2010 5:34:21 PM \\
\hline 20000098078 & 6.332 & -1.429 & 1.118 & 11/11/2010 5:34:21 PM \\
\hline 20000100089 & 0.055 & -0.039 & 0.178 & 11/11/2010 5:34:21 PM \\
\hline 20000098170 & -4.602 & -1.021 & 0.12 & $11 / 11 / 2010$ 5:34:22 PM \\
\hline 20000098078 & 6.329 & -1.512 & 1.624 & $11 / 11 / 2010$ 5:34:22 PM \\
\hline 20000100089 & 0.061 & -0.04 & 0.178 & 11/11/2010 5:34:22 PM \\
\hline 20000098169 & -5.574 & -0.761 & 1.428 & $11 / 11 / 2010$ 5:34:22 PM \\
\hline 20000098170 & -4.599 & -1.018 & 0.115 & 11/11/2010 5:34:22 PM \\
\hline 20000098174 & 11.61 & 0.379 & -0.335 & 11/11/2010 5:34:22 PM \\
\hline 20000098078 & 6.326 & -1.508 & 1.625 & 11/11/2010 5:34:22 PM \\
\hline 20000100089 & 0.059 & -0.042 & 0.175 & $11 / 11 / 2010$ 5:34:22 PM \\
\hline 20000098170 & -4.595 & -1.018 & 0.108 & 11/11/2010 5:34:23 PM \\
\hline 20000098078 & 6.324 & -1.514 & 1.611 & 11/11/2010 5:34:23 PM \\
\hline
\end{tabular}




\begin{tabular}{|c|c|c|c|c|}
\hline 20000100089 & 0.061 & -0.042 & 0.174 & 11/11/2010 5:34:23 PM \\
\hline 20000098169 & -6.731 & -0.764 & 1.212 & 11/11/2010 5:34:23 PM \\
\hline 20000098170 & -4.6 & -1.023 & 0.112 & 11/11/2010 5:34:23 PM \\
\hline 20000098174 & 11.566 & 0.379 & -0.149 & 11/11/2010 5:34:23 PM \\
\hline 20000098078 & 6.323 & -1.511 & 1.592 & 11/11/2010 5:34:23 PM \\
\hline 20000100089 & 0.051 & -0.046 & 0.188 & 11/11/2010 5:34:23 PM \\
\hline 20000098170 & -4.597 & -1.029 & 0.109 & 11/11/2010 5:34:23 PM \\
\hline 20000098078 & 6.317 & -1.487 & 1.584 & 11/11/2010 5:34:24 PM \\
\hline 20000100089 & 0.05 & -0.048 & 0.193 & 11/11/2010 5:34:24 PM \\
\hline 20000098169 & -12.463 & -0.058 & 2.576 & 11/11/2010 5:34:24 PM \\
\hline 20000098170 & -4.602 & -1.033 & 0.118 & 11/11/2010 5:34:24 PM \\
\hline 20000098174 & 11.672 & 0.432 & -0.393 & 11/11/2010 5:34:24 PM \\
\hline 20000098078 & 6.315 & -1.459 & 1.547 & 11/11/2010 5:34:24 PM \\
\hline 20000100089 & 0.052 & -0.048 & 0.189 & 11/11/2010 5:34:24 PM \\
\hline 20000098170 & -4.603 & -1.035 & 0.121 & 11/11/2010 5:34:24 PM \\
\hline 20000098078 & 6.3 & -1.432 & 1.514 & 11/11/2010 5:34:24 PM \\
\hline 20000100089 & 0.054 & -0.045 & 0.186 & 11/11/2010 5:34:24 PM \\
\hline 20000098169 & -12.461 & 0.039 & 2.528 & 11/11/2010 5:34:25 PM \\
\hline 20000098170 & -4.607 & -1.033 & 0.129 & 11/11/2010 5:34:25 PM \\
\hline 20000098174 & 11.617 & 0.386 & -0.28 & 11/11/2010 5:34:25 PM \\
\hline 20000098078 & 6.301 & -1.427 & 1.484 & 11/11/2010 5:34:25 PM \\
\hline 20000100089 & 0.052 & -0.043 & 0.184 & 11/11/2010 5:34:25 PM \\
\hline 20000098170 & -4.608 & -1.027 & 0.142 & 11/11/2010 5:34:25 PM \\
\hline 20000098078 & 6.309 & -1.435 & 1.469 & 11/11/2010 5:34:25 PM \\
\hline 20000100089 & 0.05 & -0.042 & 0.177 & $11 / 11 / 2010$ 5:34:25 PM \\
\hline 20000098169 & -5.239 & -0.928 & 1.314 & 11/11/2010 5:34:25 PM \\
\hline 20000098170 & -4.604 & -1.025 & 0.147 & 11/11/2010 5:34:26 PM \\
\hline 20000098174 & 11.633 & 0.127 & -0.456 & 11/11/2010 5:34:26 PM \\
\hline 20000098078 & 6.199 & -1.142 & 1.633 & 11/11/2010 5:34:26 PM \\
\hline 20000100089 & 0.049 & -0.041 & 0.164 & $11 / 11 / 2010$ 5:34:26 PM \\
\hline 20000098170 & -4.597 & -1.023 & 0.147 & 11/11/2010 5:34:26 PM \\
\hline 20000098078 & 6.207 & -1.145 & 1.633 & 11/11/2010 5:34:26 PM \\
\hline 20000100089 & 0.056 & -0.04 & 0.161 & 11/11/2010 5:34:26 PM \\
\hline 20000098169 & -5.834 & -0.883 & 1.405 & 11/11/2010 5:34:26 PM \\
\hline 20000098170 & -4.602 & -1.019 & 0.152 & 11/11/2010 5:34:26 PM \\
\hline 20000098174 & 11.595 & 0.337 & -0.301 & 11/11/2010 5:34:26 PM \\
\hline 20000098078 & 6.218 & -1.161 & 1.607 & 11/11/2010 5:34:27 PM \\
\hline 20000100089 & 0.054 & -0.039 & 0.15 & 11/11/2010 5:34:27 PM \\
\hline 20000098170 & -4.61 & -1.013 & 0.154 & 11/11/2010 5:34:27 PM \\
\hline 20000098078 & 6.359 & -1.376 & 1.171 & 11/11/2010 5:34:27 PM \\
\hline 20000100089 & 0.055 & -0.041 & 0.144 & $11 / 11 / 2010$ 5:34:27 PM \\
\hline 20000098169 & -7.011 & -0.692 & 1.125 & $11 / 11 / 2010$ 5:34:27 PM \\
\hline 20000098170 & -4.615 & -1.013 & 0.159 & 11/11/2010 5:34:27 PM \\
\hline 20000098174 & 11.55 & 0.287 & -0.152 & $11 / 11 / 2010$ 5:34:27 PM \\
\hline 20000098078 & 6.355 & -1.364 & 1.176 & $11 / 11 / 2010$ 5:34:27 PM \\
\hline 20000100089 & 0.057 & -0.042 & 0.132 & 11/11/2010 5:34:27 PM \\
\hline 20000098170 & -4.615 & -1.014 & 0.154 & 11/11/2010 5:34:28 PM \\
\hline
\end{tabular}




\begin{tabular}{|c|c|c|c|c|}
\hline 20000098078 & 6.354 & -1.383 & 1.176 & 11/11/2010 5:34:28 PM \\
\hline 20000100089 & 0.058 & -0.04 & 0.123 & 11/11/2010 5:34:28 PM \\
\hline 20000098169 & -5.303 & -0.938 & 1.348 & 11/11/2010 5:34:28 PM \\
\hline 20000098170 & -4.61 & -1.012 & 0.146 & 11/11/2010 5:34:28 PM \\
\hline 20000098174 & 11.564 & 0.379 & -0.146 & 11/11/2010 5:34:28 PM \\
\hline 20000098078 & 6.349 & -1.391 & 1.157 & 11/11/2010 5:34:28 PM \\
\hline 20000100089 & 0.059 & -0.04 & 0.113 & 11/11/2010 5:34:28 PM \\
\hline 20000098170 & -4.605 & -1.007 & 0.133 & 11/11/2010 5:34:29 PM \\
\hline 20000098078 & 6.341 & -1.388 & 1.151 & $11 / 11 / 2010$ 5:34:29 PM \\
\hline 20000100089 & 0.055 & -0.041 & 0.109 & 11/11/2010 5:34:29 PM \\
\hline 20000098169 & -12.474 & 0.029 & 2.552 & 11/11/2010 5:34:29 PM \\
\hline 20000098170 & -4.604 & -1.008 & 0.135 & 11/11/2010 5:34:29 PM \\
\hline 20000098174 & 11.57 & 0.244 & -0.247 & $11 / 11 / 2010$ 5:34:29 PM \\
\hline 20000098078 & 6.341 & -1.391 & 1.139 & 11/11/2010 5:34:29 PM \\
\hline 20000100089 & 0.055 & -0.041 & 0.109 & $11 / 11 / 2010$ 5:34:29 PM \\
\hline 20000098170 & -4.602 & -1.009 & 0.132 & 11/11/2010 5:34:29 PM \\
\hline 20000098078 & 6.337 & -1.392 & 1.116 & 11/11/2010 5:34:30 PM \\
\hline 20000100089 & 0.048 & -0.037 & 0.104 & 11/11/2010 5:34:30 PM \\
\hline 20000098169 & -5.934 & -0.818 & 1.636 & 11/11/2010 5:34:30 PM \\
\hline 20000098170 & -4.598 & -1.009 & 0.122 & 11/11/2010 5:34:30 PM \\
\hline 20000098174 & 11.588 & 0.229 & -0.321 & 11/11/2010 5:34:30 PM \\
\hline 20000098078 & 6.341 & -1.4 & 1.083 & 11/11/2010 5:34:30 PM \\
\hline 20000100089 & 0.046 & -0.035 & 0.097 & $11 / 11 / 20105: 34: 30 \mathrm{PM}$ \\
\hline 20000098170 & -4.595 & -1.01 & 0.112 & 11/11/2010 5:34:30 PM \\
\hline 20000098078 & 6.336 & -1.405 & 1.043 & 11/11/2010 5:34:30 PM \\
\hline 20000100089 & 0.044 & -0.032 & 0.09 & 11/11/2010 5:34:30 PM \\
\hline 20000098169 & -5.264 & -0.901 & 1.247 & 11/11/2010 5:34:31 PM \\
\hline 20000098170 & -4.596 & -1.009 & 0.103 & 11/11/2010 5:34:31 PM \\
\hline 20000098174 & 11.572 & 0.317 & -0.238 & 11/11/2010 5:34:31 PM \\
\hline 20000098078 & 6.337 & -1.413 & 1.023 & 11/11/2010 5:34:31 PM \\
\hline 20000100089 & 0.049 & -0.031 & 0.096 & 11/11/2010 5:34:31 PM \\
\hline 20000098078 & 6.34 & -1.422 & 1.001 & 11/11/2010 5:34:31 PM \\
\hline 20000100089 & 0.051 & -0.029 & 0.088 & 11/11/2010 5:34:31 PM \\
\hline 20000098169 & -6.028 & -0.556 & 1.598 & 11/11/2010 5:34:31 PM \\
\hline 20000098170 & -4.601 & -1.016 & 0.113 & 11/11/2010 5:34:32 PM \\
\hline 20000098174 & 11.611 & 0.343 & -0.228 & 11/11/2010 5:34:32 PM \\
\hline 20000098078 & 6.342 & -1.423 & 0.97 & 11/11/2010 5:34:32 PM \\
\hline 20000100089 & 0.053 & -0.027 & 0.085 & $11 / 11 / 2010$ 5:34:32 PM \\
\hline 20000098170 & -4.601 & -1.019 & 0.113 & 11/11/2010 5:34:32 PM \\
\hline 20000098078 & 6.298 & -1.226 & 1.22 & 11/11/2010 5:34:32 PM \\
\hline 20000100089 & 0.052 & -0.026 & 0.085 & 11/11/2010 5:34:32 PM \\
\hline 20000098169 & -7.194 & -0.828 & 1.298 & $11 / 11 / 2010$ 5:34:32 PM \\
\hline 20000098170 & -4.607 & -1.019 & 0.106 & 11/11/2010 5:34:32 PM \\
\hline 20000098174 & 11.578 & 0.253 & -0.223 & 11/11/2010 5:34:33 PM \\
\hline 20000098078 & 6.308 & -1.236 & 1.203 & $11 / 11 / 2010$ 5:34:33 PM \\
\hline 20000100089 & 0.041 & -0.03 & 0.1 & 11/11/2010 5:34:33 PM \\
\hline 20000098170 & -4.604 & -1.022 & 0.126 & 11/11/2010 5:34:33 PM \\
\hline
\end{tabular}




\begin{tabular}{|c|c|c|c|c|}
\hline 20000098078 & 6.315 & -1.251 & 1.184 & 11/11/2010 5:34:33 PM \\
\hline 20000100089 & 0.044 & -0.032 & 0.101 & 11/11/2010 5:34:33 PM \\
\hline 20000098169 & -3.607 & -0.858 & 2.032 & 11/11/2010 5:34:33 PM \\
\hline 20000098170 & -4.61 & -1.028 & 0.136 & 11/11/2010 5:34:33 PM \\
\hline 20000098174 & 11.589 & 0.277 & -0.178 & 11/11/2010 5:34:33 PM \\
\hline 20000098078 & 6.315 & -1.263 & 1.172 & 11/11/2010 5:34:33 PM \\
\hline 20000100089 & 0.05 & -0.037 & 0.096 & 11/11/2010 5:34:34 PM \\
\hline 20000098170 & -4.607 & -1.033 & 0.134 & 11/11/2010 5:34:34 PM \\
\hline 20000098078 & 6.318 & -1.28 & 1.149 & 11/11/2010 5:34:34 PM \\
\hline 20000100089 & 0.049 & -0.037 & 0.091 & 11/11/2010 5:34:34 PM \\
\hline 20000098169 & -6.872 & -0.797 & 1.393 & 11/11/2010 5:34:34 PM \\
\hline 20000098170 & -4.599 & -1.03 & 0.128 & 11/11/2010 5:34:34 PM \\
\hline 20000098174 & 11.586 & 0.263 & -0.255 & 11/11/2010 5:34:34 PM \\
\hline 20000098078 & 6.313 & -1.283 & 1.142 & 11/11/2010 5:34:34 PM \\
\hline 20000100089 & 0.051 & -0.037 & 0.089 & 11/11/2010 5:34:34 PM \\
\hline 20000098170 & -4.606 & -1.022 & 0.12 & 11/11/2010 5:34:35 PM \\
\hline 20000098078 & 6.32 & -1.292 & 1.125 & 11/11/2010 5:34:35 PM \\
\hline 20000100089 & 0.048 & -0.035 & 0.083 & 11/11/2010 5:34:35 PM \\
\hline 20000098169 & -5.488 & -0.85 & 1.527 & 11/11/2010 5:34:35 PM \\
\hline 20000098174 & 11.59 & 0.385 & -0.161 & 11/11/2010 5:34:35 PM \\
\hline 20000098078 & 6.321 & -1.293 & 1.132 & 11/11/2010 5:34:35 PM \\
\hline 20000098078 & 6.32 & -1.292 & 1.165 & 11/11/2010 5:34:36 PM \\
\hline 20000100089 & 0.041 & -0.037 & 0.079 & $11 / 11 / 2010$ 5:34:36 PM \\
\hline 20000098169 & -12.475 & 0.029 & 2.649 & 11/11/2010 5:34:36 PM \\
\hline 20000098170 & -4.609 & -1.019 & 0.109 & 11/11/2010 5:34:36 PM \\
\hline 20000098174 & 11.548 & 0.342 & -0.141 & 11/11/2010 5:34:36 PM \\
\hline 20000098078 & 6.307 & -1.287 & 1.169 & 11/11/2010 5:34:36 PM \\
\hline 20000100089 & 0.037 & -0.034 & 0.071 & 11/11/2010 5:34:36 PM \\
\hline 20000098078 & 6.314 & -1.294 & 1.145 & 11/11/2010 5:34:36 PM \\
\hline 20000098169 & -6.724 & -0.851 & 1.285 & 11/11/2010 5:34:37 PM \\
\hline 20000098174 & 11.596 & 0.377 & -0.217 & 11/11/2010 5:34:37 PM \\
\hline 20000098078 & 6.307 & -1.295 & 1.126 & 11/11/2010 5:34:37 PM \\
\hline 20000098170 & -3.615 & -0.794 & 1.008 & 11/11/2010 5:34:37 PM \\
\hline 20000098078 & 6.3 & -1.308 & 1.101 & 11/11/2010 5:34:37 PM \\
\hline 20000098169 & -7.58 & -0.71 & 1.133 & 11/11/2010 5:34:38 PM \\
\hline 20000098170 & -4.456 & -1.082 & 0.628 & 11/11/2010 5:34:38 PM \\
\hline 20000098174 & 11.648 & 0.388 & -0.312 & 11/11/2010 5:34:38 PM \\
\hline 20000098078 & 6.299 & -1.318 & 1.066 & $11 / 11 / 2010$ 5:34:38 PM \\
\hline 20000098170 & -4.442 & -1.078 & 0.61 & 11/11/2010 5:34:38 PM \\
\hline 20000098078 & 6.3 & -1.328 & 1.042 & 11/11/2010 5:34:38 PM \\
\hline 20000098170 & -4.463 & -1.082 & 0.577 & $11 / 11 / 2010$ 5:34:38 PM \\
\hline 20000098174 & 11.59 & 0.315 & -0.263 & 11/11/2010 5:34:39 PM \\
\hline 20000098078 & 6.3 & -1.336 & 1.029 & 11/11/2010 5:34:39 PM \\
\hline 20000100089 & 0.032 & -0.035 & 0.067 & $11 / 11 / 2010$ 5:34:39 PM \\
\hline 20000098170 & -4.48 & -1.078 & 0.545 & $11 / 11 / 2010$ 5:34:39 PM \\
\hline 20000098078 & 6.304 & -1.36 & 1.029 & 11/11/2010 5:34:39 PM \\
\hline 20000098169 & -5.58 & -0.891 & 1.601 & 11/11/2010 5:34:39 PM \\
\hline
\end{tabular}




\begin{tabular}{|c|c|c|c|c|}
\hline 20000098170 & -4.495 & -1.075 & 0.516 & 11/11/2010 5:34:39 PM \\
\hline 20000098174 & 11.669 & 0.505 & -0.447 & 11/11/2010 5:34:39 PM \\
\hline 20000098078 & 6.3 & -1.363 & 1.033 & 11/11/2010 5:34:40 PM \\
\hline 20000098170 & -4.501 & -1.077 & 0.482 & 11/11/2010 5:34:40 PM \\
\hline 20000098078 & 6.311 & -1.383 & 1.051 & 11/11/2010 5:34:40 PM \\
\hline 20000098169 & -12.485 & 0.051 & 2.386 & 11/11/2010 5:34:40 PM \\
\hline 20000098170 & -4.509 & -1.067 & 0.455 & 11/11/2010 5:34:40 PM \\
\hline 20000098174 & 11.561 & 0.284 & -0.148 & 11/11/2010 5:34:40 PM \\
\hline 20000098078 & 6.314 & -1.396 & 1.055 & 11/11/2010 5:34:40 PM \\
\hline 20000098170 & -4.519 & -1.051 & 0.426 & 11/11/2010 5:34:41 PM \\
\hline 20000098078 & 6.322 & -1.415 & 1.066 & 11/11/2010 5:34:41 PM \\
\hline 20000098169 & -12.485 & 0.08 & 2.384 & 11/11/2010 5:34:41 PM \\
\hline 20000098170 & -4.527 & -1.041 & 0.394 & 11/11/2010 5:34:41 PM \\
\hline 20000098174 & 11.548 & 0.365 & -0.204 & 11/11/2010 5:34:41 PM \\
\hline 20000098078 & 6.321 & -1.434 & 1.107 & 11/11/2010 5:34:41 PM \\
\hline 20000098170 & -4.528 & -1.033 & 0.361 & $11 / 11 / 2010$ 5:34:42 PM \\
\hline 20000098078 & 6.317 & -1.626 & 1.599 & 11/11/2010 5:34:42 PM \\
\hline 20000098169 & -6.56 & -0.902 & 1.241 & 11/11/2010 5:34:42 PM \\
\hline 20000098170 & -4.522 & -1.029 & 0.337 & 11/11/2010 5:34:42 PM \\
\hline 20000098174 & 11.604 & 0.239 & -0.291 & 11/11/2010 5:34:42 PM \\
\hline 20000098078 & 6.317 & -1.621 & 1.568 & 11/11/2010 5:34:42 PM \\
\hline 20000098170 & -4.534 & -1.025 & 0.321 & 11/11/2010 5:34:42 PM \\
\hline 20000098078 & 6.318 & -1.613 & 1.557 & 11/11/2010 5:34:43 PM \\
\hline 20000098169 & -6.388 & -0.922 & 1.202 & 11/11/2010 5:34:43 PM \\
\hline 20000098170 & -4.538 & -1.023 & 0.295 & 11/11/2010 5:34:43 PM \\
\hline 20000098174 & 11.581 & 0.309 & -0.19 & 11/11/2010 5:34:43 PM \\
\hline 20000098078 & 6.321 & -1.602 & 1.526 & 11/11/2010 5:34:43 PM \\
\hline 20000098170 & -4.554 & -1.016 & 0.28 & $11 / 11 / 2010$ 5:34:43 PM \\
\hline 20000098078 & 6.327 & -1.422 & 0.964 & 11/11/2010 5:34:43 PM \\
\hline 20000098169 & -12.456 & 0.009 & 2.615 & $11 / 11 / 2010$ 5:34:44 PM \\
\hline 20000098170 & -4.563 & -1.015 & 0.258 & $11 / 11 / 20105: 34: 44$ PM \\
\hline 20000098174 & 11.616 & 0.378 & -0.195 & 11/11/2010 5:34:44 PM \\
\hline 20000098078 & 6.334 & -1.422 & 0.952 & 11/11/2010 5:34:44 PM \\
\hline 20000098170 & -4.577 & -1.023 & 0.241 & 11/11/2010 5:34:44 PM \\
\hline 20000098078 & 6.335 & -1.428 & 0.941 & $11 / 11 / 2010$ 5:34:44 PM \\
\hline 20000098169 & -6.335 & -0.942 & 1.237 & 11/11/2010 5:34:44 PM \\
\hline 20000098170 & -4.584 & -1.029 & 0.228 & 11/11/2010 5:34:45 PM \\
\hline 20000098174 & 11.629 & 0.183 & -0.429 & $11 / 11 / 2010$ 5:34:45 PM \\
\hline 20000098078 & 6.338 & -1.447 & 0.962 & 11/11/2010 5:34:45 PM \\
\hline 20000100089 & 0.038 & -0.035 & 0.067 & 11/11/2010 5:34:45 PM \\
\hline 20000098170 & -4.592 & -1.032 & 0.218 & 11/11/2010 5:34:45 PM \\
\hline 20000098078 & 6.335 & -1.464 & 0.986 & $11 / 11 / 2010$ 5:34:45 PM \\
\hline 20000100089 & 0.043 & -0.036 & 0.066 & 11/11/2010 5:34:45 PM \\
\hline 20000098169 & -6.807 & -0.815 & 1.084 & 11/11/2010 5:34:45 PM \\
\hline 20000098170 & -4.594 & -1.022 & 0.214 & $11 / 11 / 2010$ 5:34:45 PM \\
\hline 20000098174 & 11.542 & 0.187 & -0.166 & 11/11/2010 5:34:46 PM \\
\hline 20000098078 & 6.335 & -1.467 & 0.992 & 11/11/2010 5:34:46 PM \\
\hline
\end{tabular}




\begin{tabular}{|c|c|c|c|c|}
\hline 20000100089 & 0.046 & -0.033 & 0.062 & 11/11/2010 5:34:46 PM \\
\hline 20000098170 & -4.593 & -1.017 & 0.203 & 11/11/2010 5:34:46 PM \\
\hline 20000098078 & 6.329 & -1.459 & 1.002 & 11/11/2010 5:34:46 PM \\
\hline 20000100089 & 0.049 & -0.034 & 0.06 & 11/11/2010 5:34:46 PM \\
\hline 20000098169 & -6.306 & -0.899 & 1.233 & 11/11/2010 5:34:46 PM \\
\hline 20000098170 & -4.589 & -1.01 & 0.191 & 11/11/2010 5:34:46 PM \\
\hline 20000098174 & 11.594 & 0.357 & -0.236 & 11/11/2010 5:34:46 PM \\
\hline 20000098078 & 6.326 & -1.453 & 0.99 & 11/11/2010 5:34:46 PM \\
\hline 20000100089 & 0.05 & -0.033 & 0.055 & 11/11/2010 5:34:46 PM \\
\hline 20000098170 & -4.591 & -1.003 & 0.19 & 11/11/2010 5:34:47 PM \\
\hline 20000098078 & 6.328 & -1.452 & 1.019 & 11/11/2010 5:34:47 PM \\
\hline 20000100089 & 0.051 & -0.033 & 0.056 & 11/11/2010 5:34:47 PM \\
\hline 20000098169 & -12.468 & -0.046 & 2.509 & 11/11/2010 5:34:47 PM \\
\hline 20000098170 & -4.59 & -1.002 & 0.197 & 11/11/2010 5:34:47 PM \\
\hline 20000098174 & 11.541 & 0.296 & -0.087 & 11/11/2010 5:34:47 PM \\
\hline 20000098078 & 6.333 & -1.453 & 1.029 & 11/11/2010 5:34:47 PM \\
\hline 20000098170 & -4.583 & -1.004 & 0.195 & 11/11/2010 5:34:48 PM \\
\hline 20000098078 & 6.33 & -1.45 & 1.039 & 11/11/2010 5:34:48 PM \\
\hline 20000100089 & 0.049 & -0.033 & 0.056 & 11/11/2010 5:34:48 PM \\
\hline 20000098169 & -4.333 & -0.859 & 1.662 & 11/11/2010 5:34:48 PM \\
\hline 20000098170 & -4.575 & -1.009 & 0.194 & 11/11/2010 5:34:48 PM \\
\hline 20000098078 & 6.333 & -1.441 & 1.038 & 11/11/2010 5:34:48 PM \\
\hline 20000100089 & 0.052 & -0.034 & 0.059 & 11/11/2010 5:34:48 PM \\
\hline 20000098170 & -4.571 & -1.015 & 0.189 & 11/11/2010 5:34:48 PM \\
\hline 20000098078 & 6.327 & -1.455 & 1.071 & 11/11/2010 5:34:49 PM \\
\hline 20000100089 & 0.051 & -0.034 & 0.064 & 11/11/2010 5:34:49 PM \\
\hline 20000098169 & -6.396 & -0.889 & 1.116 & 11/11/2010 5:34:49 PM \\
\hline 20000098170 & -4.568 & -1.018 & 0.18 & 11/11/2010 5:34:49 PM \\
\hline 20000098174 & 11.551 & 0.289 & -0.106 & 11/11/2010 5:34:49 PM \\
\hline 20000098078 & 6.325 & -1.462 & 1.074 & 11/11/2010 5:34:49 PM \\
\hline 20000100089 & 0.055 & -0.033 & 0.061 & 11/11/2010 5:34:49 PM \\
\hline 20000098170 & -4.564 & -1.018 & 0.172 & 11/11/2010 5:34:49 PM \\
\hline 20000098078 & 6.315 & -1.455 & 1.082 & 11/11/2010 5:34:49 PM \\
\hline 20000100089 & 0.057 & -0.03 & 0.06 & $11 / 11 / 2010$ 5:34:50 PM \\
\hline 20000098169 & -5.205 & -0.664 & 1.513 & 11/11/2010 5:34:50 PM \\
\hline 20000098170 & -4.569 & -1.014 & 0.171 & 11/11/2010 5:34:50 PM \\
\hline 20000098174 & 11.621 & 0.397 & -0.197 & 11/11/2010 5:34:50 PM \\
\hline 20000098078 & 6.306 & -1.449 & 1.082 & $11 / 11 / 20105: 34: 50 \mathrm{PM}$ \\
\hline 20000100089 & 0.055 & -0.031 & 0.056 & 11/11/2010 5:34:50 PM \\
\hline 20000098170 & -4.577 & -1.007 & 0.169 & 11/11/2010 5:34:50 PM \\
\hline 20000098078 & 6.274 & -1.536 & 1.519 & $11 / 11 / 2010$ 5:34:50 PM \\
\hline 20000100089 & 0.055 & -0.03 & 0.061 & $11 / 11 / 2010$ 5:34:50 PM \\
\hline 20000098169 & -12.462 & 0.03 & 2.676 & 11/11/2010 5:34:50 PM \\
\hline 20000098170 & -4.579 & -1.005 & 0.161 & 11/11/2010 5:34:51 PM \\
\hline 20000098174 & 11.529 & 0.372 & -0.092 & $11 / 11 / 2010$ 5:34:51 PM \\
\hline 20000098078 & 6.275 & -1.525 & 1.511 & 11/11/2010 5:34:51 PM \\
\hline 20000100089 & 0.06 & -0.029 & 0.08 & 11/11/2010 5:34:51 PM \\
\hline
\end{tabular}




\begin{tabular}{|c|c|c|c|c|}
\hline 20000098170 & -4.583 & -1.006 & 0.149 & 11/11/2010 5:34:51 PM \\
\hline 20000098078 & 6.277 & -1.524 & 1.503 & 11/11/2010 5:34:51 PM \\
\hline 20000100089 & 0.061 & -0.028 & 0.087 & 11/11/2010 5:34:51 PM \\
\hline 20000098169 & -6.851 & -0.695 & 1.266 & 11/11/2010 5:34:51 PM \\
\hline 20000098170 & -4.584 & -1.006 & 0.139 & 11/11/2010 5:34:51 PM \\
\hline 20000098174 & 11.568 & 0.326 & -0.205 & 11/11/2010 5:34:52 PM \\
\hline 20000098078 & 6.282 & -1.517 & 1.458 & 11/11/2010 5:34:52 PM \\
\hline 20000100089 & 0.06 & -0.029 & 0.089 & 11/11/2010 5:34:52 PM \\
\hline 20000098170 & -4.587 & -1.005 & 0.142 & $11 / 11 / 2010$ 5:34:52 PM \\
\hline 20000098078 & 6.293 & -1.506 & 1.42 & 11/11/2010 5:34:52 PM \\
\hline 20000098169 & -12.487 & -0.036 & 2.528 & 11/11/2010 5:34:52 PM \\
\hline 20000098170 & -4.586 & -1.009 & 0.139 & 11/11/2010 5:34:52 PM \\
\hline 20000098174 & 11.59 & 0.314 & -0.252 & $11 / 11 / 2010$ 5:34:52 PM \\
\hline 20000098078 & 6.293 & -1.497 & 1.392 & 11/11/2010 5:34:52 PM \\
\hline 20000100089 & 0.056 & -0.031 & 0.08 & 11/11/2010 5:34:53 PM \\
\hline 20000098170 & -4.578 & -1.007 & 0.135 & $11 / 11 / 2010$ 5:34:53 PM \\
\hline 20000098078 & 6.288 & -1.474 & 1.356 & 11/11/2010 5:34:53 PM \\
\hline 20000100089 & 0.056 & -0.031 & 0.083 & 11/11/2010 5:34:53 PM \\
\hline 20000098170 & -4.565 & -1.003 & 0.131 & 11/11/2010 5:34:53 PM \\
\hline 20000098174 & 11.578 & 0.285 & -0.248 & 11/11/2010 5:34:53 PM \\
\hline 20000098078 & 6.298 & -1.472 & 1.333 & 11/11/2010 5:34:53 PM \\
\hline 20000100089 & 0.051 & -0.032 & 0.089 & 11/11/2010 5:34:53 PM \\
\hline 20000098170 & -4.561 & -1.001 & 0.125 & $11 / 11 / 2010$ 5:34:54 PM \\
\hline 20000098078 & 6.284 & -1.455 & 1.307 & 11/11/2010 5:34:54 PM \\
\hline 20000100089 & 0.05 & -0.035 & 0.096 & 11/11/2010 5:34:54 PM \\
\hline 20000098169 & -6.964 & -0.748 & 1.285 & 11/11/2010 5:34:54 PM \\
\hline 20000098170 & -4.561 & -0.999 & 0.115 & 11/11/2010 5:34:54 PM \\
\hline 20000098078 & 6.28 & -1.441 & 1.289 & $11 / 11 / 2010$ 5:34:54 PM \\
\hline 20000100089 & 0.051 & -0.037 & 0.101 & 11/11/2010 5:34:54 PM \\
\hline 20000098170 & -4.56 & -0.991 & 0.122 & 11/11/2010 5:34:54 PM \\
\hline 20000098078 & 6.278 & -1.431 & 1.269 & 11/11/2010 5:34:55 PM \\
\hline 20000100089 & 0.047 & -0.041 & 0.107 & 11/11/2010 5:34:55 PM \\
\hline 20000098169 & -6.336 & -1.013 & 1.212 & 11/11/2010 5:34:55 PM \\
\hline 20000098170 & -4.557 & -0.99 & 0.127 & 11/11/2010 5:34:55 PM \\
\hline 20000098174 & 11.622 & 0.267 & -0.422 & 11/11/2010 5:34:55 PM \\
\hline 20000098078 & 6.288 & -1.432 & 1.235 & 11/11/2010 5:34:55 PM \\
\hline 20000100089 & 0.051 & -0.043 & 0.118 & 11/11/2010 5:34:55 PM \\
\hline 20000098170 & -4.554 & -0.991 & 0.126 & $11 / 11 / 2010$ 5:34:55 PM \\
\hline 20000098078 & 6.296 & -1.438 & 1.195 & 11/11/2010 5:34:56 PM \\
\hline 20000100089 & 0.062 & -0.044 & 0.128 & 11/11/2010 5:34:56 PM \\
\hline 20000098169 & -6.136 & -0.969 & 1.223 & 11/11/2010 5:34:56 PM \\
\hline 20000098170 & -4.554 & -0.989 & 0.122 & $11 / 11 / 2010$ 5:34:56 PM \\
\hline 20000098174 & 11.575 & 0.279 & -0.216 & 11/11/2010 5:34:56 PM \\
\hline 20000098078 & 6.303 & -1.44 & 1.177 & 11/11/2010 5:34:56 PM \\
\hline 20000100089 & 0.064 & -0.044 & 0.151 & $11 / 11 / 2010$ 5:34:56 PM \\
\hline 20000098170 & -4.554 & -0.983 & 0.131 & 11/11/2010 5:34:56 PM \\
\hline 20000098078 & 6.309 & -1.454 & 1.171 & 11/11/2010 5:34:56 PM \\
\hline
\end{tabular}




\begin{tabular}{|c|c|c|c|c|}
\hline 20000100089 & 0.073 & -0.047 & 0.178 & 11/11/2010 5:34:56 PM \\
\hline 20000098169 & -6.68 & -0.823 & 1.478 & 11/11/2010 5:34:57 PM \\
\hline 20000098170 & -4.564 & -0.99 & 0.14 & 11/11/2010 5:34:57 PM \\
\hline 20000098174 & 11.557 & 0.312 & -0.142 & 11/11/2010 5:34:57 PM \\
\hline 20000098078 & 6.305 & -1.454 & 1.169 & 11/11/2010 5:34:57 PM \\
\hline 20000100089 & 0.079 & -0.053 & 0.176 & 11/11/2010 5:34:57 PM \\
\hline 20000098170 & -4.567 & -0.993 & 0.14 & 11/11/2010 5:34:57 PM \\
\hline 20000098078 & 6.302 & -1.448 & 1.141 & 11/11/2010 5:34:57 PM \\
\hline 20000100089 & 0.078 & -0.047 & 0.162 & 11/11/2010 5:34:57 PM \\
\hline 20000098169 & -5.725 & -0.846 & 1.549 & 11/11/2010 5:34:57 PM \\
\hline 20000098170 & -4.57 & -0.997 & 0.135 & 11/11/2010 5:34:58 PM \\
\hline 20000098174 & 11.63 & 0.371 & -0.298 & 11/11/2010 5:34:58 PM \\
\hline 20000098078 & 6.305 & -1.443 & 1.109 & 11/11/2010 5:34:58 PM \\
\hline 20000098170 & -4.588 & -1.005 & 0.138 & 11/11/2010 5:34:58 PM \\
\hline 20000098078 & 6.312 & -1.444 & 1.07 & $11 / 11 / 2010$ 5:34:58 PM \\
\hline 20000098169 & -5.639 & -0.817 & 1.521 & 11/11/2010 5:34:58 PM \\
\hline 20000098170 & -4.598 & -1.015 & 0.145 & 11/11/2010 5:34:58 PM \\
\hline 20000098174 & 11.579 & 0.37 & -0.215 & 11/11/2010 5:34:58 PM \\
\hline 20000098078 & 6.31 & -1.433 & 1.049 & 11/11/2010 5:34:59 PM \\
\hline 20000100089 & 0.078 & -0.044 & 0.149 & 11/11/2010 5:34:59 PM \\
\hline 20000098170 & -4.603 & -1.02 & 0.157 & 11/11/2010 5:34:59 PM \\
\hline 20000098078 & 6.307 & -1.42 & 1.042 & 11/11/2010 5:34:59 PM \\
\hline 20000100089 & 0.075 & -0.044 & 0.136 & $11 / 11 / 2010$ 5:34:59 PM \\
\hline 20000098169 & -5.653 & -0.846 & 1.539 & 11/11/2010 5:34:59 PM \\
\hline 20000098170 & -4.599 & -1.023 & 0.157 & 11/11/2010 5:34:59 PM \\
\hline 20000098174 & 11.601 & 0.37 & -0.178 & 11/11/2010 5:34:59 PM \\
\hline 20000098078 & 6.305 & -1.41 & 1.039 & 11/11/2010 5:34:59 PM \\
\hline 20000098170 & -4.594 & -1.025 & 0.153 & 11/11/2010 5:35:00 PM \\
\hline 20000098078 & 6.311 & -1.406 & 1.025 & 11/11/2010 5:35:00 PM \\
\hline 20000100089 & 0.069 & -0.043 & 0.125 & $11 / 11 / 2010$ 5:35:00 PM \\
\hline 20000098169 & -5.396 & -0.948 & 1.384 & 11/11/2010 5:35:00 PM \\
\hline 20000098170 & -4.591 & -1.027 & 0.145 & 11/11/2010 5:35:00 PM \\
\hline 20000098174 & 11.576 & 0.347 & -0.178 & 11/11/2010 5:35:00 PM \\
\hline 20000098078 & 6.306 & -1.396 & 1.035 & 11/11/2010 5:35:00 PM \\
\hline 20000098170 & -4.583 & -1.022 & 0.139 & 11/11/2010 5:35:01 PM \\
\hline 20000098078 & 6.303 & -1.389 & 1.015 & 11/11/2010 5:35:01 PM \\
\hline 20000098169 & -7.882 & -0.599 & 1.194 & 11/11/2010 5:35:01 PM \\
\hline 20000098170 & -4.59 & -1.015 & 0.131 & $11 / 11 / 2010$ 5:35:01 PM \\
\hline 20000098174 & 11.591 & 0.328 & -0.276 & 11/11/2010 5:35:01 PM \\
\hline 20000098078 & 6.264 & -1.305 & 1.273 & 11/11/2010 5:35:01 PM \\
\hline 20000098170 & -4.595 & -1.009 & 0.121 & 11/11/2010 5:35:01 PM \\
\hline 20000098078 & 6.27 & -1.315 & 1.282 & $11 / 11 / 2010$ 5:35:02 PM \\
\hline 20000100089 & 0.067 & -0.045 & 0.026 & 11/11/2010 5:35:02 PM \\
\hline 20000098169 & -12.481 & 0.109 & 2.602 & 11/11/2010 5:35:02 PM \\
\hline 20000098170 & -4.6 & -1.009 & 0.11 & $11 / 11 / 2010$ 5:35:02 PM \\
\hline 20000098174 & 11.577 & 0.271 & -0.167 & 11/11/2010 5:35:02 PM \\
\hline 20000098078 & 6.26 & -1.312 & 1.279 & 11/11/2010 5:35:02 PM \\
\hline
\end{tabular}




\begin{tabular}{|c|c|c|c|c|}
\hline 20000100089 & 0.062 & -0.046 & 0.033 & 11/11/2010 5:35:02 PM \\
\hline 20000098078 & 6.3 & -1.351 & 0.292 & 11/11/2010 5:35:02 PM \\
\hline 20000100089 & 0.066 & -0.044 & 0.036 & 11/11/2010 5:35:02 PM \\
\hline 20000098169 & -6.303 & -0.925 & 1.187 & 11/11/2010 5:35:03 PM \\
\hline 20000098174 & 11.6 & 0.326 & -0.197 & 11/11/2010 5:35:03 PM \\
\hline 20000098078 & 6.33 & -1.254 & 0.801 & 11/11/2010 5:35:03 PM \\
\hline 20000100089 & 0.065 & -0.039 & 0.033 & 11/11/2010 5:35:03 PM \\
\hline 20000098078 & 6.324 & -1.252 & 0.795 & 11/11/2010 5:35:03 PM \\
\hline 20000098169 & -5.879 & -0.873 & 1.355 & 11/11/2010 5:35:03 PM \\
\hline 20000098174 & 11.58 & 0.355 & -0.181 & 11/11/2010 5:35:04 PM \\
\hline 20000098078 & 6.328 & -1.261 & 0.795 & 11/11/2010 5:35:04 PM \\
\hline 20000098170 & -4.597 & -1.001 & 0.128 & 11/11/2010 5:35:04 PM \\
\hline 20000098078 & 6.332 & -1.275 & 0.804 & 11/11/2010 5:35:04 PM \\
\hline 20000100089 & 0.068 & -0.041 & 0.059 & 11/11/2010 5:35:04 PM \\
\hline 20000098169 & -6.059 & -0.743 & 1.517 & 11/11/2010 5:35:04 PM \\
\hline 20000098170 & -4.6 & -1.001 & 0.143 & 11/11/2010 5:35:04 PM \\
\hline 20000098078 & 6.339 & -1.298 & 0.817 & 11/11/2010 5:35:05 PM \\
\hline 20000100089 & 0.07 & -0.04 & 0.062 & 11/11/2010 5:35:05 PM \\
\hline 20000098170 & -4.596 & -1.003 & 0.146 & 11/11/2010 5:35:05 PM \\
\hline 20000098078 & 6.337 & -1.314 & 0.831 & 11/11/2010 5:35:05 PM \\
\hline 20000100089 & 0.068 & -0.037 & 0.058 & 11/11/2010 5:35:05 PM \\
\hline 20000098169 & -12.443 & 0.026 & 2.609 & 11/11/2010 5:35:05 PM \\
\hline 20000098170 & -4.594 & -1.01 & 0.149 & 11/11/2010 5:35:05 PM \\
\hline 20000098174 & 11.63 & 0.367 & -0.197 & 11/11/2010 5:35:05 PM \\
\hline 20000098078 & 6.334 & -1.33 & 0.829 & 11/11/2010 5:35:05 PM \\
\hline 20000100089 & 0.059 & -0.038 & 0.078 & 11/11/2010 5:35:06 PM \\
\hline 20000098170 & -4.598 & -1.013 & 0.151 & 11/11/2010 5:35:06 PM \\
\hline 20000098078 & 6.332 & -1.357 & 0.856 & 11/11/2010 5:35:06 PM \\
\hline 20000100089 & 0.056 & -0.039 & 0.089 & 11/11/2010 5:35:06 PM \\
\hline 20000098169 & -6.977 & -0.726 & 1.304 & 11/11/2010 5:35:06 PM \\
\hline 20000098170 & -4.602 & -1.015 & 0.158 & 11/11/2010 5:35:06 PM \\
\hline 20000098174 & 11.653 & 0.22 & -0.41 & 11/11/2010 5:35:06 PM \\
\hline 20000098078 & 6.326 & -1.369 & 0.882 & 11/11/2010 5:35:06 PM \\
\hline 20000100089 & 0.052 & -0.038 & 0.107 & 11/11/2010 5:35:06 PM \\
\hline 20000098078 & 6.327 & -1.382 & 0.889 & 11/11/2010 5:35:07 PM \\
\hline 20000100089 & 0.051 & -0.038 & 0.147 & 11/11/2010 5:35:07 PM \\
\hline 20000098169 & -5.586 & -0.771 & 1.532 & 11/11/2010 5:35:07 PM \\
\hline 20000098170 & -4.598 & -1.018 & 0.154 & $11 / 11 / 2010$ 5:35:07 PM \\
\hline 20000098174 & 11.545 & 0.28 & -0.195 & 11/11/2010 5:35:07 PM \\
\hline 20000098078 & 6.321 & -1.374 & 0.896 & 11/11/2010 5:35:07 PM \\
\hline 20000100089 & 0.048 & -0.037 & 0.151 & 11/11/2010 5:35:07 PM \\
\hline 20000098170 & -4.599 & -1.012 & 0.157 & $11 / 11 / 2010$ 5:35:07 PM \\
\hline 20000098078 & 6.314 & -1.37 & 0.904 & 11/11/2010 5:35:08 PM \\
\hline 20000100089 & 0.049 & -0.038 & 0.149 & $11 / 11 / 2010$ 5:35:08 PM \\
\hline 20000098169 & -6.73 & -0.871 & 1.18 & $11 / 11 / 2010$ 5:35:08 PM \\
\hline 20000098170 & -4.604 & -1.004 & 0.16 & 11/11/2010 5:35:08 PM \\
\hline 20000098174 & 11.547 & 0.313 & -0.197 & 11/11/2010 5:35:08 PM \\
\hline
\end{tabular}




\begin{tabular}{|c|c|c|c|c|}
\hline 20000098078 & 6.311 & -1.374 & 0.912 & 11/11/2010 5:35:08 PM \\
\hline 20000100089 & 0.053 & -0.038 & 0.141 & 11/11/2010 5:35:08 PM \\
\hline 20000098170 & -4.606 & -0.995 & 0.169 & 11/11/2010 5:35:08 PM \\
\hline 20000098078 & 6.309 & -1.377 & 0.903 & 11/11/2010 5:35:08 PM \\
\hline 20000100089 & 0.051 & -0.035 & 0.131 & 11/11/2010 5:35:09 PM \\
\hline 20000098169 & -12.48 & -0.003 & 2.543 & 11/11/2010 5:35:09 PM \\
\hline 20000098170 & -4.602 & -0.991 & 0.183 & 11/11/2010 5:35:09 PM \\
\hline 20000098174 & 11.66 & 0.239 & -0.377 & 11/11/2010 5:35:09 PM \\
\hline 20000098078 & 6.313 & -1.37 & 0.907 & 11/11/2010 5:35:09 PM \\
\hline 20000100089 & 0.054 & -0.032 & 0.121 & 11/11/2010 5:35:09 PM \\
\hline 20000098170 & -4.591 & -0.991 & 0.186 & 11/11/2010 5:35:09 PM \\
\hline 20000098078 & 6.315 & -1.371 & 0.911 & 11/11/2010 5:35:09 PM \\
\hline 20000100089 & 0.054 & -0.028 & 0.109 & 11/11/2010 5:35:09 PM \\
\hline 20000098169 & -5.077 & -0.817 & 1.725 & 11/11/2010 5:35:10 PM \\
\hline 20000098170 & -4.58 & -0.994 & 0.184 & 11/11/2010 5:35:10 PM \\
\hline 20000098174 & 11.604 & 0.374 & -0.257 & $11 / 11 / 2010$ 5:35:10 PM \\
\hline 20000098078 & 6.322 & -1.384 & 0.924 & 11/11/2010 5:35:10 PM \\
\hline 20000100089 & 0.062 & -0.028 & 0.128 & 11/11/2010 5:35:10 PM \\
\hline 20000098170 & -4.575 & -0.996 & 0.179 & 11/11/2010 5:35:10 PM \\
\hline 20000098078 & 6.314 & -1.386 & 0.931 & 11/11/2010 5:35:10 PM \\
\hline 20000100089 & 0.057 & -0.031 & 0.139 & 11/11/2010 5:35:10 PM \\
\hline 20000098169 & -5.554 & -0.753 & 1.512 & 11/11/2010 5:35:10 PM \\
\hline 20000098170 & -4.57 & -0.994 & 0.171 & $11 / 11 / 20105: 35: 10 \mathrm{PM}$ \\
\hline 20000098174 & 11.591 & 0.315 & -0.259 & 11/11/2010 5:35:11 PM \\
\hline 20000098078 & 6.316 & -1.387 & 0.929 & 11/11/2010 5:35:11 PM \\
\hline 20000100089 & -0.276 & -0.041 & 0.668 & 11/11/2010 5:35:11 PM \\
\hline 20000098170 & -4.569 & -0.985 & 0.17 & 11/11/2010 5:35:11 PM \\
\hline 20000098078 & 6.314 & -1.394 & 0.926 & $11 / 11 / 2010$ 5:35:11 PM \\
\hline 20000100089 & -0.25 & -0.041 & 0.634 & 11/11/2010 5:35:11 PM \\
\hline 20000098169 & -5.785 & -0.866 & 1.392 & 11/11/2010 5:35:11 PM \\
\hline 20000098170 & -4.569 & -0.979 & 0.176 & 11/11/2010 5:35:11 PM \\
\hline 20000098174 & 11.631 & 0.343 & -0.248 & 11/11/2010 5:35:11 PM \\
\hline 20000098078 & 6.31 & -1.395 & 0.908 & 11/11/2010 5:35:12 PM \\
\hline 20000100089 & -0.218 & -0.042 & 0.639 & 11/11/2010 5:35:12 PM \\
\hline 20000098170 & -4.566 & -0.98 & 0.175 & 11/11/2010 5:35:12 PM \\
\hline 20000098078 & 6.317 & -1.398 & 0.893 & 11/11/2010 5:35:12 PM \\
\hline 20000100089 & -0.191 & -0.043 & 0.615 & $11 / 11 / 2010$ 5:35:12 PM \\
\hline 20000098169 & -6.61 & -0.615 & 1.563 & $11 / 11 / 2010$ 5:35:12 PM \\
\hline 20000098170 & -4.562 & -0.979 & 0.172 & 11/11/2010 5:35:12 PM \\
\hline 20000098174 & 11.584 & 0.365 & -0.224 & 11/11/2010 5:35:12 PM \\
\hline 20000098078 & 6.324 & -1.407 & 0.872 & 11/11/2010 5:35:12 PM \\
\hline 20000100089 & -0.17 & -0.045 & 0.602 & $11 / 11 / 2010$ 5:35:12 PM \\
\hline 20000098170 & -4.566 & -0.975 & 0.174 & 11/11/2010 5:35:13 PM \\
\hline 20000098078 & 6.321 & -1.413 & 0.882 & $11 / 11 / 2010$ 5:35:13 PM \\
\hline 20000100089 & -0.15 & -0.05 & 0.572 & $11 / 11 / 2010$ 5:35:13 PM \\
\hline 20000098169 & -12.466 & -0.035 & 2.527 & 11/11/2010 5:35:13 PM \\
\hline 20000098170 & -4.564 & -0.976 & 0.17 & 11/11/2010 5:35:13 PM \\
\hline
\end{tabular}




\begin{tabular}{ccccc}
\hline 20000098174 & 11.629 & 0.421 & -0.384 & $11 / 11 / 2010$ 5:35:13 PM \\
20000098078 & 6.315 & -1.393 & 0.917 & $11 / 11 / 20105: 35: 13$ PM \\
20000100089 & -0.129 & -0.051 & 0.544 & $11 / 11 / 20105: 35: 13$ PM \\
20000098170 & -4.563 & -0.977 & 0.163 & $11 / 11 / 20105: 35: 14$ PM \\
20000098078 & 6.31 & -1.391 & 0.963 & $11 / 11 / 20105: 35: 14$ PM \\
20000100089 & -0.111 & -0.048 & 0.512 & $11 / 11 / 20105: 35: 14$ PM \\
20000098169 & -7.179 & -0.622 & 1.146 & $11 / 11 / 20105: 35: 14$ PM \\
20000098170 & -4.575 & -0.983 & 0.156 & $11 / 11 / 20105: 35: 14$ PM \\
20000098174 & 11.583 & 0.33 & -0.191 & $11 / 11 / 20105: 35: 14$ PM \\
20000098078 & 6.313 & -1.392 & 0.983 & $11 / 11 / 20105: 35: 14$ PM \\
20000100089 & -0.097 & -0.045 & 0.475 & $11 / 11 / 20105: 35: 14$ PM \\
20000098170 & -4.583 & -0.984 & 0.158 & $11 / 11 / 20105: 35: 14$ PM \\
20000098078 & 6.315 & -1.379 & 1.028 & $11 / 11 / 20105: 35: 15$ PM \\
20000100089 & -0.088 & -0.047 & 0.439 & $11 / 11 / 20105: 35: 15$ PM \\
20000098169 & -6.847 & -0.723 & 1.276 & $11 / 11 / 20105: 35: 15$ PM \\
20000098170 & -4.583 & -0.988 & 0.151 & $11 / 11 / 20105: 35: 15$ PM \\
20000098174 & 11.638 & 0.346 & -0.269 & $11 / 11 / 20105: 35: 15$ PM \\
20000098078 & 6.319 & -1.379 & 1.049 & $11 / 11 / 20105: 35: 15$ PM \\
20000100089 & -0.072 & -0.046 & 0.401 & $11 / 11 / 20105: 35: 15$ PM \\
20000098170 & -4.581 & -0.99 & 0.139 & $11 / 11 / 20105: 35: 15$ PM \\
20000098078 & 6.323 & -1.383 & 1.052 & $11 / 11 / 20105: 35: 15$ PM \\
20000100089 & -0.066 & -0.045 & 0.361 & $11 / 11 / 20105: 35: 15$ PM \\
20000098169 & -5.649 & -0.865 & 1.53 & $11 / 11 / 20105: 35: 16$ PM \\
20000098170 & -4.582 & -0.99 & 0.128 & $11 / 11 / 20105: 35: 16$ PM \\
20000098174 & 11.628 & 0.346 & -0.288 & $11 / 11 / 20105: 35: 16$ PM \\
20000098078 & 6.327 & -1.389 & 1.04 & $11 / 11 / 20105: 35: 16$ PM \\
20000100089 & -0.053 & -0.044 & 0.331 & $11 / 11 / 20105: 35: 16$ PM \\
\hline
\end{tabular}


Table C-3: True Coordinates of Fixed Points Captured Using Total Station Survey Equipment

\begin{tabular}{lcccccc}
\hline & \multicolumn{3}{c}{ Total Station Coordinates } & \multicolumn{3}{c}{ Global True Coordinates } \\
\hline ID & $\mathrm{X}$ & $\mathrm{V}$ & $\mathrm{Z}$ & $\mathrm{X}$ & $\mathrm{y}$ & $\mathrm{Z}$ \\
line1_1 & 1003.952 & 998.351 & 101.607 & -3.952 & -1.649 & 1.607 \\
line1_2 & 997.258 & 1001.707 & 101.605 & 2.742 & 1.707 & 1.605 \\
line1_3 & 1000.000 & 1000.000 & 100.000 & 0.000 & 0.000 & 0.000 \\
line1_4 & 991.778 & 1000.045 & 100.003 & 8.222 & 0.045 & 0.003 \\
line1_5 & 988.645 & 1000.001 & 99.994 & 11.356 & 0.001 & -0.006 \\
line1_6 & 1003.497 & 999.318 & 101.185 & -3.497 & -0.682 & 1.185 \\
line2_1 & 1004.641 & 1000.045 & 100.001 & -4.641 & 0.045 & 0.001 \\
line2_2 & 1000.605 & 999.485 & 101.076 & -0.605 & -0.515 & 1.076 \\
line2_3 & 992.082 & 999.312 & 101.665 & 7.918 & -0.688 & 1.665 \\
line2_4 & 1003.471 & 1000.698 & 101.269 & -3.471 & 0.698 & 1.269 \\
line2_5 & 1003.495 & 999.320 & 101.716 & -3.495 & -0.680 & 1.716 \\
line3_1 & 1005.037 & 1000.017 & 100.003 & -5.037 & 0.016 & 0.003 \\
line3_2 & 993.876 & 1000.585 & 101.081 & 6.124 & 0.585 & 1.081 \\
line3_3 & 992.093 & 999.298 & 102.255 & 7.908 & -0.702 & 2.255 \\
line3_4 & 1004.478 & 1000.730 & 101.186 & -4.478 & 0.730 & 1.186 \\
line3_5 & 1003.498 & 999.319 & 102.255 & -3.498 & -0.681 & 2.255 \\
line3_6 & 1003.457 & 1000.696 & 101.860 & -3.457 & 0.696 & 1.860 \\
line4_1 & 993.252 & 1000.647 & 101.827 & 6.748 & 0.647 & 1.827 \\
line4_2 & 993.696 & 999.453 & 101.076 & 6.304 & -0.547 & 1.076 \\
line4_3 & 993.230 & 1000.649 & 101.225 & 6.770 & 0.649 & 1.225 \\
line4_4 & 992.091 & 999.325 & 101.181 & 7.909 & -0.676 & 1.181 \\
line4_5 & 993.552 & 1000.669 & 101.372 & 6.448 & 0.669 & 1.372 \\
line4_6 & 1004.455 & 1000.734 & 101.806 & -4.455 & 0.734 & 1.806 \\
\hline & & & & & & \\
\hline
\end{tabular}

Table C-4: Input Data for Four Lines for Installation Activity on October $30^{\text {th }} \mathbf{2 0 1 0}$

\begin{tabular}{ccccc}
\hline Spool & $\begin{array}{c}\text { UWB Error } \\
(\mathbf{m})\end{array}$ & $\begin{array}{c}\text { Laser } \\
\text { Scanning }\end{array}$ & $\begin{array}{c}\text { Percent } \\
\text { Contribution }\end{array}$ & Length $(\mathbf{m})$ \\
\hline Spool1_1 & 0.15 & 1 & $10 \%$ & 3.6 \\
Spool1_2 & - & 1 & $10 \%$ & 3.6 \\
Spool1_3 & - & 1 & $4 \%$ & 1.6 \\
Spool1_4 & - & 0 & $4 \%$ & 1.6 \\
Spool1_5 & 0.24 & 0 & $11 \%$ & 3.9 \\
Spool1_6 & - & 0 & $11 \%$ & 3.9 \\
Spool1_7 & - & 0 & $4 \%$ & 1.4 \\
\hline
\end{tabular}




\begin{tabular}{lcccc}
\hline Spool1_8 & - & 0 & $4 \%$ & 1.4 \\
Spool1_9 & - & 0 & $21 \%$ & 7.4 \\
Spool1_10 & - & 0 & $21 \%$ & 7.4 \\
Spool2_1 & 0.21 & 1 & $8 \%$ & 2.0 \\
Spool2_2 & - & 1 & $8 \%$ & 2.0 \\
Spool2_3 & - & 1 & $17 \%$ & 4.0 \\
Spool2_4 & 0.13 & 1 & $17 \%$ & 4.0 \\
Spool2_5 & - & 0 & $23 \%$ & 5.5 \\
Spool2_6 & - & 0 & $23 \%$ & 5.5 \\
Spool2_7 & - & 0 & $2 \%$ & 0.6 \\
Spool2_8 & 0.11 & 0 & $2 \%$ & 0.6 \\
Spool3_1 & 0.22 & 0 & $20 \%$ & 5.4 \\
Spool3_2 & - & 0 & $20 \%$ & 5.4 \\
Spool3_3 & - & 0 & $3 \%$ & 0.9 \\
Spool3_4 & - & 0 & $3 \%$ & 0.9 \\
Spool3_5 & 0.2 & 0 & $22 \%$ & 5.9 \\
Spool3_6 & - & 0 & $22 \%$ & 5.9 \\
Spool3_7 & - & 0 & $2 \%$ & 0.6 \\
Spool3_8 & - & 0 & $2 \%$ & 0.6 \\
Spool3_9 & - & 0 & $2 \%$ & 0.5 \\
Spool3_10 & - & 0 & $2 \%$ & 0.5 \\
Spool4_1 & 0.33 & 1 & $4 \%$ & 0.5 \\
Spool4_2 & - & 1 & $4 \%$ & 0.5 \\
Spool4_3 & - & 1 & $1 \%$ & 0.2 \\
Spool4_4 & - & 1 & $1 \%$ & 0.2 \\
Spool4_5 & 0.25 & 1 & $3 \%$ & 0.4 \\
Spool4_6 & - & 0 & $3 \%$ & 0.4 \\
Spool4_7 & - & 0 & $4 \%$ & 0.5 \\
Spool4_8 & - & 0 & $4 \%$ & 0.5 \\
Spool4_9 & - & 0 & $38 \%$ & 5.2 \\
Spool4_10 & - & 0 & $38 \%$ & 5.2 \\
\hline & & & & \\
\hline
\end{tabular}


Table C-5: UWB Generated Paths for Activity Progress Tracking for Four Lines on October $30^{\text {th }} 2010$

\begin{tabular}{cccc}
\hline Line & Activity & Path Length & Start Spool \\
\hline Line 1 & Installation & 11.98 & Spool1_3 \\
& Welding & 8.90 & Spool1_1 \\
& Inspection & 6.97 & Spool1_1 \\
Line 2 & Installation & 12.92 & Spool2_5 \\
& Welding & 11.76 & Spool2_1 \\
& Inspection & - & - \\
Line 3 & Installation & 15.22 & Spool3_5 \\
& Welding & 14.10 & Spool3_1 \\
& Inspection & - & - \\
Line 4 & Installation & 7.14 & Spool4_1 \\
& Welding & - & - \\
& Inspection & - & - \\
\hline
\end{tabular}

Table C-6: Fusion Results for Installation Activity for Four Lines for Progress on October

\begin{tabular}{cccc} 
Spol & $\mathbf{3 0 1 0}$ \\
\hline Spool & $\begin{array}{c}\text { Object Recognition } \\
\text { from Laser Scanning }\end{array}$ & $\begin{array}{c}\text { Materials tracking } \\
\text { fusion }\end{array}$ & Activity Based fusion \\
\hline Spool1_1 & 1 & 1 & 1 \\
Spool1_2 & 1 & 1 & 1 \\
Spool1_3 & 1 & 1 & 1 \\
Spool1_4 & 0 & 1 & 1 \\
Spool1_5 & 0 & 1 & 1 \\
Spool1_6 & 0 & 0 & 1 \\
Spool1_7 & 0 & 0 & 0 \\
Spool1_8 & 0 & 0 & 0 \\
Spool1_9 & 0 & 0 & 0 \\
Spool1_10 & 0 & 0 & 0 \\
Spool2_1 & 1 & 1 & 1 \\
Spool2_2 & 1 & 1 & 1 \\
Spool2_3 & 1 & 1 & 1 \\
Spool2_4 & 1 & 1 & 1 \\
Spool2_5 & 0 & 1 & 1 \\
Spool2_6 & 0 & 1 & 1 \\
Spool2_7 & 0 & 1 & \\
\hline
\end{tabular}




\begin{tabular}{llll}
\hline Spool2_8 & 0 & 1 & 1 \\
Spool3_1 & 0 & 1 & 1 \\
Spool3_2 & 0 & 1 & 1 \\
Spool3_3 & 0 & 1 & 1 \\
Spool3_4 & 0 & 1 & 1 \\
Spool3_5 & 0 & 1 & 1 \\
Spool3_6 & 0 & 0 & 1 \\
Spool3_7 & 0 & 0 & 1 \\
Spool3_8 & 0 & 0 & 1 \\
Spool3_9 & 0 & 0 & 1 \\
Spool3_10 & 0 & 0 & 1 \\
Spool4_1 & 1 & 1 & 1 \\
Spool4_2 & 1 & 1 & 1 \\
Spool4_3 & 1 & 1 & 1 \\
Spool4_4 & 1 & 1 & 1 \\
Spool4_5 & 1 & 1 & 1 \\
Spool4_6 & 0 & 0 & 1 \\
Spool4_7 & 0 & 0 & 1 \\
Spool4_8 & 0 & 0 & 1 \\
Spool4_9 & 0 & 0 & 0 \\
Spool4_10 & 0 & 0 & 0 \\
\hline
\end{tabular}

Table C-7: Intermediary Results for Progress of Four Lines on October $30^{\text {th }} 2010$

\begin{tabular}{ccccccc}
\hline Spool & $\begin{array}{c}\text { True } \\
\text { Progress } \\
\text { Installation }\end{array}$ & $\begin{array}{c}\text { Estimated } \\
\text { Progress } \\
\text { Installation }\end{array}$ & $\begin{array}{c}\text { True } \\
\text { Progress } \\
\text { Welding }\end{array}$ & $\begin{array}{c}\text { Estimated } \\
\text { Progress } \\
\text { Welding }\end{array}$ & $\begin{array}{c}\text { True } \\
\text { Progress } \\
\text { Inspection }\end{array}$ & $\begin{array}{c}\text { Estimated } \\
\text { Progress } \\
\text { Inspection }\end{array}$ \\
\hline Spool1_1 & 1 & 1 & 1 & 1 & 1 & 1 \\
Spool1_2 & 1 & 1 & 1 & 1 & 1 & 1 \\
Spool1_3 & 1 & 1 & 1 & 1 & 0 & 0 \\
Spool1_4 & 1 & 1 & 0 & 0 & 0 & 0 \\
Spool1_5 & 1 & 1 & 0 & 0 & 0 & 0 \\
Spool1_6 & 1 & 1 & 0 & 0 & 0 & 0 \\
Spool1_7 & 0 & 0 & 0 & 0 & 0 & 0 \\
Spool1_8 & 0 & 0 & 0 & 0 & 0 & 0 \\
Spool1_9 & 0 & 0 & 0 & 0 & 0 & 0 \\
Spool1_10 & 0 & 0 & 0 & 0 & 0 & 0 \\
Spool2_1 & 1 & 1 & 1 & 1 & - & - \\
Spool2_2 & 1 & 1 & 1 & 1 & - & - \\
\hline
\end{tabular}




\begin{tabular}{lllllll}
\hline Spool2_3 & 1 & 1 & 1 & 1 & - & - \\
Spool2_4 & 1 & 1 & 1 & 1 & - & - \\
Spool2_5 & 1 & 1 & 0 & 0 & - & - \\
Spool2_6 & 1 & 1 & 0 & 0 & - & - \\
Spool2_7 & 1 & 1 & 0 & 0 & - & - \\
Spool2_8 & 1 & 1 & 0 & 0 & - & - \\
Spool3_1 & 1 & 1 & 1 & 1 & - & - \\
Spool3_2 & 1 & 1 & 1 & 1 & - & - \\
Spool3_3 & 1 & 1 & 1 & 1 & - & - \\
Spool3_4 & 1 & 1 & 1 & 1 & - & - \\
Spool3_5 & 1 & 1 & 0 & 0 & - & - \\
Spool3_6 & 1 & 1 & 0 & 0 & - & - \\
Spool3_7 & 1 & 1 & 0 & 0 & - & - \\
Spool3_8 & 1 & 1 & 0 & 0 & - & - \\
Spool3_9 & 1 & 1 & 0 & 0 & - & - \\
Spool3_10 & 1 & 1 & 0 & 0 & - & - \\
Spool4_1 & 1 & 1 & - & - & - & - \\
Spool4_2 & 1 & 1 & - & - & - & - \\
Spool4_3 & 1 & 1 & - & - & - \\
Spool4_4 & 1 & 1 & - & - & - \\
Spool4_5 & 1 & 1 & - & - & - & - \\
Spool4_6 & 1 & 1 & - & - & - & - \\
Spool4_7 & 1 & 1 & - & - & - \\
Spool4_8 & 1 & 1 & - & - & - & - \\
Spool4_9 & 0 & 0 & - & - & - \\
Spool4_10 & 0 & 0 & - & - & - & - \\
\hline & & 1 & & - & - \\
\hline
\end{tabular}


Table C-8: Final Activity Progress Estimates for October 30 2010

\begin{tabular}{ccc}
\hline Line & Activity & Estimated progress \\
\hline Line 1 & Installation & $51 \%$ \\
& Welding & $30 \%$ \\
& Inspection & $20 \%$ \\
& Delivery & $67 \%$ \\
Line 2 & Total Line Progress & $51 \%$ \\
& Installation & $100 \%$ \\
& Welding & $50 \%$ \\
& Inspection & $0 \%$ \\
Line 3 & Delivery & $100 \%$ \\
& Total Line Progress & $85 \%$ \\
& Installation & $100 \%$ \\
& Welding & $40 \%$ \\
& Inspection & $0 \%$ \\
Line 4 & Delivery & $100 \%$ \\
& Total Line Progress & $83 \%$ \\
& Installation & $24 \%$ \\
& Welding & $0 \%$ \\
& Inspection & $0 \%$ \\
& Delivery & $67 \%$ \\
& Total Line Progress & $33 \%$ \\
\hline
\end{tabular}




\section{Appendix D}

\section{Terminology}

$\begin{array}{ll}\text { AEC\&FM } & \text { Architectural, Engineering, Construction and Facility Management } \\ \text { BIM } & \text { Building Information Model } \\ \text { DMS } & \text { Data Management System }\end{array}$

EPPMS Electronic Product and Process Management System

GPS Global Positioning System

JDL Joint Directors of Laboratories

LADAR Laser Detection and Ranging

NIH National Institute of Health

NIJ National Institute of Justice

NSF National Science Foundation

RFID Radio Frequency Identification

Spool Any individual pipe section that would show up as a separate entity in the 3D CAD or BIM Model

UWB Ultra Wide Band

WfMC Workflow Management Coalition

Workflow A term used to describe, execute and control the sequence of tasks in a business process, including procedural steps, people or stakeholders involved, as well as the input and output that is required 